\title{
ASYMPTOTIC TORSION AND TOEPLITZ OPERATORS
}

\author{
JEAN-MICHEL BISMUT ${ }^{1}$, XIAONAN MA ${ }^{2}$ AND WEIPING ZHANG ${ }^{3}$ \\ ${ }^{1}$ Département de Mathématique, Université Paris-Sud, Bâtiment 425, \\ F-91405 Orsay, France (Jean-Michel.Bismut@math.u-psud.fr) \\ ${ }^{2}$ Université Paris 7, UFR de Mathématiques, Case 7012, \\ F-75205 Paris Cedex 13, France (xiaonan.ma@imj-prg.fr) \\ ${ }^{3}$ Chern Institute of Mathematics \& LPMC, Nankai University, \\ Tianjin 300071, PR China (weiping@nankai.edu.cn)
}

(Received 29 June 2014; accepted 12 April 2015; first published online 11 June 2015) This paper is dedicated to the memory of Professor Louis Boutet de Monvel

\begin{abstract}
We use Toeplitz operators to evaluate the leading term in the asymptotics of the analytic torsion forms associated with a family of flat vector bundles $F_{p}$. For $p \in \mathbf{N}$, the flat vector bundle $F_{p}$ is the direct image of $L^{p}$, where $L$ is a holomorphic positive line bundle on the fibres of a flat fibration by compact Kähler manifolds. The leading term of the analytic torsion forms is the integral along the fibre of a locally defined differential form.
\end{abstract}

Keywords: index theory and related fixed point theorems; determinants and determinant bundles; analytic torsion

2010 Mathematics subject classification: Primary 58J20; 58J52

\section{Contents}

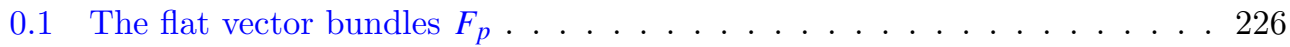

0.2 A nondegeneracy condition . . . . . . . . . . . . . . . . . . . . . . . . . . . . . . . . . . . 227

$0.3 V$-invariant and $W$-invariant . . . . . . . . . . . . . . . 227

0.4 Nondegeneracy, Toeplitz operators, and the spectral gap . . . . . . . . . . 229

0.5 Analytic torsion, analytic torsion forms, and $\Gamma$-torsion . . . . . . . . . . . 229

0.6 Asymptotic torsion and the trace formula . . . . . . . . . . . . . . . 229

0.7 The main result . . . . . . . . . . . . . . . . . . . . . . . . . . . . . . . . . . . . . . .

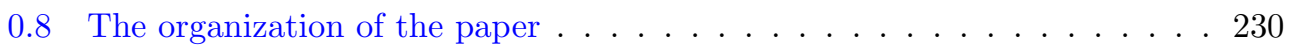

J.-M. Bismut is indebted to W. Müller for exciting discussions on the content of this paper. J.-M. Bismut has received funding from the European Research Council (E.R.C.) under the European Union's Seventh Framework Program (FP7/2007-2013)/ERC grant agreement no. 291060.

X. Ma thanks Institut Universitaire de France (I.U.F.) for its support.

W. Zhang was partially supported by MOEC and NNSFC. 
1 Multidimensional transgressions of the Euler form $\quad 231$

1.1 Reductive groups . . . . . . . . . . . . . . . . . . . 231

1.2 Clifford algebras . . . . . . . . . . . . . . . . . . . 232

1.3 The algebra $\widehat{c}(\widehat{E}) \otimes U \mathfrak{g} \ldots \ldots \ldots \ldots \ldots \ldots$. . . . . . . . . . . . . . . . . . . . . . . . . . . . . . . .

1.4 A complex $G$-manifold and the function $R \ldots \ldots \ldots$. . . . . . 234

1.5 The Berezin integral . . . . . . . . . . . . . . . . . . . . . . . . . . . . . . . . . . . . . . . . . . .

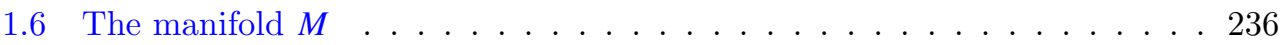

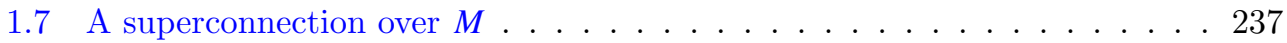

1.8 The principal symbol as a rescaled limit . . . . . . . . . . . . . 239

1.9 The Bianchi identity . . . . . . . . . . . . . . . . . . . . . . . . . . . . . . . . . . . . . . . . . .

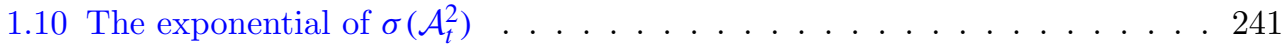

1.11 The Mathai-Quillen forms $\underline{a}_{t}, \underline{b}_{t} \ldots \ldots \ldots \ldots$. . . . . . . . . . . . . . . . . . . . . . . .

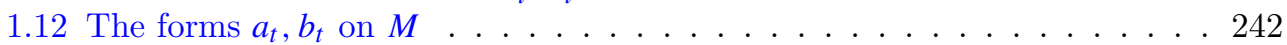

2 The $W$-invariant $\quad \mathbf{2 4 6}$

2.1 A smooth fibration . . . . . . . . . . . . . . . . . 246

2.2 A metric on $T X$ and the tensors $T$ and $S \ldots \ldots \ldots \ldots$

2.3 The case where $\widehat{E}=\widehat{T X}, \beta=\widehat{\theta}^{\mathfrak{p}} \ldots \ldots \ldots \ldots \ldots . \ldots \ldots$

2.4 Exchanging the hatted and unhatted variables . . . . . . . . . . . 249

2.5 The forms $c_{t}, d_{t}$ on $M \ldots \ldots \ldots \ldots \ldots \ldots$. . . . . . . . . . . . . . . . . . . . . . . . . . . . . . . . .

2.6 The $W$-invariant $\ldots \ldots \ldots \ldots \ldots \ldots \ldots \ldots \ldots$

2.7 The forms $\pi_{*} W$ and adiabatic limits . . . . . . . . . . . 256

2.8 Rigidity . . . . . . . . . . . . . . . . . . . . 259

3 A class of Toeplitz operators $\quad \mathbf{2 6 0}$

3.1 A line bundle on $N \ldots \ldots \ldots \ldots \ldots$. . . . . . . . . . . . . . . . . . . . . . . . .

3.2 Lie derivatives and Toeplitz operators . . . . . . . . . . . . . . . . . . . . . . . . . . . . . . . . . 262

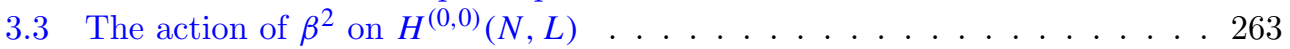

3.4 The asymptotics of the trace of $e^{A / p} \ldots \ldots \ldots \ldots$

3.5 A heat operator acting on $\chi_{p} \ldots \ldots \ldots$. . . . . . . . . . . . . . . . . . . . .

4 A lower bound for the Hodge Laplacian $\quad \mathbf{2 6 6}$

4.1 A flat vector bundle . . . . . . . . . . . . . . . . . 266

4.2 A formula for the Hodge Laplacian . . . . . . . . . . . . . . . . . . . . . . . . . . . . . . . . . . . . . .

4.3 Nondegeneracy of $\widehat{\theta}^{\mathfrak{p}}$ and gap in the spectrum . . . . . . . . . . . 268

5 The analytic torsion forms $\quad \mathbf{2 6 9}$

5.1 The odd forms associated with a flat vector bundle . . . . . . . . . 270

5.2 A flat superconnection on $\Omega\left(X,\left.F\right|_{X}\right) \ldots \ldots \ldots \ldots \ldots$

5.3 The adjoint superconnection . . . . . . . . . . . . . . . . 271

5.4 A rescaling of the metric on $T X \ldots \ldots \ldots \ldots \ldots 272 \ldots \ldots \ldots \ldots$

5.5 The odd closed forms $h\left(A^{\prime}, g_{t}^{\Omega\left(X,\left.F\right|_{X}\right)}\right) \ldots \ldots \ldots \ldots \ldots$

5.6 A transgression formula . . . . . . . . . . . . . . . . . 274

5.7 The analytic torsion forms . . . . . . . . . . . . . . . . . . . . . . . . . . . . . . . . . . . . . . .

5.8 The odd forms associated with $H^{(0,0)}\left(N, L^{p}\right) \ldots \ldots \ldots . \ldots . \ldots 277$

5.9 A Lichnerowicz formula . . . . . . . . . . . . . . . . . . . 277 
6 The asymptotics of the odd superconnection forms $\quad \mathbf{2 7 8}$

6.1 The asymptotics of the forms $h\left(\nabla^{F_{p}}, g^{F_{p}}\right) \ldots \ldots \ldots . \ldots . \ldots 279$

6.2 The universal construction of the operators $C_{t}, D_{t} \ldots \ldots \ldots$

6.3 Lifting $L_{t}^{\mathscr{F}} \ldots \ldots \ldots \ldots \ldots \ldots \ldots \ldots$

6.4 A formula for $h_{t}\left(A^{\prime}, g_{t}^{\Omega\left(X,\left.F\right|_{X}\right)}\right) \ldots \ldots \ldots \ldots \ldots . \ldots \ldots 28 \ldots \ldots$

6.5 The asymptotics as $p \rightarrow+\infty$ of the odd superconnection forms . . . . . 283

6.6 Convergence results on a Galois cover of $X \ldots \ldots$. . . . . . . . . . . . . . 290

7 The asymptotics of the analytic torsion forms $\quad 291$

7.1 The main result . . . . . . . . . . . . . . . . . . 291

7.2 A proof of the first equation in $(7.4) \ldots \ldots \ldots . \ldots . \ldots . \ldots 292$

7.3 A proof of the second equation in $(7.4) \ldots \ldots . \ldots . \ldots 293$

7.4 Compatibility of Theorem 7.1 to known results . . . . . . . . . . . . . . 297

7.5 Rigidity of the class of $\pi_{*} W \ldots \ldots \ldots \ldots$. . . . . . . . . . . . . . . . . . . . . . . . . . . . . . . . .

7.6 The asymptotics of the $\Gamma$-torsion . . . . . . . . . . . . . 298

8 Asymptotic torsion and orbital integrals $\quad 299$

8.1 Reductive groups and symmetric spaces . . . . . . . . . . . . . . . . . . . . . 300

8.2 The Casimir operator of $G \ldots \ldots$. . . . . . . . . . . . . . . . . . . . . . . . . . . . . . . . . . .

8.3 The complexification of compact Lie groups . . . . . . . . . . . . . . . . . . . . . . . . 302

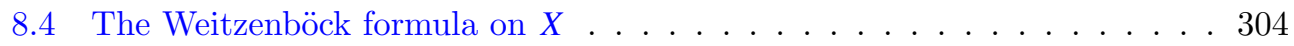

8.5 The trivial orbital integrals . . . . . . . . . . . . . . . . . . . . . . . . . . . . . . . . . . . . . . .

8.6 The vanishing of the forms $d_{t} \ldots \ldots \ldots$. . . . . . . . . . . . . . . . . . . . . . . . . . . . . . . . . . .

8.7 The $W$-invariant for $\mathrm{SL}_{2}(\mathbf{C}) \ldots \ldots \ldots \ldots$

8.8 The nondegenerate representations of $U \ldots \ldots$. . . . . . . . . . . . . . . . . . . . . . . . . . . . .

8.9 The asymptotics as $p \rightarrow+\infty$ of the orbital integrals . . . . . . . . . 316

9 The case of general Lie groups $\quad 319$

9.1 The case of a general Lie group $G \ldots \ldots$. . . . . . . . . . . . 321

9.2 Metric properties of the projection $q \ldots \ldots . \ldots \ldots 22$

9.3 The bundle of Poisson algebras . . . . . . . . . . . . . . . . . . . . . . . . . . . . . . . . . . . . . . . . .

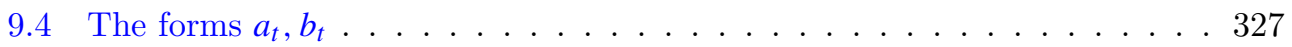

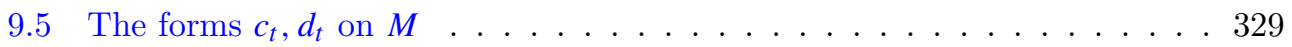

9.6 The $W$-invariant . . . . . . . . . . . . . . . . . . . . 332

9.7 The curvature of the unitary connection on $F \ldots \ldots$. . . . . . . . . . 333

9.8 The algebra of Toeplitz operators . . . . . . . . . . . . . . . . . . . . . . . . . . . . . . . . . .

9.9 The asymptotics of the forms $h\left(\nabla^{F_{p}}, g^{F_{p}}\right) \ldots \ldots \ldots 33$

9.10 The spectral gap . . . . . . . . . . . . . . . . . . . . . . . . . . . . . . . . . . . . . . . . . . . . . .

9.11 A formula for $L_{t}^{F} \ldots \ldots \ldots 37$

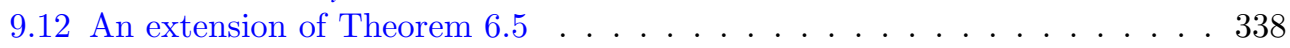

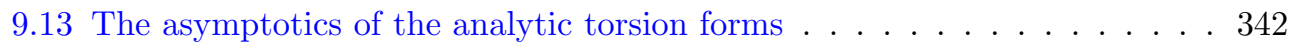

9.14 The case of reductive groups . . . . . . . . . . . . . . 343

$\begin{array}{ll}\text { Index } & 343\end{array}$

$\begin{array}{lr}\text { References } & \mathbf{3 4 7}\end{array}$ 


\section{Introduction}

Real analytic torsion is a spectral invariant of a compact Riemannian manifold equipped with a flat Hermitian vector bundle; it was introduced by Ray and Singer [44]. For odd-dimensional manifolds, this invariant does not depend on the metric data. Ray and Singer conjectured that, for unitarily flat vector bundles, this invariant coincides with the Reidemeister torsion, a topological invariant. This conjecture was established by Cheeger [28] and Müller [41], and extended by Müller [42] for unimodular flat vector bundles, and by Bismut and Zhang [22] to arbitrary flat vector bundles. A fundamental property of these invariants is that they are well defined for manifolds that are not oriented.

The purpose of this paper is to use Toeplitz operators to study asymptotic properties of the Ray-Singer real analytic torsion [44]. More specifically, if $X$ is a compact Riemannian manifold, and if $\left.F_{p}\right|_{p \in \mathbf{N}}$ is a certain family of flat Hermitian vector bundles on $X$, we obtain the asymptotics of their Ray-Singer torsion as $p \rightarrow+\infty$. We express the leading term of the asymptotic torsion as the integral of a differential form that is obtained by a universal construction that uses the Berezin integral formalism introduced by Mathai and Quillen [39]. For compact odd-dimensional manifolds, we obtain in this way an infinite number of invariants that are computable using local data.

Let us now give the background to the results obtained in this paper. For holomorphic torsion, this study was initiated by Bismut and Vasserot [20, 21]. For real analytic torsion, Bergeron and Venkatesh [3] have studied the asymptotics of the analytic torsion of quotients of symmetric spaces by a decreasing sequence of lattices in the underlying Lie group. Their motivation was to understand the behaviour of the torsion subgroup in the cohomology of compact quotients. Müller [43] studied in detail the case where $X$ is a compact quotient of the three-dimensional hyperbolic space $\mathrm{SL}_{2}(\mathbf{C}) / \mathrm{SU}(2)$, and $F_{p}=S^{p} F$, where $F$ is the two-dimensional flat vector bundle associated with the tautological representation of $\mathrm{SL}_{2}(\mathbf{C})$ on $\mathbf{C}^{2}$, and $S^{p}$ denotes the $p$ th symmetric power. The arguments of [43] are based on Selberg's trace formula. Marshall and Müller [38] have used the results of [43] to study the asymptotics of torsion subgroups in the cohomology of hyperbolic 3-manifolds.

Our approach to the asymptotics of Ray-Singer torsion for the vector bundles $F_{p}$ is valid for an arbitrary compact manifold $X$. Moreover, we work in a more general context than the one above, since we also obtain the asymptotics as $p \rightarrow+\infty$ of the analytic torsion forms of Bismut and Lott [18]. In that context, let $\pi: M \rightarrow S$ be a submersion of smooth manifolds with compact fibre $X$. Our flat bundles $F_{p}$ are obtained as direct images by a proper map $q: \mathcal{N} \rightarrow M$ of the $p$ th power of a holomorphic positive line bundle $L$ along the compact Kähler fibre $N$, under a nondegeneracy assumption that is ultimately related to geometric quantization, and to the corresponding Toeplitz operators in the sense of $[23,25,34]$.

Let us now give more details on the main points that are covered in the paper.

\subsection{The flat vector bundles $F_{p}$}

In the paper, we work under two kinds of assumption. In the first eight sections of the paper, we assume that $G$ is a reductive Lie group acting holomorphically on the 
manifold $N$, and that $P_{G} \rightarrow M$ is a flat $G$-principal bundle on $M$, so that $F_{p}$ is the flat vector bundle on $M$ that is obtained via the action of $G$ on $H^{(0,0)}\left(N, L^{p}\right)$. Then we can transfer the analysis of the asymptotic torsion to the fibre bundle $P_{G}$, which does not depend on $p$. The results are ultimately formulated in terms of generalized characteristic forms on $M$, which are expressed in terms of differential operators acting on the Lie algebra $\mathfrak{g}$ of $G$.

In $\S 9$, we consider the case where $G$ is an arbitrary Lie group. In this more general case, the answer is expressed as the integral along the fibre $N$ of an explicit differential form on $\mathcal{N}$. This differential form is constructed using deformations of the Poisson algebra along the fibre $N$. Some key properties of the algebra of Toeplitz operators are used to obtain the final formula.

\subsection{A nondegeneracy condition}

In both cases, our main result applies to the family of flat Hermitian vector bundles $\left.F_{p}\right|_{p \in \mathbf{N}}$ under a natural nondegeneracy condition that guarantees that, for $p \in \mathbf{N}$ large enough, $H^{\cdot}\left(X, F_{p}\right)=0$.

Let us give more details when $G$ is a reductive group with Lie algebra $\mathfrak{g}$. Let $K$ be a maximal compact subgroup with Lie algebra $\mathfrak{k}$. Let $\mathfrak{g}=\mathfrak{p} \oplus \mathfrak{k}$ be the Cartan decomposition of $\mathfrak{g}$. Let $U$ be the compact form of $G$, with Lie algebra $\mathfrak{u}=i \mathfrak{p} \oplus \mathfrak{k}$. We assume that $U$ acts holomorphically on $N$, and that its action lifts to $L$. Let $\mu: N \rightarrow \mathfrak{u}^{*}$ denote the corresponding moment map.

Let $P_{K}$ be a reduction of $P_{G}$ to the subgroup $K$. Let $\theta^{\mathfrak{g}}$ be the connection form on the flat bundle $P_{G}$, and let $\theta^{\mathfrak{k}}$ be the connection form on $P_{K}$ that is induced by $\theta^{\mathfrak{g}}$ via the Cartan decomposition $\mathfrak{g}=\mathfrak{p} \oplus \mathfrak{k}$. Let $\theta^{\mathfrak{p}}$ be the $\mathfrak{p}$ component of $\theta^{\mathfrak{g}}$ on $P_{K}$. Then $\left\langle\mu, i \theta^{\mathfrak{p}}\right\rangle$ is a well-defined section of $q^{*} T^{*} X$. Our nondegeneracy assumption says that this section does not vanish on $\mathcal{N}$.

When $\left(F, \nabla^{F}, g^{F}\right)$ is a flat vector bundle on $M$, an important special case is when $N$ is the projective bundle $\mathbf{P} F$ and $L$ is the canonical hyperplane line bundle on $N$. In this case, $F_{p}=S^{p} F^{*}$. Let $\omega\left(\nabla^{F}, g^{F}\right)$ be the variation of $g^{F}$ with respect to the flat connection $\nabla^{F}$, as defined in (5.1). Our nondegeneracy condition says that, if $z \in \mathbf{P} F$, the section $\left\langle\omega\left(\nabla^{F}, g^{F}\right) z, \bar{z}\right\rangle /|z|^{2}$ of $q^{*} T^{*} X$ should not vanish.

In the general case, let $g^{L}$ be a Hermitian metric on the line bundle $L$ on $\mathcal{N}$, and let $\widehat{T X}$ be another copy of $T X$. The nondegeneracy condition is equivalent to the fact that a section $\sigma_{\widehat{\vartheta}}$ of $q^{*} \widehat{T^{*} X}$ does not vanish on $\mathcal{N}$. The section $\sigma_{\widehat{\vartheta}}$ is obtained by varying the metric of $L$ with respect to the flat connection of $P_{G}$.

\section{3. $V$-invariant and $W$-invariant}

Our main result states that the asymptotics of the analytic torsion can be evaluated by integration on $X$ of a locally computable differential form $W$. Since analytic torsion verifies natural functorial properties with respect to the composition of submersions, the integral of $W$ should verify similar properties.

In $[16, \S 3]$, Bismut and Goette considered a related problem. Namely they gave a local formula for the difference of two natural versions of equivariant analytic torsion. 
The corresponding local object, the $V$-invariant, which is naturally associated with a Killing vector field on $X$, was shown directly to verify the same functorial properties as analytic torsion. Its construction relies on the Berezin integral formalism of Mathai and Quillen [39]. Another copy $\Lambda^{*}\left(\widehat{T^{*} X}\right)$ of $\Lambda^{*}\left(T^{*} X\right)$ is used in the construction. The natural involution exchanging $\Lambda^{*}\left(T^{*} X\right)$ and $\Lambda^{*}\left(\widehat{T^{*} X}\right)$ plays a key role in explaining the properties of the $V$-invariant. The intuition given in [16] for the construction of the $V$-invariant is that analytic torsion itself can be viewed formally as the $V$-invariant of the loop space $L X$ of $X$ equipped with its canonical action of $S^{1}$ by rotations, so that any of its 'localizations' has to follow the same algebraic pattern.

The same algebraic formalism reappears here. Our $W$-invariant is a close analogue of the $V$-invariant, which, instead of being associated with a Killing vector field, is now obtained via the local variation of a nonflat Hermitian metric. The underlying paradigm is that the analytic torsion of a flat vector bundle can be viewed formally as the pairing of the $V$-invariant of $L X$ and the $W$-invariant of $L X$. In the context of [16], only the $V$ part contributes to the localization. Here it is only the $W$ part that contributes to the large- $p$ asymptotics.

Our construction of the $W$-invariant in $\S \S 2$ and 9 is self-contained; it does not necessitate any knowledge of the $V$-invariant of [16], and the above considerations on the loop space will remain in the shadow. The construction is only possible under the assumption of nondegeneracy. In the case where $G$ is reductive, the $W$-invariant, defined in Definition 2.11, is expressed in terms of the action of the heat kernels of certain operators with constant coefficients on $\mathfrak{g}_{\mathbf{C}}$ acting on the Duistermaat-Heckman integral $[29,30]$ on $N$. For a general Lie group, the $W$-invariant is obtained in Definition 9.22 as the integral of a differential form on $\mathcal{N}$. Of course, we show that the two constructions are compatible.

Let $\vartheta$ be the 1 -form on $\mathcal{N}$ which is minus half of the horizontal variation of the metric $g^{L}$ with respect to the flat connection, so that $\sigma_{\widehat{\vartheta}}$ is the restriction of $\vartheta$ to $q^{*} \widehat{T X}$. As we saw before, the nondegeneracy assumption asserts that the section $\sigma_{\widehat{\vartheta}}$ does not vanish. If $m=\operatorname{dim} X$, let $\psi$ be the $(m-1)$-form on the total space of $\widehat{T X} \backslash\{0\}$ that transgresses the Euler form $e\left(T X, \nabla^{T X}\right)$. Let $c_{1}\left(L, g^{L}\right)$ be a natural first Chern form for $\left(L, g^{L}\right)$. In Theorems 2.12 and 9.23 , we establish the explicit formula

$$
W=q_{*}\left[\vartheta \sigma_{\widehat{\vartheta}}^{*} \psi \exp \left(c_{1}\left(L, g^{L}\right)\right)\right] .
$$

A very special case is when $N$ is reduced to a point, so that $\left(L, \nabla^{L}\right)$ is a flat line bundle on $M$. If $\omega\left(\nabla^{L}, g^{L}\right)=\left(g^{L}\right)^{-1} \nabla^{L} g^{L}$, then $\vartheta=-\omega\left(\nabla^{L}, g^{L}\right) / 2$ is a closed 1-form on $M$, and $\widehat{\vartheta}$ is its restriction to $\widehat{T X}$. By $(0.1)$,

$$
W=\vartheta \sigma_{\widehat{\vartheta}}^{*} \psi
$$

When $m=\operatorname{dim} X$ is odd, then $W$ is a closed form on $M$. Set $T X^{\perp}=T X /\{\vartheta\}$, and let $e\left(T X^{\perp}\right)$ be the Euler class of $T X^{\perp}$. In this case, by [22, equation (6.20)], the cohomology class $[W]$ of $W$ is given by

$$
[W]=-\frac{1}{2}[\vartheta] \wedge e\left(T X^{\perp}\right),
$$

so that $\int_{X} W$ is the integral over $X$ of a classical cohomology class. When $N$ is arbitrary, there is no clear cohomological interpretation of $W$. 


\subsection{Nondegeneracy, Toeplitz operators, and the spectral gap}

Under our assumptions of nondegeneracy, we show in Theorem 4.4 and in $\S 9.10$ that the lowest eigenvalue of the corresponding Hodge-de Rham Laplacian $D_{p}^{X, 2}$ grows like $p^{2}$. This estimate is obtained by giving a lower bound on the constant term of the Weitzenböck formula for $D_{p}^{X, 2}$, by expressing this constant term as a Toeplitz operator acting on $C^{\infty}\left(N, L^{p}\right)$.

When $G$ is reductive, this is the only use we make of Toeplitz operators. In the general case, which is dealt with in $\S 9$, key results on the algebra of Toeplitz operators play a much more important role. Subtle properties of the Poisson algebra of the fibres $N$, which is a degeneration of the Toeplitz algebra, are used to calculate the $W$-invariant in this more general case. Results by Boutet de Monvel and Guillemin [25] on Toeplitz operators, and further developments by Bordemann, Meinrenken, and Schlichenmaier [23] and Ma and Marinescu $[34,35]$ play an important role in the proofs.

\subsection{Analytic torsion, analytic torsion forms, and $\Gamma$-torsion}

One reason for working with analytic torsion forms instead of the simpler analytic torsion is that the formalism incorporates the variation of the usual analytic torsion. As was shown in [18], the anomaly formulae for the usual Ray-Singer torsion are consequences of basic properties of analytic torsion forms. Also, the analytic torsion forms formalism provides a better control of the local cancellations in the small-time asymptotics of supertraces of heat kernels.

Take the case of one single fibre $X$, and let $\widehat{X}$ denote a $\Gamma$ cover of $X$, where $\Gamma$ is a discrete group. Our nondegeneracy assumption also implies a lower bound for the Hodge Laplacian $D_{p}^{\widehat{X}, 2}$ that grows like $p^{2}$. For $p \in \mathbf{N}$, one can then define the corresponding $\Gamma$ analytic torsion of $\widehat{X}$. Modulo $\mathcal{O}\left(e^{-c p}\right), c>0$, the asymptotics of the $\Gamma$ analytic torsion are shown to be the same as the asymptotics of the analytic torsion itself, in close resemblance with Atiyah's index theorem for coverings [1].

\subsection{Asymptotic torsion and the trace formula}

In $\S 8$, we relate our methods to the Selberg trace formula as used by Müller [43], when $X$ is a compact quotient of a symmetric space. The conditions under which the analytic torsion associated with our flat bundles vanishes, which are stated in Theorem 8.6 and Remark 8.7, are still the ones found by Moscovici and Stanton [40], [11, Chapter 7] for the trivial flat bundle. Also, when $X$ is instead a symmetric space, we use the results of [11] to compute the asymptotics of the semisimple orbital integrals that are associated with the heat operator $\exp \left(-t D_{p}^{X, 2} / 2\right)$. In the case where the flat bundle $F$ is associated with an irreducible representation of $U$ and with the corresponding coadjoint orbit of its highest weight $\lambda$, a condition on $\lambda$ is given in Proposition 8.12 so that it verifies our nondegeneracy condition. Moreover, we compute directly the asymptotics of the orbital integrals as $p \rightarrow+\infty$, and we recover in this special case our asymptotic formula in terms of Berezin integrals. 


\subsection{The main result}

We assume that the nondegeneracy condition is verified. Let $\mathcal{T}_{h}\left(g^{T X}, \nabla^{F_{p}}, g^{F_{p}}\right)$ be the Ray-Singer analytic torsion [44] associated with the de Rham complex $\left(\Omega\left(X, F_{p}\right), d^{X}\right)$. For the precise definition, we refer to (5.49) and (5.50). Set $n=\operatorname{dim} N$. The main result, which is established in Theorems 7.1 and 9.32 , is as follows.

Theorem 0.1. As $p \rightarrow+\infty$,

$$
p^{-n-1} \mathcal{T}_{h}\left(g^{T X}, \nabla^{F_{p}}, g^{F_{p}}\right)=\int_{X} W+\mathcal{O}\left(p^{-1}\right) .
$$

Note that, if $m=\operatorname{dim} X$ is odd, for any $p \in \mathbf{N}, \mathcal{T}_{h}\left(g^{T X}, \nabla^{F_{p}}, g^{F_{p}}\right)$ is a topological invariant, so that all the terms in its asymptotic expansion as $p \rightarrow+\infty$ are topological invariants. Equation (0.4) identifies the first term.

\subsection{The organization of the paper}

This paper is organized as follows. In $\S 1$, we introduce the Berezin integral formalism. Also, given a reductive group $G$, a $G$-bundle $P_{G}$ on $M$, and a compact Kähler manifold $N$, under an assumption of nondegeneracy, we construct natural transgressions of the Euler form of certain vector bundles.

In $\S 2$, given the fibration $\pi: M \rightarrow S$, using the formalism of $\S 1$, we construct the forms $W$ on $M$ and the corresponding invariants $\pi_{*} W$.

In $\S 3$, given a compact Kähler manifold $N$, we express certain operators acting on $H^{(0,0)}(N, L)$ as Toeplitz operators, and we compute the asymptotics of the character for the action of $G$ on $H^{(0,0)}\left(N, L^{p}\right)$ near the identity in $G$.

In $\S 4$, we obtain an asymptotic estimate for the lowest eigenvalue of certain Hodge Laplacians.

In $\S 5$, we summarize the main results of Bismut and Lott [18] on the odd closed superconnection forms on $S$ that are associated with a flat vector bundle on $M$, and the corresponding even analytic torsion forms on $S$.

In $\S 6$, we compute the leading term in the asymptotics as $p \rightarrow+\infty$ of the above odd forms that are associated with $F_{p}$.

In $\S 7$, we obtain our main result, which gives the leading term in the asymptotics as $p \rightarrow+\infty$ of the analytic torsion forms associated with $F_{p}$.

In $\S 8$, we evaluate the asymptotics as $p \rightarrow+\infty$ of certain orbital integrals, and we relate the asymptotics to the more general results that were obtained in $\S \S 6$ and 7 .

Finally, in $\S 9$, we consider the case where $G$ is a general Lie group. We extend the results of $\S \S 1-7$ to this more general case.

In the whole paper, if $E=E_{+} \oplus E_{-}$is a $\mathbf{Z}_{2}$-graded vector space, and $\tau= \pm 1$ defines the $\mathbf{Z}_{2}$-grading, if $A \in \operatorname{End}(E)$, we denote by $\operatorname{Tr}_{\mathrm{s}}[A]$ the supertrace of $A$; i.e.,

$$
\operatorname{Tr}_{\mathrm{s}}[A]=\operatorname{Tr}[\tau A]
$$

The results contained in this paper were announced in [19]. In [19], the normalizations of differential forms by powers of $2 i \pi$ or $2 \pi$ were eliminated for simplicity. 


\section{Multidimensional transgressions of the Euler form}

Let $M$ be a smooth manifold, and let $\left(\widehat{E}, g^{\widehat{E}}, \nabla^{\widehat{E}}\right)$ be a Euclidean vector bundle with connection. Let $G$ be a reductive group with compact maximal subgroup $K$. Let $\mathfrak{g}$ be the Lie algebra of $G$, and let $\mathfrak{g}=\mathfrak{p} \oplus \mathfrak{k}$ be its Cartan decomposition. Let $P_{G}$ be a flat principal $G$-bundle on $M$, let $P_{K}$ be a reduction of $P_{G}$ to a principal $K$-bundle, and let $\mathfrak{g}_{r}=\mathfrak{p}_{r} \oplus \mathfrak{k}_{r}$ be the corresponding bundle of Lie algebras. If $\beta$ is a smooth section of $\widehat{E}^{*} \otimes \mathfrak{p}_{r}$, we construct a family of associated closed forms $a_{t}$ on $M$, which turn out to be a natural extension of forms constructed by Mathai and Quillen [39] when $G=\mathbf{R}$.

This section is organized as follows. In $\S 1.1$, we introduce the reductive group $G$, its compact form $U$, its complexification $G_{\mathbf{C}}$, and the enveloping algebra $U \mathfrak{g}$.

In $\S 1.2$, if $\widehat{E}$ is an Euclidean vector space, we recall elementary results on the Clifford algebras $c(\widehat{E}), \widehat{c}(\widehat{E})$.

In $\S 1.3$, we consider the algebra $U \mathfrak{g} \otimes \widehat{c}(\widehat{E})$.

In $\S 1.4$, if $\mathfrak{u}$ is the Lie algebra of $U$, we consider certain Ad-invariant analytic functions $R(A)$ on $\mathfrak{u}$, and their extension as holomorphic functions on $\mathfrak{g}_{\mathbf{C}}$. In what follows, these functions will be obtained as integrals on a compact complex $U$-manifold $N$ of Duistermaat-Heckman equivariant forms [5, 29, 30].

In $\S 1.5$, we recall simple facts on Berezin integration.

In $\S 1.6$, we introduce the manifold $M$, and the corresponding geometric data, the vector bundle $\widehat{E}$, and the flat principal bundle $P_{G}$.

In $\S 1.7$, over $M$, given a section $\beta$ of $\widehat{E}^{*} \otimes \mathfrak{p}_{r}$, we construct a superconnection $\mathcal{A}_{t}, t \geqslant 0$, with coefficients in $\widehat{c}(\widehat{E}) \otimes U \mathfrak{g}_{r}$. Its principal symbol $\sigma\left(\mathcal{A}_{t}^{2}\right)$ is a section of $\Lambda \cdot\left(\widehat{E}^{*}\right) \otimes S \mathfrak{g}_{r}$.

In $\S 1.8$, we obtain $\sigma\left(\mathcal{A}_{t}^{2}\right)$ from $\mathcal{A}_{t}^{2}$ by a rescaling procedure at the identity along the fibres of $P_{G} \times{ }_{G} G$.

In $\S 1.9$, we establish a Bianchi-like identity for $\sigma\left(\mathcal{A}_{t}^{2}\right)$.

In $\S 1.10$, we define the exponential $\exp \left(-\sigma\left(\mathcal{A}_{t}^{2}\right)\right)$, which should be viewed as a heat operator acting along the fibres of $\mathfrak{g}_{r}$.

In $\S 1.11$, we introduce the Mathai-Quillen forms $\underline{a}_{t}$ on the total space $\widehat{\mathcal{E}}$ of $\widehat{E}$, and the corresponding angle form $-\psi$.

Finally, in $\S 1.12$, we construct the closed forms $a_{t}$ on $M$, and we evaluate these forms in terms of the forms $\underline{a}_{t}$.

\subsection{Reductive groups}

Let $G$ be a connected reductive group, let $\mathfrak{g}$ be its Lie algebra, and let $\Theta$ be the Cartan involution on $G$. Let $K \subset G$ be the maximal compact subgroup of $G$ of the points of $G$ that are fixed by $\Theta$, and let $\mathfrak{k}$ be its Lie algebra. Let $G / K$ be the associated symmetric space. Let

$$
\mathfrak{g}=\mathfrak{p} \oplus \mathfrak{k}
$$

be the Cartan decomposition of $\mathfrak{g}$. Let $B$ be a real-valued nondegenerate bilinear symmetric form on $\mathfrak{g}$ which is invariant under the adjoint action of $G$, and also under $\Theta$. We may and we will assume that \langle\rangle$=-B(\cdot, \Theta \cdot)$ is a $K$-invariant scalar product on $\mathfrak{g}$ that is such that $(1.1)$ is an orthogonal splitting. We denote by || the corresponding norm. 
Let $U$ be the compact form of $G$, and let $\mathfrak{u}=i \mathfrak{p} \oplus \mathfrak{k}$ be its Lie algebra. Let $G_{\mathbf{C}}$ be the complexification of $G$, and let $\mathfrak{g}_{\mathbf{C}}=\mathfrak{g} \otimes_{\mathbf{R}} \mathbf{C}$ be its Lie algebra. Then $G_{\mathbf{C}}$ is also the complexification of $U$, and $\mathfrak{g}_{\mathbf{C}}=\mathfrak{u}_{\mathbf{C}}$. By [32, Proposition 5.6], $G_{\mathbf{C}}$ is still reductive, and $G$ and $U$ are closed subgroups of $G_{\mathbf{C}}$. Also, $U$ is a maximal compact subgroup in $G_{\mathbf{C}}$.

If $E$ is a finite-dimensional complex vector space, and if $\rho: U \rightarrow \operatorname{Aut}(E)$ is a representation of $U$, then $\rho$ extends uniquely to a representation of $G$, and to a holomorphic representation of $G_{\mathbf{C}}$. Also, recall that, by Weyl's unitary trick [32, Proposition 5.7], if $U$ is simply connected, it is equivalent to consider representations of $G$, of $U$ on $E$, or holomorphic representations of $G_{\mathbf{C}}$ on $E$, or representations of $\mathfrak{g}$, of $\mathfrak{u}$, or complex representations of $\mathfrak{g}_{\mathbf{C}}$ on $E$.

Let $U \mathfrak{g}$ denote the enveloping algebras of $\mathfrak{g}$. Then $\mathfrak{g}$ is a Lie subalgebra of $U \mathfrak{g}$. Also, $G$ acts on $U \mathfrak{g}$ by the adjoint representation. Moreover, $U \mathfrak{g}$ can be identified with the algebra of real left-invariant scalar differential operators on $G$. Also, $U \mathfrak{g}$ is equipped with an increasing filtration. Its $\mathrm{Gr}$ is the commutative algebra $S \mathfrak{g}$, the symmetric algebra of $\mathfrak{g}$. Moreover, $U \mathfrak{g}$ and $S \mathfrak{g}$ are canonically isomorphic as $\mathbf{Z}$-graded vector spaces, and this isomorphism induces the identity on $S \mathfrak{g}$. We denote by $\sigma: U \mathfrak{g} \rightarrow S \mathfrak{g}$ the canonical isomorphism, which is also called the symbol map. For instance, if $A, B \in \mathfrak{g}$,

$$
\sigma(A B)=\frac{1}{2}(A B+B A)+\frac{1}{2}[A, B] .
$$

Also, $S \mathfrak{g}$ can be identified with the algebra of real differential operators with constant coefficients on $\mathfrak{g}$.

In what follows, when we want to emphasize that elements of $\mathfrak{g}$ are considered as elements of $U \mathfrak{g}$, and that their products are taken in $U \mathfrak{g}$, they will be underlined. They will not be underlined in the case where just Lie brackets in $\mathfrak{g}$ are considered.

Moreover, $\mathfrak{g}_{\mathbf{C}}$ can be identified with the Lie algebra of holomorphic left-invariant vector fields on $G_{\mathbf{C}}$, and the enveloping algebra $U \mathfrak{g}_{\mathbf{C}}$ with the algebra of left-invariant complex holomorphic differential operators on $G_{\mathbf{C}}$, which acts naturally on holomorphic functions on $G_{\mathbf{C}}$. Similarly, $S_{\mathfrak{g}_{\mathbf{C}}}$ can be identified with the algebra of complex holomorphic differential operators on $\mathfrak{g}_{\mathbf{C}}$ with constant coefficients, which acts naturally on holomorphic functions on $\mathfrak{g}_{\mathbf{C}}$.

Let $E$ be a finite-dimensional complex Hermitian vector space, and let $\rho: U \rightarrow \mathrm{U}(E)$ be a unitary representation. We obtain corresponding representations of $K, G, G_{\mathbf{C}}$. In particular, $\rho$ maps $\mathfrak{k}$ and $\mathfrak{p}$ to skew-adjoint and self-adjoint elements of $\operatorname{End}(E)$.

\subsection{Clifford algebras}

Let $\widehat{E}$ be a real finite-dimensional Euclidean vector space of dimension $m$, and let \langle\rangle be the corresponding scalar product.

Let $c(\widehat{E})$ be the Clifford algebra associated with the bilinear form \langle\rangle . Then $c(\widehat{E})$ is the algebra generated by $1, \widehat{e} \in \widehat{E}$, and the commutation relations

$$
\widehat{e} \widehat{e}^{\prime}+\widehat{e}^{\prime} \widehat{e}=-2\left\langle\widehat{e}, \widehat{e}^{\prime}\right\rangle .
$$

Let $\widehat{c}(\widehat{E})$ be the Clifford algebra associated with the bilinear form -\langle\rangle , i.e., by just changing the sign in the right-hand side of (1.3). If $\widehat{e} \in \widehat{E}$, we denote by $c(\widehat{e}), \widehat{c}(\widehat{e})$ the corresponding elements in $c(\widehat{E}), \widehat{c}(\widehat{E})$. 
The algebras $c(\widehat{E}), \widehat{c}(\widehat{E})$ are $\mathbf{Z}_{2}$-graded. Also, they are equipped with an increasing filtration. The corresponding Gr is just the exterior algebra $\Lambda^{\cdot}(\widehat{E}) \simeq \Lambda^{\cdot}\left(\widehat{E}^{*}\right)$. In what follows, we denote by [] a supercommutator.

If $\widehat{e} \in \widehat{E}$, let $\widehat{e}^{*} \in \widehat{E}^{*}$ correspond to $\widehat{e}$ by the metric. Set

$$
c(\widehat{e})=\widehat{e}^{*} \wedge-i_{\widehat{e}}, \quad \widehat{c}(\widehat{e})=\widehat{e}^{*} \wedge+i_{\widehat{e}} .
$$

If $\widehat{e}, \widehat{f} \in \widehat{E}$, then

$$
[c(\widehat{e}), c(\widehat{f})]=-2\langle\widehat{e}, \widehat{f}\rangle, \quad[\widehat{c}(\widehat{e}), \widehat{c}(\widehat{f})]=2\langle\widehat{e}, \widehat{f}\rangle, \quad[c(\widehat{e}), \widehat{c}(\widehat{f})]=0 .
$$

The maps $\widehat{e} \in \widehat{E} \rightarrow c(\widehat{e}), \widehat{c}(\widehat{e}) \in \operatorname{End}\left(\Lambda^{\cdot}\left(\widehat{E}^{*}\right)\right)$ extend to representations $c: c(\widehat{E}) \rightarrow$ $\operatorname{End}\left(\Lambda^{\cdot}\left(\widehat{E}^{*}\right)\right), \widehat{c}: \widehat{c}(\widehat{E}) \rightarrow \operatorname{End}\left(\Lambda \cdot\left(\widehat{E}^{*}\right)\right)$.

If $H \in \widehat{c}(\widehat{E})$, set

$$
\sigma(H)=\widehat{c}(H) 1 \in \Lambda \cdot\left(\widehat{E}^{*}\right) .
$$

The map $\sigma$ gives a canonical isomorphism of filtered vector spaces $\widehat{c}(\widehat{E}) \simeq \Lambda^{\cdot}\left(\widehat{E}^{*}\right)$ which induces the identity at the level of the Gr. The map $\sigma$ is also called the symbol map.

\subsection{The algebra $\widehat{c}(\widehat{E}) \otimes U \mathfrak{g}$}

The algebra $\widehat{c}(\widehat{E}) \otimes U \mathfrak{g}$ is a $\mathbf{Z}_{2}$-graded algebra, which is equipped with a filtration. Its Gr is the supercommutative algebra $\Lambda^{\cdot}\left(\widehat{E}^{*}\right) \otimes S \mathfrak{g}$. By the above, we get a symbol map $\sigma$ : $\widehat{c}(\widehat{E}) \otimes U \mathfrak{g} \rightarrow \Lambda^{\cdot}\left(\widehat{E}^{*}\right) \otimes S \mathfrak{g}$ which is an identification of filtered $\mathbf{Z}_{2}$-graded vector spaces.

Let $\beta \in \widehat{E}^{*} \otimes \mathfrak{p}$. Then $\beta \in \widehat{E}^{*} \otimes \mathfrak{g}$, and $\beta \in \widehat{E}^{*} \otimes i \mathfrak{u}$. Let $\widehat{e}_{1}, \ldots, \widehat{e}_{m}$ be an orthonormal basis of $\widehat{E}$, and let $\widehat{e}^{1}, \ldots, \widehat{e}^{m}$ be the corresponding dual basis of $\widehat{E}^{*}$. Then

$$
\beta=\sum_{i=1}^{m} \widehat{e}^{i} \beta\left(\widehat{e}_{i}\right) .
$$

Moreover, $\beta^{2} \in \Lambda^{2}\left(\widehat{E}^{*}\right) \otimes \mathfrak{k}$ is given by

$$
\beta^{2}=\frac{1}{2} \widehat{e}^{i} \widehat{e}^{j}\left[\beta\left(\widehat{e}_{i}\right), \beta\left(\widehat{e}_{j}\right)\right]
$$

Let $|\beta|^{2} \in S^{2} \mathfrak{g}$ be given by

$$
|\beta|^{2}=\sum_{i=1}^{m} \beta\left(\widehat{e}_{i}\right)^{2} .
$$

Then $|\beta|^{2} \in S^{2} \mathfrak{g} \cap S^{2} \mathfrak{u}$. Also,

$$
|\beta|^{2}=-|i \beta|^{2} \quad \text { in } S^{2} \mathfrak{g}_{\mathbf{C}} .
$$

Let $\underline{\beta} \in \widehat{E}^{*} \otimes U \mathfrak{g}$ be given by

$$
\underline{\beta}=\sum_{i=1}^{m} \widehat{e}^{i} \underline{\beta}\left(\widehat{e}_{i}\right) .
$$

Then $\underline{\beta}^{2} \in \Lambda^{2}\left(\widehat{E}^{*}\right) \otimes U \mathfrak{g}$ coincides with $\beta^{2}$ in (1.8). Set

$$
|\underline{\beta}|^{2}=\sum_{i=1}^{m} \underline{\beta}\left(\widehat{e}_{i}\right)^{2} .
$$


Then

$$
\sigma\left(|\underline{\beta}|^{2}\right)=|\beta|^{2}
$$

Since $\beta \in \widehat{E}^{*} \otimes i \mathfrak{u},|\underline{\beta}|^{2} \in U \mathfrak{g} \cap U \mathfrak{u}$. More precisely,

$$
|\underline{\beta}|^{2}=-|i \underline{\beta}|^{2} \quad \text { in } U \mathfrak{g}_{\mathbf{C}} .
$$

Let $\widehat{c}(\underline{\beta}) \in \widehat{c}(\widehat{E}) \otimes U \mathfrak{g}$ be given by

$$
\widehat{c}(\underline{\beta})=\sum_{i=1}^{m} \widehat{c}\left(\widehat{e}_{i}\right) \underline{\beta}\left(\widehat{e}_{i}\right) .
$$

Then

$$
\sigma(\widehat{c}(\underline{\beta}))=\beta
$$

\subsection{A complex $G$-manifold and the function $R$}

Recall that $\mathfrak{u}_{\mathbf{C}}=\mathfrak{u} \oplus i \mathfrak{u}$. If $A \in \mathfrak{g}_{\mathbf{C}}=\mathfrak{u}_{\mathbf{C}}$, let $\operatorname{Im} A$ denote the component of $A$ in $i \mathfrak{u}$.

Let $A \in \mathfrak{u} \rightarrow R(A) \in \mathbf{C}$ be a smooth function which is the Fourier transform of a compactly supported finite measure $\Psi$ on $\mathfrak{u}$. Then $R$ extends to a holomorphic function $\mathfrak{g}_{\mathbf{C}} \rightarrow \mathbf{C}$, and moreover there exist $c>0, C>0$ such that, if $A \in \mathfrak{u}_{\mathbf{C}}$,

$$
|R(A)| \leqslant C \exp (c|\operatorname{Im} A|) .
$$

The bounds in (1.17) extend to the derivatives of $R$ of any order. Let $\mathscr{A}$ be the algebra of such functions, and let $\mathscr{A}^{U}$ denote the subalgebra of the $R \in \mathscr{A}$ that are $U$-invariant. Then $\mathscr{A}^{U}$ is also the subalgebra of the $R \in \mathscr{A}$ that are $G_{\mathbf{C}}$-invariant.

Take $\beta \in \widehat{E}^{*} \otimes \mathfrak{p}$ as in $\S 1.3$. Then $-|i \beta|^{2} \in S^{2} \mathfrak{u}$ can be viewed as a nonnegative differential operator on $\mathfrak{u}$ with constant coefficients.

For $t \geqslant 0$, the operator $\exp \left(-t|\beta|^{2}\right)$ acts on $\mathscr{A}$. Let $C^{b}(\mathfrak{u}, \mathbf{C})$ be the vector space of complex-valued continuous functions on $\mathfrak{u}$. By the above, the operator $\exp \left(t|i \beta|^{2}\right)$ also acts on $C^{b}(\mathfrak{u}, \mathbf{C})$. Note that $R(A) \in \mathscr{A} \cap C^{b}(\mathfrak{u}, \mathbf{C})$, and that the actions of $\exp \left(-t|\beta|^{2}\right), \exp \left(t|i \beta|^{2}\right)$ on $R(A)$ coincide.

If $\lambda \in \mathfrak{u}^{*}$, since $\beta \in \widehat{E}^{*} \otimes i \mathfrak{u},\langle\lambda, i \beta\rangle \in \widehat{E}^{*}$. Let $|\langle\lambda, i \beta\rangle|$ denote the norm of $\langle\lambda, i \beta\rangle$ in $\widehat{E}^{*}$. Then $\exp (2 i \pi\langle\lambda, u\rangle) \in \mathscr{A}$, and, moreover,

$$
\exp \left(-t|\beta|^{2}\right) \exp (2 i \pi\langle\lambda, u\rangle)=\exp \left(-4 \pi^{2} t|\langle\lambda, i \beta\rangle|^{2}\right) \exp (2 i \pi\langle\lambda, u\rangle) .
$$

Let $N$ be a compact complex manifold of complex dimension $n$. Let $\mathscr{H}(N)$ be the finite-dimensional Lie algebra of holomorphic vector fields on $N$. Let $\eta$ be a smooth real closed nondegenerate $(1,1)$-form on $N$. We assume that the group $U$ acts holomorphically on $N$ and preserves the form $\eta$. If $A \in \mathfrak{u}$, let $A^{N} \in \mathscr{H}(N)$ be the corresponding holomorphic vector field on $N$, so that $A \in \mathfrak{u} \rightarrow-A^{N} \in \mathscr{H}(N)$ is a morphism of Lie algebras. Also, we assume there is a moment map $\mu: N \rightarrow \mathfrak{u}^{*}$ attached to this action such that, if $u \in U$, then

$$
\mu(u x)={ }^{t} \operatorname{Ad}^{-1}(u) \mu(x),
$$

and, moreover, if $A \in \mathfrak{u}$,

$$
d\langle\mu, A\rangle-i_{A^{N}} \eta=0
$$


By (1.19), (1.20), we deduce that, if $A, B \in \mathfrak{u}$,

$$
\langle\mu,[A, B]\rangle=\eta\left(A^{N}, B^{N}\right) .
$$

Equation (1.20) is equivalent to

$$
\bar{\partial}\langle\mu, A\rangle-i_{A^{N(1,0)}} \eta=0 .
$$

Classically, the action of $U$ extends to a holomorphic action of $G_{\mathbf{C}}$ on $N$. If $A \in \mathfrak{g}_{\mathbf{C}}$, the corresponding vector field $A^{N} \in \mathscr{H}(N)$ is still characterized by (1.22).

If $A \in \mathfrak{u}$, set

$$
R(A)=\int_{N} \exp (2 i \pi\langle\mu, A\rangle+\eta) .
$$

By (1.23), we find that $R \in \mathscr{A}^{U}$. Also, if $A \in \mathfrak{u}, R(A)$ can be computed using the localization formulae of $[5,29,30]$. By (1.18), (1.23), we obtain

$$
\exp \left(-t|\beta|^{2}\right) R(A)=\int_{N} \exp \left(-4 \pi^{2} t|\langle\mu, i \beta\rangle|^{2}+2 i \pi\langle\mu, A\rangle+\eta\right) .
$$

Definition 1.1. Let $\sigma_{\beta}$ be the smooth section of $\widehat{E}^{*}$ on $N$,

$$
\sigma_{\beta}=2 \pi\langle\mu, i \beta\rangle
$$

Then $\beta$ is said to be nondegenerate if $\sigma_{\beta}$ does not vanish on $N$. Equivalently, there exists $a>0$ such that

$$
\left|\sigma_{\beta}\right|^{2} \geqslant a
$$

By (1.24)-(1.26), if $\beta$ is nondegenerate, there exist $c>0, C>0$ such that, if $A \in \mathfrak{u}_{\mathbf{C}}$,

$$
\left|\exp \left(-t|\beta|^{2}\right) R(A)\right| \leqslant C \exp (-t a+c|\operatorname{Im} A|) .
$$

\subsection{The Berezin integral}

Let $V$ be a real finite-dimensional vector space of dimension $m^{\prime}$.

Let $\widehat{e}_{1}, \ldots, \widehat{e}_{m}$ be an orthonormal basis of $\widehat{E}$, and let $\widehat{e}^{1}, \ldots, \widehat{e}^{m}$ be the corresponding dual basis of $\widehat{E}^{*}$. Suppose that $\widehat{E}$ is oriented and that $\widehat{e}_{1}, \ldots, \widehat{e}_{m}$ is an oriented basis of $\widehat{E}$. Let $\int^{\widehat{B}}$ be the linear map from $\Lambda^{\cdot}\left(V^{*}\right) \widehat{\otimes} \Lambda^{\cdot}\left(\widehat{E}^{*}\right)$ into $\Lambda^{\cdot}\left(V^{*}\right)$ such that, if $\alpha \in$ $\Lambda^{\cdot}\left(V^{*}\right), \alpha^{\prime} \in \Lambda\left(\widehat{E}^{*}\right)$

$$
\begin{aligned}
& \int_{\widehat{B}}^{\widehat{B}} \alpha \alpha^{\prime}=0 \quad \text { if } \operatorname{deg} \alpha^{\prime}<m, \\
& \int^{\widehat{B}} \alpha \widehat{\boldsymbol{e}}^{1} \wedge \cdots \wedge \widehat{e}^{m}=\frac{(-1)^{m(m+1) / 2}}{\pi^{m / 2}} \alpha .
\end{aligned}
$$

More generally, let $o(\widehat{E})$ be the orientation line of $\widehat{E}$. Then $\int^{\widehat{B}}$ defines a linear map from $\Lambda^{\cdot}\left(V^{*}\right) \widehat{\otimes} \Lambda^{\cdot}\left(\widehat{E}^{*}\right)$ into $\Lambda^{\cdot}\left(V^{*}\right) \widehat{\otimes} o(\widehat{E})$, which is called a Berezin integral.

Let $A$ be an antisymmetric endomorphism of $\widehat{E}$. We identify $A$ with $\omega_{A} \in \Lambda^{2}\left(\widehat{E}^{*}\right)$ that is given by

$$
\omega_{A}=\frac{1}{2} \sum_{1 \leqslant i, j \leqslant m}\left\langle\widehat{e}_{i}, A \widehat{e}_{j}\right\rangle \widehat{e}^{i} \wedge \widehat{e}^{j}
$$


By definition, the Pfaffian Pf $\left[\frac{A}{2 \pi}\right]$ of $\frac{A}{2 \pi}$ is given by

$$
\int^{\widehat{B}} \exp \left(-\omega_{A} / 2\right)=\operatorname{Pf}\left[\frac{A}{2 \pi}\right] .
$$

Then $\operatorname{Pf}\left[\frac{A}{2 \pi}\right]$ vanishes if $m$ is odd. Moreover,

$$
\operatorname{det}\left[\frac{A}{2 \pi}\right]=\operatorname{Pf}\left[\frac{A}{2 \pi}\right]^{2} .
$$

\subsection{The manifold $M$}

Let $M$ be a smooth manifold. Let $p: P_{G} \rightarrow M$ be a principal $G$-bundle. From the embedding $G \rightarrow G_{\mathbf{C}}$, we get a corresponding principal $G_{\mathbf{C}}$-bundle $p: P_{G_{\mathbf{C}}} \rightarrow M$.

Since $G / K$ is contractible, there are smooth sections of the fibre bundle $P_{G} \times{ }_{G} G / K$. Let $\mathcal{S}$ be the set of these smooth sections. Then $\mathcal{S}$ is an infinite-dimensional connected Fréchet manifold.

Let $h \in \mathcal{S}$. To $h$ corresponds a reduction of the principal $G$-bundle $p: P_{G} \rightarrow M$ to a principal $K$-bundle $p: P_{K} \rightarrow M$. From the embedding $K \rightarrow U$, we obtain the associated principal $U$-bundle $P_{U}$. More generally, on $M \times \mathcal{S}$, the $G$-bundle $P_{G}$ can be reduced to a $K$-bundle $P_{K}$.

Since $G$ acts on the right on $P_{G}, U \mathfrak{g}$ maps into differential operators acting on the right along the fibre of $P_{G}$.

From the action of $G$ on itself by conjugation, we get the bundle of Lie groups

$$
G_{r}=P_{G} \times_{G} G .
$$

The corresponding bundle of Lie algebras $\mathfrak{g}_{r}$ is given by

$$
\mathfrak{g}_{r}=P_{G} \times_{G} \mathfrak{g} .
$$

Moreover, $B$ induces a corresponding bilinear form on $\mathfrak{g}_{r}$. Also, $G_{r}$ acts on the left on $P_{G}$ by an action which commutes with the right action of $G$ on $P_{G}$. The bundle of enveloping algebras

$$
U \mathfrak{g}_{r}=P_{G} \times_{G} U \mathfrak{g}
$$

can be identified with the algebra of right-invariant differential operators along the fibres of $G_{r}$. Equivalently, it can be identified with the algebra of differential operators along the fibres of $P_{G}$ which are invariant under the right action of $G$. For the identification to preserve the Lie bracket, $A \in \mathfrak{g}_{r}$ maps into the vector field associated with $-A$ along the fibre of $P_{G}$.

Since

$$
\mathfrak{g}_{r}=P_{K} \times_{K} \mathfrak{g},
$$

the scalar product of $\mathfrak{g}$ induces a scalar product on $\mathfrak{g}_{r}$, and the splitting (1.1) of $\mathfrak{g}$ induces the corresponding splitting of $\mathfrak{g}_{r}$,

$$
\mathfrak{g}_{r}=\mathfrak{p}_{r} \oplus \mathfrak{k}_{r}
$$


In what follows, we assume that $P_{G}$ is equipped with a flat connection. Let $\theta^{\mathfrak{g}}$ denote the $\mathfrak{g}$-valued connection form on $P_{G}$. By projection of $\theta^{\mathfrak{g}}$ on $\mathfrak{k}$ with respect to the Cartan decomposition (1.1), we get a connection form $\theta^{\mathfrak{k}}$ on $P_{K}$. If $\theta^{\mathfrak{p}}$ is the projection of $\theta^{\mathfrak{g}}$ on $\mathfrak{p}$, we have the identity

$$
\theta^{\mathfrak{g}}=\theta^{\mathfrak{p}}+\theta^{\mathfrak{k}}
$$

Similarly, from $\theta^{\mathfrak{g}}$, we get connection forms $\theta^{\mathfrak{u}}, \theta^{\mathfrak{g} \mathbf{C}}$ on $P_{U}, P_{G_{\mathbf{C}}}$. In what follows, $\theta^{\mathfrak{p}}$ will be considered as a section of $T^{*} M \otimes \mathfrak{p}_{r}$.

Let $\Theta^{\mathfrak{k}}$ be the curvature of the connection $\theta^{\mathfrak{k}}$. Since $\theta^{\mathfrak{g}}$ is flat, from (1.37), we get

$$
\Theta^{\mathfrak{k}}=-\frac{1}{2}\left[\theta^{\mathfrak{p}}, \theta^{\mathfrak{p}}\right], \quad\left[d+\theta^{\mathfrak{k}}, \theta^{\mathfrak{p}}\right]=0 .
$$

In what follows, we will write the first equation in (1.38) in the form

$$
\Theta^{\mathfrak{k}}=-\theta^{\mathfrak{p}, 2} \text {. }
$$

Moreover,

$$
\left[\theta^{\mathfrak{p}}, \theta^{\mathfrak{p}, 2}\right]=0 \text {. }
$$

Let $\nabla^{\mathfrak{g}_{r}, u}$ be the connection on $\mathfrak{g}_{r}$ which is induced by the connection $\theta^{\mathfrak{k}}$. This connection preserves the splitting (1.36) of $\mathfrak{g}_{r}$. Moreover, the second equation in (1.38) can be written in the form

$$
\nabla^{\mathfrak{g}_{r}, u} \theta^{\mathfrak{p}}=0 .
$$

The curvature $\Theta^{\mathfrak{u}}$ of the connection $\theta^{\mathfrak{u}}$ is given by

$$
\Theta^{\mathfrak{u}}=-2 \theta^{\mathfrak{p}, 2} .
$$

\subsection{A superconnection over $M$}

Let $\left(\widehat{E}, g^{\widehat{E}}\right)$ be a real Euclidean vector bundle of dimension $m$ on $M$, which is equipped with an Euclidean connection $\nabla^{\widehat{E}}$, whose curvature is denoted $R^{\widehat{E}}$.

Let $e\left(\widehat{E}, \nabla^{\widehat{E}}\right)$ be the Euler form of $\left(\widehat{E}, \nabla^{\widehat{E}}\right)$, and let $e(\widehat{E})$ be the corresponding Euler class. Then

$$
e\left(\widehat{E}, \nabla^{\widehat{E}}\right)=\operatorname{Pf}\left[\frac{R^{\widehat{E}}}{2 \pi}\right] .
$$

By (1.30), we can rewrite (1.43) in the form

$$
e\left(\widehat{E}, \nabla^{\widehat{E}}\right)=\int^{\widehat{B}} \exp \left(-\frac{1}{4}\left\langle\widehat{e}_{i}, R^{\widehat{E}} \widehat{e}_{j}\right) \widehat{e}^{i} \widehat{e}^{j}\right) .
$$

We define the bundle of Clifford algebras $\widehat{c}(\widehat{E})$ as in $\S 1.2$, and we use the corresponding notation.

Let $Q$ be the $\mathrm{O}(m)$-bundle of orthogonal frames in $\widehat{E}$. Then $Q$ is equipped with an so $(m)$-valued connection form $\theta^{\text {so }(m)}$. Since so $(m)$ embeds as a Lie subalgebra of $\widehat{c}^{\text {even }}\left(\mathbf{R}^{m}\right)$, it will be convenient to view $\theta^{\operatorname{so}(m)}$ as taking values in $\widehat{c}^{\text {even }}\left(\mathbf{R}^{m}\right)$.

Set

$$
R_{G}=P_{G} \times{ }_{M} Q
$$


Then $R_{G}$ is a $G \times \mathrm{O}(m)$-bundle, and $\theta^{\mathfrak{k}}+\theta^{\operatorname{so}(m)}$ is a connection form on $R_{G}$. Of course, $G$ can be replaced by any of the groups already considered in $\S 1.1$. Let $\nabla \widehat{E} \otimes \mathfrak{g}_{r}, u$ be the connection on $\widehat{E} \otimes \mathfrak{g}_{r}$ that is induced by $\nabla^{\widehat{E}}, \nabla^{\mathfrak{g}_{r}, u}$. We still denote by $\nabla^{\widehat{E} \otimes \mathfrak{g}_{r}, u}$ the corresponding connection on $\widehat{c}(\widehat{E}) \otimes U \mathfrak{g}_{r}$.

Let $\beta$ be a smooth section of $\widehat{E}^{*} \otimes \mathfrak{p}_{r}$. We will use the notation of $\S 1.3$. In particular, $\widehat{e_{1}}, \ldots, \widehat{e}_{m}$ is an orthonormal basis of $\widehat{E}$, and $\widehat{e}^{1}, \ldots, \widehat{e}^{m}$ is the dual basis. Then $\beta^{2}$ in (1.8) is now a section of $\Lambda^{2}\left(\widehat{E}^{*}\right) \otimes \mathfrak{k}_{r}$, and $|\beta|^{2}$ in (1.9) is a section of $S^{2} \mathfrak{g}_{r} \cap S^{2} \mathfrak{u}_{r}$. Moreover, $\widehat{c}(\underline{\beta})$ is a section of $\widehat{c}(\widehat{E}) \otimes U \mathfrak{g}_{r}$.

Definition 1.2. For $t \geqslant 0$, let $\mathcal{A}_{t}$ be the superconnection

$$
\mathcal{A}_{t}=\nabla^{\widehat{E} \otimes \mathfrak{g}_{r}, u}+\sqrt{t} \widehat{c}(\underline{\beta}) .
$$

Then $\mathcal{A}_{t}$ is just the superconnection $\mathcal{A}_{1}$ which is associated with the metric $g^{\widehat{E}} / t$. The curvature $\mathcal{A}_{t}^{2}$ is a smooth section of $\left[\Lambda^{*}\left(T^{*} M\right) \widehat{\otimes} \widehat{c}(\widehat{E})\right]^{\text {even }} \otimes U \mathfrak{g}_{r}$, and $\sigma\left(\mathcal{A}_{t}^{2}\right)$ is a section of $\left[\Lambda^{\cdot}\left(T^{*} M\right) \widehat{\otimes} \Lambda^{\cdot}\left(\widehat{E}^{*}\right)\right]^{\text {even }} \otimes S \mathfrak{g}_{r}$.

Theorem 1.3. The following identities hold:

$$
\begin{aligned}
& \mathcal{A}_{t}^{2}=\frac{1}{4}\left\langle\widehat{e}_{i}, R^{\widehat{E}} \widehat{e}_{j}\right\rangle \widehat{c}\left(\widehat{e}_{i}\right) \widehat{c}\left(\widehat{e}_{j}\right)-\theta^{\mathfrak{p}, 2}-\sqrt{t} \widehat{c}\left(\widehat{e}_{i}\right) \nabla_{.}^{\widehat{E} \otimes \mathfrak{g}_{r}, u} \beta\left(\widehat{e}_{i}\right) \\
& +t|\underline{\beta}|^{2}+\frac{t}{2} \widehat{c}\left(\widehat{e}_{i}\right) \widehat{c}\left(\widehat{e}_{j}\right) \beta^{2}\left(\widehat{e}_{i}, \widehat{e}_{j}\right), \\
& \sigma\left(\mathcal{A}_{t}^{2}\right)=\frac{1}{4}\left\langle\widehat{e}_{i}, R^{\widehat{E}} \widehat{e}_{j}\right\rangle \widehat{e}^{i} \widehat{e}^{j}-\theta^{\mathfrak{p}, 2}+\sqrt{t} \nabla_{.}^{\widehat{E} \otimes \mathfrak{g}_{r}, u} \beta+t|\beta|^{2}+t \beta^{2} .
\end{aligned}
$$

Proof. By (1.39), we get

$$
\nabla^{\widehat{E} \otimes \mathfrak{g}_{r}, u, 2}=\frac{1}{4}\left\langle\widehat{e}_{i}, R^{\widehat{E}} \widehat{e}_{j}\right\rangle \widehat{c}\left(\widehat{e}_{i}\right) \widehat{c}\left(\widehat{e}_{j}\right)-\theta^{\mathfrak{p}, 2} .
$$

The first identity in (1.47) follows from (1.46), (1.48). By applying the symbol map to this identity, and using (1.13), we get the second identity.

We will write $\sigma\left(\mathcal{A}_{t}^{2}\right)$ as a sum of components of degree 0 to 2 in $S \mathfrak{g}_{r}$; i.e.,

$$
\sigma\left(\mathcal{A}_{t}^{2}\right)=\sum_{i=0}^{2} \sigma\left(\mathcal{A}_{t}^{2}\right)^{(i)} .
$$

By (1.47) and (1.49), we get

$$
\begin{aligned}
& \sigma\left(\mathcal{A}_{t}^{2}\right)^{(0)}=\frac{1}{4}\left\langle\widehat{e}_{i}, R^{\widehat{E}} \widehat{e}_{j}\right\rangle \widehat{e}^{i} \widehat{e}^{j}, \\
& \sigma\left(\mathcal{A}_{t}^{2}\right)^{(1)}=-\theta^{\mathfrak{p}, 2}+\sqrt{t} \nabla^{\widehat{E} \otimes \mathfrak{g}_{r}, u} \beta+t \beta^{2}, \\
& \sigma\left(\mathcal{A}_{t}^{2}\right)^{(2)}=t|\beta|^{2} .
\end{aligned}
$$




\subsection{The principal symbol as a rescaled limit}

If $\widehat{e} \in \widehat{E}$, the action of $\widehat{c}(\widehat{e})$ on $\Lambda^{*}\left(\widehat{E}^{*}\right)$ was defined in (1.4). We will not distinguish $\widehat{c}(\widehat{e})$ from its action on $\Lambda \cdot\left(\widehat{E}^{*}\right)$.

For $a>0$, let $\psi_{a}$ be the automorphism of $\Lambda^{\cdot}\left(T^{*} M\right)$ that is such that, if $\alpha \in \Lambda^{q}\left(T^{*} M\right)$, then

$$
\psi_{a} \alpha=a^{q} \alpha
$$

For $s>0, t>0$, set

$$
\mathcal{A}_{s, t}=\psi_{\sqrt{s}} \mathcal{A}_{s^{2} t} \psi_{1 / \sqrt{s}}
$$

Let $N^{\Lambda^{\cdot}\left(\widehat{E}^{*}\right)}$ be the number operator of $\Lambda^{\cdot}\left(\widehat{E}^{*}\right)$. For $s>0, \widehat{e} \in \widehat{E}$, set

$$
\widehat{c_{S}}(\widehat{e})=s^{-N^{\Lambda^{*}\left(\widehat{E}^{*}\right)} / 2} \widehat{c}(\widehat{e}) s^{N^{\Lambda^{\cdot}\left(\widehat{E}^{*}\right)} / 2} \text {. }
$$

Then

$$
\widehat{c}_{s}(\widehat{e})=\frac{1}{\sqrt{s}} \widehat{e}^{*} \wedge+\sqrt{s} i_{\widehat{e}} .
$$

Set

$$
\mathcal{B}_{s, t}=s^{-N^{\Lambda^{\cdot}\left(\widehat{E}^{*}\right)} / 2} \mathcal{A}_{s, t} s^{N^{\Lambda^{\cdot}\left(\widehat{E}^{*}\right)} / 2} .
$$

By (1.47), (1.52), and (1.53), we get

$$
\begin{aligned}
\mathcal{B}_{s, t}^{2}= & \frac{s}{4}\left\langle\widehat{e}_{i}, R^{\widehat{E} \widehat{e}_{j}}\right\rangle \widehat{c}_{s}\left(\widehat{e}_{i}\right) \widehat{c}_{s}\left(\widehat{e}_{j}\right)-s \theta^{\mathfrak{p}, 2}-\sqrt{s t} \widehat{c}_{s}\left(\widehat{e}_{i}\right) \nabla^{\widehat{E} \otimes \mathfrak{g}_{r}, u} s \beta\left(\widehat{e}_{i}\right) \\
& +t s^{2}|\underline{\beta}|^{2}+\frac{t s}{2} \widehat{c}_{s}\left(\widehat{e}_{i}\right) \widehat{c}_{s}\left(\widehat{e}_{j}\right) s \beta^{2}\left(\widehat{e}_{i}, \widehat{e}_{j}\right) .
\end{aligned}
$$

By identifying $U \mathfrak{g}_{r}$ to the algebra of right-invariant differential operators on $G_{r}, \mathcal{B}_{s, t}^{2}$ can be viewed as a differential operator along the fibres of $G_{r}$. Similarly, by identifying $S \mathfrak{g}_{r}$ to the algebra of differential operators with constant coefficients on $\mathfrak{g}_{r}, \sigma\left(\mathcal{A}_{t}^{2}\right)$ can be viewed as a differential operator along the fibres of $\mathfrak{g}_{r}$.

We use the exponential map to identify a neighbourhood of 0 in $\mathfrak{g}_{r}$ with a neighbourhood of $1 \in G_{r}$. For $a \in \mathbf{R}$, if $h \in C^{\infty}(\mathfrak{g}, \mathbf{C})$, put

$$
\delta_{a} h(f)=h(a f) .
$$

Of course $\delta_{a}$ also acts fibrewise on $C^{\infty}\left(\mathfrak{g}_{r}, \mathbf{C}\right)$.

For $s>0, t>0$, set

$$
\mathcal{C}_{s, t}=\delta_{s} \mathcal{B}_{s, t} \delta_{1 / s}
$$

Then $\mathcal{C}_{s, t}$ can be viewed as a differential operator defined on a neighbourhood of $0 \in \mathfrak{g}$. The same is true for $\mathcal{C}_{s, t}^{2}$.

We will say that a sequence of differential operators with smooth coefficients on $\mathfrak{g}_{r}$ converges if the corresponding coefficients converge uniformly on compact sets of the total space of $\mathfrak{g}_{r}$ together with their derivatives of any order.

Proposition 1.4. As $s \rightarrow 0$,

$$
\mathcal{C}_{s, t}^{2} \rightarrow \sigma\left(\mathcal{A}_{t}^{2}\right)
$$

Proof. We use equations (1.47), (1.54), and (1.56), and we get (1.59). 


\subsection{The Bianchi identity}

If $A \in \mathfrak{g}_{r}, A$ and $\operatorname{ad}(A)$ act on $S \mathfrak{g}_{r}$, respectively increasing the degree by 1 and preserving the degree. We use the notation

$$
\operatorname{ad}(\beta)=\sum_{i=1}^{m} \widehat{e}^{i} \operatorname{ad}\left(\beta\left(\widehat{e}_{i}\right)\right), \quad i_{\beta}=\sum_{i=1}^{m} \beta\left(\widehat{e}_{i}\right) i_{\widehat{e}_{i}} .
$$

These operators act as odd derivations of the algebra $\Lambda^{\cdot}\left(T^{*} M\right) \widehat{\otimes} \Lambda^{\cdot}\left(\widehat{E}^{*}\right) \otimes S \mathfrak{g}_{r}$. We still denote by $\nabla^{\widehat{E} \otimes \mathfrak{g}_{r}, u}$ the obvious connection on $\Lambda^{\cdot}\left(\widehat{E}^{*}\right) \otimes S \mathfrak{g}_{r}$.

Definition 1.5. Let $\mathcal{L}_{t}$ be the differential operator

$$
\mathcal{L}_{t}=\nabla^{\widehat{E} \otimes \mathfrak{g}_{r}, u}+2 i_{\sqrt{t} \beta}+\operatorname{ad}(\sqrt{t} \beta)
$$

Theorem 1.6. The following identities hold:

$$
\mathcal{L}_{t} \sigma\left(\mathcal{A}_{t}^{2}\right)=0, \quad \frac{\partial}{\partial t} \sigma\left(\mathcal{A}_{t}^{2}\right)=\mathcal{L}_{t} \frac{\beta}{2 \sqrt{t}} .
$$

Proof. We use equation (1.47) for $\sigma\left(\mathcal{A}_{t}^{2}\right)$. We grade the left-hand side of (1.62) by its degree in $\sqrt{t}$. In degree 0 , the first identity in (1.62) is trivial. The coefficient of $\sqrt{t}$ is given by

$$
-R^{\widehat{E}} \beta+R^{\widehat{E}} \beta-\left[\beta, \theta^{\mathfrak{p}, 2}\right]-\left[\theta^{\mathfrak{p}, 2}, \beta\right]=0 .
$$

The coefficient of $t$ is given by

$$
2 \beta\left(\widehat{e}_{i}\right) \nabla^{\widehat{E} \otimes \mathfrak{g}_{r}, u} \beta\left(\widehat{e}_{i}\right)-\left[\beta, \nabla^{\widehat{E} \otimes \mathfrak{g}_{r}, u} \beta\right]-2 \beta\left(\widehat{e}_{i}\right) \nabla^{\widehat{E} \otimes \mathfrak{g}_{r}, u} \beta\left(\widehat{e}_{i}\right)+\left[\beta, \nabla^{\widehat{E} \otimes \mathfrak{g}_{r}, u} \beta\right]=0 .
$$

Clearly,

$$
\operatorname{ad}(\beta) \beta^{2}=0
$$

By (1.65), the coefficient of $t^{3 / 2}$ is given by

$$
\operatorname{ad}(\beta)|\beta|^{2}+2 i_{\beta} \beta^{2}=0 .
$$

This completes the proof of the first identity in (1.62).

By (1.47), we get

$$
\frac{\partial}{\partial t} \sigma\left(\mathcal{A}_{t}^{2}\right)=\frac{1}{2 \sqrt{t}} \nabla^{\widehat{E} \otimes \mathfrak{g}_{r}, u} \beta+|\beta|^{2}+\beta^{2},
$$

which is equivalent to the second identity in (1.62). The proof of our theorem is completed.

Remark 1.7. When $G=\mathbf{R},(1.62)$ is an identity of Mathai and Quillen [39, §6]. The second identity in (1.62) is a consequence of the first one. Indeed, replace $M$ by $M \times \mathbf{R}_{+}^{*}$. Over $M \times\{t\}$, we replace $\beta$ by $\sqrt{t} \beta$. Let $\underline{\mathcal{A}}_{1}$ be the analogue of $\mathcal{A}_{1}$ over the enlarged space. Then one has the easy identity,

$$
\sigma\left(\underline{\mathcal{A}}_{1}^{2}\right)=\sigma\left(\mathcal{A}_{t}^{2}\right)+\frac{d t}{2 \sqrt{t}} \beta .
$$


By the first identity in (1.62), we get

$$
\left(\nabla^{\widehat{E} \otimes \mathfrak{g}_{r}, u}+d t \frac{\partial}{\partial t}+2 i_{\sqrt{t} \beta}+\operatorname{ad}(\sqrt{t} \beta)\right) \sigma\left(\underline{\mathcal{A}}_{1}^{2}\right)=0,
$$

which gives both identities in (1.62).

Another more geometric approach which we will use later is to keep $\beta$ fixed, and, over the fibre $M \times\{t\}$, to equip $\widehat{E}$ with the metric $g \widehat{E} / t$. We obtain in this way a new metric on $\widehat{E}$ over $M \times \mathbf{R}_{+}^{*}$. Then $\nabla^{\widehat{E}}+d t\left(\frac{\partial}{\partial t}-\frac{1}{2 t}\right)$ is a Euclidean connection on $\widehat{E}$. We trivialize $\widehat{E}$ over $\mathbf{R}_{+}^{*}$ by parallel transport with respect to this Euclidean connection. The ultimate effect is that, once this trivialization is done, $\beta$ is replaced by $\sqrt{t} \beta$. We can now proceed as before.

\subsection{The exponential of $\sigma\left(\mathcal{A}_{t}^{2}\right)$}

Let $\bar{S} \mathfrak{g}_{r}$ be the formal completion of $S \mathfrak{g}_{r}$. The exponential $\exp \left(-\sigma\left(\mathcal{A}_{t}^{2}\right)\right)$ lies naturally in $\left(\Lambda^{\cdot}\left(T^{*} M\right) \widehat{\otimes} \Lambda^{\cdot}\left(\widehat{E}^{*}\right)\right)^{\text {even }} \otimes \bar{S} \mathfrak{g}_{r}$. By $(1.49)$, we get

$$
\exp \left(-\sigma\left(\mathcal{A}_{t}^{2}\right)\right)=\prod_{i=0}^{2} \exp \left(-\sigma\left(\mathcal{A}_{t}^{2}\right)^{(i)}\right) \text {. }
$$

Because, for $0 \leqslant i \leqslant 1, \sigma\left(\mathcal{A}_{t}^{2}\right)^{(i)}$ is of positive degree in $\Lambda^{*}\left(T^{*} M\right) \widehat{\otimes} \Lambda^{\cdot}\left(\widehat{E}^{*}\right)$, the corresponding exponential just contains a finite number of terms, and they lie in $\Lambda^{\cdot}\left(T^{*} M\right) \widehat{\otimes} \Lambda^{\cdot}\left(\widehat{E}^{*}\right) \otimes S \mathfrak{g}_{r}$. By $(1.50)$,

$$
\exp \left(-\sigma\left(\mathcal{A}_{t}^{2}\right)^{(2)}\right)=\exp \left(-t|\beta|^{2}\right) \text {. }
$$

The proper interpretation of $\exp \left(-t|\beta|^{2}\right)$ as an operator has been given in $\S 1.4$. In particular, $\exp \left(-\sigma\left(\mathcal{A}_{t}^{2}\right)\right)$ acts naturally on the algebra $\mathscr{A}^{U}$. More precisely, if $R \in \mathscr{A}^{U}$, then $\exp \left(-\sigma\left(\mathcal{A}_{t}^{2}\right)\right) R(0) \in \Lambda^{\cdot}\left(T^{*} M\right) \widehat{\otimes} \Lambda^{\cdot}\left(\widehat{E}^{*}\right)$.

\subsection{The Mathai-Quillen forms $\underline{a}_{t}, \underline{b}_{t}$}

In what follows, we identify $\widehat{E}$ and $\widehat{E}^{*}$ by the metric $g \widehat{E}$.

We follow Mathai and Quillen [39]. Let $\tau: \widehat{\mathcal{E}} \rightarrow M$ be the total space of the vector bundle $\widehat{E}$ on $M$. Let $\widehat{Y}$ denote the tautological section of $\tau^{*} \widehat{E}$ on $\widehat{\mathcal{E}}$. Then $\tau^{*} \nabla^{\widehat{E}} \widehat{Y}$ is a section of $\Lambda^{\cdot}\left(T^{*} \widehat{\mathcal{E}}\right) \otimes \tau^{*} \widehat{E}$. Let $o(\widehat{E})$ be the orientation bundle of $\widehat{E}$.

For $t \geqslant 0$, set

$$
A_{t}=\frac{1}{4}\left\langle\widehat{e}_{i}, R^{\widehat{E}} \widehat{e}_{j}\right) \widehat{e}^{i} \widehat{e}^{j}+\tau^{*} \nabla^{\widehat{E}} \sqrt{t} \widehat{Y}+t|\widehat{Y}|^{2} .
$$

By $[39, \S 6]$, we get

$$
\left(\tau^{*} \nabla^{\widehat{E}}+2 i_{\sqrt{t} \widehat{Y}}\right) A_{t}=0, \quad \frac{\partial}{\partial t} A_{t}=\left(\tau^{*} \nabla^{\widehat{E}}+2 i_{\sqrt{t} \widehat{Y}}\right) \frac{\widehat{Y}}{2 \sqrt{t}} .
$$

The identities in (1.73) are special cases of the ones in Theorem 1.6.

Definition 1.8. Let $\underline{a}_{t}, \underline{b}_{t}$ be the forms on $\widehat{\mathcal{E}}$ with values in $o(\widehat{E})$,

$$
\underline{a}_{t}=\int^{\widehat{B}} \exp \left(-A_{t}\right), \quad \underline{b}_{t}=\int^{\widehat{B}} \frac{\widehat{Y}}{2 \sqrt{t}} \exp \left(-A_{t}\right) .
$$


By Mathai and Quillen $[39, \S 6]$, the form $\underline{a}_{t}$ is of degree $m$, it is closed, and its cohomology class does not depend on $t$, and is equal to $\tau^{*} e(\widehat{E})$. Also, the form $\underline{b}_{t}$ is of degree $m-1$. Finally, by [39, §7], [22, Theorem 3.4], for $t>0$, we have the identity

$$
\frac{\partial}{\partial t} \underline{a}_{t}=-d \underline{b}_{t}
$$

The above properties of $\underline{a}_{t}, \underline{b}_{t}$ are easy consequences of (1.73).

We embed $M$ into $\widehat{\mathcal{E}}$ as the zero set of $\widehat{Y}$. Let $\delta_{M}$ be the current of integration on $M$. If $K$ is a compact subset of $\widehat{\mathcal{E}}$, let \|\|$_{C_{K}^{1}(\widehat{\mathcal{E}})}$ be a natural norm on the Banach space of $C^{1}$ forms on $\widehat{\mathcal{E}}$ with coefficients in the orientation bundle $o(T M)$ of $T M$ with support included in $K$. By [22, Theorem 3.5], if $K$ is a compact subset of $\widehat{\mathcal{E}}$, there exists $C>0$ such that, if $v$ is a smooth form on $\widehat{\mathcal{E}}$ with values in $o(T M)$, for $t \geqslant 1$,

$$
\left|\int_{\widehat{\mathcal{E}}} v\left(\underline{a}_{t}-\delta_{M}\right)\right| \leqslant \frac{C}{\sqrt{t}}\|v\|_{C_{K}^{1}(\widehat{\mathcal{E}})}, \quad\left|\int_{\widehat{\mathcal{E}}} \nu \underline{b}_{t}\right| \leqslant \frac{C}{t^{3 / 2}}\|v\|_{C_{K}^{1}(\widehat{\mathcal{E}})} .
$$

Now, we follow [22, Definition 3.6].

Definition 1.9. Let $\psi$ be the current on $\widehat{\mathcal{E}}$ with values in $o(\widehat{E})$,

$$
\psi=\int_{0}^{+\infty} \underline{b}_{t} d t
$$

The restriction of the current $\psi$ to the sphere bundle of $\widehat{\mathcal{E}}$ was first constructed in $[39, \S 7]$.

By [22, Theorem 3.7], we have the equation of currents on $\widehat{\mathcal{E}}$,

$$
d \psi=\tau^{*} e\left(\widehat{E}, \nabla^{\widehat{E}}\right)-\delta_{M}
$$

Moreover, $\psi$ is smooth on $\widehat{\mathcal{E}} \backslash\{0\}$, and the restriction of $-\psi$ to the fibres $\widehat{E}$ coincides with the solid angle form associated with the metric $g \widehat{E}$.

\subsection{The forms $a_{t}, b_{t}$ on $M$}

We use the same notation as in $\S 1.4$. In particular, we take $N$ as in that subsection, and we assume that $R \in \mathscr{A}^{U}$ is given by $(1.23)$.

We fix once and for all a square root $(2 i \pi)^{1 / 2}$ of $2 i \pi$. Our formulae will not depend on this choice.

Definition 1.10. Let $\varphi$ be the endomorphism of $\Lambda^{*}\left(T^{*} M\right) \otimes_{\mathbf{R}} \mathbf{C}$ which maps $\alpha \in$ $\Lambda^{k}\left(T^{*} M\right) \otimes_{\mathbf{R}} \mathbf{C}$ into $(2 i \pi)^{-k / 2} \alpha$.

Definition 1.11. Let $\alpha$ be the even form on $M$,

$$
\alpha=\varphi R\left(\theta^{\mathfrak{p}, 2}\right) .
$$


Proposition 1.12. The following identity holds:

$$
\alpha=R(0) \text {. }
$$

Proof. Using (1.40) and the fact that $R$ is Ad-invariant, for $s \in \mathbf{R}$, we get

$$
\begin{aligned}
\frac{\partial}{\partial s} \exp \left(s \theta^{\mathfrak{p}, 2}\right) R(0) & =\theta^{\mathfrak{p}, 2} \exp \left(s \theta^{\mathfrak{p}, 2}\right) R(0) \\
& =\frac{1}{2}\left(\operatorname{ad}\left(\theta^{\mathfrak{p}}\right)\left[\theta^{\mathfrak{p}} \exp \left(s \theta^{\mathfrak{p}, 2}\right)\right]\right) R(0)=0,
\end{aligned}
$$

from which we get (1.80).

Set

$$
\mathcal{N}=P_{K} \times_{K} N
$$

In (1.82), we can as well replace $K$ by $U, G, G_{\mathbf{C}}$. Then $q: \mathcal{N} \rightarrow M$ is a proper submersion with compact fibre $N$. Also, from the connection $\theta^{\mathfrak{k}}$ on $P_{K}$, we get a horizontal subbundle $T^{H} \mathcal{N} \subset T \mathcal{N}$. The closed 2-form $\eta$ on $N$ can be viewed as a vertical 2 -form on $\mathcal{N}$. By (1.19), $\mu$ can be viewed as a smooth map from $\mathcal{N}$ into $q^{*} \mathfrak{u}_{r}^{*}$. Let $\kappa$ be the 2 -form on $\mathcal{N}$,

$$
\kappa=\eta+\left\langle\mu, \theta^{\mathfrak{p}, 2}\right\rangle
$$

By $(1.20),(1.39)$, the 2 -form $\kappa$ is closed on $\mathcal{N}$.

We still define $\sigma_{\beta}$ as in $(1.25)$. Then $\sigma_{\beta}$ is a smooth section of $q^{*} \widehat{E}^{*}$ on $\mathcal{N}$.

Definition 1.13. We will say that $\beta$ is nondegenerate if $\sigma_{\beta}$ does not vanish on $\mathcal{N}$.

Definition 1.14. For $t \geqslant 0$, set

$$
\begin{aligned}
& a_{t}=(2 i \pi)^{m / 2} \varphi \int^{\widehat{B}} \exp \left(-\sigma\left(\mathcal{A}_{t}^{2}\right)\right) R(0), \\
& b_{t}=(2 i \pi)^{(m-1) / 2} \varphi \int^{\widehat{B}} \frac{\beta}{2 \sqrt{t}} \exp \left(-\sigma\left(\mathcal{A}_{t}^{2}\right)\right) R(0) .
\end{aligned}
$$

Then $a_{t}, b_{t}$ are smooth forms on $M$, and, $\bmod 2$, the degrees of $a_{t}, b_{t}$ are equal to $\operatorname{dim} \widehat{E}, \operatorname{dim} \widehat{E}-1$.

By (1.23), (1.47), we get

$$
a_{t}=\int^{\widehat{B}}\left[\exp \left(-\frac{1}{4}\left\langle\widehat{e}_{i}, R^{\widehat{E}} \widehat{e}_{j}\right) \widehat{e}^{i} \widehat{e}^{j}\right)\left(\exp \left(-t|\beta|^{2}\right) R\right)\left(\frac{\theta^{\mathfrak{p}, 2}}{2 i \pi}-\sqrt{t} \nabla^{\widehat{E} \otimes \mathfrak{g}_{r}, u} \beta-2 i \pi t \beta^{2}\right)\right] \text {. }
$$

A similar formula can be given for $b_{t}$. Therefore $a_{t}, b_{t}$ are real forms.

We identify $\widehat{E}$ and $\widehat{E}^{*}$ by the metric $g^{\widehat{E}}$, so that $\sigma_{\beta}$ is a section of $q^{*} \widehat{E}$ on $\mathcal{N}$. Then $\sigma_{\beta}^{*} \underline{a}_{t}, \sigma_{\beta}^{*} \underline{b}_{t}$ are smooth forms on $\mathcal{N}$. By $(1.74)$, we have the identities

$$
\begin{aligned}
\sigma_{\beta}^{*} \underline{a}_{t} & =\int^{\widehat{B}} \exp \left(-\frac{1}{4}\left\langle\widehat{e}_{i}, R^{\widehat{E}} \widehat{e}_{j}\right) \widehat{e}^{i} \widehat{e}^{j}-\nabla^{\widehat{E}} \sqrt{t} \sigma_{\beta}-t\left|\sigma_{\beta}\right|^{2}\right), \\
\sigma_{\beta}^{*} \underline{b}_{t} & =\int^{\widehat{B}} \frac{\sigma_{\beta}}{2 \sqrt{t}} \exp \left(-\frac{1}{4}\left\langle\widehat{e}_{i}, R^{\widehat{E}} \widehat{e}_{j}\right) \widehat{e}^{i} \widehat{e}^{j}-\nabla^{\widehat{E}} \sqrt{t} \sigma_{\beta}-t\left|\sigma_{\beta}\right|^{2}\right) .
\end{aligned}
$$

Moreover, $\sigma_{\beta}^{*} \underline{a}_{t}$ is a closed form. 
We will now extend these results to the forms $a_{t}, b_{t}$. Note that the connection $\nabla^{\widehat{E} \otimes \mathfrak{g}_{r}, u}$ on $\widehat{E} \otimes \mathfrak{g}_{r}$ lifts to $\mathcal{N}$.

If $\left.\alpha_{t}\right|_{t \geqslant 0}$ is a family of smooth forms on $M$, and if $K$ is a compact subset of $M$, given $c_{K}>0$, we will say that, on $K$, as $t \rightarrow+\infty, \alpha_{t}=\mathcal{O}\left(e^{-c_{K} t}\right)$ if the sup norm of $\alpha_{t}$ and of its derivatives of arbitrary order over $K$ are $\mathcal{O}\left(e^{-c_{K} t}\right)$.

Theorem 1.15. For any $t \geqslant 0$, the form $a_{t}$ is closed, and its cohomology class does not depend on $t \geqslant 0$. We have the identity

$$
\frac{\partial}{\partial t} a_{t}=-d b_{t}
$$

The following identities hold:

$$
\begin{aligned}
a_{t}=\int^{\widehat{B}} & {\left[\int _ { N } \operatorname { e x p } \left(-\frac{1}{4}\left\langle\widehat{e}_{i}, R^{\widehat{E}} \widehat{e}_{j}\right) \widehat{e}^{i} \widehat{e}^{j}\right.\right.} \\
+ & \left.\left.\left\langle\mu, \theta^{\mathfrak{p}, 2}-2 i \pi \sqrt{t} \nabla^{\widehat{E} \otimes \mathfrak{g}_{r}, u} \beta+4 \pi^{2} t \beta^{2}\right\rangle-4 \pi^{2} t|\langle\mu, i \beta\rangle|^{2}+\eta\right)\right], \\
b_{t}=\int^{\widehat{B}} & {\left[\int _ { N } \frac { 2 \pi \langle \mu , i \beta \rangle } { 2 \sqrt { t } } \operatorname { e x p } \left(-\frac{1}{4}\left\langle\widehat{e}_{i}, R^{\widehat{E}} \widehat{e}_{j}\right\rangle \widehat{e}^{i} \widehat{e}^{j}\right.\right.} \\
& \left.\left.+\left\langle\mu, \theta^{\mathfrak{p}, 2}-2 i \pi \sqrt{t} \nabla^{\widehat{E} \otimes \mathfrak{g}_{r}, u} \beta+4 \pi^{2} t \beta^{2}\right\rangle-4 \pi^{2} t|\langle\mu, i \beta\rangle|^{2}+\eta\right)\right] .
\end{aligned}
$$

Moreover,

$$
a_{t}=q_{*}\left[\sigma_{\beta}^{*} \underline{a}_{t} \exp (\kappa)\right], \quad b_{t}=q_{*}\left[\sigma_{\beta}^{*} \underline{b}_{t} \exp (\kappa)\right] .
$$

Also,

$$
a_{0}=e\left(\widehat{E}, \nabla^{\widehat{E}}\right) \varphi R\left(\theta^{\mathfrak{p}, 2}\right)=e\left(\widehat{E}, \nabla^{\widehat{E}}\right) q_{*}[\exp (\kappa)]=e\left(\widehat{E}, \nabla^{\widehat{E}}\right) R(0) .
$$

If $\beta$ is nondegenerate, for any compact set $K \subset M$, there exists $c_{K}>0$ such that, on $K$, as $t \rightarrow+\infty$,

$$
a_{t}=\mathcal{O}\left(e^{-c_{K} t}\right), \quad b_{t}=\mathcal{O}\left(e^{-c_{K} t}\right) .
$$

If $\beta$ is nondegenerate, the Euler class $e(\widehat{E})$ vanishes. Also, $\sigma_{\beta}^{*} \psi$ is a smooth $(m-1)$-form on $\mathcal{N}$ with values in $o(\widehat{E})$ such that

$$
d\left(\sigma_{\beta}^{*} \psi\right)=q^{*} e\left(\widehat{E}, \nabla^{\widehat{E}}\right)
$$

Proof. The operator $\nabla .{ }^{\widehat{E} \otimes \mathfrak{g}_{r}, u}+2 i_{\sqrt{t} \beta}+\operatorname{ad}(\sqrt{t} \beta)$ is a derivation acting on the smooth sections of $\Lambda^{\cdot}\left(T^{*} M\right) \widehat{\otimes} \Lambda^{*}\left(\widehat{E}^{*}\right) \otimes S \mathfrak{g}_{r}$. By the first identity in (1.62), we get

$$
\left(\nabla^{\widehat{E} \otimes \mathfrak{g}_{r}, u}+2 i_{\sqrt{t} \beta}+\operatorname{ad}(\sqrt{t} \beta)\right) \exp \left(-\sigma\left(\mathcal{A}_{t}^{2}\right)\right)=0 .
$$

Clearly,

$$
\int^{\widehat{B}} i_{\beta} \exp \left(-\sigma\left(\mathcal{A}_{t}^{2}\right)\right) R(0)=0 .
$$


Moreover, since $R$ is Ad-invariant, we get

$$
\int^{\widehat{B}} \operatorname{ad}(\beta) \exp \left(-\sigma\left(\mathcal{A}_{t}^{2}\right)\right) R(0)=0 .
$$

By (1.84), (1.94), and (1.95), we get

$$
d a_{t}=0
$$

Since $a_{t}$ is obtained from $a_{1}$ by replacing $\beta$ by $\sqrt{t} \beta$, by the universality of the construction of $a_{t}$, the cohomology class of $a_{t}$ does not depend on $t$. By the second identity in (1.62), we get (1.87). Another proof is to observe that, by (1.68), the analogue $\mathbf{a}_{1}$ of $a_{1}$ on $M \times \mathbf{R}_{+}^{*}$ is given by

$$
\mathbf{a}_{1}=a_{t}-d t \wedge b_{t}
$$

Since $\mathbf{a}_{1}$ is closed, from (1.97), we get (1.87).

By (1.47), we get

$$
\begin{aligned}
\sigma\left(\mathcal{A}_{t}^{2}\right) \exp (2 i \pi\langle\mu, A\rangle)= & \left(\frac{1}{4}\left\langle\widehat{e}_{i}, R^{\widehat{E}} \widehat{e}_{j}\right\rangle \widehat{e}^{i} \widehat{e}^{j}\right. \\
& \left.+2 i \pi\left\langle\mu,-\theta^{\mathfrak{p}, 2}+\sqrt{t} \nabla^{\widehat{E} \otimes \mathfrak{g}_{r}, u} \beta+t \beta^{2}\right\rangle+4 \pi^{2} t|\langle\mu, i \beta\rangle|^{2}\right) \\
& \times \exp (2 i \pi\langle\mu, A\rangle) .
\end{aligned}
$$

By (1.23), (1.84), and (1.98), we get (1.88).

Clearly, $\nabla^{\widehat{E}} \sigma_{\beta}$ is a 1 -form on $\mathcal{N}$. This form can be split into its horizontal and vertical components. Let $d^{V} \mu$ denote the fibrewise derivative of $\mu$. By (1.25), we get

$$
\nabla^{\widehat{E}} \sigma_{\beta}=2 \pi\left\langle\mu, \nabla^{\widehat{E} \otimes \mathfrak{g}_{r}, u} i \beta\right\rangle+2 \pi\left\langle d^{V} \mu, i \beta\right\rangle .
$$

By (1.20), (1.99), and checking signs carefully, we obtain

$$
\nabla^{\widehat{E}} \sigma_{\beta}=2 \pi\left\langle\mu, \nabla^{\widehat{E} \otimes \mathfrak{g}_{r}, u} i \beta\right\rangle-2 \pi i_{(i \beta)^{N}} \eta
$$

Now, we evaluate the integral $q_{*}\left[\sigma_{\beta}^{*} \underline{a}_{t} \exp (\kappa)\right]$ using (1.86). By (1.83), (1.100), the only nonhorizontal form that appears in the exponential is just $\eta+2 \pi \sqrt{t} i_{(i \beta)^{N}} \eta$. Using an elementary reduction of squares, in the fibre integral, the form $\eta+2 \pi \sqrt{t} i_{(i \beta)^{N}} \eta$ can be replaced by $\eta-4 \pi^{2} \frac{t}{2} \eta\left((i \beta)^{N},(i \beta)^{N}\right)$. By $(1.21)$,

$$
\eta\left((i \beta)^{N},(i \beta)^{N}\right)=-2\left\langle\mu, \beta^{2}\right\rangle .
$$

By (1.101), in (1.89), we can replace $\eta+2 \pi \sqrt{t} i_{(i \beta)^{N}} \eta$ by $\eta+\left\langle\mu, 4 \pi^{2} t \beta^{2}\right\rangle$. Therefore, the right-hand sides of (1.88) and (1.89) coincide.

By (1.30), (1.79), (1.80), (1.85), (1.86), and (1.89), we get (1.90).

If the section $\sigma_{\beta}$ does not vanish, by $(1.24)-(1.27)$, and by $(1.85),(1.86)$, and (1.88) or (1.89), we get (1.91). Therefore the common cohomology class of the $a_{t}$ vanishes identically. Since $R(0) \neq 0$, by $(1.90)$, the class $e(\widehat{E})$ vanishes. By the results of $\S 1.11$, and in particular by (1.78), we get the last statement of our theorem, whose proof is completed.

Remark 1.16. Our results on the forms $a_{t}, b_{t}$ also follow from (1.75), (1.89). 


\section{The $W$-invariant}

Let $\pi: M \rightarrow S$ be a projection of smooth manifolds with compact fibre $X$. We assume that $M$ is equipped with the same geometric data as in $\S 1$, with $\widehat{E}$ being $\widehat{T X}$, another copy of the relative tangent bundle $T X$. The purpose of this section is to construct odd closed forms $\pi_{*} c_{t}$ on the base $S$, and by transgression an even form $\pi_{*} W$ on $S$. Also, we prove the compatibility of the integral $\pi_{*} W$ with respect to natural functorial operations, which make it to behave in a way that is similar to the analytic torsion forms of Bismut and Lott [18].

This section is organized as follows. In $\S 2.1$, we introduce the smooth fibration $\pi$ : $M \rightarrow S$.

In $\S 2.2$, we define various tensors associated with the fibration.

In $\S 2.3$, in the context of $\S 1$, we take $\widehat{E}=\widehat{T X}, \beta=\widehat{\theta}^{\mathfrak{p}}$.

In $\S 2.4$, we exchange the roles of the exterior variables in $\Lambda^{*}\left(T^{*} X\right)$ and $\Lambda^{*}\left(\widehat{T^{*} X}\right)$, so as to get refined Bianchi-like identities on $\sigma\left(\mathcal{A}_{t}^{2}\right)$. We show that $\pi_{*} a_{t}$ is concentrated in degree 0 , and that $\pi_{*} b_{t}$ vanishes identically.

In $\S 2.5$, we construct forms $c_{t}$ on $M$ such that $\pi_{*} c_{t}$ is an odd closed form whose cohomology class does not depend on $t$.

In $\S 2.6$, by transgression of the forms $\pi_{*} c_{t}$, if $\widehat{\theta}^{\mathfrak{p}}$ is nondegenerate, we obtain an even form $\pi_{*} W$ on $S$, which is closed if the fibres $X$ are odd dimensional.

In $\S 2.7$, we study the behaviour of $\pi_{*} W$ under adiabatic limits.

Finally, in $\S 2.8$, we study the rigidity of certain cohomology classes under deformation of the flat connection on $P_{G}$.

\subsection{A smooth fibration}

Let $\pi: M \rightarrow S$ be a submersion of smooth manifolds with compact fibre $X$ of dimension $m$. Let $r$ be the dimension of $S$. Let $T X \subset T M$ be the tangent bundle to the fibres $X$.

Let $T^{H} M \subset T M$ be a horizontal subbundle, so that

$$
T M=T^{H} M \oplus T X .
$$

Let $P^{T X}: T M \rightarrow T X$ be the projection associated to the splitting (2.1). Observe that

$$
T^{H} M \simeq \pi^{*} T S
$$

By (2.1), (2.2), we have the identification of bundles of algebras

$$
\Lambda^{*}\left(T^{*} M\right) \simeq \pi^{*} \Lambda^{*}\left(T^{*} S\right) \widehat{\otimes} \Lambda^{*}\left(T^{*} X\right) .
$$

If $U \in T S$, let $U^{H} \in T^{H} M$ be the horizontal lift of $U$, so that $\pi_{*} U^{H}=U$.

Definition 2.1. If $U, V$ are smooth sections of $T S$, set

$$
T^{H}(U, V)=-P^{T X}\left[U^{H}, V^{H}\right] .
$$

\subsection{A metric on $T X$ and the tensors $T$ and $S$}

Let $g^{T X}$ be an Euclidean metric on $T X$. In what follows, we identify $T X$ and $T^{*} X$ by the metric $g^{T X}$. 
By $[8, \S 1],\left(T^{H} M, g^{T X}\right)$ determines an Euclidean connection $\nabla^{T X}$ on $T X$. Let $g^{T S}$ be a Euclidean metric on $T S$. We equip $T M$ with the metric $g^{T M}=\pi^{*} g^{T S} \oplus g^{T X}$. Let $\nabla^{T M, L}$ be the Levi-Civita connection on $\left(T M, g^{T M}\right)$. Then $\nabla^{T X}$ is given by

$$
\nabla^{T X}=P^{T X} \nabla^{T M, L} .
$$

Note that $\nabla^{T X}$ does not depend on $g^{T S}$.

Let $\nabla^{T S}$ be the Levi-Civita connection on $\left(T S, g^{T S}\right)$. Let $\nabla^{T M}$ be the connection on $T M$ given by

$$
\nabla^{T M}=\pi^{*} \nabla^{T S} \oplus \nabla^{T X}
$$

Let $T$ be the torsion of $\nabla^{T M}$. Put

$$
S=\nabla^{T M, L}-\nabla^{T M} .
$$

Then $S$ is a 1 -form on $M$ with values in antisymmetric elements of $\operatorname{End}(T M)$. If $A, B, C \in$ $T M$, then

$$
\begin{aligned}
& S(A) B-S(B) A+T(A, B)=0, \\
& 2\langle S(A) B, C\rangle+\langle T(A, B), C\rangle+\langle T(C, A), B\rangle-\langle T(B, C), A\rangle=0 .
\end{aligned}
$$

By [8, Theorem 1.9], we know that the following hold.

- The connection $\nabla^{T X}$ and the tensors $T$ and $\langle S(\cdot) \cdot, \cdot\rangle$ do not depend on $g^{T S}$.

- The tensor $T$ takes its values in $T X$, and vanishes on $T X \times T X$.

- For any $A \in T M, S(A)$ maps $T X$ into $T^{H} M$.

- For any $A, B \in T^{H} M, S(A) B \in T X$.

- If $A \in T^{H} M, S(A) A=0$.

By (2.8), we find that if $A \in T^{H} M, B, C \in T X$,

$$
\langle T(A, B), C\rangle=\langle T(A, C), B\rangle=-\langle S(B) C, A\rangle .
$$

If $U$ is a smooth section of $T S$, let $L_{U^{H}}$ denote the Lie derivative associated with the vector field $U^{H}$ on $M$. Then $L_{U^{H}}$ acts on the tensor algebra of $T X$, and this action is tensorial in $U \in T S$. In particular, $\left(g^{T X}\right)^{-1} L_{U^{H}} g^{T X}$ is a self-adjoint section of $\operatorname{End}(T X)$.

Now, we recall a simple result stated in [9, Theorem 1.1].

Theorem 2.2. The connection $\nabla^{T X}$ on $\left(T X, g^{T X}\right)$ is characterized by the following two properties.

- Along the fibres $X, \nabla^{T X}$ is just the Levi-Civita connection of $\left(T X, g^{T X}\right)$.

- If $U \in T S$, then

$$
\nabla_{U^{H}}^{T X}=L_{U^{H}}+\frac{1}{2}\left(g^{T X}\right)^{-1} L_{U^{H}} g^{T X}
$$

If $U, V \in T S$, then

$$
T\left(U^{H}, V^{H}\right)=T^{H}(U, V)
$$

If $U \in T S, A \in T X$, then

$$
T\left(U^{H}, A\right)=\frac{1}{2}\left(g^{T X}\right)^{-1} L_{U^{H}} g^{T X} A .
$$


We denote by $R^{T X}$ the curvature of $\nabla^{T X}$.

Let $d v_{X}$ be the volume form along $X$. If $U \in T S$, let $\operatorname{div}_{X}(U)$ be defined by

$$
L_{U^{H}} d v_{X}=\operatorname{div}_{X}(U) d v_{X} .
$$

By [13, Proposition 1.4] or by $(2.9),(2.12)$, if $e_{1}, \ldots, e_{m}$ is an orthonormal basis of $T X$, then

$$
\operatorname{div}_{X}(U)=-\left\langle S\left(e_{i}\right) e_{i}, U^{H}\right\rangle .
$$

\subsection{The case where $\widehat{E}=\widehat{T X}, \beta=\widehat{\theta}^{\mathfrak{p}}$}

We make the same assumptions as in $\S \S 2.1$ and 2.2. Also, we assume that $G$ is a reductive group as in $\S 1.1$, and that the formalism developed in $\S \S 1.1-1.6$ applies to our manifold $M$. We will use the notation of these subsections without further mention.

Recall that $\mathcal{S}$ denotes the manifold of smooth sections $h$ of $P_{G} \times{ }_{G} G / K$. Let $\mathcal{S}^{\prime}$ denote the manifold of smooth choices $\left(h, T^{H} M, g^{T X}\right)$. In what follows, we will often replace $M, S$ by $M \times \mathcal{S}^{\prime}, S \times \mathcal{S}^{\prime}$.

In what follows, we fix $h \in \mathcal{S}$, so that the principal $G$-bundle $p: P_{G} \rightarrow M$ can be reduced to a principal $K$-bundle $p: P_{K} \rightarrow M$.

Let $\widehat{T X}$ be another copy of $T X$. Let $g^{\widehat{T X}}$ be the metric on $\widehat{T X}$ which corresponds to $g^{T X}$, and let $\nabla^{\widehat{T X}}$ be the Euclidean connection corresponding to $\nabla^{T X}$. We will use the formalism of $\S 1.7$ with $\left(\widehat{E}, g^{\widehat{E}}, \nabla^{\widehat{E}}\right)=\left(\widehat{T X}, g^{T X}, \nabla^{\widehat{T X}}\right)$. We denote by $\int^{\widehat{B}}$ the Berezin integral of $\S 1.5$, which maps $\Lambda^{\cdot}\left(T^{*} M\right) \widehat{\otimes} \Lambda^{\cdot}\left(\widehat{T^{*} X}\right)$ into $\Lambda^{*}\left(T^{*} M\right) \otimes o(T X)$.

Let $e_{1}, \ldots, e_{m}$ be an orthonormal basis of $T X$, and let $\widehat{e}_{1}, \ldots, \widehat{e}_{m}$ be the corresponding orthonormal basis of $\widehat{T X}$. Let $f_{1}, \ldots, f_{r}$ be a basis of $T S$. The associated dual bases will be denoted with upper indices.

Recall that $\theta^{\mathfrak{p}}$ is a smooth section of $T^{*} M \otimes \mathfrak{p}_{r}$. Set

$$
\theta^{\mathfrak{p}, X}=\sum_{i=1}^{m} e^{i} \theta^{\mathfrak{p}}\left(e_{i}\right), \quad \theta^{\mathfrak{p}, H}=\sum_{\alpha=1}^{r} f^{\alpha} \theta^{\mathfrak{p}}\left(f_{\alpha}^{H}\right),
$$

so that

$$
\theta^{\mathfrak{p}}=\theta^{\mathfrak{p}, X}+\theta^{\mathfrak{p}, H}
$$

Set

$$
\widehat{\theta}^{\mathfrak{p}}=\sum_{i=1}^{m} \widehat{e}^{i} \theta^{\mathfrak{p}}\left(e_{i}\right)
$$

In the context of $\S 1.7$, we take

$$
\beta=\widehat{\theta}^{\mathfrak{p}} \text {. }
$$

We can now use the notation and results of $\S 1$, while replacing $\beta$ by $\widehat{\theta}^{\mathfrak{p}}$.

By (1.47), (2.18), we get

$$
\sigma\left(\mathcal{A}_{t}^{2}\right)=\frac{1}{4}\left\langle e_{i}, R^{T X} e_{j}\right\rangle \widehat{e}^{i} \widehat{e}^{j}-\theta^{\mathfrak{p}, 2}+\nabla^{\widehat{T X} \otimes \mathfrak{g}_{r}, u} \sqrt{t} \widehat{\theta}^{\mathfrak{p}}+t\left|\widehat{\theta}^{\mathfrak{p}}\right|^{2}+t \widehat{\theta}^{\mathfrak{p}, 2} .
$$

By (1.61), the operator $\mathcal{L}_{t}$ is given by

$$
\mathcal{L}_{t}=\nabla^{\widehat{T X} \otimes \mathfrak{g}_{r}, u}+2 i_{\sqrt{t} \widehat{\theta}^{\mathfrak{p}}}+\operatorname{ad}\left(\sqrt{t} \widehat{\theta}^{\mathfrak{p}}\right) .
$$


By (1.62), (2.18), we get

$$
\mathcal{L}_{t} \sigma\left(\mathcal{A}_{t}^{2}\right)=0, \quad \frac{\partial}{\partial t} \sigma\left(\mathcal{A}_{t}^{2}\right)=\mathcal{L}_{t} \frac{\widehat{\theta}^{\mathfrak{p}}}{2 \sqrt{t}} .
$$

\subsection{Exchanging the hatted and unhatted variables}

We will exchange the roles of $\Lambda^{\cdot}\left(T^{*} X\right)$ and $\Lambda^{\cdot}\left(\widehat{T^{*} X}\right)$. In particular, $\Lambda^{*}\left(\widehat{T^{*} X}\right)$ will be considered as the standard exterior algebra of the cotangent bundle $\widehat{T^{*} X}$ of the fibre $X$.

We denote by $\widehat{d}^{X}$ the fibrewise de Rham operator acting on smooth sections of $\Lambda^{*}\left(\widehat{T^{*} X}\right)$. Also, $\pi^{*} \Lambda^{*}\left(T^{*} S\right)$ is treated as a fibrewise trivial vector bundle, so that $\widehat{d}^{X}$ also acts on smooth sections of $\pi^{*} \Lambda^{*}\left(T^{*} S\right) \widehat{\otimes} \Lambda^{*}\left(\widehat{T^{*} X}\right)$.

Let $\widehat{\nabla}^{T X}$ be the restriction of $\nabla^{T X}$ to a given fibre. Then $\widehat{\nabla}^{T X}$ is a fibrewise connection on $T X$. Its curvature, $\widehat{R}^{T X}$, is a smooth section of $\Lambda^{2}\left(\widehat{T^{*} X}\right) \otimes \operatorname{End}(T X)$. The connection $\widehat{\nabla}^{T X}$ acts on smooth fibrewise sections of $\Lambda^{\cdot}\left(\widehat{T^{*} X}\right) \widehat{\otimes} \Lambda^{*}\left(T^{*} X\right)$. Its action on sections of $\Lambda \cdot\left(\widehat{T^{*} X}\right)$ is just given by the de Rham operator $\widehat{d}^{X}$. Of course, $\widehat{\nabla}^{T X}$ also acts on smooth sections of $\pi^{*} \Lambda^{*}\left(T^{*} S\right) \widehat{\otimes} \Lambda^{*}\left(\widehat{T^{*} X}\right) \widehat{\otimes} \Lambda^{*}\left(T^{*} X\right)$.

As in $[16$, equation $(3.61)]$, set

$$
\begin{aligned}
\widehat{T}^{H} & =\frac{1}{2}\left\langle T\left(f_{\alpha}^{H}, f_{\beta}^{H}\right), e_{i}\right) \widehat{e}^{i} \wedge f^{\alpha} \wedge f^{\beta}, \\
T^{0} & =f^{\alpha} \wedge \widehat{e}^{i} \wedge T\left(f_{\alpha}^{H}, e_{i}\right) .
\end{aligned}
$$

Then $\widehat{T}^{H}$ and $\widehat{d}^{X} \widehat{T}^{H}$ are sections of $\pi^{*} \Lambda^{2}\left(T^{*} S\right) \widehat{\otimes} \Lambda^{\cdot}\left(\widehat{T^{*} X}\right)$. Moreover, $T^{0}$ is a section of $\pi^{*} \Lambda^{*}\left(T^{*} S\right) \widehat{\otimes} \Lambda^{*}\left(\widehat{T^{*} X}\right) \widehat{\otimes} \Lambda^{*}(T X)$. We will view $T^{0}$ as a section of $\pi^{*} \Lambda^{*}\left(T^{*} S\right) \widehat{\otimes} \Lambda^{*}\left(\widehat{T^{*} X}\right)$ with values in $T X$. The operator $i_{T^{0}}$ decreases by 1 the degree in $\Lambda^{*}\left(T^{*} X\right)$, and increases by 2 the degree in $\pi^{*} \Lambda^{*}\left(T^{*} S\right) \widehat{\otimes} \Lambda^{\cdot}\left(\widehat{T^{*} X}\right)$.

As in $[16$, equation $(3.63)]$, set

$$
\left|T^{0}\right|^{2}=\left\langle T^{0}, T^{0}\right\rangle
$$

Then

$$
\left|T^{0}\right|^{2}=\sum_{j=1}^{m}\left(\sum_{\substack{1 \leqslant i \leqslant m \\ 1 \leqslant \alpha \leqslant r}}\left\langle T\left(f_{\alpha}^{H}, e_{i}\right), e_{j}\right) f^{\alpha} \wedge \widehat{e}^{i}\right)^{2}
$$

where the square in the right-hand side of $(2.24)$ is taken in $\Lambda^{*}\left(T^{*} S\right) \widehat{\otimes} \Lambda^{*}\left(\widehat{T^{*} X}\right)$. Then $\left|T^{0}\right|^{2}$ is a section of $\Lambda^{2}\left(T^{*} S\right) \widehat{\otimes} \Lambda^{2}\left(\widehat{T^{*} X}\right)$.

When identifying $T X$ and $T^{*} X$ by the metric $g^{T X}, T^{0}$ can be viewed as a section of $\pi^{*} \Lambda^{*}\left(T^{*} S\right) \widehat{\otimes} \Lambda^{\cdot}\left(\widehat{T^{*} X}\right) \widehat{\otimes} \Lambda^{\cdot}\left(T^{*} X\right)$, which is given by

$$
T^{0}=\left\langle T\left(f_{\alpha}^{H}, e_{i}\right), e_{j}\right\rangle f^{\alpha} \wedge \widehat{e}^{i} \wedge e^{j}
$$

By the above, $\widehat{\nabla}^{T X} T^{0}$ is well defined.

By [16, Theorem 3.26], we get

$$
\frac{1}{2}\left\langle e_{i},\left.R^{T X} e_{j}\left|\widehat{e}^{i} \wedge \widehat{e}^{j}=\frac{1}{2}\left\langle e_{i}, \widehat{R}^{T X} e_{j}\right\rangle e^{i} \wedge e^{j}+\widehat{\nabla}^{T X} T^{0}+\frac{1}{2}\right| T^{0}\right|^{2}-\frac{1}{2} \widehat{d}^{X} \widehat{T}^{H} .\right.
$$

If the metric $g^{T X}$ is replaced by $g^{T X} / t$, equation (2.26) is divided by $t$. 
Let $N^{\Lambda^{*}\left(T^{*} M\right)}, N^{\Lambda^{*}\left(\widehat{T^{*} X}\right)}$ be the number operators of $\Lambda^{\cdot}\left(T^{*} M\right), \Lambda^{*}\left(\widehat{T^{*} X}\right)$ that act by multiplication by the corresponding degrees. Set

$$
\mathcal{N}^{d}=N^{\Lambda^{\cdot}\left(T^{*} M\right)}-N^{\Lambda^{\cdot}\left(\widehat{T^{*} X}\right)}
$$

Definition 2.3. Let $\widehat{\mathcal{L}_{t}}$ be the fibrewise differential operator

$$
\widehat{\mathcal{L}}_{t}=\widehat{\nabla}^{T X \otimes \mathfrak{g}_{r}, u}+2 i_{-\sqrt{t} \theta^{\mathfrak{p}, X}+\frac{1}{2} T^{0}}+\frac{\operatorname{ad}\left(\theta^{\mathfrak{p}}\right)}{\sqrt{t}} .
$$

Theorem 2.4. The following identity holds:

$$
\begin{aligned}
\sigma\left(\mathcal{A}_{t}^{2}\right)= & \frac{1}{4}\left\langle e_{i}, \widehat{R}^{T X} e_{j}\right\rangle e^{i} e^{j}+\widehat{\nabla}^{T X \otimes \mathfrak{g}_{r}, u}\left(-\sqrt{t} \theta^{\mathfrak{p}, X}+\frac{1}{2} T^{0}\right) \\
& +\left|-\sqrt{t} \theta^{\mathfrak{p}, X}+\frac{1}{2} T^{0}\right|^{2}+t \widehat{\theta}^{\mathfrak{p}, 2}-\theta^{\mathfrak{p}, 2}-\widehat{\nabla}^{T X \otimes \mathfrak{g}_{r}, u} \sqrt{t} \theta^{\mathfrak{p}, H}-\frac{1}{4} \widehat{d}^{X} \widehat{T}^{H} .
\end{aligned}
$$

Moreover, for $t>0$, we have the identities

$$
\begin{aligned}
& \widehat{\mathcal{L}}_{t} \sigma\left(\mathcal{A}_{t}^{2}\right)=0, \\
& \frac{\partial}{\partial t} \sigma\left(\mathcal{A}_{t}^{2}\right)=-\widehat{\mathcal{L}}_{t} \frac{\theta^{\mathfrak{p}}}{2 \sqrt{t}}+\widehat{\theta}^{\mathfrak{p}, 2}+\frac{\theta^{\mathfrak{p}, 2}}{t}, \\
& \frac{\partial}{\partial t} \sigma\left(\mathcal{A}_{t}^{2}\right)+\frac{1}{2 t}\left[\mathcal{N}^{d}, \sigma\left(\mathcal{A}_{t}^{2}\right)\right]=-\widehat{\mathcal{L}}_{t} \frac{\theta^{\mathfrak{p}}}{2 \sqrt{t}} .
\end{aligned}
$$

Finally, the following identities hold:

$$
\begin{aligned}
& \mathcal{L}_{t} \theta^{\mathfrak{p}}=t \widehat{\mathcal{L}}_{t} \widehat{\theta}^{\mathfrak{p}}=\sqrt{t}\left[\widehat{\theta}^{\mathfrak{p}}, \theta^{\mathfrak{p}}\right] \\
& \mathcal{L}_{t} \widehat{\theta}^{\mathfrak{p}}+\widehat{\mathcal{L}}_{t} \theta^{\mathfrak{p}}=2\left(\sqrt{t} \widehat{\theta}^{\mathfrak{p}, 2}+\frac{\theta^{\mathfrak{p}, 2}}{\sqrt{t}}\right) .
\end{aligned}
$$

Proof. We use the notation of $\S 2.2$. Let $\nabla^{T M}$ be the connection on $T M$ that was defined in (2.6). We still denote by $\nabla^{T M}$ its action on smooth sections of $\Lambda^{*}\left(T^{*} M\right)$, which increases the total degree by 1 . Since $T$ is the torsion of $\nabla^{T M}$, we have the well-known identity,

$$
d^{M}=\nabla^{T M}+i_{T}
$$

Let $\nabla^{\Lambda^{*}\left(T^{*} M\right) \otimes \mathfrak{g}_{r}, u}$ be the connection on $\Lambda^{\cdot}\left(T^{*} M\right) \otimes \mathfrak{g}_{r}$ which is induced by the connections $\nabla^{T M}, \nabla^{\mathfrak{g}_{r}, u}$. By (1.41), (2.32), we get

$$
\left(\nabla^{\Lambda^{\cdot}\left(T^{*} M\right) \otimes \mathfrak{g}_{r}, u}+i_{T}\right) \theta^{\mathfrak{p}}=0
$$

By (2.16), (2.33), we get

$$
\nabla^{\widehat{T X} \otimes \mathfrak{g}_{r}, u \widehat{\theta}^{\mathfrak{p}}}=-\left(\widehat{\nabla}^{T X \otimes \mathfrak{g}_{r}, u}+i_{T^{0}}\right) \theta^{\mathfrak{p}, X}-\widehat{\nabla}^{T X \otimes \mathfrak{g}_{r}, u} \theta^{\mathfrak{p}, H} .
$$

By (2.19), (2.26), and (2.34), we get (2.29). 
Now, we establish (2.30). By (1.39), (1.73), we get

$$
\begin{aligned}
& \left(\widehat{\nabla}^{T X \otimes \mathfrak{g}_{r}, u}+2 i_{-\sqrt{t} \theta^{\mathfrak{p}, X}+\frac{1}{2} T^{0}}\right)\left(\frac{1}{4}\left\langle e_{i}, \widehat{R}^{T X} e_{j}\right) e^{i} e^{j}\right. \\
& \left.+\widehat{\nabla}^{T X \otimes \mathfrak{g}_{r}, u}\left(-\sqrt{t} \theta^{\mathfrak{p}, X}+\frac{1}{2} T^{0}\right)+\left|-\sqrt{t} \theta^{\mathfrak{p}, X}+\frac{1}{2} T^{0}\right|^{2}\right)=\left[\widehat{\theta}^{\mathfrak{p}, 2}, \sqrt{t} \theta^{\mathfrak{p}, X}\right] .
\end{aligned}
$$

By (1.41), we obtain

$$
\widehat{\nabla}^{T X \otimes \mathfrak{g}_{r}, u} \widehat{\theta}^{\mathfrak{p}}=0
$$

Moreover,

$$
\widehat{\nabla}^{T X \otimes \mathfrak{g}_{r}, u} \theta^{\mathfrak{p}, 2}=\left[\widehat{\nabla}^{T X \otimes \mathfrak{g}_{r}, u} \theta^{\mathfrak{p}}, \theta^{\mathfrak{p}}\right] .
$$

Also, we have the easy identity

$$
-2 i_{-\sqrt{t} \theta^{\mathfrak{p}, X}+\frac{1}{2} T_{0}} \theta^{\mathfrak{p}, 2}+\left[\frac{\theta^{\mathfrak{p}}}{\sqrt{t}},\left|-\sqrt{t} \theta^{\mathfrak{p}, X}+\frac{1}{2} T^{0}\right|^{2}\right]=0 .
$$

By (2.29), and (2.34)-(2.38), we get the first identity in (2.30).

By (2.29), we obtain

$$
\frac{\partial}{\partial t} \sigma\left(\mathcal{A}_{t}^{2}\right)=-\frac{1}{2 \sqrt{t}} \widehat{\nabla}^{T X \otimes \mathfrak{g}_{r}, u} \theta^{\mathfrak{p}}-\frac{1}{\sqrt{t}} i_{-\sqrt{t} \theta^{\mathfrak{p}, X}+\frac{1}{2} T^{0}} \theta^{\mathfrak{p}}+\widehat{\theta}^{\mathfrak{p}, 2},
$$

which is equivalent to the second identity in (2.30). The third identity in (2.30) follows from (2.19) and from the second identity in (2.30).

By (1.41), (2.20), we get one identity in the first line of (2.31). By (2.28), (2.36), we obtain the second identity. By (2.21), (2.30), we get the last identity in (2.31). The proof of our theorem is completed.

Let $\chi(X)$ be the Euler characteristic of the fibre $X$. By the Chern-Gauss-Bonnet theorem, we get

$$
\chi(X)=\int_{X} e(T X) .
$$

If $m$ is odd, $\chi(X)=0$, and $e(T X)=0$.

Let $R \in \mathscr{A}^{U}$ be the function given by (1.23). Recall that, for $a \in \mathbf{R}$, the action of $\psi_{a}$ on $\Lambda^{\cdot}\left(T^{*} M\right)$ was defined in (1.51). We use the same notation for the corresponding action on $\Lambda^{*}\left(T^{*} S\right)$.

Theorem 2.5. For any $t \geqslant 0$, the following identities hold:

$$
\pi_{*} a_{t}=\chi(X) R(0), \quad \pi_{*} b_{t}=0 .
$$

Proof. To establish (2.41), we may as well replace $2 i \pi$ by 1 in equation (1.84) for $a_{t}, b_{t}$. Let $\widehat{\int_{X}}$ denote the standard integral along $X$, in which the exterior algebra is now generated by $\widehat{e}^{1}, \ldots, \widehat{e}^{m}$. Let $\int^{B}$ denote Berezin integration with respect to the variables $e^{1}, \ldots, e^{m}$. By (1.84), we get

$$
\pi_{*} a_{t}=\pi_{*} \int^{\widehat{B}} \exp \left(-\sigma\left(\mathcal{A}_{t}^{2}\right)\right) R(0)=(-1)^{m} \int_{X} \int^{B} \exp \left(-\sigma\left(\mathcal{A}_{t}^{2}\right)\right) R(0) .
$$

The sign $(-1)^{m}$ comes from the interchange between the two kinds of integral. 
Set

$$
\mathcal{R}_{t}=t^{\mathcal{N}^{d} / 2} \sigma\left(\mathcal{A}_{t}^{2}\right) t^{-\mathcal{N}^{d} / 2} .
$$

Using (2.42) and the fact that $T X$ and $\widehat{T X}$ have the same dimension, we get

$$
\psi_{\sqrt{t}} \pi_{*} a_{t}=(-1)^{m} \int_{X} \int^{B} \exp \left(-\mathcal{R}_{t}\right) R(0) .
$$

By the third equation in (2.30), and by (2.43), we obtain

$$
\frac{\partial}{\partial t} \mathcal{R}_{t}=-t^{\mathcal{N}^{d} / 2}\left(\widehat{\mathcal{L}}_{t} \frac{\theta^{\mathfrak{p}}}{2 \sqrt{t}}\right) \sigma\left(\mathcal{A}_{t}^{2}\right) t^{-\mathcal{N}^{d} / 2} .
$$

By (2.44), (2.45), for $t>0$, we get

$$
\frac{\partial}{\partial t} \psi_{\sqrt{t}} \pi_{*} a_{t}=(-1)^{m} \psi_{\sqrt{t}} \widehat{\int_{X}} \int^{B}\left(\widehat{\mathcal{L}}_{t} \frac{\theta^{\mathfrak{p}}}{2 \sqrt{t}}\right) \exp \left(-\sigma\left(\mathcal{A}_{t}^{2}\right)\right) R(0) .
$$

Using (2.30), we can rewrite (2.46) in the form

$$
\frac{\partial}{\partial t} \psi_{\sqrt{t}} \pi_{*} a_{t}=(-1)^{m} \psi_{\sqrt{t}} \int_{X} \int^{B} \widehat{\mathcal{L}}_{t}\left[\frac{\theta^{\mathfrak{p}}}{2 \sqrt{t}} \exp \left(-\sigma\left(\mathcal{A}_{t}^{2}\right)\right)\right] R(0) .
$$

Since $R$ is Ad-invariant, by (2.47), we get

$$
\frac{\partial}{\partial t} \psi_{\sqrt{t}} \pi_{*} a_{t}=0
$$

By (2.48), we deduce that, in positive degree, $\pi_{*} a_{t}$ vanishes, and that

$$
\left[\pi_{*} a_{t}\right]^{(0)}=\left[\pi_{*} a_{0}\right]^{(0)} .
$$

By (1.90), (2.40), we get

$$
\left[\pi_{*} a_{0}\right]^{(0)}=\pi_{*}\left[e\left(\widehat{T X}, \nabla^{\widehat{T X}}\right)\right] R(0)=\chi(X) R(0) .
$$

By the above, we get the first equation in (2.41).

We use the notation in the proof of Theorem 1.15. Then $\pi_{*} \mathbf{a}_{1}$ vanishes in positive degree. By (1.97), this implies the vanishing of $\pi_{*} b_{t}$, which completes the proof of our theorem.

2.5. The forms $c_{t}, d_{t}$ on $M$

Definition 2.6. Set

$$
\gamma=-\theta^{\mathfrak{p}} \exp \left(\theta^{\mathfrak{p}, 2} / 2 i \pi\right) R(0)
$$

Equivalently, we have

$$
\gamma=-\left\langle R^{\prime}\left(\theta^{\mathfrak{p}, 2} / 2 i \pi\right), \theta^{\mathfrak{p}}\right\rangle
$$

Then $\gamma$ is a real odd form on $M$.

Proposition 2.7. The form $\gamma$ is closed on $M$. 
Proof. This follows from (1.38), (2.51).

Remark 2.8. By replacing $M, S$ by $M \times \mathcal{S}, S \times \mathcal{S}$, by Proposition 2.7, we also deduce that the cohomology class $[\gamma(h)] \in H^{*}(M, \mathbf{R})$ of $\gamma(h)$ does not depend on the choice of $h \in \mathcal{S}$. Moreover, if $h, h^{\prime} \in \mathcal{S}$, there are canonical forms $\widetilde{\gamma}\left(h, h^{\prime}\right) \in \Omega^{\cdot}(M) / d \Omega^{-1}(M)$ such that

$$
d \tilde{\gamma}\left(h, h^{\prime}\right)=\gamma\left(h^{\prime}\right)-\gamma(h)
$$

Let $e_{1}, \ldots, e_{m}$ still denote an orthonormal basis of $T X$. Set

$$
L=\sum_{i=1}^{m} e^{i} \wedge \widehat{e}^{i}
$$

Recall that the map $\varphi$ was defined in Definition 1.10 .

Definition 2.9. For $t \geqslant 0$, set

$$
\begin{aligned}
c_{t} & =-(2 i \pi)^{(m+1) / 2} \varphi \int^{\widehat{B}} \theta^{\mathfrak{p}} \exp \left(-\sigma\left(\mathcal{A}_{t}^{2}\right)\right) R(0), \\
d_{t} & =-(2 i \pi)^{m / 2} \varphi \int_{\widehat{B}}^{\sqrt{t}} \frac{\theta^{\mathfrak{p}} \wedge \widehat{\theta}^{\mathfrak{p}}}{2} \exp \left(-\sigma\left(\mathcal{A}_{t}^{2}\right)\right) R(0), \\
e_{t} & =(2 i \pi)^{m / 2} \varphi \int^{\widehat{B}} \frac{L}{4 \sqrt{t}} \exp \left(-\sigma\left(\mathcal{A}_{t}^{2}\right)\right) R(0) .
\end{aligned}
$$

Then $c_{t}, d_{t}, e_{t}$ are smooth real forms on $M$.

Let $\widehat{\mathcal{X}}$ be the total space of $\widehat{T X}$. Recall that the forms $\underline{a}_{t}, \underline{b}_{t}$ on $\widehat{\mathcal{X}}$ were defined in Definition 1.8. By (1.25), $\sigma_{\widehat{\theta} \mathfrak{p}}$ is the section of $q^{*} \widehat{T^{*} X}$ that is given by

$$
\sigma_{\widehat{\theta} \mathfrak{p}}=2 \pi\left\langle\mu, i \widehat{\theta}^{\mathfrak{p}}\right\rangle \text {. }
$$

We identify $\widehat{T X}$ and $\widehat{T^{*} X}$ by the metric $g^{\widehat{T X}}$, so that $\sigma_{\widehat{\theta}^{\mathfrak{p}}}$ can be viewed as a section of $q^{*} \widehat{T X}$.

Theorem 2.10. The forms $\pi_{*} c_{t}$ are odd, they are closed, and their cohomology class does not depend on $t \geqslant 0$. The forms $\pi_{*} d_{t}, \pi_{*} e_{t}$ are even. Moreover,

$$
\pi_{*} c_{0}=\pi_{*}\left[e\left(T X, \nabla^{T X}\right) \gamma\right] \text {. }
$$

Also,

$$
\frac{\partial}{\partial t} \pi_{*} c_{t}=\frac{1}{t} d \pi_{*} d_{t}, \quad\left(1+2 t \frac{\partial}{\partial t}\right) \psi_{\sqrt{t}} \pi_{*} e_{t}=\psi_{\sqrt{t}} \pi_{*} d_{t} .
$$

The following identities hold:

$$
c_{t}=-q_{*}\left[\left\langle\mu, 2 i \pi \theta^{\mathfrak{p}}\right\rangle\left(\sigma_{\widehat{\theta} \mathfrak{p}}^{*} \underline{a}_{t}\right) \exp (\kappa)\right], \quad d_{t}=-t q_{*}\left[\left\langle\mu, 2 i \pi \theta^{\mathfrak{p}}\right\rangle\left(\sigma_{\widehat{\theta}^{\mathfrak{p}}}^{*} \underline{b}_{t}\right) \exp (\kappa)\right] .
$$

If $\widehat{\theta}^{\mathfrak{p}}$ is nondegenerate, and if $K$ is a compact subset of $M$, there exists $c_{K}>0$ such that, on $K$, as $t \rightarrow+\infty$,

$$
c_{t}=\mathcal{O}\left(e^{-c_{K} t}\right), \quad d_{t}=\mathcal{O}\left(e^{-c_{K} t}\right), \quad e_{t}=\mathcal{O}\left(e^{-c_{K} t}\right) .
$$


Proof. It is easy to see that the forms $\pi_{*} c_{t}$ are odd. Until further notice, we may as well make $2 i \pi=1$, so as to disregard the normalization of the forms involving $\varphi$. By $(2.20)$, (2.21), and (2.31), we get

$$
d c_{t}=-\int^{\widehat{B}} \sqrt{t}\left[\widehat{\theta}^{\mathfrak{p}}, \theta^{\mathfrak{p}}\right] \exp \left(-\sigma\left(\mathcal{A}_{t}^{2}\right)\right) R(0) .
$$

By (2.61), we get

$$
d \pi_{*} c_{t}=-\pi_{*} \int^{\widehat{B}} \sqrt{t}\left[\widehat{\theta}^{\mathfrak{p}}, \theta^{\mathfrak{p}}\right] \exp \left(-\sigma\left(\mathcal{A}_{t}^{2}\right)\right) R(0) .
$$

Proceeding as in (2.42), and using (2.62), we obtain

$$
d \pi_{*} c_{t}=-(-1)^{m} \int_{X} \int^{B} \sqrt{t}\left[\widehat{\theta}^{\mathfrak{p}}, \theta^{\mathfrak{p}}\right] \exp \left(-\sigma\left(\mathcal{A}_{t}^{2}\right)\right) R(0) .
$$

By (2.30), (2.31), we can rewrite (2.63) in the form

$$
d \pi_{*} c_{t}=-(-1)^{m} \widehat{\int}_{X} \int^{B} t \widehat{\mathcal{L}}_{t}\left[\widehat{\theta}^{\mathfrak{p}} \exp \left(-\sigma\left(\mathcal{A}_{t}^{2}\right)\right)\right] R(0) .
$$

Using the Ad-invariance of $R$, we deduce from (2.64) that

$$
d \pi_{*} c_{t}=-(-1)^{m} \widehat{\int}_{X} \widehat{d}^{X} \int^{B} \widehat{t}^{\mathfrak{p}} \exp \left(-\sigma\left(\mathcal{A}_{t}^{2}\right)\right) R(0)=0 .
$$

We have proved that the forms $\pi_{*} c_{t}$ are closed. By universality, their cohomology class does not depend on $t$. By (2.19), (2.51), and (2.55), we get (2.57).

Now, we proceed as in Remark 1.7, while using the corresponding notation. Namely, we enlarge $M, S$ to $M \times \mathbf{R}_{+}^{*}, S \times \mathbf{R}_{+}^{*}$, and, over $M \times\{s\}$, we equip $\widehat{T X}$ with the metric $g^{\widehat{T X}} / s$. In this way, we have a new metric $g^{\widehat{T X}}$ on the vector bundle $\widehat{T X}$. Let $\underline{\mathcal{A}}_{t}$ be the corresponding superconnection on $M \times \mathbf{R}_{+}^{*}$. Set

$$
\underline{c}_{t}=-\int^{\widehat{B}} \theta^{\mathfrak{p}} \exp \left(-\sigma\left(\underline{\mathcal{A}}_{t}^{2}\right)\right) R(0) .
$$

Then $\underline{c}_{t}$ is the analogue of $c_{t}$ on $M \times \mathbf{R}_{+}^{*}$. By the above, the form $\pi_{*} \underline{c}_{t}$ is closed on $S \times \mathbf{R}_{+}^{*}$. Using (1.68), (2.18), we rewrite (2.66) in the form

$$
\underline{c}_{t}=-\int^{\widehat{B}} \theta^{\mathfrak{p}} \exp \left(-\sigma\left(\mathcal{A}_{s t}^{2}\right)-\frac{\sqrt{t} d s}{2 \sqrt{s}} \widehat{\theta}^{\mathfrak{p}}\right) R(0) .
$$

By (2.55), (2.67), we obtain

$$
\underline{c}_{t}=c_{s t}+\frac{d s}{s} d_{s t} .
$$

Since $\pi_{*} \underline{c}_{1}$ is closed, by (2.68), we get the first identity in (2.58). 
By proceeding as in the proof of Theorem 2.5, and using (2.30), (2.55), we get

$$
\begin{aligned}
\left(1+2 t \frac{\partial}{\partial t}\right) \psi_{\sqrt{t}} \pi_{*} e_{t}=(-1)^{m} \psi_{\sqrt{t}} \int_{X} \int^{B} \frac{L}{2} \\
\left(\widehat{\nabla}^{T X \otimes \mathfrak{g}_{r}, u}+2 i_{-\sqrt{t} \mathfrak{f}^{\mathfrak{p}}, X}+\frac{1}{2} T^{0}+\frac{\operatorname{ad}\left(\theta^{\mathfrak{p}}\right)}{\sqrt{t}}\right)\left[\frac{\theta^{\mathfrak{p}}}{2} \exp \left(-\sigma\left(\mathcal{A}_{t}^{2}\right)\right)\right] R(0) .
\end{aligned}
$$

Since $R$ is Ad-invariant, we can ignore the term $\frac{\operatorname{ad}\left(\theta^{\mathfrak{p}}\right)}{\sqrt{t}}$ in the right-hand side of (2.69). Also, by (2.9), (2.22), we get

$$
i_{T^{0}} L=0 .
$$

By (2.69), (2.70), we obtain

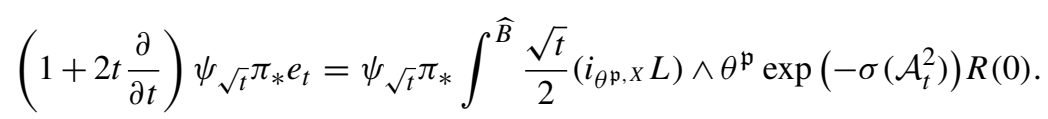

Also,

$$
i_{\theta^{\mathfrak{p}}, X} L=\widehat{\theta}^{\mathfrak{p}}
$$

By (2.55), (2.71), and (2.72), we get the second identity in (2.58).

By proceeding as in the proof of equation (1.89) in Theorem 1.15, we get (2.59).

If $\widehat{\theta}^{\mathfrak{p}}$ is nondegenerate, by proceeding as in the proof of Theorem 1.15, we get $(2.60)$. The proof of our theorem is completed.

\subsection{The $W$-invariant}

In this section, we assume that $\widehat{\theta}^{\mathfrak{p}}$ is nondegenerate. In particular, equation (2.60) in Theorem 2.10 holds. Let $o(T X)$ be the orientation bundle of $T X$. Recall that the form $\psi$ on the total space of $\widehat{T X} \backslash\{0\}$ was defined in Definition 1.9. Then $\sigma_{\widehat{\theta} \mathfrak{p}}^{*} \psi$ is a smooth $(m-1)$-form on $\mathcal{N}$ with values in $o(T X)$. By equation (1.92) in Theorem 1.15,

$$
d\left(\sigma_{\hat{\theta} \mathfrak{p}}^{*} \psi\right)=q^{*} e\left(T X, \nabla^{T X}\right)
$$

By (1.77), by equation (1.89) in Theorem 1.15, and by Theorem 2.5, we obtain

$$
(\pi q)_{*}\left[\left(\sigma_{\hat{\theta} \mathfrak{*}}^{*} \psi\right) \exp (\kappa)\right]=0 .
$$

Definition 2.11. Set

$$
W=-\int_{0}^{+\infty} d_{t} \frac{d t}{t}
$$

Then $W$ is a smooth form on $M$ with values in $o(T X)$.

Theorem 2.12. The following identity holds:

$$
W=q_{*}\left[\left\langle\mu, 2 i \pi \theta^{\mathfrak{p}}\right\rangle\left(\sigma_{\widehat{\theta} \mathfrak{p}}^{*} \psi\right) \exp (\kappa)\right] .
$$

The even form $\pi_{*} W$ on $S$ is such that

$$
d \pi_{*} W=\pi_{*}\left[e\left(T X, \nabla^{T X}\right) \gamma\right] .
$$

If $\operatorname{dim} X$ is odd, the form $\pi_{*} W$ is closed, and its cohomology class $\left[\pi_{*} W\right] \in H^{\cdot}(S, \mathbf{R})$ does not depend on $\left(T^{H} M, g^{T X}\right)$ or on infinitesimal variations of $h$. 
Proof. The first part of our theorem follows from (1.77), from Theorem 2.10, and from (2.75). If $m$ is odd, the right-hand side of (2.77) vanishes, and $\pi_{*} W$ is closed. Replacing $M, S$ by $M \times \mathcal{S}^{\prime}, S \times \mathcal{S}^{\prime}$, it is also obvious that the cohomology class of $\pi_{*} W$ does not depend on the data. The proof of our theorem is completed.

Remark 2.13. If $\operatorname{dim} X$ is even, by replacing $M, S$ by $M \times \mathcal{S}^{\prime}, S \times \mathcal{S}^{\prime}$, we deduce from (2.77) that the infinitesimal variation of $\pi_{*} W$ in $\Omega^{*}(S) / d \Omega^{*}(S)$ with respect to the data in $\mathcal{S}^{\prime}$ can be evaluated in terms of the Chern-Simons secondary classes associated with the Euler class of $T X$, and of the secondary class of forms described in Remark 2.8. Details are left to the reader.

\subsection{The forms $\pi_{*} W$ and adiabatic limits}

Let $\pi^{\prime}: M^{\prime} \rightarrow S$ be another smooth submersion on $S$ with compact fibre $Z$. Let $\tau: M \rightarrow$ $M^{\prime}$ be a smooth submersion with compact fibre $Y$ such that

$$
\pi=\pi^{\prime} \tau \text {. }
$$

Then $\tau$ induces a fibrewise projection $X \rightarrow Z$ with compact fibre $Y$. Let $i: Y \rightarrow X$ be the obvious embedding. Let $g^{T Y}$ be the metric induced by $g^{T X}$ on $T Y$, and let $T^{H} X$ be the bundle orthogonal to $T Y$ in $T X$ with respect to $g^{T X}$. Then $T^{H} M \oplus T^{H} X$ plays the role of $T^{H} M$ for the submersion $\tau: M \rightarrow M^{\prime}$.

To the projection $\tau: M \rightarrow M^{\prime}$ we can associate exactly the same objects as we did for the projection $\pi: M \rightarrow S$. In particular, the form $i^{*} \widehat{\theta}^{\mathfrak{p}}$ is the exact analogue of $\widehat{\theta}^{\mathfrak{p}}$. If $i^{*} \widehat{\theta}^{\mathfrak{p}}$ is nondegenerate, then $\widehat{\theta}^{\mathfrak{p}}$ is nondegenerate.

Let $g^{T Z}$ be a metric on $T Z$. For $\epsilon>0$, let $g_{\epsilon}^{T X}$ be the metric on $T X=T Y \oplus T^{H} X$,

$$
g_{\epsilon}^{T X}=g^{T Y} \oplus \frac{1}{\epsilon} \tau^{*} g^{T Z} .
$$

Assume that $i^{*} \widehat{\theta}^{\mathfrak{p}}$ is nondegenerate. To the projection $\pi: M \rightarrow S$, and to $\left(T^{H} M, g_{\epsilon}^{T X}\right)$, we can associate the even form $W_{\epsilon}$ on $M$ as in Definition 2.11. Similarly, to the projection $\tau: M \rightarrow M^{\prime}$, and to $\left(T^{H} M \oplus T^{H} X, g^{T Y}\right)$, we can associate the even form $\underline{W}$ on $M$.

If $X$ is odd dimensional and $Z$ is even dimensional, $\tau_{*} \underline{W}$ is a closed form on $M^{\prime}$, and so $\pi_{*}^{\prime}\left[e(T Z) \tau_{*} \underline{W}\right] \in H^{\text {even }}(S, \mathbf{R})$ is well defined. If $Z$ is odd dimensional, $e(T Z)=0$. If $X$ is odd dimensional and $Z$ is odd dimensional, we adopt the natural convention

$$
e(T Z) \tau_{*} \underline{W}=0 .
$$

As before, convergence of forms means here uniform convergence of the forms and their derivatives of arbitrary order over compact subsets.

Theorem 2.14. As $\epsilon \rightarrow 0$, then

$$
W_{\epsilon} \rightarrow W_{0}=\tau^{*}\left[e\left(T Z, \nabla^{T Z}\right)\right] \underline{W} .
$$

If $X$ is odd dimensional, then

$$
\left[\pi_{*} W\right]=\pi_{*}^{\prime}\left[e(T Z) \tau_{*} \underline{W}\right] \quad \text { in } H^{\text {even }}(S, \mathbf{R}) .
$$


Proof. We add the extra subscript $\epsilon$ to the objects considered in the previous subsections to mark their dependence on the metric $g_{\epsilon}^{T X}$. By (2.75), we get

$$
W_{\epsilon}=-\int_{0}^{+\infty} d_{t, \epsilon} \frac{d t}{t} .
$$

Let $\underline{d}_{t}$ be the form on $M$ that is attached to $\tau$ and to $\left(T^{H} M \oplus T^{H} X, g^{T Y}\right)$. We claim that, as $\epsilon \rightarrow 0$, we have the uniform convergence over compact subsets of $M \times \mathbf{R}_{+}$of smooth forms and their derivatives on $M$ of arbitrary order

$$
\frac{d_{t, \epsilon}}{t} \rightarrow \tau^{*}\left[e\left(T Z, \nabla^{T Z}\right)\right] \frac{d_{t}}{t}
$$

and also that, if $K \subset M$ is compact, there exist $c_{K}>0, C_{K}>0$ such that, on $K$, for $t \geqslant 0,0<\epsilon \leqslant 1$,

$$
\left|d_{t, \epsilon}\right| \leqslant C_{K} \exp \left(-c_{K} t\right)
$$

Then (2.81) follows from (2.84), (2.85). So we concentrate on the proof of these two equations.

We consider the analogue of equation (2.55) for $d_{t, \epsilon}$. Let $e_{1}, \ldots, e_{m^{\prime}}$ be an orthonormal basis of $T Y$, and let $e_{m^{\prime}+1}, \ldots, e_{m}$ be an orthonormal basis of $T Z$. Then $e_{1}, \ldots, e_{m^{\prime}}, \sqrt{\epsilon} e_{m^{\prime}+1}^{H}, \ldots, \sqrt{\epsilon} e_{m}^{H}$ is an orthonormal basis of $T X$ with respect to the metric $g_{\epsilon}^{T X}$. Let $\int^{\widehat{B}, Y}, \int^{\widehat{B}, Z}$ denote the Berezin integrals on $\Lambda^{\cdot}\left(\widehat{T^{*} Y}\right), \Lambda^{\cdot}\left(\widehat{T^{*} Z}\right)$ with respect to the metrics $g^{\widehat{T Y}}, g^{\widehat{T Z}}$. Let $\int_{\epsilon}^{\widehat{B}}$ denote the Berezin integral on $\Lambda \cdot(\widehat{T * X})$ with respect to the metric $g_{\epsilon}^{\widehat{T X}}$. One has the obvious formula

$$
\int_{\epsilon}^{\widehat{B}}=\epsilon^{\left(m-m^{\prime}\right) / 2} \int^{\widehat{B}, Y} \int^{\widehat{B}, Z} .
$$

Let $\nabla_{\epsilon}^{T X}$ be the Euclidean connection on $T X$ attached to $\left(T^{H} M, g_{\epsilon}^{T X}\right)$, let $\nabla^{T Y}$ be the Euclidean connection on $T Y$ attached to $\left(T^{H} M \oplus T^{H} X, g^{T Y}\right)$, and let $\nabla^{T Z}$ be the Euclidean connection on $T Z$ attached to $T^{H} M^{\prime}, g^{T Z}$. By proceeding as in [6, pp. 116-117], $[12, \S 1.2]$, as $\epsilon \rightarrow 0$, we have the convergence of connections on $T X=T Y \oplus T^{H} X \simeq$ $T Y \oplus \tau^{*} T Z$,

$$
\nabla_{\epsilon}^{T X} \rightarrow \nabla_{0}^{T X}=\left[\begin{array}{cc}
\nabla^{T Y} & * \\
0 & \tau^{*} \nabla^{T Z}
\end{array}\right]
$$

In (2.87), the precise value of $*$ is irrelevant.

Moreover, as $\epsilon \rightarrow 0$, one has the easy

$$
\left|\widehat{\theta}^{\mathfrak{p}}\right|_{\epsilon}^{2} \rightarrow\left|i^{*} \widehat{\theta}^{\mathfrak{p}}\right|^{2}
$$

and also

$$
\left|\widehat{\theta}^{\mathfrak{p}}\right|_{\epsilon}^{2} \geqslant\left|i^{*} \widehat{\theta}^{\mathfrak{p}}\right|^{2}>0
$$

Using (2.19), (2.55), and the above considerations, it is now easy to get (2.84) and (2.85). We have completed the proof of (2.81).

If $X$ is odd dimensional, by Theorem 2.12, the cohomology class of $\pi_{*} W$ does not depend on $\left(T^{H} M, g^{T X}\right)$. By $(2.81)$, as $\epsilon \rightarrow 0$, we get

$$
\pi_{*} W_{\varepsilon} \rightarrow \pi_{*}^{\prime}\left[e\left(T Z, \nabla^{T Z}\right) \tau_{*} \underline{W}\right]
$$

from which we get (2.82). The proof of our theorem is completed. 
Remark 2.15. If $X$ is even dimensional, by Theorem 2.12 and Remark 2.13, in $\Omega^{\cdot}(S) / d \Omega^{-1}(S)$, the dependence of $\pi_{*} W$ on the metrics can be explicitly calculated in terms of secondary classes. By proceeding as in the proof of Theorem 2.14, we can give an explicit formula for $\pi_{*} W-\pi_{*}^{\prime}\left[e\left(T Z, \nabla^{T Z}\right) \tau_{*} \underline{W}\right]$ in $\Omega^{\cdot}(S) / d \Omega^{-1}(S)$ in terms of such secondary classes. Details are left to the reader.

Let $T^{H} M^{\prime}$ be a horizontal subbundle of $T M^{\prime}$, and let $g^{T Z}$ be a metric on $T Z$. Let $p^{\prime}: P_{G}^{\prime} \rightarrow M^{\prime}$ be a principal $G$-bundle having the same properties as $p: P_{G} \rightarrow M$. We denote with a prime the objects constructed before that are associated with the projection $\pi^{\prime}$. We assume that

$$
P_{G}=\tau^{*} P_{G}^{\prime}
$$

Then

$$
\widehat{\theta}^{\mathfrak{p}}=\tau^{*} \widehat{\theta}^{\mathfrak{p}}
$$

If $\widehat{\theta}^{\mathfrak{p} \prime}$ is nondegenerate, then $\widehat{\theta}^{\mathfrak{p}}$ is nondegenerate.

Assume that $\widehat{\theta}^{p^{\prime}}$ is nondegenerate. Let $W^{\prime}$ be the form on $M^{\prime}$ that is associated with $\left(T^{H} M^{\prime}, g^{T Z}\right)$. For $\epsilon>0$, we equip $T X=T Y \oplus T^{H} X$ with the metric $g^{T X, \epsilon}$ that is given by

$$
g^{T X, \epsilon}=\epsilon g^{T Y} \oplus \tau^{*} g^{T Z} .
$$

Let $W^{\epsilon}$ be the form on $M$ that is associated with $\left(T^{H} M, g^{T X, \epsilon}\right)$.

Theorem 2.16. As $\epsilon \rightarrow 0$, then

$$
W^{\epsilon} \rightarrow W^{0}=e\left(T Y, \nabla^{T Y}\right) \tau^{*} W^{\prime}
$$

If $X$ is odd dimensional, then

$$
\left[\pi_{*} W\right]=\chi(Y)\left[\pi_{*}^{\prime} W^{\prime}\right] \quad \text { in } H^{\text {even }}(S, \mathbf{R}) .
$$

Proof. We add the extra superscript $\epsilon$ to the objects considered in the previous subsections to mark their dependence on the metric $g^{T X, \epsilon}$. By (2.75), we get

$$
W^{\epsilon}=-\int_{0}^{+\infty} d_{t}^{\epsilon} \frac{d t}{t}
$$

We claim that, as $\epsilon \rightarrow 0$, we have the convergence of forms

$$
\frac{d_{t}^{\epsilon}}{t} \rightarrow e\left(T Y, \nabla^{T Y}\right) \tau^{*} \frac{d_{t}^{\prime}}{t},
$$

and also that the analogue of (2.85) holds; i.e., for $t \geqslant 0,0<\epsilon \leqslant 1$,

$$
\left|d_{t}^{\epsilon}\right| \leqslant C_{K} \exp \left(-c_{K} t\right)
$$

Then (2.94) follows from $(2.97)$, (2.98). Now, we will prove these two equations.

Note that

$$
g^{T X, \epsilon}=\epsilon g_{\epsilon}^{T X} .
$$


We use the same notation as in the proof of Theorem 2.14. Let $\int^{\widehat{B}, \epsilon}$ be the Berezin integral on $\left.\Lambda \cdot \widehat{T^{*} X}\right)$ with respect to the metric $g^{T X, \epsilon}$. Instead of $(2.86)$, we get

$$
\int^{\widehat{B}, \epsilon}=\epsilon^{-m^{\prime} / 2} \int^{\widehat{B}, Y} \int^{\widehat{B}, Z} .
$$

By (2.99), $\nabla_{\epsilon}^{T X}$ is the connection on $T X$ that is associated with $\left(g^{T X, \epsilon}, T^{H} M\right)$, and (2.87) still holds. Also, by (2.92),

$$
\left|\widehat{\theta}^{\mathfrak{p}}\right|^{\epsilon, 2}=\tau^{*}\left|\widehat{\theta}^{\mathfrak{p} \prime}\right|^{2}
$$

Using (2.19), (2.55), and the above considerations, we get (2.97), (2.98).

If $X$ is odd dimensional, proceeding as in the proof of Theorem 2.14, and using (2.94), we get

$$
\left[\pi_{*} W\right]=\pi_{*}^{\prime}\left[\tau_{*}\left[e\left(T Y, \nabla^{T Y}\right)\right] W^{\prime}\right] \quad \text { in } H^{\text {even }}(S, \mathbf{R}) .
$$

By (2.102), we get (2.95). The proof of our theorem is completed.

\subsection{Rigidity}

Up to now, the flat structure on the principal $G$-bundle $P_{G}$ was fixed. Let $\mathcal{F}$ be the Fréchet manifold of such flat structures. We will replace $M, S$ by $M \times \mathcal{S}^{\prime} \times \mathcal{F}, S \times \mathcal{S}^{\prime} \times \mathcal{F}$. The restriction of $P_{G}$ to the fibres over $\mathcal{F}$ of $M \times \mathcal{S}^{\prime} \times \mathcal{F}$ is equipped with a flat connection. However, the principal $G$-bundle $P_{G}$ is no longer flat on $M \times \mathcal{S}^{\prime} \times \mathcal{F}$.

Proposition 2.17. In degree $\geqslant 3$, the cohomology class of $\gamma$ is invariant under deformation of the flat structure on $P_{G}$.

Proof. We may as well make $2 i \pi=1$. We proceed as in [15, Proof of Theorem 2.1]. Let $d^{\mathcal{F}}$ be the de Rham operator on $\mathcal{F}$. By $(2.51)$, we get

$$
d^{\mathcal{F}} \gamma=\left(-d^{\mathcal{F}} \theta^{\mathfrak{p}}+\theta^{\mathfrak{p}}\left(d^{\mathcal{F}} \theta^{\mathfrak{p}, 2}\right)\right) \exp \left(\theta^{\mathfrak{p}, 2}\right) R(0)
$$

By (1.39), we have the identity

$$
d^{\mathcal{F}} \theta^{\mathfrak{p}, 2}=\left[d+\theta^{\mathfrak{k}}, d^{\mathcal{F}} \theta^{\mathfrak{k}}\right] .
$$

By (1.38), (2.104), and using the Ad-invariance of $R$, we get

$$
\theta^{\mathfrak{p}}\left(d^{\mathcal{F}} \theta^{\mathfrak{p}, 2}\right) \exp \left(\theta^{\mathfrak{p}, 2}\right) R(0)=-d\left[\theta^{\mathfrak{p}} d^{\mathcal{F}} \theta^{\mathfrak{k}} \exp \left(\theta^{\mathfrak{p}, 2}\right)\right] R(0) .
$$

Set

$$
k(x)=\frac{\exp \left(x^{2}\right)-1}{x^{2}}
$$

Then

$$
\left[\left(d^{\mathcal{F}} \theta^{\mathfrak{p}}\right) \exp \left(\theta^{\mathfrak{p}, 2}\right) R(0)\right]^{(\geqslant 3)}=\left(d^{\mathcal{F}} \theta^{\mathfrak{p}}\right) \theta^{\mathfrak{p}, 2} k\left(\theta^{\mathfrak{p}, 2}\right) R(0) .
$$

Also, since $R$ is Ad-invariant, we get

$$
\left(d^{\mathcal{F}} \theta^{\mathfrak{p}}\right) \theta^{\mathfrak{p}, 2} k\left(\theta^{\mathfrak{p}, 2}\right) R(0)=-\frac{1}{2}\left[\theta^{\mathfrak{p}}, d^{\mathcal{F}} \theta^{\mathfrak{p}}\right] \theta^{\mathfrak{p}} k\left(\theta^{\mathfrak{p}, 2}\right) R(0) .
$$


By (1.38), (2.104), and (2.108), we obtain

$$
\begin{aligned}
\left(d^{\mathcal{F}} \theta^{\mathfrak{p}}\right) \theta^{\mathfrak{p}, 2} k\left(\theta^{\mathfrak{p}, 2}\right) R(0) & =\frac{1}{2}\left(d^{\mathcal{F}} \theta^{\mathfrak{p}, 2}\right) \theta^{\mathfrak{p}} k\left(\theta^{\mathfrak{p}, 2}\right) R(0) \\
& =\frac{1}{2} d\left[\left(d^{\mathcal{F}} \theta^{\mathfrak{k}}\right) \theta^{\mathfrak{p}} k\left(\theta^{\mathfrak{p}, 2}\right)\right] R(0) .
\end{aligned}
$$

Our proposition follows from (2.103), (2.105), (2.107), and (2.109).

Remark 2.18. The proof of Proposition 2.17 shows that, if $\ell \in[0,1] \rightarrow f_{\ell} \in \mathcal{F}$ is a smooth path, there is an even form $\tilde{\gamma}$ on $M$ of degree $\geqslant 2$ such that

$$
d \widetilde{\gamma}=\gamma\left(f_{1}\right)^{(\geqslant 3)}-\gamma\left(f_{0}\right)^{(\geqslant 3)} .
$$

By proceeding as in [15, Proof of Theorem 2.5], one can show that, in degree $\geqslant 4$, the class of $\tilde{\gamma} \in \Omega^{\cdot}(M) / d \Omega^{-1}(M)$ does not depend on the path.

By Theorem 2.10, and by Proposition 2.17, in degree $\geqslant 3$, the common cohomology class of the $\pi_{*} c_{t}, t \geqslant 0$ is invariant under deformation of the flat structure on $P_{G}$. As we shall see in Theorem 7.6, it is a consequence of the main result of this paper that, if $\operatorname{dim} X$ is odd, in degree $\geqslant 2$, the cohomology class of $\pi_{*} W$ is also invariant under deformation of the flat structure on $P_{G}$.

\section{A class of Toeplitz operators}

Let $N$ be a compact Kähler manifold taken as in $\S 1.4$. Assume that there is a $U$-equivariant holomorphic Hermitian line bundle $L$ such that $c_{1}\left(L, g^{L}\right)=\eta$. The purpose of this section is to express the action of elements of length $\leqslant 2$ in $U \mathfrak{g}_{\mathbf{C}}$ on $H^{(0,0)}\left(N, L^{p}\right)$ as Toeplitz operators. We obtain in this way the asymptotics of this action as $p \rightarrow+\infty$. We also compute the asymptotics of the trace of $g=e^{A / p}, A \in \mathfrak{g}_{\mathbf{C}}$ on $H^{(0,0)}\left(N, L^{p}\right)$ in terms of the function $R(A)$ that was introduced in $\S 1.4$.

This section is organized as follows. In $\S 3.1$, we introduce the line bundle $L$, and we express certain Lie derivative operators coming from $\mathfrak{u}$ and acting on $H^{(0,0)}(N, L)$, and the composition of two of these, as Toeplitz operators.

In $\S 3.3$, if $\beta \in \widehat{E}^{*} \otimes \mathfrak{p}$ is nondegenerate, we give a lower bound for the action of $|\beta|^{2}$ on $H^{(0,0)}\left(N, L^{p}\right)$ as $p \rightarrow+\infty$.

In $\S 3.4$, we obtain the asymptotics of the trace of $e^{A / p}$ on $H^{(0,0)}\left(N, L^{p}\right)$ using the Kirillov formula.

Finally, in $\S 3.5$, we compute the asymptotics as $p \rightarrow+\infty$ of the action of certain heat operators.

\subsection{A line bundle on $N$}

We use the assumptions and notation of $\S \S 1.1$ and 1.4. In particular, $N$ denotes a compact complex manifold, $\eta$ is a smooth real closed $U$-invariant $(1,1)$-form on $N$, and $\mu: N \rightarrow \mathfrak{u}^{*}$ is the moment map associated with the action of the compact Lie group $U$ on $N$. Let $g^{T N}$ be a $U$-invariant Hermitian metric on the holomorphic tangent bundle $T N$, and let $d v_{N}$ be the associated volume form on $N$.

In what follows, we assume that $\eta$ is positive; i.e., if $A \in T N$, then $-i \eta(A, \bar{A})$ defines a Hermitian metric on $T N$. 
Let $L$ be a holomorphic line bundle on $N$, let $g^{L}$ be a Hermitian metric on $L$, and let $\nabla^{L}$ denote the corresponding holomorphic Hermitian connection. Let $r^{L}$ be the curvature of $\nabla^{L}$. Let $c_{1}\left(L, g^{L}\right)$ be the corresponding first Chern form; i.e.,

$$
c_{1}\left(L, g^{L}\right)=-\frac{r^{L}}{2 i \pi} .
$$

We assume that

$$
c_{1}\left(L, g^{L}\right)=\eta
$$

Also, we assume that the holomorphic action of $U$ on $N$ lifts to a holomorphic unitary action on $L$.

We equip $C^{\infty}(N, L)$ with the Hermitian product associated with the metrics $g^{T N}, g^{L}$, so that, if $s, s^{\prime} \in C^{\infty}(N, L)$,

$$
\left\langle s, s^{\prime}\right\rangle=(2 \pi)^{-n} \int_{N}\left\langle s, s^{\prime}\right\rangle_{L} d v_{N} .
$$

Then the action of $U$ on $C^{\infty}(N, L)$ is unitary. We equip $H^{(0,0)}(N, L)$ with the Hermitian metric induced by $(3.3)$, so that $U$ acts unitarily on $H^{(0,0)}(N, L)$.

If $A \in \mathfrak{u}$, let $\mathrm{L}_{A}^{L}$ denote the natural action of $A$ on the smooth sections of $L$. Then $\mathrm{L}_{A}^{L}$ is a lift of the holomorphic vector field $A^{N}$ on $N$. Finally, we assume that, if $A \in \mathfrak{u}$,

$$
\mathrm{L}_{A}^{L}=\nabla_{A^{N}}^{L}-2 i \pi\langle\mu, A\rangle .
$$

Equation (1.20) on $\mu$ follows from (3.4). Set

$$
\mathrm{L}_{A^{(1,0)}}^{L}=\nabla_{A^{N(1,0)}}^{L}-2 i \pi\langle\mu, A\rangle, \quad \mathrm{L}_{A^{(0,1)}}^{L}=\nabla_{A^{N(0,1)}}^{L} .
$$

Then

$$
\mathrm{L}_{A}^{L}=\mathrm{L}_{A^{(1,0)}}^{L}+\mathrm{L}_{A^{(0,1)}}^{L} .
$$

Also, if $A, B \in \mathfrak{u}$,

$$
\begin{array}{lr}
{\left[\mathrm{L}_{A}^{L}, \mathrm{~L}_{B}^{L}\right]=-\mathrm{L}_{[A, B]}^{L},} & {\left[\mathrm{~L}_{A^{(1,0)}}^{L}, \mathrm{~L}_{B^{(0,1)}}^{L}\right]=0,} \\
{\left[\mathrm{~L}_{A^{(1,0)}}^{L}, \mathrm{~L}_{B^{(1,0)}}^{L}\right]=-\mathrm{L}_{[A, B]^{(1,0)}}^{L},} & {\left[\mathrm{~L}_{A^{(0,1)}}^{L}, \mathrm{~L}_{B^{(0,1)}}^{L}\right]=-\mathrm{L}_{[A, B]^{(0,1)}}^{L} .}
\end{array}
$$

By (1.21), (3.2), if $A, B \in \mathfrak{u}$,

$$
\langle\mu,[A, B]\rangle=c_{1}\left(L, g^{L}\right)\left(A^{N}, B^{N}\right) .
$$

As explained in $\S 1.4$, the action of $U$ on $N$ extends to a holomorphic action of $G_{\mathbf{C}}$ on $N$. In the same way, the action of $U$ on $L$ extends to a holomorphic action of $G_{\mathbf{C}}$ on $L$. If $A \in \mathfrak{g}_{\mathbf{C}}=\mathfrak{u} \otimes_{\mathbf{R}} \mathbf{C}$, the corresponding Lie derivative operator $L_{A}$ is given by (3.4), and the identities in (3.5), (3.7) still hold.

If $A \in \mathfrak{u}$, the action of $\mathrm{L}_{A}^{L}$ on $C^{\infty}(N, L)$ is formally skew-adjoint, and the action of $\mathrm{L}_{i A}^{L}$ is formally self-adjoint. By (3.4), we see that, if $A \in \mathfrak{u}$,

$$
\left(g^{L}\right)^{-1} \mathrm{~L}_{A}^{L} g^{L}=0, \quad\left(g^{L}\right)^{-1} \mathrm{~L}_{i A}^{L} g^{L}=-4 \pi\langle\mu, A\rangle .
$$




\subsection{Lie derivatives and Toeplitz operators}

The action of $G_{\mathbf{C}}$ on $C^{\infty}(N, L)$ induces a corresponding action on $H^{(0,0)}(N, L)$. As we saw before, the action of $U$ on $H^{(0,0)}(N, L)$ is unitary. If $A \in \mathfrak{g}_{\mathbf{C}}$, the operator $\mathrm{L}_{A}^{L}$ acts on $H^{(0,0)}(N, L)$. By $(3.5)$,

$$
\left.\mathrm{L}_{A}^{L}\right|_{H^{(0,0)}(N, L)}=\left.L_{A^{(1,0)}}^{L}\right|_{H^{(0,0)}(N, L)} .
$$

Let $P$ be the orthogonal projection operator from $C^{\infty}(N, L)$ on $H^{(0,0)}(N, L)$.

Let $\nabla^{T N}$ be the holomorphic Hermitian connection on $T N$, and let $R^{T N}$ be its curvature. If $A \in \mathfrak{u}, \nabla^{T N^{\prime}} A^{N(1,0)}$ is a skew-adjoint endomorphism of $T N$.

Set

$$
d=\operatorname{det} T N .
$$

The metric $g^{T N}$ induces a Hermitian metric $g^{d}$ on $d$. Let $\nabla^{d}$ denote the corresponding holomorphic Hermitian connection on $d$. Then

$$
c_{1}\left(d, g^{d}\right)=-\frac{1}{2 i \pi} \operatorname{Tr}\left[R^{T N}\right] .
$$

The group $U$ acts holomorphically and isometrically on $d$, and this action extends to a holomorphic action of $G_{\mathbf{C}}$. Let $v: N \rightarrow \mathfrak{u}^{*}$ be the map such that, if $A \in \mathfrak{u}$,

$$
\langle v, A\rangle=\frac{1}{2 i \pi} \operatorname{Tr}\left[\nabla^{T N^{\prime}} A^{N(1,0)}\right] .
$$

We use the same notation as in (3.4)-(3.7) for $d$ as for $L$. Then $v$ is a moment map associated with the action of $U$ on $d$. As in (3.4), if $A \in \mathfrak{g}_{\mathbf{C}}$, we get

$$
\mathrm{L}_{A}^{d}=\nabla_{A^{N}}^{d}-2 i \pi\langle v, A\rangle .
$$

Also, the analogues of (3.5)-(3.7) hold. Instead of (1.22), if $A \in \mathfrak{g}_{\mathbf{C}}$, we have

$$
\bar{\partial}\langle v, A\rangle-i_{A^{N(1,0)}} c_{1}\left(d, g^{d}\right)=0 .
$$

Also, the analogue of (3.8) says that, if $A, B \in \mathfrak{u}$,

$$
\langle\nu,[A, B]\rangle=c_{1}\left(d, g^{d}\right)\left(A^{N}, B^{N}\right) .
$$

Theorem 3.1. If $A \in \mathfrak{u}$, the following identity holds:

$$
\left.\mathrm{L}_{A}^{L}\right|_{H^{(0,0)}(N, L)}=-2 i \pi P\langle\mu+v, A\rangle P .
$$

If $A, B \in \mathfrak{u}$, then

$$
\begin{aligned}
\left.\mathrm{L}_{A}^{L} \mathrm{~L}_{B}^{L}\right|_{H^{(0,0)}(N, L)}= & P\left(-4 \pi^{2}\langle\mu+v, A\rangle\langle\mu+v, B\rangle\right. \\
& \left.+2 i \pi c_{1}\left(L \otimes d, g^{L \otimes d}\right)\left(A^{N(0,1)}, B^{N(1,0)}\right)\right) P .
\end{aligned}
$$

Proof. To establish (3.17), we use the first equation in (3.5). The formal adjoint $\nabla_{A^{N(1,0)}}^{L *}$ of $\nabla_{A^{N(1,0)}}^{L}$ acting on $C^{\infty}(N, L)$ is given by

$$
\nabla_{A^{N(1,0)}}^{L *}=-\nabla_{A^{N(0,1)}}^{L}-\operatorname{Tr}\left[\nabla^{T N \prime \prime} A^{N(0,1)}\right] .
$$


Also, $\nabla_{A^{N(0,1)}}^{L}$ vanishes on $H^{(0,0)}(N, L)$, so that, if $s, s^{\prime} \in H^{(0,0)}(N, L)$, then

$$
\left\langle\nabla_{A^{N(1,0)}}^{L} s, s^{\prime}\right\rangle=\left\langle-2 i \pi\langle v, A\rangle s, s^{\prime}\right\rangle
$$

Equation (3.17) follows from the above considerations.

If $s, s^{\prime} \in H^{(0,0)}(N, L)$, by (3.17), we get

$$
\left\langle\mathrm{L}_{A}^{L} \mathrm{~L}_{B}^{L} s, s^{\prime}\right\rangle=-2 i \pi\left\langle\langle\mu+v, A\rangle \mathrm{L}_{B}^{L} s, s^{\prime}\right\rangle .
$$

By (3.5), we obtain

$$
\begin{aligned}
-2 i \pi\langle\mu+v, A\rangle \mathrm{L}_{B}^{L} s= & -4 \pi^{2}\langle\mu+v, A\rangle\langle\mu, B\rangle s \\
& +\left(\nabla_{B^{N(1,0)}} 2 i \pi\langle\mu+v, A\rangle\right) s-\nabla_{B^{N(1,0)}}^{L}(2 i \pi\langle\mu+v, A\rangle s) .
\end{aligned}
$$

By (1.22), (3.15), we get

$$
\nabla_{B^{N(1,0)}}\langle\mu+v, A\rangle=c_{1}\left(L \otimes d, g^{L \otimes d}\right)\left(A^{N(0,1)}, B^{N(1,0)}\right) .
$$

Using (3.19), we also have

$$
\left\langle-\nabla_{B^{N(1,0)}}^{L}(2 i \pi\langle\mu+v, A\rangle s), s^{\prime}\right\rangle=\left\langle-4 \pi^{2}\langle\mu+v, A\rangle\langle v, B\rangle s, s^{\prime}\right\rangle .
$$

By (3.21)-(3.24), we get (3.18). The proof of our theorem is completed.

Remark 3.2. By (3.8), (3.16), equation (3.18) is compatible with (3.7). Moreover, the operators in (3.17), (3.18) are Toeplitz operators. Equation (3.18) expresses the product of two Toeplitz operators of the type (3.17) as a Toeplitz operator. By (3.17), the operators $\left.L_{A}^{L}\right|_{H^{(0,0)}(N, L)}$ form a finite-dimensional Lie algebra of Toeplitz operators. Questions connected with Toeplitz operators will be dealt with in much more detail in $\S 9$.

For $p \in \mathbf{N}$, in Theorem 3.1, we can replace $L$ by $L^{p}$, so that $\mu$ is replaced by $p \mu$. Let $P_{p}$ be the corresponding orthogonal projection operator from $C^{\infty}\left(N, L^{p}\right)$ on $H^{(0,0)}\left(N, L^{p}\right)$.

By Kodaira's vanishing theorem, for $p \in \mathbf{N}$ such that $c_{1}\left(L^{p} \otimes d, g^{L^{p} \otimes d}\right)>0$, for $i>0$, $H^{(0, i)}\left(N, L^{p}\right)$ vanishes.

\subsection{The action of $\underline{\beta}^{2}$ on $H^{(0,0)}(N, L)$}

We make the same assumptions as in $\S \S 1.3$ and 1.4 , and we use the corresponding notation. We still take $\beta \in \widehat{E}^{*} \otimes \mathfrak{p}$. Recall that $|\underline{\beta}|^{2}$ was defined in (1.12), that $|\underline{\beta}|^{2} \in$ $U \mathfrak{g} \cap U \mathfrak{u}$, and that (1.14) holds.

For $p \in \mathbf{N}$, let $\rho_{p}$ be the representation of $G_{\mathbf{C}}$ on $H^{(0,0)}\left(N, L^{p}\right)$. For $p \in \mathbf{N}, \rho_{p}|\underline{\beta}|^{2}$ is self-adjoint and nonnegative in $\operatorname{End}\left(H^{(0,0)}\left(N, L^{p}\right)\right)$, and, moreover,

$$
\rho_{p}|\underline{\beta}|^{2}=\left.\sum_{i=1}^{m} \mathrm{~L}_{\beta\left(e_{i}\right)}^{L^{p}, 2}\right|_{H^{(0,0)}\left(N, L^{p}\right)} .
$$

Theorem 3.3. There exist $C^{\prime}>0, C^{\prime \prime}>0$ such that, for $p \in \mathbf{N}$,

$$
\rho_{p}|\underline{\beta}|^{2} \geqslant p^{2} P_{p}\left|\sigma_{\beta}\right|_{\widehat{E}^{*}}^{2} P_{p}-C^{\prime} p-C^{\prime \prime}
$$

If $\beta$ is nondegenerate, there exist $c>0, C>0$ such that, for $p \in \mathbf{N}$,

$$
\rho_{p}|\underline{\beta}|^{2} \geqslant c p^{2}-C \text {. }
$$


Proof. By equation (3.18) in Theorem 3.1, and by (3.25), we get

$$
\begin{aligned}
\rho_{p}|\underline{\beta}|^{2}= & 4 \pi^{2} P_{p} \sum_{j=1}^{m}\left|\left\langle p \mu+v, i \beta\left(e_{j}\right)\right\rangle\right|^{2} P_{p} \\
& -P_{p} \sum_{j=1}^{m} 2 i \pi c_{1}\left(L^{p} \otimes d, g^{L^{p} \otimes d}\right)\left(\beta\left(e_{j}\right)^{N(0,1)}, \beta\left(e_{j}\right)^{N(1,0)}\right) P_{p} .
\end{aligned}
$$

From (3.28), we get (3.26). If $\beta$ is nondegenerate, (3.27) follows from (3.26). The proof of our theorem is completed.

Remark 3.4. If $T$ is a maximal torus in $U$, if $N=U / T$, and if $L$ is the holomorphic line bundle on $N$ associated with the weight $\lambda$, from (3.28), one recovers the formula for the action of the Casimir operator of $U$ on $H^{(0,0)}\left(N, L^{p}\right)$.

\subsection{The asymptotics of the trace of $e^{A / p}$}

If $B$ is a complex $(m, m)$ matrix, put

$$
|B|=\sup _{\lambda \in \operatorname{Sp}(B)}|\lambda| .
$$

If $B$ is such that $|B|<2 \pi$, set

$$
\operatorname{Td}(B)=\operatorname{det}\left[\frac{B}{1-e^{-B}}\right] .
$$

If $B$ is self-adjoint, no condition on $B$ is necessary to define $\operatorname{Td}(B)$.

From the above, there exists $\epsilon>0$ such that, if $A \in \mathfrak{g}_{\mathbf{C}},|A|<\epsilon$, the form

$$
\operatorname{Td}_{A}\left(N, g^{T N}\right)=\mathrm{Td}\left[-\frac{R^{T N}}{2 i \pi}+\nabla^{T N^{\prime}} A^{N(1,0)}\right]
$$

is well defined. Note that, if $A \in i \mathfrak{u}$, no condition on $A$ is needed.

For $g \in G_{\mathbf{C}}$, set

$$
\chi_{p}(g)=\operatorname{Tr}^{H^{(0,0)}\left(N, L^{p}\right)}[g] .
$$

We now give a form of Kirillov's formula.

Theorem 3.5. For $p \in \mathbf{N}$ large enough, for $A \in \mathfrak{g}_{\mathbf{C}},|A| \leqslant \epsilon$,

$$
\chi_{p}\left[e^{A}\right]=\int_{N} \operatorname{Td}_{A}\left(T N, g^{T N}\right) \exp \left(2 i \pi p\langle\mu, A\rangle+p c_{1}\left(L, g^{L}\right)\right) .
$$

Proof. For $A \in \mathfrak{u}$, this is just the Kirillov formula [4, Theorem 8.2]. Equation (3.33) extends by analytic continuation to the general case.

Recall that, for $A \in \mathfrak{g}_{\mathbf{C}}, R(A)$ was defined in (1.23). Using (3.2), we get

$$
R(A)=\int_{N} \exp \left(2 i \pi\langle\mu, A\rangle+c_{1}\left(L, g^{L}\right)\right) .
$$

As in $\S 1.8$, we identify a neighbourhood of 0 in $\mathfrak{g}_{\mathbf{C}}$ with a neighbourhood of 1 in $G_{\mathbf{C}}$. For $a \in \mathbf{R}$, we define the action of $\delta_{a}$ on $C^{\infty}\left(\mathfrak{g}_{\mathbf{C}}, \mathbf{C}\right)$ as in (1.57). 
Theorem 3.6. As $p \rightarrow+\infty$,

$$
\left(\delta_{1 / p} \chi_{p}\right)(A)=p^{n} R(A)+\mathcal{O}\left(p^{n-1}\right) .
$$

Proof. This is a trivial consequence of equation (3.33) in Theorem 3.5.

\subsection{A heat operator acting on $\chi_{p}$}

Let $\mathscr{H}\left(G_{\mathbf{C}}\right)$ be the vector space of complex-valued holomorphic functions on $G_{\mathbf{C}}$. As in $\S 1.1$, we identify $U \mathfrak{g}_{\mathbf{C}}$ with the algebra of left-invariant complex holomorphic differential operators on $G_{\mathbf{C}}$, which acts on $\mathscr{H}\left(G_{\mathbf{C}}\right)$. Similarly, $U \mathfrak{u}$ can be identified with the algebra of left-invariant real differential operators on $U$, which acts on $C^{\infty}(U, \mathbf{R})$.

The functions $\chi_{p}$ in $(3.32)$ lie in $\mathscr{H}\left(G_{\mathbf{C}}\right)$. For $p \in \mathbf{N}$, if $g \in G_{\mathbf{C}}$, we have the obvious identity,

$$
|\underline{\beta}|^{2} \chi_{p}(g)=\operatorname{Tr}^{H^{(0,0)}\left(N, L^{p}\right)}\left[g \rho_{p}|\underline{\beta}|^{2}\right] .
$$

By (1.14), since $-|i \underline{\beta}|^{2}$ is a nonnegative second-order differential operator on $U$, for $t \geqslant 0$, the action of the operator $\exp \left(-t|\underline{\beta}|^{2}\right)$ on $\chi_{p}$ is well defined. By (3.36), we get

$$
\exp \left(-t|\underline{\beta}|^{2}\right) \chi_{p}(g)=\operatorname{Tr}^{H^{(0,0)}\left(N, L^{p}\right)}\left[g \exp \left(-t \rho_{p}|\underline{\beta}|^{2}\right)\right]
$$

Theorem 3.7. If $\beta$ is nondegenerate, there exist $C>0, C^{\prime}>0$ such that, for $p \in \mathbf{N}$ large enough, for $g \in U, t \geqslant 0$,

$$
\left|\exp \left(-\frac{t}{p^{2}}|\underline{\beta}|^{2}\right) \chi_{p}(g)\right| \leqslant C p^{n} \exp \left(-C^{\prime} t\right) .
$$

If $\beta$ is nondegenerate, there exist $c>0, C>0, C^{\prime}>0$ such that, for $p \in \mathbf{N}$ large enough, for $A \in \mathfrak{g}_{\mathbf{C}}, t \geqslant 0$,

$$
\left|\delta_{1 / p} \exp \left(-\frac{t}{p^{2}}|\underline{\beta}|^{2}\right) \chi_{p}(A)\right| \leqslant C p^{n} \exp \left(c|A|-C^{\prime} t\right) .
$$

Proof. If $g \in U, g$ acts as a unitary operator on $H^{(0,0)}\left(N, L^{p}\right)$. If $\beta$ is nondegenerate, by equation (3.27) in Theorem 3.3, and by (3.37), we get

$$
\left|\exp \left(-\frac{t}{p^{2}}|\underline{\beta}|^{2}\right) \chi_{p}(g)\right| \leqslant \exp \left(-\left(c-C / p^{2}\right) t\right) \chi_{p}(1) .
$$

By $(3.35)$, as $p \rightarrow+\infty$,

$$
\chi_{p}(1)=\mathcal{O}\left(p^{n}\right)
$$

By (3.40), (3.41), we get (3.38).

Take $A \in \mathfrak{g}_{\mathbf{C}}$. By equation (3.17) in Theorem 3.1, we get

$$
\left|e^{A / p}\right|_{H^{(0,0)}\left(N, L^{p}\right)} \mid \leqslant \exp (c|A|) .
$$

By proceeding as before, we get (3.39). The proof of our theorem is completed. 
Remark 3.8. For $\epsilon>0$, let $B^{\mathfrak{u}}(0, \epsilon)$ be the open ball of centre 0 and radius $\epsilon$ in $\mathfrak{u}$. For $\epsilon>0$ small enough, the exponential map identifies $B^{\mathfrak{u}}(0, \epsilon)$ with an open neighbourhood of 1 in $U$. As in $\S 1.8$, for $\epsilon>0$ small enough, we can view $|\beta|^{2}=-|i \beta|^{2}$ as a second-order differential operator acting on $C^{\infty}\left(B^{\mathfrak{u}}(0, \epsilon), \mathbf{C}\right)$.

Set

$$
|\underline{\beta}|_{p}^{2}=\delta_{1 / p} \frac{|\underline{\beta}|^{2}}{p^{2}} \delta_{p} .
$$

By proceeding as in $\S 1.8$, we find that, as $p \rightarrow+\infty$,

$$
\underline{|\beta|_{p}^{2}} \rightarrow|\beta|^{2} \text {. }
$$

Also,

$$
\delta_{1 / p} \exp \left(-\frac{t}{p^{2}}|\underline{\beta}|^{2}\right) \chi_{p}=\exp \left(-t|\underline{\beta}|_{p}^{2}\right)\left(\delta_{1 / p} \chi_{p}\right) .
$$

By (3.44), as $p \rightarrow+\infty$,

$$
\exp \left(-t|\underline{\beta}|_{p}^{2}\right) \rightarrow \exp \left(-t|\beta|^{2}\right)
$$

By (3.35), (3.46), as $p \rightarrow+\infty$,

$$
p^{-n} \exp \left(-t|\underline{\beta}|_{p}^{2}\right)\left(\delta_{1 / p} \chi_{p}\right)(A) \rightarrow \exp \left(-t|\beta|^{2}\right) R(A) .
$$

By (3.39), (3.47), if $\beta$ is nondegenerate, we get

$$
\left|\exp \left(-t|\beta|^{2}\right) R(A)\right| \leqslant C \exp \left(c|A|-C^{\prime} t\right) .
$$

Equation (3.48) fits nicely with (1.27).

\section{A lower bound for the Hodge Laplacian}

We make the same assumptions as in $\S \S 2$ and 3 , with $S$ reduced to a point. The purpose of this section is to obtain a lower bound as $p \rightarrow+\infty$ for the Hodge Laplacian $\square_{p}^{X}$ acting on $\Omega^{\cdot}\left(X, F_{p}\right)$, with $F_{p}=H^{(0,0)}\left(N, L^{p}\right)$ when $\widehat{\theta}^{\mathfrak{p}}$ is nondegenerate. The method of proof consists in expressing a part of $\square_{p}^{X}$ as a Toeplitz operator, to which the results of $\S 3$ can be applied.

This section is organized as follows. In $\S 4.1$, we construct the flat bundle $F=$ $H^{(0,0)}(N, L)$ on $X$.

In $\S 4.2$, we give a formula for the associated Hodge-de Rham Laplacian.

Finally, in $\S 4.3$, when $\widehat{\theta}^{\mathfrak{p}}$ is nondegenerate, we give a lower bound for $\square_{p}^{X}$ as $p \rightarrow+\infty$.

\subsection{A flat vector bundle}

We make the same assumptions as in $\S \S 2$ and 3 .

Recall that the manifold $\mathcal{N}$ was defined in (1.82), and fibres over $M$ with fibre $N$. We denote by $\rho$ the representation $G_{\mathbf{C}} \rightarrow \operatorname{Aut}\left[H^{(0,0)}(N, L)\right]$. Since $K, U, G, G_{\mathbf{C}}$ also act on the line bundle $L$ on $N, L$ induces a corresponding line bundle on $\mathcal{N}$, which we also denote by $L$.

Set

$$
F=P_{K} \times_{K} H^{(0,0)}(N, L)
$$


In (4.1), we may as well replace $K$ by $U, G, G_{\mathbf{C}}$. Then $F$ can be identified with the Dolbeault cohomology of $L$ along the fibres $N$. Also, $F$ is a Hermitian vector bundle on $M$. The connection $\theta^{\mathfrak{g}}$ induces a flat connection $\nabla^{F}$ on $F$, and the connection $\theta^{\mathfrak{k}}$ induces a corresponding unitary connection $\nabla^{F, u}$ on $F$. Recall that $\theta^{\mathfrak{p}}$ is a section of $T^{*} M \otimes \mathfrak{p}_{r}$. By (1.37),

$$
\nabla^{F, u}=\nabla^{F}-\rho \theta^{\mathfrak{p}} .
$$

Note that $\rho \theta^{\mathfrak{p}}$ is a 1 -form on $M$ taking its values in self-adjoint elements of $\operatorname{End}(F)$. It will be convenient to use the notation

$$
\omega\left(\nabla^{F}, g^{F}\right)=-2 \rho \theta^{\mathfrak{p}}
$$

By (4.2),

$$
\omega\left(\nabla^{F}, g^{F}\right)=\left(g^{F}\right)^{-1} \nabla^{F} g^{F} .
$$

Let $R^{F, u}$ be the curvature of $\nabla^{F, u}$. By (1.38), (4.3), we get

$$
R^{F, u}=-\rho \theta^{\mathfrak{p}, 2}=-\frac{1}{4} \omega\left(\nabla^{F}, g^{F}\right)^{2} .
$$

Let $\left(\Omega\left(X,\left.F\right|_{X}\right), d^{X}\right)$ be the fibrewise de Rham complex of smooth forms along the fibres $X$ with values in $\left.F\right|_{X}$, equipped with the fibrewise de Rham operator $d^{X}$. Then $\left(\Omega \cdot\left(X,\left.F\right|_{X}\right), d^{X}\right)$ can be viewed as a family of infinite-dimensional complexes on $S$. Let $H^{\cdot}\left(X,\left.F\right|_{X}\right)$ be the cohomology of the complex $\left(\Omega\left(X,\left.F\right|_{X}\right), d^{X}\right)$. Then $H^{\cdot}\left(X,\left.F\right|_{X}\right)$ is a finite-dimensional $\mathbf{Z}$-graded complex vector bundle on $S$.

Recall that $d v_{X}$ is the volume form along $X$. We equip $\Omega\left(X,\left.F\right|_{X}\right)$ with the metric $g^{\Omega \cdot\left(X,\left.F\right|_{X}\right)}$ associated with the $L_{2}$ Hermitian product

$$
\left\langle s, s^{\prime}\right\rangle=\int_{X}\left\langle s, s^{\prime}\right\rangle_{\Lambda^{\cdot}\left(T^{*} X\right) \otimes_{\mathbf{R}} F} d v_{X} .
$$

Let $d^{X, *}$ be the fibrewise adjoint of $d^{X}$ with respect to the Hermitian product (4.6). Set

$$
D^{X}=d^{X}+d^{X, *} \text {. }
$$

By Hodge theory,

$$
\operatorname{ker} D^{X} \simeq H^{\cdot}\left(X,\left.F\right|_{X}\right) .
$$

Moreover, if $\square^{X}$ is the Hodge Laplacian, we get

$$
D^{X, 2}=\left[d^{X}, d^{X, *}\right]=\square^{X}
$$

\subsection{A formula for the Hodge Laplacian}

Here we assume that $S$ is just a point, so that $M$ consists of a single fibre $X$.

Let $\nabla^{T X}$ be the Levi-Civita connection on $\left(T X, g^{T X}\right)$, and let $R^{T X}$ be its curvature. Let $\nabla^{\Lambda^{*}\left(T^{*} X\right)}$ be the connection on $\Lambda^{\cdot}\left(T^{*} X\right)$ that is induced by $\nabla^{T X}$. Let $\nabla^{\Lambda^{*}\left(T^{*} X\right) \otimes \mathbf{R} F, u}$ be the unitary connection on $\Lambda^{\cdot}\left(T^{*} X\right) \otimes_{\mathbf{R}} F$ that is induced by $\nabla^{\Lambda^{*}\left(T^{*} X\right)}, \nabla^{F, u}$. Let $K^{X}$ be the scalar curvature of $X$. Let $\Delta^{X, u}$ be the Bochner Laplacian acting on $\Omega^{*}(X, F)$, which is associated with the connection $\nabla^{\Lambda^{*}\left(T^{*} X\right) \otimes_{\mathbf{R}} F, u}$.

Let $e_{1}, \ldots, e_{m}$ be an orthonormal basis of $T X$, and let $e^{1}, \ldots, e^{m}$ be the corresponding dual basis of $T^{*} X$. We use the notation of $\S 1.2$ with respect to the Euclidean vector bundle $\left(T X, g^{T X}\right)$. For the moment, the forms $\theta^{\mathfrak{p}}$ and $\widehat{\theta}^{\mathfrak{p}}$ are identified. 
Theorem 4.1. The following identities hold:

$$
\begin{aligned}
& D^{X}=\left(e^{i}-i_{e_{i}}\right) \nabla_{e_{i}}^{\Lambda \cdot\left(T^{*} X\right) \otimes_{\mathbf{R}} F}+2 i_{e_{i}} \rho \widehat{\theta}^{\mathfrak{p}}\left(e_{i}\right), \\
& D^{X}=c\left(e_{i}\right) \nabla_{e_{i}}^{\Lambda \cdot\left(T^{*} X\right) \otimes_{\mathbf{R}} F, u}+\widehat{c}\left(e_{i}\right) \rho \widehat{\theta}^{\mathfrak{p}}\left(e_{i}\right) .
\end{aligned}
$$

Moreover,

$$
\begin{aligned}
D^{X, 2}= & -\Delta^{X, u}+\frac{K^{X}}{4}-\frac{1}{8}\left\langle R^{T X}\left(e_{i}, e_{j}\right) e_{k}, e_{\ell}\right\rangle c\left(e_{i}\right) c\left(e_{j}\right) \widehat{c}\left(e_{k}\right) \widehat{c}\left(e_{\ell}\right) \\
& +\rho\left|\underline{\hat{\theta}}^{\mathfrak{p}}\right|^{2}-\frac{1}{2}\left(c\left(e_{i}\right) c\left(e_{j}\right)-\widehat{c}\left(e_{i}\right) \widehat{c}\left(e_{j}\right)\right) \rho \theta^{\mathfrak{p}, 2}\left(e_{i}, e_{j}\right) \\
& +c\left(e_{i}\right) \widehat{c}\left(e_{j}\right) \rho \nabla_{e_{i}}^{T X \otimes \mathfrak{g}_{r}, u} \widehat{\theta}^{\mathfrak{p}}\left(e_{j}\right) .
\end{aligned}
$$

Proof. This follows from (4.3), (4.4), and from [22, Theorem 4.13].

Remark 4.2. For the moment, the fact that we wrote $\widehat{\theta}^{\mathfrak{p}}$ instead of $\theta^{\mathfrak{p}}$ in some of the terms in (4.11) is a matter of taste. Still the similarity of the right-hand sides of (1.47) and (4.11) is not accidental.

\subsection{Nondegeneracy of $\widehat{\theta}^{\mathfrak{p}}$ and gap in the spectrum}

Definition 4.3. Set

$$
\begin{aligned}
\Theta= & \frac{K^{X}}{4}-\frac{1}{8}\left\langle R^{T X}\left(e_{i}, e_{j}\right) e_{k}, e_{\ell}\right\rangle c\left(e_{i}\right) c\left(e_{j}\right) \widehat{c}\left(e_{k}\right) \widehat{c}\left(e_{\ell}\right) \\
& +\left|\underline{\widehat{\theta}}^{\mathfrak{p}}\right|^{2}-\frac{1}{2}\left(c\left(e_{i}\right) c\left(e_{j}\right)-\widehat{c}\left(e_{i}\right) \widehat{c}\left(e_{j}\right)\right) \theta^{\mathfrak{p}, 2}\left(e_{i}, e_{j}\right) \\
& +c\left(e_{i}\right) \widehat{c}\left(e_{j}\right) \nabla_{e_{i}}^{T X \otimes \mathfrak{g}_{r}, u} \widehat{\theta}^{\mathfrak{p}}\left(e_{j}\right) .
\end{aligned}
$$

Then $\Theta$ is a section of $\operatorname{End}\left(\Lambda^{*}\left(T^{*} X\right)\right) \otimes U \mathfrak{g}_{r}$.

Equation (4.11) can be rewritten in the form

$$
D^{X, 2}=-\Delta^{X, u}+\rho \Theta .
$$

By (4.13), we deduce that, if $s \in \Omega^{\cdot}(X, F)$,

$$
\left\langle D^{X, 2} s, s\right\rangle \geqslant\langle\rho \Theta s, s\rangle \text {. }
$$

By Theorem 3.1, we can re-express $\rho \Theta$ as a Toeplitz operator acting on the vector bundle $\Lambda^{\cdot}\left(T^{*} X\right) \otimes_{\mathbf{R}} C^{\infty}(N, L)$ over $X$.

When replacing $L$ by $L^{p}$, we denote with an extra subscript $p$ the above objects. In particular,

$$
F_{p}=P_{K} \times_{K} H^{(0,0)}\left(N, L^{p}\right) .
$$

In what follows, we will use the notation of $\S 3$ with respect to the fibre $N$. As in (1.25), $(2.56)$, set

$$
\sigma_{\widehat{\theta}^{\mathfrak{p}}}=2 \pi\left\langle\mu, i \widehat{\theta}^{\mathfrak{p}}\right\rangle
$$


Then $\sigma_{\widehat{\theta}} \mathfrak{p}$ is a section on $\mathcal{N}$ of $q^{*} \widehat{T^{*} X}$. Recall that $\widehat{\theta}^{\mathfrak{p}}$ is said to be nondegenerate if $\sigma_{\widehat{\theta}} \mathfrak{p}$ does not vanish on $\mathcal{N}$. Equivalently, there is $a>0$ such that

$$
\left|\sigma_{\widehat{\theta} \mathfrak{p}}\right|^{2} \geqslant a \text {. }
$$

Theorem 4.4. If $\widehat{\theta}^{\mathfrak{p}}$ is nondegenerate, there exist $c>0, C>0$ such that, for $p \in \mathbf{N}$,

$$
\rho_{p} \Theta \geqslant c p^{2}-C \text {. }
$$

If $\widehat{\theta}^{\mathfrak{p}}$ is nondegenerate, there exist $c>0, C>0$ such that, for $p \in \mathbf{N}$,

$$
D_{p}^{X, 2} \geqslant c p^{2}-C \text {. }
$$

If $\widehat{\theta}^{\mathfrak{p}}$ is nondegenerate, for $p \in \mathbf{N}$ large enough, $D_{p}^{X, 2}$ is invertible; i.e. $H^{\cdot}\left(X, F_{p}\right)=0$.

Proof. If $\widehat{\theta}^{\mathfrak{p}}$ is nondegenerate, since $X$ is compact, by Theorem 3.3, there exist $c>0, C>$ 0 such that

$$
\rho_{p}\left|\widehat{\widehat{\theta}}^{\mathfrak{p}}\right|^{2} \geqslant c p^{2}-C
$$

By equation (3.17) in Theorem 3.1, and by (4.12), there exist $C^{\prime}>0, C^{\prime \prime}>0$ such that

$$
\left\|\rho_{p}\left(\Theta-\left|\underline{\widehat{\theta}}^{\mathfrak{p}}\right|^{2}\right)\right\| \leqslant C^{\prime} p+C^{\prime \prime} .
$$

By (4.20), (4.21), we get (4.18). By (4.14), (4.18), we get (4.19). The proof of our theorem is completed.

Remark 4.5. The estimate (4.19) remains valid on a Galois cover $\widehat{X}$ of $X$. This fact will be used in $\S 6.6$ and in $\S 8$.

\section{The analytic torsion forms}

The purpose of this section is to summarize the main results on the analytic torsion forms of Bismut and Lott [18], which, in degree 0, coincide with the classical Ray-Singer analytic torsion [44]. Given a proper submersion $\pi: M \rightarrow S$ with compact fibre $X$, a flat vector bundle $F$ on $M$, a horizontal subbundle $T^{H} M$ of $T M$, and metrics $g^{T X}, g^{F}$, the analytic torsion forms are even forms on $S$.

This section is organized as follows. In $\S 5.1$, we introduce odd closed forms on $M$ that are associated with the flat Hermitian vector bundle $F$.

In $\S 5.2$, we interpret the de Rham operator $d^{M}$ as a flat superconnection $A^{\prime}$ on the vector bundle $\Omega\left(X,\left.F\right|_{X}\right)$ over $S$.

In $\S 5.3$, we obtain the adjoint superconnection $A^{\prime \prime}$ to $A^{\prime}$ in the sense of [18], and also the superconnection $A=\frac{1}{2}\left(A^{\prime \prime}+A^{\prime}\right)$.

In $\S 5.4$, we replace $g^{T X}$ by $g^{T X} / t$, and we construct the corresponding superconnection $A_{t}$.

In $\S 5.5$, as in [18], we construct odd closed forms $h\left(A^{\prime}, g_{t}^{\Omega \cdot\left(X,\left.F\right|_{X}\right)}\right)$ on $S$.

In $\S 5.6$, we establish a transgression formula.

In $\S 5.7$, we construct the analytic torsion forms, and we state some of their properties.

In $\S 5.8$, we specialize the construction of $\S 5.1$ to the case where $F=H^{(0,0)}(N, L)$.

Finally, in $\S 5.9$, we state the Lichnerowicz formula of [18]. 


\subsection{The odd forms associated with a flat vector bundle}

We make the same assumptions as in $\S \S 2.1$ and 2.2 , and we use the corresponding notation. Also, $F$ denotes a complex vector bundle of dimension $k$ on $M$, and $\nabla^{F}$ is a flat connection on $F$. Also, $g^{F}$ is a Hermitian metric on $F$. Let $R$ be the GL $(k, \mathbf{C})$ bundle of frames in $F$, and let $R_{U}$ be the $\mathrm{U}(k)$-bundle of unitary frames in $F$. With $G=\mathrm{GL}(k, \mathbf{C}), K=\mathrm{U}(k)$, we are in the situation that was described in $\S \S 1.1$ and 1.6. We could as well borrow our notation from these two subsections.

As in (4.4), set

$$
\omega\left(\nabla^{F}, g^{F}\right)=\left(g^{F}\right)^{-1} \nabla^{F} g^{F} .
$$

Let $\nabla^{F, u}$ be the unitary connection on $\left(F, g^{F}\right)$,

$$
\nabla^{F, u}=\nabla^{F}+\frac{1}{2} \omega\left(\nabla^{F}, g^{F}\right) .
$$

Let $R^{F, u}$ be the curvature of $\nabla^{F, u}$. One verifies that

$$
R^{F, u}=-\frac{1}{4} \omega\left(\nabla^{F}, g^{F}\right)^{2},
$$

which fits with (4.5).

By [18, Proposition 1.3], we get

$$
\operatorname{Tr}\left[\exp \left(-R^{F, u}\right)\right]=\operatorname{dim} F .
$$

By (5.3), we can rewrite (5.4) in the form

$$
\operatorname{Tr}\left[\exp \left(\omega\left(\nabla^{F}, g^{F}\right)^{2} / 4\right)\right]=\operatorname{dim} F .
$$

Equation (5.5) is also a consequence of Proposition 1.12.

Set

$$
h(x)=x e^{x^{2}}
$$

Then

$$
h^{\prime}(x)=\left(1+2 x^{2}\right) \exp \left(x^{2}\right) .
$$

We define the action of $\varphi$ on $\Lambda^{*}\left(T^{*} M\right) \otimes_{\mathbf{R}} \mathbf{C}$ as in Definition 1.10.

Definition 5.1. Set

$$
h\left(\nabla^{F}, g^{F}\right)=(2 i \pi)^{1 / 2} \varphi \operatorname{Tr}\left[h\left(\omega\left(\nabla^{F}, g^{F}\right) / 2\right)\right] .
$$

Now, we recall results by Bismut and Lott [18, Theorems 1.8 and 1.11].

Proposition 5.2. The odd form $h\left(\nabla^{F}, g^{F}\right)$ is real and closed, and its cohomology class does not depend on $g^{F}$, and will be denoted $h\left(\nabla^{F}\right)$.

Remark 5.3. This result is also a consequence of Proposition 2.7. By a construction of Bismut and Lott [18, Theorems 1.9 and 1.11], as explained in Remark 2.8, given two metrics $g^{F}, g^{F^{\prime}}$ on $F$, there is a uniquely defined class of even forms $\widetilde{h}\left(\nabla^{F}, g^{F}, g^{F^{\prime}}\right) \in$ $\Omega \cdot(M) / d \Omega^{-1}(M)$ such that

$$
d \widetilde{h}\left(\nabla^{F}, g^{F}, g^{F^{\prime}}\right)=h\left(\nabla^{F}, g^{F^{\prime}}\right)-h\left(\nabla^{F}, g^{F}\right) .
$$


By Proposition 2.17, in degree $\geqslant 3$, the cohomology class of $h\left(\nabla^{F}, g^{F}\right)$ is rigid under deformation of the flat connection $\nabla^{F}$, which is also a result of $[15$, Theorem 2.5 and Remark 2.6]. More precisely, given a smooth path of flat connections $\ell \in[0,1] \rightarrow \nabla_{\ell}^{F}$ on $F$, one can construct an even form $\tilde{h}\left(\nabla_{\ell}^{F}, g^{F}\right)$ such that

$$
d \tilde{h}\left(\nabla_{\ell}^{F}, g^{F}\right)^{(\geqslant 2)}=\left(h\left(\nabla_{1}^{F}, g^{F}\right)-h\left(\nabla_{0}^{F}, g^{F}\right)\right)^{(\geqslant 3)} .
$$

Moreover, the class $\tilde{h}\left(\nabla_{\ell}^{F}, g^{F}\right)^{(\geqslant 4)} \in \Omega^{\cdot}(M) / d \Omega^{-1}(M)$ does not depend on the path $\ell \in$ $[0,1] \rightarrow \nabla_{\ell}^{F}$

\subsection{A flat superconnection on $\Omega \cdot\left(X,\left.F\right|_{X}\right)$}

By (2.3), we have the identification of $\mathbf{Z}$-graded vector spaces,

$$
\Omega^{\cdot}(M, F) \simeq \Omega^{\cdot}\left(S, \Omega\left(X,\left.F\right|_{X}\right)\right)
$$

We follow $[18, \S 3(\mathrm{~b})]$. Let $d^{M}$ be the de Rham operator acting on $\Omega(M, F)$. By $(5.11)$, $A^{\prime}=d^{M}$ can be considered as a flat superconnection of $\Omega\left(X,\left.F\right|_{X}\right)$.

Definition 5.4. Let $\nabla^{\Omega \cdot\left(X,\left.F\right|_{X}\right)}$ be the connection on $\Omega\left(X,\left.F\right|_{X}\right)$ such that, if $U \in T S$ and if $s$ is a smooth section of $\Omega \cdot\left(X,\left.F\right|_{X}\right)$, then

$$
\nabla_{U}^{\Omega \cdot\left(X,\left.F\right|_{X}\right)} s=L_{U^{H}} s .
$$

The connection $\nabla^{\Omega^{\cdot}\left(X,\left.F\right|_{X}\right)}$ preserves the $\mathbf{Z}$-grading of $\Omega^{\cdot}\left(X,\left.F\right|_{X}\right)$.

Recall that $T^{H}$ was defined in Definition 2.1. The interior multiplication $i_{T^{H}}$ acts naturally on $\Lambda^{*}\left(T^{*} M\right) \otimes_{\mathbf{R}} F$. We have the simple result of [18, Proposition 3.4].

Proposition 5.5. The following identity of operators acting on $\Omega(M, F)$ holds:

$$
A^{\prime}=d^{X}+\nabla^{\Omega \cdot\left(X,\left.F\right|_{X}\right)}+i_{T^{H}}
$$

\subsection{The adjoint superconnection}

Let $\nabla^{\Lambda^{*}\left(T^{*} X\right) \widehat{\otimes}_{\mathbf{R}} F, u}$ be the unitary connection on $\Lambda^{\prime}\left(T^{*} X\right) \widehat{\otimes}_{\mathbf{R}} F$ induced by $\nabla^{T X}$ and $\nabla^{F, u}$. We equip the vector bundle $\Omega\left(X,\left.F\right|_{X}\right)$ with the metric $g^{\Omega \cdot\left(X,\left.F\right|_{X}\right)}$ defined as in (4.6).

Let $A^{\prime \prime}$ be the adjoint of the superconnection $A^{\prime}$ with respect to the metric $g^{\Omega^{\prime}\left(X,\left.F\right|_{X}\right)}$ in the sense of $[18, \S 1(\mathrm{~d})]$. The adjoint $d^{X, *}$ is just the fibrewise adjoint of $d^{X}$. Let $\nabla^{\Omega^{\prime}\left(X,\left.F\right|_{X}\right), *}$ be the connection on $\Omega\left(X,\left.F\right|_{X}\right)$ which is adjoint to $\nabla^{\Omega^{*}\left(X,\left.F\right|_{X}\right)}$ with respect to $g^{\Omega \cdot\left(X,\left.F\right|_{X}\right)}$. Since $T X$ and $T^{*} X$ are identified by $g^{T X}$, we can consider $T^{H}$ as a section of $\Lambda^{2}\left(T^{*} S\right) \widehat{\otimes} T^{*} X$. Then $T^{H} \wedge$ acts on $\Lambda^{\cdot}\left(T^{*} S\right) \widehat{\otimes}_{\mathbf{R}} \Omega^{\cdot}\left(X,\left.F\right|_{X}\right)$.

Then we have the result stated in [18, Proposition 3.7].

Proposition 5.6. The following identity holds:

$$
A^{\prime \prime}=d^{X, *}+\nabla^{\Omega \cdot\left(X,\left.F\right|_{X}\right), *}-T^{H} \wedge .
$$


Set

$$
A=\frac{1}{2}\left(A^{\prime \prime}+A^{\prime}\right), \quad B=\frac{1}{2}\left(A^{\prime \prime}-A^{\prime}\right) .
$$

Then $A$ is a superconnection on $\Omega\left(X,\left.F\right|_{X}\right)$, and $B$ is a smooth section of

$$
\left(\Lambda^{\cdot}\left(T^{*} S\right) \widehat{\otimes}_{\mathbf{R}} \operatorname{End}\left(\Omega \cdot\left(X,\left.F\right|_{X}\right)\right)\right)^{\text {odd }} .
$$

\subsection{A rescaling of the metric on $T X$}

For $a \in \mathbf{R}$, let $\psi_{a}$ be the endomorphism of $\Lambda^{*}\left(T^{*} S\right)$ that is defined as in (1.51). Here, $\psi_{a}$ only acts on $\Lambda^{\cdot}\left(T^{*} S\right)$ and not on $\Lambda^{*}\left(T^{*} M\right)$.

For $t>0$, set

$$
g_{t}^{T X}=\frac{g^{T X}}{t} .
$$

Let $g_{t}^{\Omega \cdot\left(X,\left.F\right|_{X}\right)}$ be the metric on $\Omega \cdot\left(X,\left.F\right|_{X}\right)$ associated to $g_{t}^{T X}$.

Recall that $m=\operatorname{dim} X$. Let $N^{\Lambda^{\prime}\left(T^{*} X\right)}$ be the number operator of $\Lambda^{*}\left(T^{*} X\right)$. Then $N^{\Lambda^{*}\left(T^{*} X\right)}$ acts by multiplication by $k$ on $\Omega^{k}\left(X,\left.F\right|_{X}\right)$. One verifies easily that

$$
g_{t}^{\Omega \cdot\left(X,\left.F\right|_{X}\right)}=t^{N^{\Lambda^{\cdot}\left(T^{*} X\right)}-m / 2} g^{\Omega \cdot\left(X,\left.F\right|_{X}\right)} .
$$

Let $A_{t}^{\prime \prime}$ be the adjoint of $A^{\prime}$ with respect to $g_{t}^{\Omega \cdot\left(X,\left.F\right|_{X}\right)}$. Clearly $A^{\prime \prime}=A_{1}^{\prime \prime}$. Moreover,

$$
A_{t}^{\prime \prime}=t^{-N^{\Lambda^{*}\left(T^{*} X\right)}} A^{\prime \prime} t^{N^{\Lambda^{*}\left(T^{*} X\right)}} .
$$

Set

$$
A_{t}=\frac{1}{2}\left(A_{t}^{\prime \prime}+A^{\prime}\right), \quad B_{t}=\frac{1}{2}\left(A_{t}^{\prime \prime}-A^{\prime}\right) .
$$

Definition 5.7. For $t>0$, set

$$
C_{t}^{\prime}=t^{N^{\Lambda^{*}\left(T^{*} X\right)} / 2} A^{\prime} t^{-N^{\Lambda^{*}\left(T^{*} X\right)} / 2}, \quad C_{t}^{\prime \prime}=t^{-N^{\Lambda^{*}\left(T^{*} X\right)} / 2} A^{\prime \prime} t^{N^{\Lambda^{*}\left(T^{*} X\right)} / 2} .
$$

Then $C_{t}^{\prime}$ is a flat superconnection on $\Omega^{\cdot}\left(X,\left.F\right|_{X}\right)$, and $C_{t}^{\prime \prime}$ is its adjoint with respect to $g^{\Omega \cdot\left(X,\left.F\right|_{X}\right)}$. Set

$$
C_{t}=\frac{1}{2}\left(C_{t}^{\prime \prime}+C_{t}^{\prime}\right), \quad D_{t}=\frac{1}{2}\left(C_{t}^{\prime \prime}-C_{t}^{\prime}\right)
$$

Clearly,

$$
C_{t}=t^{N^{\Lambda^{\cdot}\left(T^{*} X\right)} / 2} A_{t} t^{-N^{\Lambda^{\cdot}\left(T^{*} X\right)} / 2}, \quad D_{t}=t^{N^{\Lambda^{\cdot}\left(T^{*} X\right)} / 2} B_{t} t^{-N^{\Lambda^{*}\left(T^{*} X\right)} / 2} .
$$

Proposition 5.8. For $t>0$, the following identities hold:

$$
C_{t}=\psi_{\sqrt{t}}^{-1} \sqrt{t} A \psi_{\sqrt{t}}, \quad D_{t}=\psi_{\sqrt{t}}^{-1} \sqrt{t} B \psi_{\sqrt{t}} .
$$

Proof. Our proposition follows easily from the fact that $A^{\prime}=d^{M}$ increases by 1 the total degree of the forms on $M$. 
5.5. The odd closed forms $h\left(A^{\prime}, g_{t}^{\Omega \cdot\left(X,\left.F\right|_{X}\right)}\right)$

Let $\chi(X, F)$ be the Euler characteristic of $\left.F\right|_{X}$; i.e.,

$$
\chi(X, F)=\sum_{j=0}^{m}(-1)^{j} \operatorname{dim} H^{j}\left(X,\left.F\right|_{X}\right) .
$$

By the Chern-Gauss-Bonnet theorem,

$$
\chi(X, F)=\operatorname{dim} F \int_{X} e(T X) .
$$

First, we state a result established in [18, Theorem 3.15].

Proposition 5.9. For any $t>0$,

$$
\operatorname{Tr}_{\mathrm{s}}\left[\exp \left(-A_{t}^{2}\right)\right]=\chi(X, F) .
$$

We define the action of $\varphi$ on $\Lambda^{\cdot}\left(T^{*} S\right) \otimes_{\mathbf{R}} \mathbf{C}$ as in Definition 1.10.

Definition 5.10. For $t>0$, set

$$
\begin{aligned}
& h\left(A^{\prime}, g_{t}^{\Omega \cdot\left(X,\left.F\right|_{X}\right)}\right)=(2 i \pi)^{1 / 2} \varphi \operatorname{Tr}_{\mathrm{S}}\left[h\left(B_{t}\right)\right], \\
& h\left(C_{t}^{\prime}, g^{\Omega\left(X,\left.F\right|_{X}\right)}\right)=(2 i \pi)^{1 / 2} \varphi \operatorname{Tr}_{\mathrm{s}}\left[h\left(D_{t}\right)\right] .
\end{aligned}
$$

By (5.22),

$$
h\left(A^{\prime}, g_{t}^{\Omega \cdot\left(X,\left.F\right|_{X}\right)}\right)=h\left(C_{t}^{\prime}, g^{\Omega \cdot\left(X,\left.F\right|_{X}\right)}\right)
$$

The Z-graded vector bundle $H^{\cdot}\left(X,\left.F\right|_{X}\right)$ on $S$ is equipped with the flat Gauss-Manin connection $\nabla^{H^{\cdot}\left(X,\left.F\right|_{X}\right)}$. By identifying $H^{\cdot}\left(X,\left.F\right|_{X}\right)$ with the fibrewise harmonic forms in $\Omega^{\cdot}\left(X,\left.F\right|_{X}\right), H^{\cdot}\left(X,\left.F\right|_{X}\right)$ inherits a Hermitian metric $g^{H^{\cdot}\left(X,\left.F\right|_{X}\right)}$ from the metric $g^{\Omega^{\cdot}\left(X,\left.F\right|_{X}\right)}$. Set

$$
h\left(\nabla^{H^{\cdot}\left(X,\left.F\right|_{X}\right)}, g^{H^{\cdot}\left(X,\left.F\right|_{X}\right)}\right)=(2 i \pi)^{1 / 2} \varphi \operatorname{Tr}_{\mathrm{S}}\left[h\left(\omega\left(H^{\cdot}\left(X,\left.F\right|_{X}\right), g^{H^{\cdot}\left(X,\left.F\right|_{X}\right)}\right) / 2\right)\right] .
$$

We state a result that was established in [18, Theorem 3.16].

Theorem 5.11. The forms $h\left(A^{\prime}, g_{t}^{\Omega \cdot\left(X,\left.F\right|_{X}\right)}\right)$ are real, odd, closed, and their cohomology class does not depend on $t>0$. Moreover, as $t \rightarrow 0$,

$$
h\left(A^{\prime}, g_{t}^{\Omega \cdot\left(X,\left.F\right|_{X}\right)}\right)=\pi_{*}\left[e\left(T X, \nabla^{T X}\right) h\left(\nabla^{F}, g^{F}\right)\right]+\mathcal{O}(\sqrt{t}) .
$$

As $t \rightarrow+\infty$,

$$
h\left(A^{\prime}, g_{t}^{\Omega \cdot\left(X,\left.F\right|_{X}\right)}\right)=h\left(\nabla^{H^{\cdot}\left(X,\left.F\right|_{X}\right)}, g^{H^{\cdot}\left(X,\left.F\right|_{X}\right)}\right)+\mathcal{O}(1 / \sqrt{t}) .
$$

The following identity holds:

$$
h\left(\nabla^{H \cdot\left(X,\left.F\right|_{X}\right)}\right)=\pi_{*}\left[e(T X) h\left(\nabla^{F}\right)\right] \quad \text { in } H^{\text {odd }}(S, \mathbf{R}) .
$$




\subsection{A transgression formula}

Definition 5.12. For $t>0$, set

$$
\begin{aligned}
& h^{\wedge}\left(A^{\prime}, g_{t}^{\Omega \cdot\left(X,\left.F\right|_{X}\right)}\right)=\varphi \operatorname{Tr}_{\mathrm{s}}\left[\left(\frac{N^{\Lambda^{*}\left(T^{*} X\right)}}{2}-\frac{m}{4}\right) h^{\prime}\left(B_{t}\right)\right], \\
& h^{\wedge}\left(C_{t}^{\prime}, g^{\Omega^{\cdot}\left(X,\left.F\right|_{X}\right)}\right)=\varphi \operatorname{Tr}_{\mathrm{s}}\left[\left(\frac{N^{\Lambda^{\cdot}\left(T^{*} X\right)}}{2}-\frac{m}{4}\right) h^{\prime}\left(D_{t}\right)\right] .
\end{aligned}
$$

The forms in (5.33) are still real.

By (5.22),

$$
h^{\wedge}\left(A^{\prime}, g_{t}^{\Omega \cdot\left(X,\left.F\right|_{X}\right)}\right)=h^{\wedge}\left(C_{t}^{\prime}, g^{\Omega \cdot\left(X,\left.F\right|_{X}\right)}\right) .
$$

By Proposition 5.8, we get

$$
\begin{aligned}
& h\left(A^{\prime}, g_{t}^{\Omega \cdot\left(X,\left.F\right|_{X}\right)}\right)=\psi_{\sqrt{t}}^{-1}(2 i \pi)^{1 / 2} \varphi \operatorname{Tr}_{\mathrm{s}}[h(\sqrt{t} B)], \\
& h^{\wedge}\left(A^{\prime}, g_{t}^{\Omega \cdot\left(X,\left.F\right|_{X}\right)}\right)=\psi_{\sqrt{t}}^{-1} \varphi \operatorname{Tr}_{\mathrm{S}}\left[\left(\frac{N^{\Lambda^{\cdot}\left(T^{*} X\right)}}{2}-\frac{m}{4}\right) h^{\prime}(\sqrt{t} B)\right] .
\end{aligned}
$$

We have the following result established in [18, Theorem 3.20].

Theorem 5.13. The form $h^{\wedge}\left(A^{\prime}, g_{t}^{\Omega^{\cdot}\left(X,\left.F\right|_{X}\right)}\right)$ is even. Moreover,

$$
\frac{\partial}{\partial t} h\left(A^{\prime}, g_{t}^{\Omega \cdot\left(X,\left.F\right|_{X}\right)}\right)=\frac{1}{t} d h^{\wedge}\left(A^{\prime}, g_{t}^{\Omega \cdot\left(X,\left.F\right|_{X}\right)}\right) .
$$

Put

$$
\chi^{\prime}(X, F)=\sum_{j=0}^{m}(-1)^{j} j \operatorname{dim} H^{j}\left(X,\left.F\right|_{X}\right) .
$$

We recall the result of [18, Theorem 3.21].

Theorem 5.14. As $t \rightarrow 0$,

$$
h^{\wedge}\left(A^{\prime}, g_{t}^{\Omega \cdot\left(X,\left.F\right|_{X}\right)}\right)=\mathcal{O}(\sqrt{t})
$$

As $t \rightarrow+\infty$,

$$
h^{\wedge}\left(A^{\prime}, g_{t}^{\Omega^{\cdot}\left(X,\left.F\right|_{X}\right)}\right)=\left(\frac{1}{2} \chi^{\prime}(X, F)-\frac{m}{4} \chi(X, F)\right) h^{\prime}(0)+\mathcal{O}(1 / \sqrt{t}) .
$$

Now, we enlarge $M, S$ to $M \times \mathbf{R}_{+}^{*}, S \times \mathbf{R}_{+}^{*}$, and, over $M \times\{s\}$, we equip $T X$ with the metric $g^{T X} / s$. Over $M \times \mathbf{R}_{+}^{*}$, the canonical connection $\widetilde{\nabla}^{T X}$ on $T X$ is given by

$$
\widetilde{\nabla}^{T X}=\nabla^{T X}+d s\left(\frac{\partial}{\partial s}-\frac{1}{2 s}\right)
$$


By equation (2.12), for $U \in T X$,

$$
T\left(\frac{\partial}{\partial s}, U\right)=-\frac{U}{2 s} .
$$

By (2.9), if $U, V \in T X$,

$$
\left\langle S(U) V, \frac{\partial}{\partial s}\right\rangle=\frac{1}{2 s^{2}}\langle U, V\rangle .
$$

Now, we proceed as in $[18, \S 3(\mathrm{i})]$. Let $\widetilde{A}^{\prime}, \widetilde{B}_{t}$ be the analogues of $A^{\prime}, B_{t}$ over $S \times \mathbf{R}_{+}$. By $[18$, equations $(3.112)$ and $(3.113)]$, we get

$$
\widetilde{B}_{t}=B_{s t}+\frac{d s}{s}\left(\frac{N^{\Lambda^{*}\left(T^{*} X\right)}}{2}-\frac{m}{4}\right) .
$$

Let $\widetilde{g}_{t}^{\Omega \cdot\left(X,\left.F\right|_{X}\right)}$ be the analogue of $g_{t}^{\Omega \cdot\left(X,\left.F\right|_{X}\right)}$. By (5.27), (5.43), we get an equation already established in [18, equation (3.114)],

$$
h\left(\widetilde{A}^{\prime}, \widetilde{g}_{t}^{\Omega \cdot\left(X,\left.F\right|_{X}\right)}\right)=h\left(A^{\prime}, g_{s t}^{\Omega \cdot\left(X,\left.F\right|_{X}\right)}\right)+\frac{d s}{s} h^{\wedge}\left(A^{\prime}, g_{s t}^{\Omega \cdot\left(X,\left.F\right|_{X}\right)}\right) .
$$

Since the form in (5.44) in closed on $S \times \mathbf{R}_{+}^{*}$, we recover (5.36). Similarly, Theorem 5.14 can be viewed as a consequence of Theorem 5.11 and of (5.44).

\subsection{The analytic torsion forms}

Now, we follow $[18, \S 3(\mathrm{j})]$.

Definition 5.15. Set

$$
\begin{aligned}
\mathcal{T}_{h}\left(T^{H} M, g^{T X}, \nabla^{F}, g^{F}\right)= & -\int_{0}^{+\infty}\left[h^{\wedge}\left(A^{\prime}, g_{t}^{\Omega \cdot\left(X,\left.F\right|_{X}\right)}\right)\right. \\
& \left.+\left(\frac{m}{4} \chi(X, F)-\frac{1}{2} \chi^{\prime}(X, F)\right)\left(h^{\prime}(0)-h^{\prime}(i \sqrt{t} / 2)\right)\right] \frac{d t}{t} .
\end{aligned}
$$

The forms $\mathcal{T}_{h}\left(T^{H} M, g^{T X}, \nabla^{F}, g^{F}\right)$ are called analytic torsion forms.

The following result was established in [18, Theorem 3.23].

Theorem 5.16. The form $\mathcal{T}_{h}\left(T^{H} M, g^{T X}, \nabla^{F}, g^{F}\right)$ is even. Moreover,

$$
\begin{aligned}
d \mathcal{T}_{h}\left(T^{H} M, g^{T X}, \nabla^{F}, g^{F}\right)= & \pi_{*}\left[e\left(T X, \nabla^{T X}\right) h\left(\nabla^{F}, g^{F}\right)\right] \\
& -h\left(\nabla^{H \cdot\left(X,\left.F\right|_{X}\right)}, g^{H^{\cdot}\left(X,\left.F\right|_{X}\right)}\right) .
\end{aligned}
$$

Proof. Our theorem follows from Theorems 5.13 and 5.14.

Let $T^{H \prime} M, g^{T X^{\prime}}, g^{F,}$ be another triple of data. We will denote with an extra prime the objects canonically attached to this new triple. Let $\widetilde{e}\left(T X, \nabla^{T X}, \nabla^{T X^{\prime}}\right) \in$ $\Omega^{\cdot}(M) / d \Omega^{-1}(M)$ be the corresponding Chern-Simons class, so that

$$
d \widetilde{e}\left(T X, \nabla^{T X}, \nabla^{T X^{\prime}}\right)=e\left(T X, \nabla^{T X^{\prime}}\right)-e\left(T X, \nabla^{T X}\right) .
$$


The following result was established in [18, Theorem 3.24] as a consequence of Theorem 5.16.

Theorem 5.17. The following identity holds:

$$
\begin{aligned}
\mathcal{T}_{h}\left(T^{H \prime} M, g^{T X^{\prime}}, \nabla^{F}, g^{F^{\prime}}\right)-\mathcal{T}_{h}\left(T^{H} M, g^{T X}, \nabla^{F}, g^{F}\right) \\
=\pi_{*}\left[\widetilde{e}\left(T X, \nabla^{T X}, \nabla^{T X^{\prime}}\right) h\left(\nabla^{F}, g^{F}\right)\right]+\pi_{*}\left[e\left(T X, \nabla^{T X^{\prime}}\right) \widetilde{h}\left(\nabla^{F}, g^{F}, g^{F^{\prime}}\right)\right] \\
\quad-\widetilde{h}\left(\nabla^{H^{\prime}\left(X,\left.F\right|_{X}\right)}, g^{H^{\prime}(X, F \mid X)}, g^{H^{\prime}\left(X,\left.F\right|_{X} ^{\prime}\right)}\right) \quad \text { in } \Omega(S) / d \Omega^{-1}(S) .
\end{aligned}
$$

Remark 5.18. Let $\left(D^{X, 2}\right)^{-1}$ be the inverse of $D^{X, 2}$ acting on the orthogonal bundle to $\operatorname{ker} D^{X}$ in $\Omega \cdot\left(X,\left.F\right|_{X}\right)$. For $s \in \mathbf{C}, \operatorname{Re}(s)>m / 2$, set

$$
\vartheta(s)=-\operatorname{Tr}_{\mathrm{s}}\left[\left(N^{\Lambda^{*}\left(T^{*} X\right)}-\frac{m}{2}\right)\left(D^{X, 2}\right)^{-s}\right] .
$$

Then $\vartheta(s)$ extends to a meromorphic function of $s \in \mathbf{C}$, which is holomorphic near $s=0$. By definition, the Ray-Singer analytic torsion [22,44] of the de Rham complex $\left(\Omega \cdot\left(X,\left.F\right|_{X}\right), d^{X}\right)$ is given by $\frac{1}{2} \frac{\partial \vartheta}{\partial s}(0)$. By Bismut and Lott [18, Theorem 3.29],

$$
\mathcal{T}_{h}\left(T^{H} M, g^{T X}, \nabla^{F}, g^{F}\right)^{(0)}=\frac{1}{2} \frac{\partial \vartheta}{\partial s}(0) .
$$

An ingredient in the proof of (5.50) is the simple fact that

$$
h^{\wedge}\left(A^{\prime}, g_{t}^{\Omega \cdot\left(X,\left.F\right|_{X}\right)}\right)^{(0)}=\left(1+2 t \frac{\partial}{\partial t}\right) \operatorname{Tr}_{\mathrm{s}}\left[\left(\frac{N^{\Lambda^{\cdot}\left(T^{*} X\right)}}{2}-\frac{m}{4}\right) \exp \left(-t D^{X, 2} / 4\right)\right] .
$$

As shown in [22, Theorem 7.10], as $t \rightarrow 0$,

$$
\operatorname{Tr}_{\mathrm{s}}\left[\left(\frac{N^{\Lambda^{*}\left(T^{*} X\right)}}{2}-\frac{m}{4}\right) \exp \left(-t D^{X, 2} / 4\right)\right]=\mathcal{O}(1 / \sqrt{t}) .
$$

Equation (5.38) fits with (5.51), (5.52).

Assume that $X$ is odd dimensional, and that $H^{\cdot}\left(X,\left.F\right|_{X}\right)=0$. By (5.46), the form $\mathcal{T}_{h}\left(T^{H} M, g^{T X}, \nabla^{F}, g^{F}\right)$ on $S$ is closed, and by (5.48), its cohomology class does not depend on $T^{H} M, g^{T X}, g^{F}$. It will be denoted by $\mathcal{T}_{h}\left(\nabla^{F}\right)$.

Let $\ell \in[0,1] \rightarrow \nabla_{\ell}^{F}$ be a smooth 1-parameter family of flat connections on $F$. We denote by $F_{\ell}$ the flat bundle $\left(F, \nabla_{\ell}^{F}\right)$. Recall that the forms $\widetilde{h}\left(\nabla_{\ell}^{F}, g^{F}\right)$ were described in Remark 5.3.

Assume that, for $\ell \in[0,1], H^{\cdot}\left(X,\left.F_{\ell}\right|_{X}\right)=0$. Now, we have the result of $[15$, Theorem 3.45].

Theorem 5.19. If $X$ is odd dimensional, the following identity holds:

$$
\mathcal{T}_{h}\left(\nabla_{1}^{F}\right)^{(\geqslant 2)}=\mathcal{T}_{h}\left(\nabla_{0}^{F}\right)^{(\geqslant 2)} \text { in } H^{\text {even }}(S, \mathbf{R}) .
$$

If $X$ is even dimensional, then

$$
\begin{gathered}
{\left[\mathcal{T}_{h}\left(T^{H} M, g^{T X}, \nabla_{1}^{F}, g^{F}\right)-\mathcal{T}_{h}\left(T^{H} M, g^{T X}, \nabla_{0}^{F}, g^{F}\right)\right]^{(\geqslant 2)}} \\
\quad=\pi_{*}\left[e\left(T X, \nabla^{T X}\right) \widetilde{h}\left(\nabla_{\ell}^{F}, g^{F}\right)\right] \quad \text { in } \Omega^{\cdot}(S) / d \Omega^{-1}(S) .
\end{gathered}
$$




\subsection{The odd forms associated with $H^{(0,0)}\left(N, L^{p}\right)$}

We make the same assumptions as in $\S 2$ and in $\S 4.1$, and we use the corresponding notation. In particular, the flat Hermitian vector bundle $F_{p}$ on $M$ is given by (4.15). Also, we use the notation in (4.2).

By (3.32), (4.3), and (5.8), we get

$$
h\left(\nabla^{F_{p}}, g^{F_{p}}\right)=-\chi_{p}\left[\theta^{\mathfrak{p}} \exp \left(\theta^{\mathfrak{p}, 2} / 2 i \pi\right)\right] .
$$

Let $z$ be an odd Grassmann variable, which supercommutes with $\Lambda^{*}\left(T^{*} M\right)$. If $a \in$ $\mathbf{R}[z] \widehat{\otimes} \Lambda^{*}\left(T^{*} M\right)$, we can write $a$ in the form

$$
a=b+z c, \quad b, c \in \Lambda^{\cdot}\left(T^{*} M\right) .
$$

Set

$$
a^{z}=c
$$

By (5.55), we get

$$
h\left(\nabla^{F_{p}}, g^{F_{p}}\right)=\chi_{p}\left[\exp \left(\theta^{\mathfrak{p}, 2} / 2 i \pi-z \theta^{\mathfrak{p}}\right)\right]^{z}
$$

\subsection{A Lichnerowicz formula}

Let $e_{1}, \ldots, e_{m}$ be a locally defined smooth orthonormal basis of $T X$, and let $f_{1}, \ldots, f_{r}$ be a basis of $T S$. The corresponding dual bases are denoted with upper indices.

Definition 5.20. Let ${ }^{1} \nabla^{\pi^{*}} \Lambda^{\cdot}\left(T^{*} S\right) \widehat{\otimes} \Lambda^{*}\left(T^{*} X\right)$ be the fibrewise connection on the vector bundle $\pi^{*} \Lambda^{*}\left(T^{*} S\right) \widehat{\otimes} \Lambda^{*}\left(T^{*} X\right)$,

$$
\begin{aligned}
{ }^{1} \nabla^{\pi^{*} \Lambda^{\cdot}\left(T^{*} S\right) \widehat{\otimes} \Lambda^{\cdot}\left(T^{*} X\right)}= & \nabla^{\pi^{*} \Lambda^{\cdot}\left(T^{*} S\right) \widehat{\otimes} \Lambda^{\cdot}\left(T^{*} X\right)}+\frac{1}{2}\left\langle S e_{i}, f_{\alpha}^{H}\right\rangle \sqrt{2} c\left(e_{i}\right) f^{\alpha} \\
& +\frac{1}{2}\left\langle S f_{\alpha}^{H}, f_{\beta}^{H}\right\rangle f^{\alpha} f^{\beta} .
\end{aligned}
$$

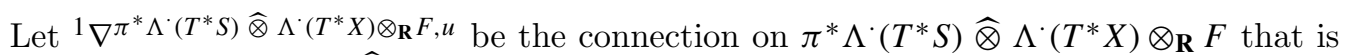
induced by ${ }^{1} \nabla^{\pi^{*} \Lambda^{*}\left(T^{*} S\right) \widehat{\otimes} \Lambda^{*}\left(T^{*} X\right)}, \nabla^{F, u}$. For $t>0$, set

$$
{ }^{1} \nabla_{t}^{\pi^{*} \Lambda^{\cdot}\left(T^{*} S\right) \widehat{\otimes} \Lambda^{\cdot}\left(T^{*} X\right) \otimes_{\mathbf{R}} F, u}=\psi_{1 / \sqrt{t}}^{1} \nabla^{\pi^{*} \Lambda^{\cdot}\left(T^{*} S\right) \widehat{\otimes} \Lambda^{\cdot}\left(T^{*} X\right) \widehat{\otimes} \mathbf{R} F, u} \psi_{\sqrt{t}} .
$$

Put

$$
\mathcal{R}=\frac{1}{4}\left\langle e_{i}, R^{T X} e_{j}\right) \widehat{c}\left(e_{i}\right) \widehat{c}\left(e_{j}\right)-\theta^{\mathfrak{p}, 2} .
$$

Then $\mathcal{R}$ is a smooth section of $\Lambda^{\prime}\left(T^{*} M\right) \widehat{\otimes} \widehat{c}(T X) \widehat{\otimes} \mathfrak{k}_{r}$.

Let $K^{X}$ be the scalar curvature of the fibres $\left(X, g^{T X}\right)$. Let $z$ be an odd Grassmann variable which anticommutes with all the other odd objects we met before. Let $\theta_{a}$ be the analogue of $\psi_{a}$ when acting on $\mathbf{R}[z] \widehat{\otimes} \Lambda^{\cdot}\left(T^{*} S\right)$. In particular,

$$
\theta_{a} z=a z
$$

Definition 5.21. For $t>0$, set

$$
\begin{aligned}
\Lambda_{t}= & t \frac{K^{X}}{16}+\frac{t}{8} c\left(e_{i}\right) c\left(e_{j}\right) \mathcal{R}\left(e_{i}, e_{j}\right)+\frac{1}{2} f^{\alpha} f^{\beta} \mathcal{R}\left(f_{\alpha}^{H}, f_{\beta}^{H}\right) \\
& +\frac{\sqrt{t}}{2} c\left(e_{i}\right) f^{\alpha} \mathcal{R}\left(e_{i}, f_{\alpha}^{H}\right)+\left.\frac{t}{4} \underline{\widehat{\theta}}^{\mathfrak{p}}\right|^{2}+\frac{\sqrt{t}}{2} f^{\alpha} \widehat{c}\left(e_{i}\right) \nabla_{f_{\alpha}^{H}}^{T X \otimes \mathfrak{g}_{r}, u} \widehat{\theta}^{\mathfrak{p}}\left(e_{i}\right)
\end{aligned}
$$




$$
\begin{aligned}
& +\frac{t}{8} \widehat{c}\left(e_{i}\right) \widehat{c}\left(e_{j}\right) \widehat{\theta}^{\mathfrak{p}, 2}\left(e_{i}, e_{j}\right)+\frac{t}{4} c\left(e_{i}\right) \widehat{c}\left(e_{j}\right) \nabla_{e_{i}}^{T X \otimes \mathfrak{g}_{r}, u} \widehat{\theta}^{\mathfrak{p}}\left(e_{j}\right) \\
& +\frac{1}{2} z \sqrt{t} c\left(e_{i}\right) \theta^{\mathfrak{p}}\left(e_{i}\right)+z f^{\alpha} \theta^{\mathfrak{p}}\left(f_{\alpha}^{H}\right) .
\end{aligned}
$$

Then $\Lambda_{t}$ is a section of $\Lambda^{*}\left(T^{*} S\right) \widehat{\otimes} c(T X) \widehat{\otimes} \widehat{c}(T X) \otimes U \mathfrak{g}_{r}$. Set

$$
\Lambda=\Lambda_{1}
$$

Then

$$
\Lambda_{t}=\theta_{\sqrt{t}}^{-1} t \Lambda \theta_{\sqrt{t}}
$$

Put

$$
L_{t}^{F}=C_{t}^{2}-z D_{t}
$$

The following formula was established by Bismut and Lott in [18, Theorem 3.11], [15, Theorem 3.19] as a consequence of the Lichnerowicz formula for the curvature of the Levi-Civita superconnection given in [18, Theorem 3.6].

Theorem 5.22. Given $t \in \mathbf{R}_{+}^{*}$, for $p \in \mathbf{N}$, the following identity holds:

$$
L_{t}^{F_{p}}=-\frac{t}{4}\left({ }^{1} \nabla_{t / 2, e_{i}}^{\pi^{*} \Lambda^{\cdot}\left(T^{*} S\right) \widehat{\otimes} \Lambda^{*}\left(T^{*} X\right) \otimes_{\mathbf{R}} F_{p}, u}-\frac{z}{\sqrt{t}} \widehat{c}\left(e_{i}\right)\right)^{2}+\rho_{p} \Lambda_{t} .
$$

\section{The asymptotics of the odd superconnection forms}

In this section, under the assumptions of $\S \S 2$ and 5 , we obtain the asymptotics as $p \rightarrow$ $+\infty$ of the odd forms $h\left(A^{\prime}, g^{\Omega^{\prime}\left(X,\left.F_{p}\right|_{X}\right)}\right)$ on $S$.

This section is organized as follows. In $\S 6.1$, we obtain the asymptotics of the forms $h\left(\nabla^{F_{p}}, g^{F_{p}}\right)$.

In $\S 6.2$, replacing the vector bundle $F$ by the infinite-dimensional vector bundle $\mathscr{F}$ of fibrewise holomorphic functions along the fibres of $P_{G_{\mathbf{C}}}$, we give a universal construction of the operators $C_{t}, D_{t}$ that were considered in $\S 5$. In this way, we obtain an operator $L_{t}^{\mathscr{F}}$.

In $\S 6.3$, we introduce the vector bundle $\mathbf{F}$ of fibrewise smooth functions along the fibres of $P_{G_{\mathbf{C}}}$, and we lift the operator $L_{t}^{\mathscr{F}}$ to ordinary differential operators $L_{t}^{\mathbf{F}}, L_{t}^{\mathbf{F} \prime}$ on the total space $\mathcal{P}_{G_{\mathbf{C}}}$ of $P_{G_{\mathbf{C}}}$ along a fibre $X$. In particular, $L_{t}^{\mathbf{F} \prime}$ is elliptic along the fibres of $\mathcal{P}_{G_{\mathbf{C}}}$.

In $\S 6.4$, we give a formula expressing the odd superconnection forms $h\left(A^{\prime}, g_{t}^{\Omega^{\prime}\left(X,\left.F\right|_{X}\right)}\right)$ in terms of a pairing of the heat kernels for $L_{t}^{\mathbf{F}}$ or $L_{t}^{\mathbf{F} \prime}$, and of the character $\chi$ associated with the action of $G_{\mathbf{C}}$ on $H^{(0,0)}(X, L)$.

In $\S 6.5$, the asymptotics of the forms $h\left(A^{\prime}, g_{t / p^{2}}^{\Omega^{\prime}\left(X, F_{p} \mid X\right)}\right)$ are obtained in terms of the forms $\pi_{*} c_{t / 4}$ that were defined in $\S 2$.

Finally, in $\S 6.6$, we briefly consider the case where $S$ is reduced to a point, and where $X$ is replaced by a Galois cover $\widehat{X}$. 


\subsection{The asymptotics of the forms $h\left(\nabla^{F_{p}}, g^{F_{p}}\right)$}

If $\left.\alpha_{p}\right|_{p \in \mathbf{N}}$ is a family of smooth differential forms on $M$, we will say that, given $k \in \mathbf{N}$, $\alpha_{p}=\mathcal{O}\left(p^{k}\right)$ as $p \rightarrow+\infty$ if, for every compact set $K \subset M, r \in \mathbf{N}$, the sup of $\left|\alpha_{p}\right|$ and its derivatives of order $\leqslant r$ over $K$ is dominated by $C_{K, r} p^{k}$.

We make the same assumptions as in $\S \S 2$ and 5 . Recall that the odd closed form $\gamma$ on $M$ was defined in Definition 2.6.

Proposition 6.1. As $p \rightarrow+\infty$,

$$
p^{-n} \frac{1}{\sqrt{p}} \psi_{1 / \sqrt{p}} h\left(\nabla^{F_{p}}, g^{F_{p}}\right)=\gamma+\mathcal{O}\left(p^{-1}\right) .
$$

Proof. By (5.58), we get

$$
\frac{1}{\sqrt{p}} \psi_{1 / \sqrt{p}} h\left(\nabla^{F_{p}}, g^{F_{p}}\right)=\left(\delta_{1 / p} \chi_{p}\right)\left(\theta^{\mathfrak{p}, 2} / 2 i \pi-z \theta^{\mathfrak{p}}\right)^{z} .
$$

By Theorem 3.6, as $p \rightarrow+\infty$,

$$
\left(\delta_{1 / p} \chi_{p}\right)\left(\theta^{\mathfrak{p}, 2} / 2 i \pi-z \theta^{\mathfrak{p}}\right)=p^{n} R\left(\theta^{\mathfrak{p}, 2} / 2 i \pi-z \theta^{\mathfrak{p}}\right)+\mathcal{O}\left(p^{n-1}\right) .
$$

By (2.52), we get

$$
R\left(\theta^{\mathfrak{p}, 2} / 2 i \pi-z \theta^{\mathfrak{p}}\right)^{z}=\gamma
$$

By (6.2)-(6.4), we get (6.1).

\subsection{The universal construction of the operators $C_{t}, D_{t}$}

Let $\mathcal{P}_{G_{\mathbf{C}}}$ denote the total space of $P_{G_{\mathbf{C}}}$ over a given fibre $X$.

Recall that $\rho_{p}$ denotes the representation $G_{\mathbf{C}} \rightarrow \operatorname{Aut}\left[H^{(0,0)}\left(N, L^{p}\right)\right]$. There is an obvious action of $G_{\mathbf{C}}$ on $C^{\infty}\left(\mathcal{P}_{G_{\mathbf{C}}}, H^{(0,0)}\left(N, L^{p}\right)\right)$ such that

$$
g s(u)=\rho_{p}(g) s(u g) .
$$

Then $C^{\infty}\left(X, F_{p}\right)$ can be identified with $C^{\infty}\left(\mathcal{P}_{G_{\mathbf{C}}}, H^{(0,0)}\left(N, L^{p}\right)\right)^{G_{\mathbf{C}}}$, the vector space of $G_{\mathbf{C}}$-invariant sections in $C^{\infty}\left(\mathcal{P}_{G_{\mathbf{C}}}, H^{(0,0)}\left(N, L^{p}\right)\right)$. Such invariant functions are holomorphic functions along the fibres of $P_{G_{\mathrm{C}}}$.

Recall that $\mathscr{H}\left(G_{\mathbf{C}}\right)$ is the vector space of complex-valued holomorphic functions on $G_{\mathbf{C}}$. By restriction to the compact group $U$, the vector space $\mathscr{H}\left(G_{\mathbf{C}}\right)$ inherits an $L_{2}$ Hermitian product. The left action of $G_{\mathbf{C}}$ on $G_{\mathbf{C}}$ induces a corresponding left action $\sigma$ of $G_{\mathbf{C}}$ on $\mathscr{H}\left(G_{\mathbf{C}}\right)$. The induced action of $U$ on $\mathscr{H}\left(G_{\mathbf{C}}\right)$ is unitary. Also, there is an associated action of $U \mathfrak{g}_{\mathbf{C}}$ on $\mathscr{H}\left(G_{\mathbf{C}}\right)$, which we still denote $\sigma$.

Set

$$
\mathscr{F}=P_{G} \times_{G} \mathscr{H}\left(G_{\mathbf{C}}\right)=P_{G_{\mathbf{C}}} \times_{G_{\mathbf{C}}} \mathscr{H}\left(G_{\mathbf{C}}\right)=P_{U} \times_{U} \mathscr{H}\left(G_{\mathbf{C}}\right) .
$$

Then $\mathscr{F}$ is the vector bundle on $M$ of the holomorphic functions along the fibres of $P_{G_{\mathbf{C}}}$. Also, $U \mathfrak{g}_{\mathbf{C}, r}$ acts on $\mathscr{F}$ as the algebra of fibrewise holomorphic right-invariant differential operators. If $A \in U \mathfrak{g}_{\mathbf{C}, r}, f \in \mathscr{F}$, the action of $A$ on $f$ will be $\operatorname{denoted} \sigma(A) f$. 
The vector bundle $\mathscr{F}$ is naturally equipped with the connections $\nabla^{\mathscr{F}}, \nabla^{\mathscr{F}}, u$ that are induced by the connections $\theta^{\mathfrak{g}}, \theta^{\mathfrak{k}}$, the connection $\nabla^{\mathscr{F}}$ is flat, and the connection $\nabla^{\mathscr{F}}, u$ is unitary. By (4.2),

$$
\nabla^{\mathscr{F}, u}=\nabla^{\mathscr{F}}-\theta^{\mathfrak{p}}
$$

By (4.5), the curvature $R^{\mathscr{F}, u}$ of $\nabla^{\mathscr{F}, u}$ is given by

$$
R^{\mathscr{F}, u}=-\theta^{\mathfrak{p}, 2} .
$$

Since $\sigma$ induces the tautological representation of $U \mathfrak{g}_{\mathbf{C}}$ on $\mathscr{H}\left(G_{\mathbf{C}}\right)$, in $(6.7)$, (6.8), we wrote $\theta^{\mathfrak{p}}, \theta^{\mathfrak{p}, 2}$ instead of $\sigma\left(\theta^{\mathfrak{p}}\right), \sigma\left(\theta^{\mathfrak{p}, 2}\right)$.

The left action of $G_{\mathbf{C}}$ on itself induces an action of $G_{\mathbf{C}}$ on $C^{\infty}\left(G_{\mathbf{C}}, \mathbf{C}\right)$, which will be still denoted $\sigma$. Also, $\mathscr{H}\left(G_{\mathbf{C}}\right) \subset C^{\infty}\left(G_{\mathbf{C}}, \mathbf{C}\right)$. Set

$$
\mathbf{F}=P_{G_{\mathbf{C}}} \times_{G_{\mathbf{C}}} C^{\infty}\left(G_{\mathbf{C}}, \mathbf{C}\right) .
$$

Then $\mathscr{F}$ embeds into $\mathbf{F}$. The connection form $\theta^{\mathfrak{k}}$ induces a connection $\nabla^{\mathbf{F}, u}$ on the vector bundle $\mathbf{F}$, whose curvature is still given by (6.8).

Let $Q$ be the $\mathrm{O}(m)$-principal bundle of orthonormal frames in $T X$, which we equip with the connection induced by $\nabla^{T X}$. Let $P_{G}^{\prime}$ denote the fibre product of $P_{G}$ with $Q$. Then $P_{G}^{\prime}$ is an $\mathrm{O}(m) \times G$ principal bundle. In this construction, we can replace $G$ by $G_{\mathbf{C}}$ or $U$. In particular, the connections $\theta^{\mathfrak{g}}, \theta^{\mathfrak{k}}$ induce corresponding connections on the above principal bundles.

In the constructions of $\S 5$, when replacing $F$ by $\mathscr{F}$, we obtain the superconnection $C_{t}^{\mathscr{F}}$ on $\Omega^{\cdot}\left(X,\left.\mathscr{F}\right|_{X}\right)$ and the operator $D_{t}^{\mathscr{F}}$. Let $L_{t}^{\mathscr{F}}$ be the analogue of $L_{t}^{F}$ in $(5.66)$, so that

$$
L_{t}^{\mathscr{F}}=C_{t}^{\mathscr{F}, 2}-z D_{t}^{\mathscr{F}} .
$$

Theorem 6.2. Given $t \in \mathbf{R}_{+}^{*}$, the following identity holds:

$$
L_{t}^{\mathscr{F}}=-\frac{t}{4}\left({ }^{1} \nabla_{t / 2, e_{i}}^{\pi * T^{*}}\left(T^{*}\right) \widehat{\otimes} \Lambda^{\cdot}\left(T^{*} X\right) \widehat{\otimes} \mathscr{F}, u-\frac{z}{\sqrt{t}} \widehat{c}\left(e_{i}\right)\right)^{2}+\Lambda_{t} .
$$

Proof. This is just a formal consequence of Theorem 5.22.

Clearly, $L_{t}^{\mathscr{F}}$ acts on $\Lambda^{*}\left(T^{*} S\right) \widehat{\otimes} \Omega^{\cdot}\left(X,\left.\mathscr{F}\right|_{X}\right)$. This is the space of smooth sections of $\Lambda^{\cdot}\left(T^{*} S\right) \widehat{\otimes} \Lambda^{\cdot}\left(T^{*} X\right)$ along the fibres $\mathcal{P}_{G_{\mathbf{C}}}$ that are holomorphic along the fibres of $P_{G_{\mathbf{C}}}$.

\subsection{Lifting $L_{t}^{\mathscr{F}}$}

Let Op be the algebra of scalar differential operators along the fibre $X$. Then $L_{t}^{\mathscr{F}}$ is a section over $S$ of

$$
\mathrm{Op} \otimes \mathbf{R}[z] \widehat{\otimes} \operatorname{End}\left(\Lambda^{\cdot}\left(T^{*} X\right)\right) \otimes_{\mathbf{R}} U \mathfrak{g}_{\mathbf{C}, r} .
$$

Let $U_{\mathbf{R}} \mathfrak{g}_{\mathbf{C}}$ be the real enveloping algebra of $\mathfrak{g}_{\mathbf{C}}$; i.e., $U_{\mathbf{R}} \mathfrak{g}_{\mathbf{C}}$ is the enveloping algebra of $G_{\mathbf{C}}$ considered as a real Lie group. Since $\mathfrak{g}_{\mathbf{C}}=\mathfrak{u}_{\mathbf{C}}, U_{\mathbf{R}} \mathfrak{g}_{\mathbf{C}}=U_{\mathbf{R}} \mathfrak{u}_{\mathbf{C}}$. Also, $U_{\mathbf{R}} \mathfrak{g}_{\mathbf{C}}$ maps into $U \mathfrak{g}_{\mathbf{C}}$. Similarly, we can construct the bundle of real enveloping algebras $U_{\mathbf{R}} \mathfrak{g}_{\mathbf{C}, r}$.

We now construct a lift $L_{t}^{\mathbf{F}}$ of $L_{t}^{\mathscr{F}}$ to

$$
\mathrm{Op} \otimes \mathbf{R}[z] \widehat{\otimes} \operatorname{End}\left(\Lambda^{\cdot}\left(T^{*} X\right)\right) \otimes U_{\mathbf{R} \mathfrak{g}, r} .
$$


Clearly, the first term in the right-hand side of (6.11) lifts naturally to an operator in (6.13).

Consider $\Lambda_{t}$ in (5.63). We proceed as in $\S \S 1.3$ and 3.5. We have the identity

$$
\left|\widehat{\widehat{\theta}}^{\mathfrak{p}}\right|^{2}=-\left|i \underline{\widehat{\theta}}^{\mathfrak{p}}\right|^{2} \quad \text { in } U \mathfrak{g}_{\mathbf{C}, r}
$$

The right-hand side of (6.14) can also be viewed as an element of $U_{\mathbf{R}} \mathfrak{g}_{\mathbf{C}, r}$, which will be our lift of $\left|\widehat{\theta}^{\mathfrak{p}}\right|^{2}$ in $U_{\mathbf{R}} \mathfrak{g}_{\mathbf{C}, r}$.

We consider another part of $\Lambda_{t}$, which, up to the factor $t$, is given by

$$
\begin{aligned}
- & \frac{1}{8}\left(c\left(e_{i}\right) c\left(e_{j}\right)-\widehat{c}\left(e_{i}\right) \widehat{c}\left(e_{j}\right)\right) \theta^{\mathfrak{p}, 2}\left(e_{i}, e_{j}\right)=\frac{1}{4}\left(e^{i} \wedge i_{e_{j}}-e^{j} \wedge i_{e_{i}}\right) \theta^{\mathfrak{p}, 2}\left(e_{i}, e_{j}\right) \\
= & -\frac{1}{4} \sum_{i<j}\left(\underline{\theta}^{\mathfrak{p}, 2}\left(e_{i}, e_{j}\right)-e^{i} \wedge i_{e_{j}}+e^{j} \wedge i_{e_{i}}\right)^{2}-\frac{1}{4} \sum_{i<j}\left[\sqrt{-1} \underline{\theta}^{\mathfrak{p}, 2}\left(e_{i}, e_{j}\right)\right]^{2} \\
& +\frac{1}{4} \sum_{i<j}\left(e^{i} \wedge i_{e_{j}}-e^{j} \wedge i_{e_{i}}\right)^{2} .
\end{aligned}
$$

We can view the right-hand side of (6.15) as a section of the bundle of algebras in (6.13), which lifts the corresponding left-hand side.

The same procedure can also be applied to the remaining terms in (5.63). Ultimately we have produced the required lift of $L_{t}^{\mathscr{F}}$ to $L_{t}^{\mathbf{F}}$. Then $L_{t}^{\mathbf{F}}$ acts naturally on $\Lambda^{\cdot}\left(T^{*} S\right) \widehat{\otimes} \Omega^{\cdot}\left(X,\left.\mathbf{F}\right|_{X}\right)$. Moreover, when acting on $\Lambda^{*}\left(T^{*} S\right) \widehat{\otimes} \Omega^{\cdot}\left(X,\left.\mathscr{F}\right|_{X}\right), L_{t}^{\mathbf{F}}$ restricts to $L_{t}^{\mathscr{F}}$. The operator $L_{t}^{\mathbf{F}}$ is a second-order operator with nonnegative scalar principal symbol, which commutes with the right action of $G_{\mathbf{C}}$ on $\mathcal{P}_{G_{\mathbf{C}}}$.

The operator $L_{t}^{\mathbf{F}}$ can be made to be elliptic. Indeed, we have the real splitting $\mathfrak{g}_{\mathbf{C}}=\mathfrak{u} \oplus$ $i \mathfrak{u}$. Also, $\mathfrak{u}$ is equipped with a $U$-invariant scalar product, which induces a corresponding $U$-invariant scalar product on $\mathfrak{g}_{\mathbf{C}}$. Consider the right-invariant Laplacian on $C^{\infty}\left(G_{\mathbf{C}}, \mathbf{C}\right)$. Since this operator commutes with the left action of $K$ on $C^{\infty}\left(G_{\mathbf{C}}, \mathbf{C}\right)$, it induces a corresponding fibrewise elliptic operator on $\mathbf{F}=P_{K} \times{ }_{K} C^{\infty}\left(G_{\mathbf{C}}, \mathbf{C}\right)$, which we denote by $\Delta^{G_{\mathbf{C}}}$. This operator is invariant under the right action of $G_{\mathbf{C}}$ on $P_{G_{\mathbf{C}}}$, and vanishes on $\mathscr{H}\left(G_{\mathbf{C}}\right)$.

By adding to $L_{t}^{\mathbf{F}}$ a multiple of $-t \Delta^{G_{\mathbf{C}}}$ which is large enough, we obtain in this way an elliptic operator $L_{t}^{\mathbf{F} \prime}$ on $\mathcal{P}_{G_{\mathbf{C}}}$ which is still a lift of $L_{t}^{\mathscr{F}}$.

We claim that, for $t>0$, the heat operators $\exp \left(-L_{t}^{\mathbf{F}}\right)$, $\exp \left(-L_{t}^{\mathbf{F} \prime}\right)$ are well defined. An easy method to construct $\exp \left(-L_{t}^{\mathbf{F}}\right)$ is via stochastic differential equations. The sum of squares in (6.15) is easily accessible to a probabilistic treatment. For more details, we refer in particular to [7, Proposition 3.7]. The fact that the fibres of $P_{G_{\mathbf{C}}}$ are noncompact does not create any difficulty, because the operators considered are invariant under the right action of $G_{\mathbf{C}}$. The operator $L_{t}^{\mathbf{F} \prime}$ being elliptic, the construction of the operator $\exp \left(-L_{t}^{\mathbf{F} \prime}\right)$ is even simpler.

The fibres of $P_{G_{\mathbf{C}}}$ are equipped with a volume form $d q$ which comes from the left-invariant Haar measure ${ }^{1}$ associated with the scalar product of $\mathfrak{g}_{\mathbf{C}}$. Then $d q$ is invariant by the right action of $G_{\mathbf{C}}$ on $P_{G_{\mathbf{C}}}$. Also, $d v_{X} d q$ is a volume form on $\mathcal{P}_{G_{\mathbf{C}}}$.

${ }^{1}$ Since $G$ is unimodular, this is also the case for $G_{\mathbf{C}}$. 
Let $P_{t}^{\mathbf{F}}\left(u, u^{\prime}\right)$ be the smooth kernel on $\mathcal{P}_{G_{\mathbf{C}}}$ which is associated with $\exp \left(-L_{t}^{\mathbf{F} \prime}\right)$ with respect to $d v_{X} d q$. In general, there is no smooth kernel associated with $\exp \left(-L_{t}^{\mathbf{F}}\right)$ but only a measure-valued kernel $P_{t}^{\mathbf{F}}\left(u, d u^{\prime}\right)$. However, because $L_{t}^{\mathbf{F}}$ is elliptic in directions which are normal to the fibres of $P_{G_{\mathbf{C}}}$, it can be written in the form

$$
P_{t}^{\mathbf{F}}\left(u, d u^{\prime}\right)=Q_{t}^{\mathbf{F}}\left(p u, p u^{\prime}\right) d v_{X} S_{t, u, p u^{\prime}}^{\mathbf{F}}(d q),
$$

where $Q_{t}^{\mathbf{F}}\left(x, x^{\prime}\right)$ is smooth on $X \times X$, and $S_{t, u, x^{\prime}}^{\mathbf{F}}(d q)$ is a measure along the fibre $P_{G_{\mathbf{C}}, x^{\prime}}$ which depends smoothly on $u, x^{\prime}$.

\subsection{A formula for $h_{t}\left(A^{\prime}, g_{t}^{\Omega^{\cdot}\left(X,\left.F\right|_{X}\right)}\right)$}

Let $P_{t}^{F}\left(x, x^{\prime}\right)$ be the smooth kernel associated with the operator $\exp \left(-L_{t}^{F}\right)$ with respect to the volume $d v_{X}\left(x^{\prime}\right)$. By (5.27), (5.66), we get

$$
h\left(A^{\prime}, g_{t}^{\Omega \cdot\left(X,\left.F\right|_{X}\right)}\right)=(2 i \pi)^{1 / 2} \varphi \int_{X} \operatorname{Tr}_{\mathrm{s}}\left[P_{t}^{F}(x, x)\right]^{z} d v_{X}(x) .
$$

We use the notation

$$
\chi=\chi_{1}
$$

If $x \in X, u, u^{\prime} \in P_{G_{\mathbf{C}}, x}$, then $u^{\prime-1} u \in G_{\mathbf{C}}$. We claim that

$$
\int_{P_{G_{\mathbf{C}}, x}} \operatorname{Tr}_{\mathrm{s}} \Lambda^{\cdot\left(T^{*} X\right)}\left[P_{t}^{\mathbf{F}}\left(u, u^{\prime}\right)\right] \chi\left(u^{\prime-1} u\right) d q\left(u^{\prime}\right)
$$

descends to a smooth function on $X$. Indeed, $\chi$ is an Ad-invariant function, and, moreover, $\exp \left(-L_{t}^{\mathbf{F} \prime}\right)$ commutes with right multiplication by $G_{\mathbf{C}}$. As to the existence of the integral in (6.19), it follows from elementary estimates.

We claim the above considerations also apply to the kernel $P_{t}^{\mathbf{F}}$. Using the notation in (6.16), and under the same assumptions as in (6.19), consider the integral

$$
\operatorname{Tr}_{\mathrm{S}}{ }^{\Lambda^{\cdot}\left(T^{*} X\right)}\left[Q_{t}^{\mathbf{F}}(x, x)\right] \int_{P_{G_{\mathbf{C}}}} \chi\left(u^{\prime-1} u\right) S_{t, u, x}^{\mathbf{F}}(d q) .
$$

Since $\exp \left(-L_{t}^{\mathbf{F}}\right)$ commutes with the right action of $G_{\mathbf{C}}$ on $P_{G_{\mathbf{C}}}$, the measure $S_{t, u, x}^{\mathbf{F}}$ on the fibre $P_{G_{\mathbf{C}}}$ comes from a measure $S_{t, x}^{\mathbf{F}}(d g)$ on $G_{\mathbf{C}, r, x}$ through the left action of $G_{\mathbf{C}, r}$ on $P_{G_{\mathbf{C}}}$, so that $(6.20)$ can be written as

$$
\operatorname{Tr}_{\mathrm{S}} \Lambda^{\Lambda^{\prime}\left(T^{*} X\right)}\left[Q_{t}^{\mathbf{F}}(x, x)\right] \int_{G_{\mathbf{C}, r, x}} \chi\left(g^{-1}\right) S_{t, x}^{\mathbf{F}}(d g) .
$$

Theorem 6.3. For $t>0$, the following identities hold:

$$
\begin{aligned}
& h\left(A^{\prime}, g_{t}^{\Omega^{\prime}\left(X,\left.F\right|_{X}\right)}\right) \\
& \quad=(2 i \pi)^{1 / 2} \varphi \int_{X}\left[\operatorname{Tr}_{\mathrm{S}} \Lambda^{\Lambda^{\prime}\left(T^{*} X\right)}\left[Q_{t}^{\mathbf{F}}(x, x)\right]^{z} \int_{G_{\mathbf{C}, r, x}} \chi\left(g^{-1}\right) S_{t, x}^{\mathbf{F}}(d g)\right] d v_{X}(x) \\
& \quad=(2 i \pi)^{1 / 2} \varphi \int_{X}\left[\int_{P_{G_{\mathbf{C}}, x}} \operatorname{Tr}_{\mathrm{s}}^{\Lambda^{\prime}\left(T^{*} X\right)}\left[P_{t}^{\mathbf{F} \prime}\left(u, u^{\prime}\right)\right]^{z} \chi\left(u^{\prime-1} u\right) d q\left(u^{\prime}\right)\right] d v_{X}(x)
\end{aligned}
$$


Proof. As explained in $\S 6.2$, smooth sections of $F$ on $X$ can be identified with $G_{\mathbf{C}}$-invariant functions on $P_{G_{\mathbf{C}}}$ under the action described in (6.5), and such sections are fibrewise holomorphic. Recall that $F$ is modelled after the vector space $H^{(0,0)}(N, L)$. The operators $L_{t}^{\mathbf{F}}$ and $L_{t}^{\mathbf{F} \prime}$ can be considered as operators acting on $C^{\infty}\left(\mathcal{P}_{G_{\mathbf{C}}}, \pi^{*} \Lambda^{*}\left(T^{*} X\right) \otimes\right.$ $H^{(0,0)}(N, L)$ ) which commute with the right action of $G_{\mathbf{C}}$. The solution of the heat equation $\exp \left(-s L_{t}^{F}\right)$ acting on $\Omega\left(X,\left.F\right|_{X}\right)$ lifts in the proper sense to the operators $\exp \left(-s L_{t}^{\mathbf{F}}\right)$ or $\exp \left(-s L_{t}^{\mathbf{F} \prime}\right)$. Equation (6.22) then becomes a tautology.

Remark 6.4. The main point of equation (6.22) is that the dependence on the representation $\rho$ is only via the character $\chi$.

\subsection{The asymptotics as $p \rightarrow+\infty$ of the odd superconnection forms}

Let $\left.\alpha_{p, t}\right|_{p \in \mathbf{N}, t>0}$ be a family of smooth differential forms on $S$. Let $k \in \mathbf{N}$. We will say that, as $p \rightarrow+\infty, \alpha_{p, t}=\mathcal{O}\left(p^{k}\right)$ if, for any compact set $K \subset S$, for $M \geqslant 0, r \in \mathbf{N}$, for $0<t \leqslant M$, the sup of $\alpha_{p, t}$ and its derivatives on $K$ is dominated by $C_{K, r} p^{k}$.

Recall that the forms $c_{t}, d_{t}$ on $M$ were defined in Definition 2.9.

Theorem 6.5. As $p \rightarrow+\infty$,

$$
\begin{aligned}
\frac{1}{\sqrt{p}} \psi_{1 / \sqrt{p}} h\left(A^{\prime}, g_{t / p^{2}}^{\Omega \cdot\left(X,\left.F_{p}\right|_{X}\right)}\right) & =\pi_{*} c_{t / 4} p^{n}+\mathcal{O}\left(p^{n-1}\right), \\
\left.\psi_{1 / \sqrt{p} \frac{1}{\sqrt{t}} h^{\wedge}\left(A^{\prime}, g_{t / p^{2}} \Omega^{\cdot}\left(X,\left.F_{p}\right|_{X}\right)\right.}\right) & =\pi_{*} \frac{1}{\sqrt{t}} d_{t / 4} p^{n+1}+\mathcal{O}\left(p^{n}\right) .
\end{aligned}
$$

In particular, if $K \subset S$ is compact, there exists $C>0$ such that, for $p \in \mathbf{N}^{*}, 0<t \leqslant 1$,

$$
\left|p^{-n-1} \psi_{1 / \sqrt{p}} h^{\wedge}\left(A^{\prime}, g_{t / p^{2}}^{\Omega^{\cdot}\left(X,\left.F_{p}\right|_{X}\right)}\right)\right| \leqslant C \sqrt{t} \quad \text { on } K \text {. }
$$

If $\widehat{\theta}^{\mathfrak{p}}$ is nondegenerate, given $\alpha>0$ and a compact set $K \subset S$, there exist $c>0, c^{\prime}>$ $0, C>0$ such that, for $t \geqslant \alpha, p \in \mathbf{N}$,

$$
\begin{aligned}
\left|h\left(A^{\prime}, g_{t}^{\Omega \cdot\left(X,\left.F_{p}\right|_{X}\right)}\right)\right| \leqslant C \exp \left(-c\left(p^{2}-c^{\prime}\right) t\right) & \text { on } K, \\
\left|h^{\wedge}\left(A^{\prime}, g_{t}^{\Omega \cdot\left(X,\left.F_{p}\right|_{X}\right)}\right)\right| \leqslant C \exp \left(-c\left(p^{2}-c^{\prime}\right) t\right) & \text { on } K .
\end{aligned}
$$

Proof. To prove our theorem, we may as well make $2 i \pi=1$ in the normalization of the various forms. We may and we will assume $S$ to be compact. First, we establish (6.23). By equation (6.22) in Theorem 6.3, with $L$ replaced by $L^{p}$ and $F$ by $F_{p}$, we get

$$
\begin{aligned}
& h\left(A^{\prime}, g_{t / p^{2}}^{\Omega^{\cdot}\left(X,\left.F_{p}\right|_{X}\right)}\right) \\
& \quad=\int_{X}\left[\operatorname{Tr}_{\mathrm{s}} \Lambda^{\cdot\left(T^{*} X\right)}\left[Q_{t / p^{2}}^{\mathbf{F}}(x, x)\right]^{z} \int_{G_{\mathbf{C}, r, x}} \chi_{p}\left(g^{-1}\right) S_{t / p^{2}, x}^{\mathbf{F}}(d g)\right] d v_{X}(x) \\
& \quad=\int_{X}\left[\int_{P_{G_{\mathbf{C}}, x}} \operatorname{Tr}_{\mathrm{S}} \Lambda^{\cdot\left(T^{*} X\right)}\left[P_{t / p^{2}}^{\mathbf{F}}\left(u, u^{\prime}\right)\right]^{z} \chi_{p}\left(u^{\prime-1} u\right) d q\left(u^{\prime}\right)\right] d v_{X}(x) .
\end{aligned}
$$


For $a \in \mathbf{R}$, the action of $\theta_{a}$ on $\mathbf{R}[z] \widehat{\otimes} \Lambda^{*}\left(T^{*} S\right)$ was defined in $\S 5.9$. Set

$$
L_{p, t}^{\mathbf{F}}=\theta_{1 / \sqrt{p}} L_{t}^{\mathbf{F}} \theta_{\sqrt{p}}
$$

To the operator $\exp \left(-L_{p, t}^{\mathbf{F}}\right)$ we associate kernels and measures which are denoted with an extra subscript $p$. We use the corresponding notation for the operator $L_{t}^{\mathbf{F} \prime}$. By (6.26), we get

$$
\begin{aligned}
& p^{-1 / 2} \psi_{1 / \sqrt{p}} h\left(A^{\prime}, g_{t / p^{2}}^{\Omega \cdot\left(X, F_{p} \mid X\right)}\right) \\
& =\int_{X}\left[\operatorname{Tr}_{\mathrm{s}} \Lambda^{\cdot\left(T^{*} X\right)}\left[Q_{p, t / p^{2}}^{\mathbf{F}}(x, x)\right]^{z} \int_{G_{\mathbf{C}, r, x}} \chi_{p}\left(g^{-1}\right) S_{p, t / p^{2}, x}^{\mathbf{F}}(d g)\right] d v_{X}(x) \\
& =\int_{X}\left[\int_{P_{G_{\mathbf{C}}, x}} \operatorname{Tr}_{\mathrm{s}}{ }^{\Lambda^{\prime}\left(T^{*} X\right)}\left[P_{p, t / p^{2}}^{\mathbf{F}}\left(u, u^{\prime}\right)\right]^{z} \chi_{p}\left(u^{\prime-1} u\right) d q\left(u^{\prime}\right)\right] d v_{X}(x) .
\end{aligned}
$$

Set

$$
\Lambda_{p, t}=\theta_{1 / \sqrt{p}} \Lambda_{4 t / p^{2}} \theta_{\sqrt{p}}
$$

By (6.11), we obtain

$$
\theta_{1 / \sqrt{p}} L_{4 t / p^{2}}^{\mathscr{F}} \theta_{\sqrt{p}}=-\frac{t}{p^{2}}\left({ }^{1} \nabla_{2 t / p, e_{i}}^{\pi^{*} \Lambda^{\cdot}\left(T^{*} S\right) \widehat{\otimes} \Lambda^{\cdot}\left(T^{*} X\right) \otimes \mathscr{F}, u}-\frac{z}{2 \sqrt{t}} \sqrt{p} \widehat{c}\left(e_{i}\right)\right)^{2}+\Lambda_{p, t} .
$$

In what follows, we will systematically underline the elements of $\mathfrak{g}_{r}$ or $U \mathfrak{g}_{r}$. This is because, as in $\S 1$, the elements of $U \mathfrak{g}_{r}$, viewed as differential operators along the fibres of $G_{r}$, will have to be distinguished from corresponding elements in $S \mathfrak{g}_{r}$, viewed as differential operators with constant coefficients along the fibres of $\mathfrak{g}_{r}$. In particular, instead of (5.61), we use the notation

$$
\underline{\mathcal{R}}=\frac{1}{4}\left\langle e_{i}, R^{T X} e_{j}\right) \widehat{c}\left(e_{i}\right) \widehat{c}\left(e_{j}\right)-\underline{\theta}^{\mathfrak{p}, 2} .
$$

By (5.63), (6.29), we get

$$
\begin{aligned}
& \Lambda_{p, t}=\frac{t K^{X}}{4 p^{2}}+\frac{t}{2 p} c\left(e_{i}\right) c\left(e_{j}\right) \frac{\mathcal{\mathcal { R }}}{p}\left(e_{i}, e_{j}\right)+\frac{1}{2} f^{\alpha} f^{\beta} \frac{\underline{\mathcal{R}}}{p}\left(f_{\alpha}^{H}, f_{\beta}^{H}\right) \\
& +\frac{\sqrt{t} c\left(e_{i}\right)}{\sqrt{p}} f^{\alpha} \frac{\underline{\mathcal{R}}}{p}\left(e_{i}, f_{\alpha}^{H}\right)+t \frac{\left|\underline{\widehat{\theta}}^{\mathfrak{p}}\right|^{2}}{p^{2}}+\sqrt{t} f^{\alpha} \frac{\widehat{c}\left(e_{i}\right)}{p^{1 / 2}} \nabla_{f_{\alpha}^{H}}^{T X \otimes \mathfrak{g}_{r}, u} \frac{\widehat{\widehat{\theta}}^{\mathfrak{p}}\left(e_{i}\right)}{p} \\
& +\frac{t}{2 p} \widehat{c}\left(e_{i}\right) \widehat{c}\left(e_{j}\right) \frac{\widehat{\widehat{\theta}}^{\mathfrak{p}, 2}\left(e_{i}, e_{j}\right)}{p}+\frac{t}{p} c\left(e_{i}\right) \widehat{c}\left(e_{j}\right) \nabla_{e_{i}}^{T X \otimes \mathfrak{g}_{r}, u} \frac{\widehat{\widehat{\theta}}^{\mathfrak{p}}}{p}\left(e_{j}\right) \\
& +\frac{\sqrt{t}}{p^{1 / 2}} z c\left(e_{i}\right) \frac{\underline{\theta}^{\mathfrak{p}}}{p}\left(e_{i}\right)+z f^{\alpha} \frac{\underline{\theta}^{\mathfrak{p}}}{p}\left(f_{\alpha}^{H}\right) .
\end{aligned}
$$

Let $x \in M$, and let $X$ be the fibre of $\pi$ containing $x$. Along this fibre, we take a local geodesic coordinate system centred at $x$; i.e., for $\epsilon>0$ small enough, we identify the open ball $B^{T_{x} X}(0, \epsilon) \subset T_{x} X$ with the open ball $B^{X}(x, \epsilon)$ via the map $Y \in T_{x} X \rightarrow$ $\exp _{x}(Y) \in X$. We trivialize fibrewise $\mathbf{R}[z] \widehat{\otimes} \Lambda^{*}\left(T^{*} S\right) \widehat{\otimes} \Lambda^{*}\left(T^{*} X\right)$ near $x$ using parallel 
transport with respect to the fibrewise connection ${ }^{1} \nabla_{2 t / p}^{\Lambda^{\cdot}\left(T^{*} S\right) \widehat{\otimes} \Lambda^{\cdot}\left(T^{*} X\right)}-\frac{z \sqrt{p}}{2 \sqrt{t}} \widehat{c}(\cdot)$ along the geodesics centred at $x$. Also, we trivialize $P_{K}$ fibrewise by parallel transport with respect to the connection $\theta^{\mathfrak{k}}$ along these geodesics, so that

$$
\left.P_{K}\right|_{B^{X}(0, \epsilon)} \simeq B^{T_{X} X}(0, \epsilon) \times K,\left.\quad P_{G}\right|_{B^{X}(0, \epsilon)} \simeq B^{T_{x} X}(0, \epsilon) \times G .
$$

In (6.33), we can as well replace $K, G$ by $U, G_{\mathbf{C}}$. Under the identifications in (6.33),

$$
G_{r} \simeq G, \quad \mathfrak{g}_{r}=\mathfrak{g}, \quad U \mathfrak{g}_{r} \simeq U \mathfrak{g},
$$

and the left action of $G_{r}$ on $P_{G}$ is just the left action of $G$ on $G$. Also, in (6.34), $\mathfrak{g}_{r}$ is identified with the right-invariant vector fields on $G$, and $U \mathfrak{g}_{r}$ acts on $G$ as right-invariant differential operators.

For $\epsilon>0$ small enough, via the exponential map, we identify a neighbourhood of 0 in $\mathfrak{g}$ with a neighbourhood of 1 in $G$. This identification extends to a holomorphic identification of a neighbourhood $B^{\mathfrak{g}_{\mathbf{C}}}(0, \epsilon)$ of 0 in $\mathfrak{g}_{\mathbf{C}}$ with a neighbourhood $B^{G_{\mathbf{C}}}(0, \epsilon)$ of 1 in $G_{\mathbf{C}}$. Let $\mathcal{O}\left(B^{G_{\mathbf{C}}}(0, \epsilon)\right)$ be the vector space of complex holomorphic functions on $B^{\mathfrak{g}_{\mathrm{C}}}(0, \epsilon)$. Under the above identifications, the operator $\theta_{1 / \sqrt{p}} L_{4 t / p^{2}}^{\mathscr{F}} \theta_{\sqrt{p}}$ acts on

$$
C^{\infty}\left(B^{T_{x} X}(0, \epsilon), \mathbf{R}[z] \widehat{\otimes} \Lambda^{\prime}\left(T^{*} S\right) \widehat{\otimes} \Lambda^{\prime}\left(T_{x}^{*} X\right)\right) \otimes_{\mathbf{C}} \mathcal{O}\left(B^{\mathfrak{g}_{\mathbf{C}}}(0, \epsilon)\right) .
$$

Let $Y, A$ denote the tautological sections of $T_{x} X, \mathfrak{g}_{\mathbf{C}}$. For $s>0, a>0$, set

$$
K_{s, a} f(Y, A)=f(s Y, a A) .
$$

Put

$$
M_{p, t}^{\mathscr{F}}=K_{\sqrt{t} / p, 1 / p} \theta_{1 / \sqrt{p}} L_{4 t / p^{2}}^{\mathscr{F}} \theta_{\sqrt{p}} K_{p / \sqrt{t}, p} .
$$

We introduce another copy $\widehat{T_{x} X}$ of $T_{x} X$. If $e \in T_{x} X$, let $\widehat{e}$ denote the corresponding element in $\widehat{T_{x} X}$. Let $e^{*} \in T^{*} X$ correspond to $e$ by the metric of $T_{x} X$, and let $\widehat{e}^{*}$ be the corresponding element in $\widehat{T^{*} X}$.

We make a Getzler rescaling [31] on the Clifford variables $c\left(e_{i}\right), \widehat{c}\left(e_{i}\right)$. For $e \in T_{X} X, s>$ 0 , set

$$
c_{s}(e)=\frac{1}{\sqrt{s}} e^{*} \wedge-\sqrt{s} i_{e}, \quad \widehat{c}_{s}(e)=\frac{1}{\sqrt{s}} \widehat{e}^{*} \wedge+\sqrt{s} i_{\widehat{e}} .
$$

This notation is compatible with that in $\S 1.8$. We denote by $N_{p, t}^{\mathscr{F}}$ the operator obtained from $M_{p, t}^{\mathscr{F}}$ by replacing the $c\left(e_{i}\right), \widehat{c}\left(e_{i}\right)$ by the $c_{t / p}\left(e_{i}\right), \widehat{c}_{1 / p}\left(e_{i}\right)$.

Recall that $\sigma\left(\mathcal{A}_{t}^{2}\right)$ is given by (2.19). As explained in $\S 1.8, \sigma\left(\mathcal{A}_{t}^{2}\right)$ is viewed as a differential operator with constant coefficients acting along the fibre $\mathfrak{g}_{r, x}$.

In what follows, tensors will be evaluated at $x$. Let $\Delta^{T X}$ be the fibrewise Laplacian along $T X$. Set

$$
N_{\infty, t}^{\mathscr{F}}=-\Delta^{T X}+\sigma\left(\mathcal{A}_{t}^{2}\right)+z \theta^{\mathfrak{p}} .
$$

The $e^{i}, f^{\alpha}$ generate $T^{*} M$, and will be considered as differential forms on $M$. We claim that, as $p \rightarrow+\infty$, we have the convergence of differential operators on $T_{x} X$,

$$
N_{p, t}^{\mathscr{F}} \rightarrow N_{\infty, t}^{\mathscr{F}} .
$$


Equation (6.40) says that we have uniform convergence of the coefficients of the operators as well as their derivatives of any order over compact subsets.

To establish (6.40), let us review the various terms in the right-hand side of $(6.30)$. The contribution of the first term in the right-hand side of $(6.30)$ to the limit of $N_{p, t}^{\mathscr{F}}$ is just $-\Delta^{T X}$. Indeed, local index-theoretic techniques, which include the local families index theorem, show that the connection ${ }^{1} \nabla_{2 t / p}^{\Lambda^{*}\left(T^{*} S\right)} \widehat{\otimes} \Lambda^{\cdot}\left(T^{*} X\right)-\frac{z \sqrt{p}}{2 \sqrt{t}} \widehat{c}(\cdot)$ only contributes trivially to the limit. So we concentrate on the contribution of $\theta^{\mathfrak{k}}$. In this trivialization by parallel transport with respect to $\theta^{\mathfrak{k}}$, let $\Gamma^{\mathfrak{k}}$ be the connection form for $\theta^{\mathfrak{k}}$. Since the curvature of $\theta^{\mathfrak{k}}$ is given by $-\theta^{\mathfrak{p}, 2}$, we have

$$
\Gamma_{Y}^{\mathfrak{k}}=-\frac{1}{2} \theta^{\mathfrak{p}, 2}(Y, \cdot)+\mathcal{O}\left(|Y|^{2}\right) .
$$

For each index $i$, inside the square in the right-hand side of (6.30), the asymptotic contribution of $\theta^{\mathfrak{k}}$ is given by

$$
p \frac{t}{2 p^{2}} \mathcal{O}(Y),
$$

the first factor $p$ coming from the rescaling in the variable $A$. The expression in (6.42) tends to 0 as $p \rightarrow+\infty$. The above establishes our claim on the contribution of the first term in (6.30).

We claim that, as $p \rightarrow+\infty$, the contribution of $\Lambda_{p, t}$ to the limit of $N_{p, t}^{\mathscr{F}}$ is given by $\sigma\left(\mathcal{A}_{t}^{2}\right)+z \theta^{\mathfrak{p}}$. This follows from arguments similar to the ones in the proof of Proposition 1.4, from (2.19), and from (6.31), (6.32). This completes the proof of (6.40).

The operators in (6.40) act on functions which are holomorphic on $\mathfrak{g}_{\mathbf{C}}$. Therefore, it is a priori not possible to deduce anything for the corresponding heat operators, because the classical arguments we have in hand necessitates the convergence of operators acting on smooth functions. However, in $\S 6.3$, we defined lifts $L_{t}^{\mathbf{F}}, L_{t}^{\mathbf{F} \prime}$ of $L_{t}^{\mathscr{F}}$. The arguments we gave when proving (6.40) have a counterpart for the operators $L_{t}^{\mathbf{F}}, L_{t}^{\mathbf{F}}$ constructed in $\S 6.3$. From these operators, one can define operators $M_{p, t}^{\mathbf{F}}, M_{p, t}^{\mathbf{F} \prime}$ and $N_{p, t}^{\mathbf{F}}, N_{p, t}^{\mathbf{F} \prime}$ as in (6.37), (6.38).

We claim that the operators $N_{p, t}^{\mathbf{F}}, N_{p, t}^{\mathbf{F} \prime}$ have asymptotics which are similar to the asymptotics of $N_{p, t}^{\mathscr{F}}$ in (6.40); i.e., there are differential operators $N_{\infty, t}^{\mathbf{F}}, N_{\infty, t}^{\mathbf{F} \prime}$ with constant coefficients on $T_{x} X \times \mathfrak{g}_{\mathbf{C}}$ such that, as $p \rightarrow+\infty$,

$$
N_{p, t}^{\mathbf{F}} \rightarrow N_{\infty, t}^{\mathbf{F}}, \quad N_{p, t}^{\mathbf{F} \prime} \rightarrow N_{\infty, t}^{\mathbf{F} \prime} .
$$

This is because the operators $L_{t}^{\mathbf{F}}, L_{t}^{\mathbf{F} \prime}$ have exactly the same kind of homogeneity as the operator $L_{t}^{\mathscr{F}}$ itself. When restricting these operators to functions that are holomorphic along $\mathfrak{g}_{\mathbf{C}}$, the operators $N_{\infty, t}^{\mathbf{F}}, N_{\infty, t}^{\mathbf{F} \prime}$ coincide with $N_{\infty, t}^{\mathscr{F}}$. The operators $N_{\infty, t}^{\mathbf{F}}, N_{\infty, t}^{\mathbf{F} \prime}$ can be written in the form

$$
N_{\infty, t}^{\mathbf{F}}=-\Delta^{T X}+\mathcal{K}^{\mathbf{F}}, \quad N_{\infty, t}^{\mathbf{F} \prime}=-\Delta^{T X}+\mathcal{K}^{\mathbf{F}} .
$$

Comparing with (6.39), we find that $\mathcal{K}^{\mathbf{F}}, \mathcal{K}^{\mathbf{F} \prime}$ are differential operators along the fibres of $P_{G_{\mathbf{C}}}$ that are lifts of $\sigma\left(\mathcal{A}_{t}^{2}\right)+z \theta^{\mathfrak{p}}$, the operator $\mathcal{K}^{\mathbf{F} \prime}$ being fibrewise elliptic.

We will now take the asymptotics as $p \rightarrow \infty$ in the right-hand side of (6.28). 
(1) We claim that, given $x \in X$, the computation of the asymptotics of any of the two integrands at $x$ can be made to be local near $x, 1 \in G_{\mathbf{C}, x}$. In $\S 6.3$, we have equipped $\mathfrak{u}$ with an $\operatorname{Ad}(U)$-invariant scalar product, $\mathfrak{g}_{\mathbf{C}}=\mathfrak{u} \oplus i \mathfrak{u}$ with the corresponding scalar product, and $G_{\mathbf{C}}$ with the associated right-invariant metric. Let $d^{G_{\mathbf{C}}}$ be the distance on $G_{\mathbf{C}}$. From the metrics of $X$ and $G_{\mathbf{C}}$, and from the connection $\theta^{\mathfrak{k}}$, we obtain a Riemannian metric $g^{T \mathcal{P}_{G}}$ on $\mathcal{P}_{G_{\mathbf{C}}}$, which is invariant under the right action of $G_{\mathbf{C}}$ on $\mathcal{P}_{G_{\mathbf{C}}}$. Let $d^{\mathcal{P}_{G_{\mathbf{C}}}}$ be the associated distance.

The fibre $\mathcal{P}_{G_{\mathbf{C}}}$ is complete for this metric. Using finite propagation speed, we find that, given $\alpha>0, M>0$, for $d^{\mathcal{P}_{G_{\mathbf{C}}}}\left(u, u^{\prime}\right) \geqslant \alpha, 0<t \leqslant M$,

$$
\left|P_{t}^{\mathbf{F}^{\prime}}\left(u, u^{\prime}\right)\right| \leqslant c \exp \left(-C d^{\mathcal{P}_{G_{\mathbf{C}}}, 2}\left(u, u^{\prime}\right) / t\right) .
$$

Recall that $F_{p}=H^{(0,0)}\left(N, L^{p}\right)$ is equipped with a $U$-invariant Hermitian metric. If $A \in \operatorname{End}\left(F_{p}\right)$, we denote by $\|A\|_{p}$ the norm of $A$ with respect to this Hermitian product. By equation (3.17) in Theorem 3.1, and by proceeding as in (3.42), there exists $C^{\prime}>0$ such that, if $g \in G_{\mathbf{C}}, p \in \mathbf{N}$,

$$
\left\|\rho_{p} g\right\|_{p} \leqslant \exp \left(C^{\prime} p d^{G_{\mathbf{C}}}(1, g)\right), \quad\left\|\rho_{p} g^{-1}\right\|_{p} \leqslant \exp \left(C^{\prime} p d^{G_{\mathbf{C}}}(1, g)\right) .
$$

Moreover, we have the trivial inequality

$$
\left|\chi_{p}(g)\right| \leqslant\left\|\rho_{p} g\right\|_{p} \operatorname{dim} F_{p} .
$$

By (3.41), (6.46), and (6.47), there exist $c^{\prime}>0, C^{\prime}>0$ such that, for $g \in G_{\mathbf{C}}, p \in \mathbf{N}$,

$$
\begin{aligned}
& \left|\chi_{p}(g)\right| \leqslant c^{\prime} p^{n} \exp \left(C^{\prime} p d^{G_{\mathbf{C}}}(1, g)\right), \\
& \left|\chi_{p}\left(g^{-1}\right)\right| \leqslant c^{\prime} p^{n} \exp \left(C^{\prime} p d^{G_{\mathbf{C}}}(1, g)\right) .
\end{aligned}
$$

Take $x \in X, u \in P_{K, x}, u^{\prime} \in P_{G_{\mathbf{C}}, x}$, so that $u^{\prime}=u g, g \in G_{\mathbf{C}}$. Clearly,

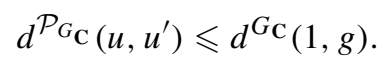

Let $s \in[0,1] \rightarrow u_{s} \in \mathcal{P}_{G_{\mathbf{C}}}$ be a geodesic in $\mathcal{P}_{G_{\mathbf{C}}}$ that connects $u$ and $u^{\prime}$. Let $s \in[0,1] \rightarrow u_{s}^{H} \in \mathcal{P}_{K}$ be the corresponding horizontal curve with respect to the connection $\theta^{\mathfrak{k}}$ such that $u_{0}^{H}=u$. Let $s \in[0,1] \rightarrow g_{s} \in G_{\mathbf{C}}$ be such that $u_{s}=u_{s}^{H} g_{s}$, and let $\ell$ be the length of this curve in $G_{\mathbf{C}}$. Then

$$
d^{G_{\mathbf{C}}}\left(1, g_{1}\right) \leqslant \ell \leqslant d^{\mathcal{P}_{G_{\mathbf{C}}}}\left(u, u^{\prime}\right)
$$

Clearly, there is $k \in K$ such that $u_{1}^{H}=u k$, and so

$$
k g_{1}=g \text {. }
$$

Since the group $K$ is compact, by (6.51), there is $C>0$ such that

$$
d^{G_{\mathbf{C}}}(1, g) \leqslant d^{G_{\mathbf{C}}}\left(1, g_{1}\right)+C .
$$

By (6.50), (6.52), we conclude that

$$
d^{G_{\mathbf{C}}}(1, g) \leqslant d^{\mathcal{P}_{G_{\mathbf{C}}}}\left(u, u^{\prime}\right)+C .
$$


By $(6.45),(6.48)$, and (6.53), for $d^{\mathcal{P}_{G} \mathbf{C}}\left(u, u^{\prime}\right) \geqslant \alpha, 0<t \leqslant M, p \in \mathbf{N}^{*}$,

$$
\left|P_{t / p^{2}}^{\mathbf{F}}\left(u, u^{\prime}\right) \chi_{p}\left(u^{\prime-1} u\right)\right| \leqslant c \exp \left(-C^{\prime} p^{2} d^{\mathcal{P}_{G_{C}}, 2}\left(u, u^{\prime}\right)\right) .
$$

By (6.54), we deduce that, given $t>0$, as $p \rightarrow+\infty$,

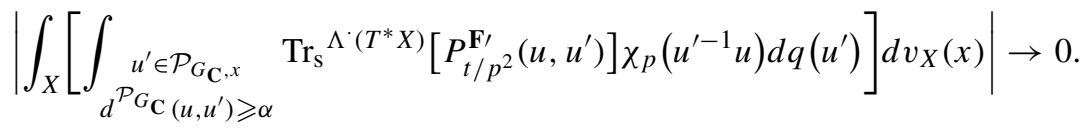

(2) We use the trivializations indicated after (6.32). The above arguments also indicate that, to compute the asymptotics of $(6.28)$ as $p \rightarrow+\infty$, near $(x, 1) \in X \times G_{\mathbf{C}}$, we can replace $X \times G_{\mathbf{C}}$ by $T_{x} X \times \mathfrak{g}_{\mathbf{C}}$, and replace the operator $L_{t}^{\mathbf{F} \prime}$ by an operator $\underline{L}_{t}^{\mathbf{F}}$ on $T_{X} X \times \mathfrak{g}_{\mathbf{C}}$ that is trivial outside a small ball centred at 0 in $T_{X} X$. We define the operators $\underline{M}_{p, t}^{\mathbf{F} \prime}, \underline{N}_{p, t}^{\mathbf{F} \prime}$ from $\underline{L}_{t}^{\mathbf{F} \prime}$ exactly as before.

Up to permutation, the monomial $\prod_{i=1}^{m} c\left(e_{i}\right) \widehat{c}\left(e_{i}\right)$ is the only one whose supertrace is nonzero, and, moreover,

$$
\operatorname{Tr}_{\mathrm{s}} \Lambda^{\cdot\left(T^{*} X\right)}\left[\prod_{i=1}^{m} c\left(e_{i}\right) \widehat{c}\left(e_{i}\right)\right]=(-2)^{m}
$$

Let $\widehat{\operatorname{Tr}}_{\mathrm{S}}$ be the functional on the algebra of operators generated by the $e_{i}, i_{e_{i}}, \widehat{e}^{i}, i_{\widehat{e}_{i}}, 1 \leqslant i \leqslant m$ that vanishes on all monomials except, up to permutation, on $\prod_{i=1}^{m} e^{i} \widehat{e}^{i}$ and is such that

$$
\widehat{\operatorname{Tr}_{\mathrm{S}}}\left[\prod_{i=1}^{m} e^{i} \widehat{e}^{i}\right]=(-1)^{m}
$$

Let $\underline{P}_{p, t}^{\mathbf{F} \prime}\left(Y,\left(Y^{\prime}, A^{\prime}\right)\right)$ be the smooth kernel associated with the operator $\exp \left(-\underline{N}_{p, t}^{\mathbf{F}}\right)$ with respect to the volume $d Y^{\prime} d A^{\prime}$. Take $\alpha>0$ small enough. To compute the asymptotics of (6.28) as $p \rightarrow+\infty$, we should compute at each $x \in X$ the asymptotics as $p \rightarrow+\infty$ of

$$
2^{m} \int_{\substack{A \in \mathfrak{g}_{\mathrm{C}} \\|A| \leqslant \alpha p}} \widehat{\operatorname{Tr}}_{\mathrm{s}}\left[\underline{P}_{p, t}^{\mathbf{F} /}(0,(0, A))\right]\left(\delta_{1 / p} \chi_{p}\right)(-A) d A .
$$

Let $\underline{P}_{\infty, t}^{\mathbf{F} \prime}(0,(Y, A))$ be the smooth kernel associated with the operator $\exp \left(-\underline{N}_{\infty, t}^{\mathbf{F} \prime}\right)$. By $(6.43)$, it is easy to deduce that, as $p \rightarrow+\infty$,

$$
\underline{P}_{p, t}^{\mathbf{F} \prime}(0,(Y, A)) \rightarrow \underline{P}_{\infty, t}^{\mathbf{F} \prime}(0,(Y, A)) .
$$

Combining equation (3.35) in Theorem 3.6, (6.59), and dominated convergence, as $p \rightarrow+\infty$

$$
\begin{gathered}
2^{m} p^{-n} \int_{\substack{A \in \mathfrak{g}_{\mathbf{C}} \\
|A| \leqslant \alpha p}} \widehat{\operatorname{Tr}}_{\mathrm{s}}\left[\underline{P}_{p, t}^{\mathbf{F} \prime}(0,(0, A))\left(\delta_{1 / p} \chi_{p}\right)(-A)\right] d A \\
\rightarrow 2^{m} \int_{A \in \mathfrak{g}_{\mathbf{C}}} \widehat{\widehat{T r}_{\mathrm{s}}}\left[\underline{P}_{\infty, t}^{\mathbf{F}}(0,(0, A))\right] R(-A) d A .
\end{gathered}
$$


Using (6.28), (6.55), (6.58), and (6.60), as $p \rightarrow+\infty$,

$$
\begin{aligned}
p^{-n} p^{-1 / 2} \psi_{1 / \sqrt{p}} h\left(A^{\prime}, g_{4 t / p^{2}}^{\Omega^{\prime}\left(X,\left.F_{p}\right|_{X}\right)}\right) \\
\rightarrow 2^{m} \int_{X}\left[\int_{A \in \mathfrak{g}_{\mathbf{C}}} \widehat{\operatorname{Tr}}_{\mathrm{s}}\left[\underline{P}_{\infty, t}^{\mathbf{F} \prime}(0,(0, A))\right]^{z} R(-A) d A\right] d v_{X}
\end{aligned}
$$

(3) Recall that $\mathcal{K}^{\mathbf{F} /}$ was defined in (6.44). As we saw after (6.44), the elliptic operator $\mathcal{K}^{\mathbf{F} \prime}$ is a lift of $\sigma\left(\mathcal{A}_{t}^{2}\right)+z \theta^{\mathfrak{p}}$. Since the function $R(A)$ is holomorphic in $A$, we have the identity,

$$
\int_{A \in \mathfrak{g}_{\mathbf{C}}} \widehat{\operatorname{Tr}_{\mathrm{s}}}\left[\underline{P}_{\infty, t}^{\mathbf{F} \prime}(0,(0, A)) R(-A)\right] d A=(4 \pi)^{-m / 2} \widehat{\operatorname{Tr}}_{\mathrm{s}}\left[\exp \left(-\sigma\left(\mathcal{A}_{t}^{2}\right)-z \theta^{\mathfrak{p}}\right) R(0)\right]
$$

Observe here that, in spite of the fact that $R(-A)$ appears in $(6.61)$, we have not made a corresponding change of signs in $\sigma\left(\mathcal{A}_{t}^{2}\right)+z \theta^{\mathfrak{p}}$. This is because, as we saw in $\S 1.6, A \in \mathfrak{g}_{\mathbf{C}, r}$ acts like the vector field $-A^{N}$, which accounts for (6.62).

By combining (1.28), (2.55), (6.57), (6.61), and (6.62), for $t>0$, we get

$$
p^{-n} \frac{1}{\sqrt{p}} \psi_{1 / \sqrt{p}} h\left(A^{\prime}, g_{4 t / p^{2}}^{\Omega \cdot\left(X,\left.F_{p}\right|_{X}\right)}\right) \rightarrow \pi_{*} c_{t} .
$$

We will now refine (6.63) to obtain the more precise asymptotic expansion in the first identity in (6.23). The fact that, for a given $t>0$, there is an asymptotic expansion as in (6.23) relies on standard arguments based on the corresponding asymptotic expansion of the operators in (6.40). Also, the proof of equation (5.30) in Theorem 5.11 that is given in [18, Theorem 3.16] uses two kinds of argument: an argument of localization of the asymptotics near the diagonal in $X \times X$, and a rescaling of the Clifford variables $c(e) \rightarrow c_{t}(e)$. In the present context, where $t$ is replaced by $t / p^{2}$, the $c(e)$ should be replaced by $c_{t / p^{2}}(e)$. Here, we replace $c(e)$ by the less singular $c_{t / p}(e)$, but we introduce an extra singularity when replacing $\widehat{c}(e)$ by $\widehat{c}_{1 / p}(e)$. Still, the above arguments show easily that we have uniformity in the convergence in (6.23) when $t \rightarrow 0$, so that (6.23) holds uniformly for $0<t \leqslant M$. By (2.68), (5.44), the second equation in (6.23) follows from the first equation. Equation (6.24) follows from (6.23).

If $\widehat{\theta}^{\mathfrak{p}}$ is nondegenerate, by equation (4.19) in Theorem 4.4, by (5.63), and by equation (5.67) in Theorem 5.22, using Kato's comparison principle, for $p \in \mathbf{N}$ and $t \geqslant \alpha>0$,

$$
\begin{aligned}
& \left|h\left(A^{\prime}, g_{t}^{\Omega \cdot\left(X,\left.F_{p}\right|_{X}\right)}\right)\right| \leqslant C \exp \left(-a\left(p^{2}-c^{\prime}\right) t\right) \operatorname{dim}\left(F_{p}\right), \\
& \left|h^{\wedge}\left(A^{\prime}, g_{t}^{\Omega \cdot\left(X,\left.F_{p}\right|_{X}\right)}\right)\right| \leqslant C \exp \left(-a\left(p^{2}-c^{\prime}\right) t\right) \operatorname{dim}\left(F_{p}\right) .
\end{aligned}
$$

By (3.41), (6.64), we get (6.25). The proof of our theorem is completed. 
Remark 6.6. In our proof of Theorem 6.5, we used the second equation in (6.22) instead of the first one. However, the first equation would have done as well. Its apparent drawback is that the operator $L_{t}^{\mathbf{F}}$ is not elliptic in the directions of the fibres of $P_{G_{\mathbf{C}}}$. However, the fact that there is an integral in $g \in G_{\mathbf{C}, r}$ in the right-hand side of (6.22) makes this lack of ellipticity irrelevant. Equation (6.58) should be replaced by a weak convergence of measures. Details are left to the reader.

Also, the reader will have noticed that, when taking the proper limit as $p \rightarrow+\infty$ of equation (5.26), we would just recover equation (2.41), which gives the proper perspective to Theorem 2.5.

\subsection{Convergence results on a Galois cover of $X$}

We assume that $S$ is reduced to a point. Let $\Gamma$ be a discrete group acting freely and properly discontinuously on a manifold $\widehat{X}$, so that $X=\Gamma \backslash \widehat{X}$. Let $\widehat{\pi}: \widehat{X} \rightarrow X$ be the obvious projection. The metric $g^{T X}$ lifts to a $\Gamma$-invariant metric $g^{T \widehat{X}}$ on $T \widehat{X}$.

Let $H$ be a Hermitian vector bundle on $X$. Let $\mathcal{Q}$ be the vector space of continuous kernels $Q\left(z, z^{\prime}\right)$ acting on $C^{b}\left(\widehat{X}, \widehat{\pi}^{*} H\right)$ that commute with $\Gamma$, and such that there exist $c>0, C>0$ such that

$$
\left|Q\left(z, z^{\prime}\right)\right| \leqslant C \exp \left(-c d^{2}\left(z, z^{\prime}\right)\right) \text {. }
$$

One verifies easily that $\mathcal{Q}$ is an algebra. Also, $\operatorname{Tr}[Q(z, z)]$ is a $\Gamma$-invariant function, so that it descends to a continuous function on $X$. Set

$$
\operatorname{Tr}^{\Gamma}[Q]=\int_{X} \operatorname{Tr}[Q(x, x)] d v_{X}
$$

One then verifies easily that $\operatorname{Tr}^{\Gamma}$ is a trace on $\mathcal{Q}$; i.e., it vanishes on commutators. Using finite propagation speed for the wave equation, one can derive the well-known fact that heat kernels on $\widehat{X}$ lie in the algebra $\mathcal{Q}$.

We will now apply the formalism of the previous sections, still assuming $S$ to be a point. For $t>0$, we define $h^{\wedge, \Gamma}\left(A^{\prime}, g_{t}^{\Omega \cdot\left(\widehat{X},\left.\widehat{\pi}^{*} F\right|_{X}\right)}\right) \in \mathbf{R}$ by the same formula as in (5.35), by replacing the supertrace in the right-hand side of (5.35) by the corresponding $\Gamma$-supertrace $\operatorname{Tr}_{\mathrm{s}} \Gamma$.

We claim that the results of Theorem 6.5 are valid for $h^{\wedge, \Gamma}\left(A^{\prime}, g_{t}^{\Omega \cdot\left(\widehat{X}, \widehat{\pi}^{*} F_{p} \mid \widehat{X}\right)}\right)$. The key point is that, as observed in Remark 4.5, if $\widehat{\theta}^{\mathfrak{p}}$ is nondegenerate, the spectral estimate (4.19) is still valid over $\widehat{X}$. Therefore, if $\widehat{\theta}^{\mathfrak{p}}$ is nondegenerate, for $p \in \mathbf{N}$ large enough, we can still define the $\Gamma$-torsion $\mathcal{T}_{h}^{\Gamma}\left(g^{T X}, F_{p}, g^{F_{p}}\right) \in \mathbf{R}$ by a formula similar to (5.45).

Taking into account the fact that $S$ is reduced to a point, we claim that the obvious analogue of Theorem 6.5 holds for $h^{\wedge, \Gamma}\left(A^{\prime}, g_{t}^{\Omega\left(\widehat{X}, \widehat{\pi}^{*} F_{p} \mid \widehat{X}\right)}\right)$. In particular, if $\pi_{*} d_{t} \in \mathbf{R}$ still refers to the object considered in $\S 2$ that is associated with $X$, as $p \rightarrow+\infty$,

$$
h^{\wedge, \Gamma}\left(A^{\prime}, g_{t / p^{2}}^{\Omega \cdot\left(\widehat{\pi},\left.\widehat{\pi}^{*} F_{p}\right|_{\widehat{X}}\right)}\right)=p^{n+1} \pi_{*} d_{t / 4}+\mathcal{O}\left(p^{n}\right) .
$$

Also, the estimates in (6.23)-(6.25) still hold. The proof of the above is strictly similar to the proof of Theorem 6.5.

Given a nontrivial conjugacy class $[\gamma]$ in $\Gamma$, one can instead define an associated $h^{\wedge, \Gamma,[\gamma]}\left(A^{\prime}, g_{t / p^{2}}^{\Omega^{2}\left(\widehat{X}, \widehat{\pi}^{*} F_{p} \mid \widehat{X}\right)}\right)$. One verifies easily that, given $t>0$, there exist $C>0, c>0$ 
such that, as $p \rightarrow+\infty$,

$$
\left|h^{\wedge, \Gamma,[\gamma]}\left(A^{\prime}, g_{t / p^{2}}^{\Omega \cdot\left(\widehat{X},\left.\widehat{\pi}^{*} F_{p}\right|_{\widehat{X}}\right)}\right)\right| \leqslant C \exp \left(-c p^{2}\right),
$$

and, moreover, that the analogues of (6.23)-(6.25) still hold.

Some of the above results will be reobtained in $\S 8$ in the context of locally symmetric spaces.

\section{The asymptotics of the analytic torsion forms}

In this section, when $\widehat{\theta}^{\mathfrak{p}}$ is nondegenerate, we obtain the asymptotics as $p \rightarrow+\infty$ of the analytic torsion forms $\mathcal{T}_{h}\left(T^{H} M, g^{T X}, \nabla^{F_{p}}, g^{F_{p}}\right)$ in terms of the form $W$ that was defined in $\S 2$.

This section is organized as follows. In $\S 7.1$, we state our main result, the proof of which is divided into two key steps, involving large and small values of the parameter $t>0$.

In $\S 7.2$, we obtain the estimate involving large values of $t$.

In $\S 7.3$, we prove the estimate involving small values of $t$.

In $\S 7.4$, we verify the compatibility of our asymptotic formula to known results on the forms $W$ and $\mathcal{T}_{h}\left(T^{H} M, g^{T X}, \nabla^{F_{p}}, g^{F_{p}}\right)$.

In $\S 7.5$, we derive rigidity results on the class of forms $\pi_{*} W$ that are consequences of our asymptotic formula.

Finally, in $\S 7.6$, we obtain the asymptotics of the $\Gamma$-torsion of a Galois covering $\widehat{X}$ of $X$.

\subsection{The main result}

We make the same assumptions as in $\S 6$. Also, we assume $\widehat{\theta}^{\mathfrak{p}}$ to be nondegenerate in the sense of Definition 1.13. Recall that the form $W$ was defined in Definition 2.11.

Theorem 7.1. As $p \rightarrow+\infty$,

$$
p^{-n-1} \psi_{1 / \sqrt{p}} \mathcal{T}_{h}\left(T^{H} M, g^{T X}, \nabla^{F_{p}}, g^{F_{p}}\right)=\pi_{*} W+\mathcal{O}\left(p^{-1}\right)
$$

Proof. For simplicity, we assume $S$ to be compact. By Theorem 4.4, for $p$ large enough, the complexes $\left(\Omega \cdot\left(X,\left.F_{p}\right|_{X}\right), d^{X}\right)$ are exact. By (5.45), for $p$ large enough,

$$
\mathcal{T}_{h}\left(T^{H} M, g^{T X}, \nabla^{F_{p}}, g^{F_{p}}\right)=-\int_{0}^{+\infty} h^{\wedge}\left(A^{\prime}, g_{t}^{\Omega \cdot\left(X, F_{p} \mid X\right)}\right) \frac{d t}{t} .
$$

We rewrite (7.2) in the form

$$
\begin{aligned}
\mathcal{T}_{h}\left(T^{H} M, g^{T X}, \nabla^{F_{p}}, g^{F_{p}}\right)= & -\int_{0}^{p} h^{\wedge}\left(A^{\prime}, g_{t / p^{2}}^{\Omega^{\cdot}\left(X,\left.F_{p}\right|_{X}\right)}\right) \frac{d t}{t} \\
& -\int_{1 / p}^{+\infty} h^{\wedge}\left(A^{\prime}, g_{t}^{\Omega \cdot\left(X,\left.F_{p}\right|_{X}\right)}\right) \frac{d t}{t} .
\end{aligned}
$$


In the remainder of the section, we will show that there exists $c>0$ such that, as $p \rightarrow+\infty$,

$$
\begin{aligned}
& -\int_{1 / p}^{+\infty} h^{\wedge}\left(A^{\prime}, g_{t}^{\Omega \cdot\left(X, F_{p} \mid X\right)}\right) \frac{d t}{t}=\mathcal{O}\left(e^{-c p}\right), \\
& -p^{-n-1} \psi_{1 / \sqrt{p}} \int_{0}^{p} h^{\wedge}\left(A^{\prime}, g_{t / p^{2}}^{\Omega \cdot\left(X, F_{p} \mid X\right)}\right) \frac{d t}{t}=\pi_{*} W+\mathcal{O}\left(p^{-1}\right),
\end{aligned}
$$

from which (7.1) follows.

\subsection{A proof of the first equation in (7.4)}

As in $\S 4.3$, we denote by $D_{p}^{X}$ the operator $D^{X}$ acting on $\Omega\left(X,\left.F_{p}\right|_{X}\right)$. Let $\lambda_{p} \geqslant 0$ be the lowest eigenvalue of $D_{p}^{X, 2}$. Take $\alpha>0$. For $t \geqslant \alpha$, we get

$$
\operatorname{Tr}\left[\exp \left(-t D_{p}^{X, 2}\right)\right] \leqslant \exp \left(-\lambda_{p}(t-\alpha)\right) \operatorname{Tr}\left[\exp \left(-\alpha D_{p}^{X, 2}\right)\right]
$$

By equation (4.19) in Theorem 4.4, there exist $c>0, C>0$ such that

$$
\lambda_{p} \geqslant c p^{2}-C .
$$

Taking $\alpha=1 / 4 p$ in (7.5), and using (7.6), for $p \in \mathbf{N}$ large enough, and for $t \geqslant 1 / p$, we get

$$
\operatorname{Tr}\left[\exp \left(-t D_{p}^{X, 2}\right)\right] \leqslant C \exp (-c(p+t)) \operatorname{Tr}\left[\exp \left(-D_{p}^{X, 2} / 4 p\right)\right] .
$$

By (4.13), (4.18), and using Kato's comparison principle, we get

$$
\operatorname{Tr}\left[\exp \left(-D_{p}^{X, 2} / 4 p\right)\right] \leqslant C p^{m / 2}\left(\operatorname{dim} F_{p}\right) \exp (-c p) .
$$

By (3.41), (7.8), we obtain

$$
\operatorname{Tr}\left[\exp \left(-D_{p}^{X, 2} / 4 p\right)\right] \leqslant C p^{m / 2+n} \exp (-c p) .
$$

By (7.7), (7.9), there exist $C>0, c>0$ such that, for $p \in \mathbf{N}$ large enough, and for $t \geqslant 1 / p$,

$$
\operatorname{Tr}\left[\exp \left(-t D_{p}^{X, 2}\right)\right] \leqslant C \exp (-c(p+t)) .
$$

By (5.35), there exist $C>0, k \in \mathbf{N}$ such that

$$
\left|h^{\wedge}\left(A^{\prime}, g_{t}^{\Omega^{\prime}\left(X,\left.F_{p}\right|_{X}\right)}\right)\right| \leqslant C\left(1+t^{-k}\right) \operatorname{Tr}\left[\exp \left(-t D_{p}^{X, 2} / 8\right)\right] .
$$

By (7.10), (7.11), there exist $C>0, c>0$ such that, for $p \in \mathbf{N}$ large enough, and for $t \geqslant 1 / p$,

$$
\left|h^{\wedge}\left(A^{\prime}, g_{t}^{\Omega \cdot\left(X, F_{p} \mid X\right)}\right)\right| \leqslant C \exp (-c p-c t) .
$$

By (7.12), we get the first equation in (7.4). 


\subsection{A proof of the second equation in (7.4)}

Note that

$$
\begin{aligned}
\int_{0}^{p} h^{\wedge}\left(A^{\prime}, g_{t / p^{2}}^{\Omega \cdot\left(X,\left.F_{p}\right|_{X}\right)}\right) \frac{d t}{t}= & \int_{0}^{1} h^{\wedge}\left(A^{\prime}, g_{t / p^{2}}^{\Omega \cdot\left(X,\left.F_{p}\right|_{X}\right)}\right) \frac{d t}{t} \\
& +\int_{1}^{p} h^{\wedge}\left(A^{\prime}, g_{t / p^{2}} \cdot\left(X, F_{p} \mid X\right)\right) \frac{d t}{t}
\end{aligned}
$$

By equation (6.23) in Theorem 6.5, as $p \rightarrow+\infty$,

$$
p^{-n-1} \psi_{1 / \sqrt{p}} \int_{0}^{1} h^{\wedge}\left(A^{\prime}, g_{t / p^{2}}^{\Omega \cdot\left(X,\left.F_{p}\right|_{X}\right)}\right) \frac{d t}{t}=\int_{0}^{1} \pi_{*} d_{t / 4} \frac{d t}{t}+\mathcal{O}\left(p^{-1}\right) .
$$

To handle the second term in the right-hand side of (7.13), we still use (6.23), combined with the fact to be proved that there exist $c>0, C>0$ such that, for $p \in \mathbf{N}$ large enough, and $1 \leqslant t \leqslant p$,

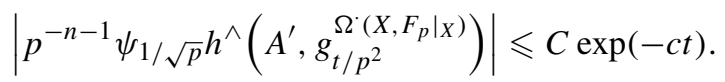

It follows that, as $p \rightarrow+\infty$,

$$
p^{-n-1} \psi_{1 / \sqrt{p}} \int_{1}^{p} h^{\wedge}\left(A^{\prime}, g_{t / p^{2}}^{\Omega^{\cdot}\left(X, F_{p} \mid X\right)}\right) \frac{d t}{t} \rightarrow \int_{1}^{+\infty} \pi_{*} d_{t / 4} \frac{d t}{t} .
$$

By (2.75), (7.13), (7.14), and (7.16), we find that, as $p \rightarrow+\infty$,

$$
-p^{-n-1} \psi_{1 / \sqrt{p}} \int_{0}^{p} h^{\wedge}\left(A^{\prime}, g_{t / p^{2}}^{\Omega^{2}\left(X,\left.F_{p}\right|_{X}\right)}\right) \frac{d t}{t} \rightarrow \pi_{*} W,
$$

which is part of the second equation in (7.4).

So we concentrate on the proof of (7.15). By proceeding as in (5.44) and in the proof of Theorem 6.5, it is enough to show that, for $p \in \mathbf{N}$ large enough, for $1 \leqslant t \leqslant p$,

$$
\left|\frac{1}{\sqrt{p}} p^{-n} \psi_{1 / \sqrt{p}} h\left(A^{\prime}, g_{t / p^{2}}^{\Omega^{\cdot}\left(X,\left.F_{p}\right|_{X}\right)}\right)\right| \leqslant C \exp (-c t) .
$$

Here, we will use equation (5.67). Set

$$
L^{F_{p}}=L_{1}^{F_{p}}
$$

By (5.23), (5.62), and (5.66), we get

$$
L_{t}^{F_{p}}=\theta_{1 / \sqrt{t}} t L^{F_{p}} \theta_{\sqrt{t}} .
$$

By (4.18), there exists $a>0$ such that, for $p \in \mathbf{N}$ large enough,

$$
\rho_{p} \Theta \geqslant 2 a p^{2} \text {. }
$$

Set

$$
L_{t}^{F_{p^{\prime}}}=L_{t}^{F_{p}}-\frac{a p^{2} t}{4}, \quad L^{F_{p^{\prime}}}=L^{F_{p}}-\frac{a p^{2}}{4}
$$


Let $\epsilon \in] 0,1]$ be a lower bound for half of the injectivity radius of the fibres $X$. Let $\psi(u): \mathbf{R}_{+} \rightarrow[0,1]$ be a smooth decreasing function that is equal to 1 for $|u| \leqslant \epsilon / 2$ and to 0 for $|u| \geqslant \epsilon$. Set

$$
\begin{aligned}
F_{t}(x) & =\int_{-\infty}^{+\infty} \cos (u x) \psi(\sqrt{t}|u|) \exp \left(-u^{2} / 4\right) \frac{d u}{\sqrt{4 \pi}} \\
G_{t}(x) & =\int_{-\infty}^{+\infty} \cos (u x)(1-\psi(\sqrt{t}|u|)) \exp \left(-u^{2} / 4\right) \frac{d u}{\sqrt{4 \pi}} .
\end{aligned}
$$

Then

$$
\exp \left(-x^{2}\right)=F_{t}(x)+G_{t}(x) .
$$

Note that $F_{t}(x), G_{t}(x)$ are even holomorphic functions. Therefore there exist holomorphic functions $\widetilde{F}_{t}(x), \widetilde{G}_{t}(x)$ such that

$$
F_{t}(x)=\widetilde{F}_{t}\left(x^{2}\right), \quad G_{t}(x)=\widetilde{G}_{t}\left(x^{2}\right) .
$$

By $(7.24),(7.25)$, we get

$$
\exp (-x)=\widetilde{F}_{t}(x)+\widetilde{G}_{t}(x)
$$

The restrictions of $F_{t}, G_{t}$ to $\mathbf{R}$ lie in the Schwartz space $\mathcal{S}(\mathbf{R})$. Therefore the same is true for the restrictions of $\widetilde{F}_{t}, \widetilde{G}_{t}$ to $\mathbf{R}$.

By (7.22), (7.26), we get

$$
\exp \left(-L_{t}^{F_{p}}\right)=e^{-a p^{2} t / 4}\left(\widetilde{F}_{t}\left(L_{t}^{F_{p^{\prime}}}\right)+\widetilde{G}_{t}\left(L_{t}^{F_{p^{\prime}}}\right)\right) .
$$

Proposition 7.2. There exist $C>0, c>0$ such that, for $p \in \mathbf{N}, 0<t \leqslant 1$,

$$
\left|p^{-n} \operatorname{Tr}_{\mathrm{s}}\left[\widetilde{G}_{t}\left(L_{t}^{F_{p^{\prime}}}\right)\right]\right| \leqslant C \exp (-c / t) .
$$

Proof. Set

$$
H_{t}(x)=\int_{-\infty}^{+\infty} \cos (u x)(1-\psi(|u|)) \exp \left(-u^{2} / 4 t\right) \frac{d u}{\sqrt{4 \pi t}}
$$

Then

$$
G_{t}(x)=H_{t}(x / \sqrt{t}) .
$$

By proceeding as in $[17$, equation (13.23)], given $d>0, k \in \mathbf{N}$, there exist $c>0, C>0$ such that

$$
\sup _{\substack{x \in \mathbf{C} \\|\operatorname{Im}(x)| \leqslant d}}|x|^{k}\left|H_{t}(x)\right| \leqslant C \exp (-c / t) .
$$

Finally, there exists a holomorphic function $\widetilde{H}_{t}(x)$ such that

$$
H_{t}(x)=\widetilde{H}_{t}\left(x^{2}\right) .
$$

By (7.25), (7.30), and (7.32), we get

$$
\widetilde{G}_{t}(x)=\widetilde{H}_{t}(x / t) .
$$


By (7.20), (7.22), and (7.33), we obtain

$$
\widetilde{G}_{t}\left(L_{t}^{F_{p^{\prime}}}\right)=\theta_{1 / \sqrt{t}} \widetilde{H}_{t}\left(L^{F_{p^{\prime}}}\right) \theta_{\sqrt{t}} .
$$

By proceeding as in [9, Proof of Theorem 11.3], one can show that there exist $c>$ $0, C>0$ such that, for $p \in \mathbf{N}, t \in] 0,1]$,

$$
\left|\operatorname{Tr}_{\mathrm{s}}\left[\widetilde{H}_{t}\left(L^{F_{p^{\prime}}}\right)\right]\right| \leqslant C p^{n} \exp (-c / t)
$$

To prove (7.35), let us first assume that $S$ is reduced to a point, so that the part of $L^{F_{p}}$ not containing $z$ is self-adjoint. By (7.21), for $p \in \mathbf{N}$ large enough, we get

$$
\rho_{p} \Theta-a p^{2} \geqslant a p^{2} \text {. }
$$

This estimate is strong enough to control the piece of $L^{F_{p^{\prime}}}$ containing $z$. Let $\Delta^{X}$ be the Laplace-Beltrami operator. By Kato's comparison principle, for $p \in \mathbf{N}$ large enough, we get

$$
\left|\operatorname{Tr}_{\mathrm{s}}\left[\tilde{H}_{t}\left(L^{F_{p^{\prime}}}\right)\right]\right| \leqslant C\left(\operatorname{dim} F_{p}\right) \operatorname{Tr}\left[\tilde{H}_{t}\left(-\Delta^{X} / 4\right)\right] .
$$

By $(7.31)$, for $0<t \leqslant 1$,

$$
\operatorname{Tr}\left[\widetilde{H}_{t}\left(-\Delta^{X} / 4\right)\right] \leqslant c \exp (-C / t) .
$$

By (3.41), (7.38), we get (7.35) when $S$ is reduced to a point. In the general case, Kato's comparison principle is no longer available because $L^{F_{p}}$ is not self-adjoint. However, uniform estimates for the resolvent of $L_{t}^{F_{p}}$ can be obtained that are similar to the ones in [9, Chapter 9]. These estimates still use (7.36), combined with the fact that, in equations (5.63), (5.67), the size of the terms of nonzero degree in $\Lambda^{*}\left(T^{*} S\right)$ is dominated by $C p$. These estimates still give (7.35).

By (7.34), (7.35), we get (7.28). The proof of our proposition is completed.

By (7.28), for $p \in \mathbf{N}, 1 \leqslant t \leqslant p$,

$$
\left|p^{-n} \theta_{1 / \sqrt{p}} \operatorname{Tr}_{\mathrm{s}}\left[\widetilde{G}_{t / p^{2}}\left(L_{t / p^{2}}^{F_{p^{\prime}}}\right)\right]\right| \leqslant C \exp \left(-c p^{2} / t\right) \leqslant C \exp \left(-\frac{1}{2} c(t+p)\right) .
$$

By (7.27), (7.39), to establish (7.18), we are left to prove that, for $1 \leqslant t \leqslant p$,

$$
\left|p^{-n} \theta_{1 / \sqrt{p}} \operatorname{Tr}_{\mathrm{s}}\left[\widetilde{F}_{t / p^{2}}\left(L_{t / p^{2}}^{F_{p^{\prime}}}\right)\right]\right| \leqslant C .
$$

Using finite propagation speed for hyperbolic equations, [27, §7.8], [46, §4.4], for $x \in X$, the support of the smooth kernel $\widetilde{F}_{t / p^{2}}\left(L_{t / p^{2}} F_{p^{\prime}}\right)$ is included in the open ball $B^{X}(0, \epsilon / 2)$. Therefore, the proof of $(7.40)$ can be localized. Now, we proceed as in the proof of Theorem 6.5. There, the proof was based on a choice of local coordinates near $x \in X$. The support condition given before shows that we can safely replace $X$ by $T_{x} X$, while extending the operator $L_{t / p^{2}}^{F_{p^{\prime}}}$ outside the ball $B^{T_{x} X}(0, \epsilon / 2)$ to the full $T_{x} X$, so that it has essentially the same structure as $L_{t / p^{2}}^{F_{p^{\prime}}}$.

We consider equations (5.63), (5.67) for $L_{t / p^{2}}^{F_{p}}$. In $\Lambda_{t / p^{2}}$, the component of degree 0 in $\mathbf{R}[z] \widehat{\otimes} \Lambda^{\cdot}\left(T^{*} S\right)$ is just $\frac{t}{4 p^{2}} \Theta$. By $(7.21)$, for $p \in \mathbf{N}$ large enough, $\frac{\rho_{p} \Theta}{p^{2}}-a$ has a positive lower bound. 
We will now proceed as in the proof of Theorem 6.5, doing the rescaling on the coordinate $Y$ near $x$ and also the same Getzler rescaling on the Clifford variables, while not performing any rescaling on the coordinates in $G_{\mathbf{C}}$. Equivalently, we still treat $F_{p}$ as an ordinary flat Hermitian vector bundle. By equation (3.17) in Theorem 3.1, proceeding as in the proof of (4.21), if $A \in \mathfrak{g}_{\mathbf{C}}$, we get

$$
\frac{1}{p}\left\|\left.L_{A}\right|_{H^{(0,0)}\left(N, L^{p}\right)}\right\| \leqslant C|A| .
$$

While, in the proof of Theorem 4.4, the estimate in (7.41) was enough to obtain the spectral gap in (4.18), (4.19), here, the rescaling on the Getzler variables makes certain terms bigger. As an example, after the Getzler rescaling, the leading term as $p \rightarrow+\infty$ in $\frac{t}{p} \widehat{c}_{1 / p}\left(e_{i}\right) \widehat{c}_{1 / p}\left(e_{j}\right)$ is given by $t \widehat{e}^{i} \wedge \widehat{e}^{j}$, which is a nilpotent operator, whose size for $1 \leqslant t \leqslant p$ is not uniformly controlled. To take advantage of the uniform positivity of $\frac{\rho_{p} \Theta}{p^{2}}-a$, as in $[17, \S 11(\mathrm{k})]$, we introduce a systems of Hilbert norms with weights. Namely, for $\lambda>0$ small enough, $s \in C^{\infty}\left(T_{x} X, \Lambda^{\cdot}\left(T_{x}^{*} X\right) \otimes F_{p, x}\right)$, set

$$
|s|_{\lambda}^{2}=\left|\lambda^{2 N^{\Lambda^{*}\left(T^{*} X\right)}} s\right|_{L_{2}}^{2}
$$

With respect to the norm ||$_{\lambda}$, if $f \in T^{*} X$, the norm of the operator $f \wedge$ is now $\lambda|f|$. Of course the contribution of operators like $i_{e}$ is increased by the factor $1 / \lambda$, but such operators always have an extra factor like $1 / \sqrt{p}$ which makes them small in the end. Ultimately, when picking up the proper $\lambda>0$, for $t \geqslant 1$, we get a positive lower bound

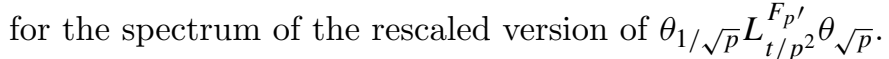

Using a contour integral formula then leads easily to a proof of (7.40). This completes the proof of equation (7.15).

We claim that we can now establish the full second equation in (7.4), which requires refining (7.16) to

$$
\int_{1}^{p} p^{-n-1} \psi_{1 / \sqrt{p}} h^{\wedge}\left(A^{\prime}, g_{t / p^{2}}^{\Omega \cdot\left(X, F_{p} \mid X\right)}\right) \frac{d t}{t}=\int_{1}^{+\infty} \pi_{*} d_{t / 4} \frac{d t}{t}+\mathcal{O}\left(p^{-1}\right) .
$$

We still use equation (7.27). By (7.39), as $p \rightarrow+\infty$,

$$
\int_{1}^{p} p^{-n} e^{-a t / 4} \theta_{1 / \sqrt{p}} \operatorname{Tr}_{\mathrm{s}}\left[\widetilde{G}_{t / p^{2}}\left(L_{t / p^{2}}^{F_{p^{\prime}}}\right)\right] \frac{d t}{t}=\mathcal{O}\left(e^{-c p}\right) .
$$

Using previous arguments, given $t \geqslant 1$, as $p \rightarrow+\infty, p^{-n} \theta_{1 / \sqrt{p}} \operatorname{Tr}_{\mathrm{s}}\left[\widetilde{H}_{t / p^{2}}\left(L_{t / p^{2}}^{F_{p^{\prime}}}\right)\right]$ has an asymptotic expansion that is uniform when $t \geqslant 1$ remains bounded. By proceeding as in the proof of Theorem 6.5 , for $p \in \mathbf{N}^{*}, 1 \leqslant t \leqslant p$, over compact subsets of $S$,

$$
\left|p^{-n} \frac{1}{\sqrt{p}} \psi_{1 / \sqrt{p}} \operatorname{Tr}_{\mathrm{s}}\left[\widetilde{F}_{t / p^{2}}\left(L_{t / p^{2}}^{F_{p^{\prime}}}\right)\right]^{z}-e^{a t / 4} \pi_{*} c_{t / 4}\right| \leqslant C \frac{t}{p} .
$$


By (7.45), over compact subsets of $S$, we get

$$
\begin{aligned}
& \left|\int_{1}^{p}\left(e^{-a t / 4} p^{-n} \frac{1}{\sqrt{p}} \psi_{1 / \sqrt{p}} \operatorname{Tr}_{\mathrm{s}}\left[\widetilde{F}_{t / p^{2}}\left(L_{t / p^{2}}^{F_{p^{\prime}}}\right)\right]^{z}-\pi_{*} c_{t / 4}\right) \frac{d t}{t}\right| \\
& \quad \leqslant C p^{-1} \int_{1}^{+\infty} e^{-a t / 4} d t .
\end{aligned}
$$

By (7.27), (7.39), and (7.46), we get

$$
\left|\int_{1}^{p}\left(p^{-n} \frac{1}{\sqrt{p}} h\left(A^{\prime}, g^{\Omega\left(X, F_{p} \mid X\right)}\right)-p_{*} c_{t / 4}\right) \frac{d t}{t}\right| \leqslant \frac{C}{p} .
$$

By proceeding as in (5.44) and in the proof of Theorem 6.5, from (7.47), we get (7.43). This completes the proof of Theorem 7.1.

Remark 7.3. Let $\left(F^{\prime}, \nabla^{F^{\prime}}, g^{F^{\prime}}\right)$ be a flat Hermitian vector bundle on $M$. One verifies easily that, if we replace $F_{p}$ by $F_{p}^{\prime}=F_{p} \otimes F^{\prime}$ in the left-hand side of $(7.1)$, then the right-hand side of (7.1) should be multiplied by $\operatorname{dim} F^{\prime}$. Similarly, if $L^{\prime}$ is a holomorphic vector bundle on $N$ to which the action of $G$ also lifts, we could as well deal with $L^{p} \otimes L^{\prime}$, the only effect being to multiply the right-hand side of (7.1) by $\operatorname{dim} L^{\prime}$.

\subsection{Compatibility of Theorem 7.1 to known results}

We will check the compatibility of Theorem 7.1 to known results on analytic torsion forms.

By equation (5.46) in Theorem 5.16, we know that, for $p \in \mathbf{N}$ large enough,

$$
d \mathcal{T}_{h}\left(T^{H} M, g^{T X}, \nabla^{F_{p}}, g^{F_{p}}\right)=\pi_{*}\left[e\left(T X, \nabla^{T X}\right) h\left(\nabla^{F_{p}}, g^{F_{p}}\right)\right] .
$$

By equation (6.1) in Proposition 6.1, and by equation (7.1) in Theorem 7.1, when taking the asymptotics of (7.48) as $p \rightarrow+\infty$, we recover equation (2.77) in Theorem 2.12.

Moreover, by Ma [33, Theorem 0.1], $\mathcal{T}_{h}\left(T^{H} M, g^{T X}, \nabla^{F_{p}}, g^{F_{p}}\right)$ satisfies natural compatibility relations under compositions of proper submersions. The form $\pi_{*} W$ in (7.1) should verify corresponding compatibility relations. These relations were proved directly in Theorems 2.14 and 2.16 .

\subsection{Rigidity of the class of $\pi_{*} W$}

As we saw in Remark 2.13, if $\widehat{\theta}^{\mathfrak{p}}$ is nondegenerate, the infinitesimal variation on $\pi_{*} W$ in $\Omega(S) / d \Omega^{-1}(S)$ can be explicitly calculated. We will now obtain a corresponding global result.

Let $h, h^{\prime} \in \mathcal{S}$. The class $\widetilde{\gamma}\left(h, h^{\prime}\right)$ on $M$ was defined in Remark 2.8 .

We assume that $h, h^{\prime}$ are such that the corresponding $\widehat{\theta}^{\mathfrak{p}}, \widehat{\theta}^{\mathfrak{p}^{\prime}}$ are both nondegenerate. We denote with a ${ }^{\prime}$ the objects considered above that are attached to $T^{H} M, g^{T X}, h^{\prime}$.

Theorem 7.4. The following identity holds:

$$
\pi_{*}\left(W^{\prime}-W\right)=\pi_{*}\left[e\left(T X, \nabla^{T X}\right) \tilde{\gamma}\left(h, h^{\prime}\right)\right] \quad \text { in } \Omega(S) / d \Omega^{-1}(S) .
$$

If $\operatorname{dim} X$ is odd, then

$$
\pi_{*} W=\pi_{*} W^{\prime} \quad \text { in } H^{\cdot}(S, \mathbf{R})
$$


Proof. To establish (7.49), we use Theorems 5.17 and 7.1, together with the fact that, by Proposition 6.1, as $p \rightarrow+\infty$,

$$
p^{-n-1} \psi_{1 / \sqrt{p}} \widetilde{h}\left(\nabla^{F_{p}}, g^{F_{p}}, g^{F_{p^{\prime}}}\right)=\tilde{\gamma}\left(h, h^{\prime}\right)+\mathcal{O}\left(p^{-1}\right) .
$$

When $\operatorname{dim} X$ is odd, (7.49) reduces to (7.50). The proof of our theorem is completed.

Remark 7.5. In general, one cannot interpolate between $h$ and $h^{\prime}$ by a family $\ell \in[0,1] \rightarrow$ $h_{\ell} \in \mathcal{S}$ such that the corresponding $\widehat{\theta}_{\ell}^{\mathfrak{p}}$ are nondegenerate. If this is the case, then Theorem 7.4 is trivial. We do not know how to prove this result in general without using Theorem 7.1.

By Theorems 2.12 and 7.4, if $\operatorname{dim} X$ is odd, the form $\pi_{*} W$ is closed, and its cohomology class does not depend on the metric data $T^{H} M, g^{T X}, h$ as long as $\widehat{\theta}^{\mathfrak{p}}$ is nondegenerate.

Let $\ell \in[0,1] \rightarrow f_{\ell} \in \mathcal{F}$ be a family of flat connections on $P_{G}$. Let $\ell \in[0,1] \rightarrow \theta_{\ell}^{\mathfrak{g}}$ denote the corresponding family of flat connection forms on $P_{G}$. Recall that the associated class of forms $\tilde{\gamma}$ on $M$ was defined in Remark 2.18. We assume that, for $\ell \in[0,1], \widehat{\theta}_{\ell}^{\mathfrak{p}}$ is nondegenerate. Let $\ell \in[0,1] \rightarrow W_{\ell}$ be the associated family of forms on $M$.

Theorem 7.6. The following identity holds:

$$
\pi_{*}\left(W_{1}-W_{0}\right)^{(\geqslant 2)}=\pi_{*}\left[e\left(T X, \nabla^{T X}\right) \tilde{\gamma}^{(\geqslant 2)}\right] \text { in } \Omega(S) / d \Omega^{-1}(S) .
$$

If $\operatorname{dim} X$ is odd, then

$$
\pi_{*} W_{1}^{(\geqslant 2)}=\pi_{*} W_{0}^{(\geqslant 2)} \text { in } H^{\cdot}(S, \mathbf{R}) .
$$

Proof. We use the notation of Remark 5.3. From the explicit formulae in [15, Definition 2.4 and Theorem 2.5], and proceeding as in the proof of Proposition 6.1, one finds easily that, as $p \rightarrow+\infty$,

$$
p^{-n-1} \psi_{1 / \sqrt{p}} \widetilde{h}\left(\nabla_{\ell}^{F_{p}}, g^{F_{p}}\right)=\tilde{\gamma}+\mathcal{O}\left(p^{-1}\right) .
$$

By Theorems 5.19 and 7.1, and using (7.54), we get (7.52) and (7.53).

\subsection{The asymptotics of the $\Gamma$-torsion}

We make the same assumptions as in $\S 6.6$, and we use the corresponding notation. In particular, $S$ is reduced to a point. We assume that $\widehat{\theta}^{\mathfrak{p}}$ is nondegenerate. For $p \in \mathbf{N}$ large enough, the $\Gamma$-torsion $\mathcal{T}_{h}^{\Gamma}\left(g^{T X}, \nabla^{F_{p}}, g^{F_{p}}\right) \in \mathbf{R}$ is well defined.

We still define the form $W$ on $X$ as in Definition 2.11. Since $S$ is a point, $\pi_{*} W \in \mathbf{R}$.

Theorem 7.7. As $p \rightarrow+\infty$,

$$
p^{-n-1} \mathcal{T}_{h}^{\Gamma}\left(g^{T X}, \nabla^{F_{p}}, g^{F_{p}}\right)=\pi_{*} W+\mathcal{O}\left(p^{-1}\right) .
$$

Proof. The proof is essentially the same as the proof of Theorem 7.1. However, the arguments in the proof of (7.5)-(7.8) have to be adequately modified. If $z, z^{\prime} \in \widehat{X}, A \in$ $\operatorname{Hom}\left(\widehat{\pi}^{*}\left(\Lambda^{*}\left(T^{*} X\right) \otimes_{\mathbf{R}} \widehat{\pi}^{*} F_{p}\right)_{z}, \widehat{\pi}^{*}\left(\Lambda^{*}\left(T^{*} X\right) \otimes_{\mathbf{R}} \widehat{\pi}^{*} F_{p}\right)_{z^{\prime}}\right)$, set

$$
|A|=\operatorname{Tr}\left[A^{*} A\right]^{1 / 2} \text {. }
$$


For $z \in \widehat{X}$, we equip the vector space

$$
L_{2}\left(\widehat{X}, \operatorname{Hom}\left(\widehat{\pi}^{*}\left(\Lambda^{*}\left(T^{*} X\right) \otimes_{\mathbf{R}} \widehat{\pi}^{*} F_{p}\right)_{z}, \widehat{\pi}^{*}\left(\Lambda^{*}\left(T^{*} X\right) \otimes_{\mathbf{R}} \widehat{\pi}^{*} F_{p}\right) .\right)\right)
$$

with the corresponding $L_{2}$ norm. Let $P_{p, t}\left(z, z^{\prime}\right)$ be the smooth kernel associated with $\exp \left(-t D_{p}^{\widehat{X}, 2}\right)$. Then

$$
\operatorname{Tr}\left[P_{p, t}(z, z)\right]=\left\|P_{p, t / 2}(z, \cdot)\right\|_{L_{2}}^{2} .
$$

We can rewrite $(7.57)$ in the form

$$
\operatorname{Tr}\left[P_{p, t}(z, z)\right]=\left\|\exp \left(-t D_{p}^{\widehat{X}, 2} / 2\right) \delta_{z}\right\|_{L_{2}}^{2} .
$$

By (7.58), $\operatorname{Tr}\left[P_{p, t}(z, z)\right]$ decreases with $t$. By Theorem 4.4 and Remark 4.5, we get an analogue of (7.5), (7.6); i.e., given $\alpha>0$, for $t \geqslant \alpha$,

$$
\operatorname{Tr}^{\Gamma}\left[\exp \left(-t D_{p}^{\widehat{X}, 2}\right)\right] \leqslant \exp \left(-\left(c p^{2}-C\right)(t-\alpha)\right) \operatorname{Tr}^{\Gamma}\left[\exp \left(-\alpha D_{p}^{\widehat{X}, 2}\right)\right] .
$$

The proof continues as the proof of Theorem 7.1.

Remark 7.8. Inspection of the proofs of Theorems 6.5, 7.1, and 7.7 shows easily that there is $c>0$ such that, as $p \rightarrow+\infty$,

$$
\mathcal{T}_{h}\left(g^{T X}, \nabla^{F_{p}}, g^{F_{p}}\right)-\mathcal{T}_{h}^{\Gamma}\left(g^{T X}, \nabla^{F_{p}}, g^{F_{p}}\right)=\mathcal{O}\left(e^{-c p}\right) .
$$

\section{Asymptotic torsion and orbital integrals}

The purpose of this section is to demonstrate the compatibility of the results of the previous sections to the evaluation of semisimple elliptic orbital integrals in [11]. More precisely, if $G$ is a connected reductive group and $K$ is a maximal compact subgroup, we take $X$ to be the symmetric space $G / K$ or a compact quotient $\Gamma \backslash G / K$ by a discrete torsion-free cocompact subgroup $\Gamma \subset G$, and $S$ to be a point. In this case $\pi_{*} W$ can be evaluated more explicitly. Moreover, the asymptotics of the orbital integrals are evaluated explicitly so as to recover certain results of $\S 6$. Also, conditions are given so that $\widehat{\theta}^{\mathfrak{p}}$ is nondegenerate.

This section is organized as follows. In $\S 8.1$, the symmetric space $X=G / K$ is considered.

In $\S 8.2$, we introduce the Casimir operator on $G$.

In $\S 8.3$, the case where $G=K_{\mathbf{C}}$ is briefly considered.

In $\S 8.4$, a formula for $D^{X, 2}$ is given in terms of the Casimir operator.

In $\S 8.5$, using the results of [11], certain orbital integrals associated with $\exp \left(-t D^{X, 2} / 2\right)$ are evaluated. The integrand of the Ray-Singer analytic torsion of locally symmetric spaces that are associated with our flat bundles $F$ is still shown to vanish, except for the restricted list found by Moscovici and Stanton [40], [11, Remark 7.9.2], who had considered the case where $F$ is unitarily flat.

In $\S 8.6$, the forms $d_{t}$ are shown to vanish for the symmetric spaces not included in the list of $[40]$.

In $\S 8.7, \pi_{*} W \in \mathbf{R}$ is computed in the case where $G=\mathrm{SL}_{2}(\mathbf{C})$, and results of Müller [43] are recovered in the case where $G=\mathrm{SL}_{2}(\mathbf{C})$. 
In $\S 8.8$, if $\lambda$ is a weight for $U$, and if $N=N_{\lambda}$ is the corresponding coadjoint orbit of $U$, necessary and sufficient conditions on $\lambda$ are given under which $\widehat{\theta}^{\mathfrak{p}}$ is nondegenerate.

Finally, in $\S 8.9$, we obtain the asymptotics as $p \rightarrow+\infty$ of certain orbital integrals associated with the heat kernel of $D^{X, 2}$. Of special relevance is the integrand of the classical Ray-Singer analytic torsion. The forms $d_{t}, e_{t}$ that were defined in Definition 2.9 are used to express the asymptotics.

\subsection{Reductive groups and symmetric spaces}

We use the notation of $\S 1.1$. Set

$$
m=\operatorname{dim} \mathfrak{p}, \quad m^{\prime}=\operatorname{dim} \mathfrak{k} .
$$

Let $\theta^{\mathfrak{g}}$ be the canonical left-invariant 1 -form on $G$ with values in $\mathfrak{g}$, and let $\theta^{\mathfrak{p}}, \theta^{\mathfrak{k}}$ be its components in $\mathfrak{p}, \mathfrak{k}$, so that

$$
\theta^{\mathfrak{g}}=\theta^{\mathfrak{p}}+\theta^{\mathfrak{k}} .
$$

Let $X=G / K$ be the associated symmetric space. Then $p: G \rightarrow X=G / K$ is a $K$-principal bundle, equipped with the connection form $\theta^{\mathfrak{k}}$. As in (1.38), (1.39), the curvature $\Theta^{\mathfrak{k}}$ of this connection is given by

$$
\Theta^{\mathfrak{k}}=-\frac{1}{2}\left[\theta^{\mathfrak{p}}, \theta^{\mathfrak{p}}\right]=-\theta^{\mathfrak{p}, 2} .
$$

Let $e_{1}, \ldots, e_{m}$ be a basis of $\mathfrak{p}$, and let $e^{1}, \ldots, e^{m}$ be the corresponding dual basis of $\mathfrak{p}^{*}$. We can rewrite (8.3) in the form

$$
\Theta^{\mathfrak{k}}=-\frac{1}{2} \sum_{1 \leqslant i, j \leqslant m} e^{i} \wedge e^{j} \otimes\left[e_{i}, e_{j}\right] .
$$

We use the notation of $\S 1.6$. Making $M=X, P_{G}=G$, the assumptions of $\S 1.6$ are verified. Indeed, $\theta^{\mathfrak{g}}$ defines a flat connection on $P_{G}$. Also, $G \times{ }_{G} G / K=X$ has a canonical tautological section over $X$. Equivalently, the reduction of the principal bundle $p: G \rightarrow X$ to a $K$-bundle is given tautologically. We can then apply to the present situation the arguments and results of the previous sections.

If $E$ is a finite-dimensional real Euclidean vector space, and if $\rho^{E}: K \rightarrow \operatorname{Aut}(E)$ is a representation of $K$ by isometries of $E$, then $F=G \times_{K} E$ is an Euclidean vector bundle on $X$, which is naturally equipped with an Euclidean connection $\nabla^{F}$. Also, we may as well assume that $E$ is a complex Hermitian vector space, and that $F$ is a Hermitian vector bundle equipped with a unitary connection.

If the representation $\rho^{E}$ is induced by a representation $G \rightarrow \operatorname{Aut}(E)$ that is still denoted $\rho^{E}$, the map $(g, v) \in G \times{ }_{K} E \rightarrow \rho^{E}(g) v \in E$ gives the canonical identification

$$
G \times{ }_{K} E=X \times E .
$$

In this case, the vector bundle $F$ is also equipped with a canonical flat connection $\nabla^{F, f}$, and moreover,

$$
\nabla^{F, f}=\nabla^{F}+\rho^{E} \theta^{\mathfrak{p}}
$$

As in (4.3),

$$
\omega\left(\nabla^{F, f}, g^{F}\right)=-2 \rho^{E} \theta^{\mathfrak{p}}
$$


Note the change of notation with respect to the previous sections, since $\nabla^{F}, \nabla^{F, u}$ have become $\nabla^{F, f}, \nabla^{F}$.

If $\rho^{E}: U \rightarrow \operatorname{Aut}(E)$ is a complex representation of $U$, there is a Hermitian metric $g^{E}$ on $E$ that is such that the representation is unitary. As we saw in $\S 1.1, \rho^{E}$ extends uniquely to a representation of $G$, so that we are in the situation we considered above.

Observe that $G$ acts on $\mathfrak{g}$ by the adjoint representation. The tangent bundle $T X$ is given by

$$
T X=G \times_{K} \mathfrak{p} .
$$

Put

$$
N=G \times{ }_{K}^{\mathfrak{k}}
$$

Then

$$
T X \oplus N=G \times{ }_{K} \mathfrak{g} .
$$

By the above, $T X, N$ are equipped with Euclidean connections $\nabla^{T X}, \nabla^{N}$. Also, the connection $\nabla^{T X}$ is the Levi-Civita connection of $T X$. Moreover, $T X \oplus N$ is canonically flat, and it can be identified with the trivial vector bundle $\mathfrak{g}$. Comparing with (1.33), (1.36), we get

$$
T X \oplus N=\mathfrak{g}_{r}, \quad T X=\mathfrak{p}_{r}, \quad N=\mathfrak{k}_{r} .
$$

By (8.6), we get

$$
\nabla^{T X \oplus N, f}=\nabla^{T X \oplus N}+\operatorname{ad}\left(\theta^{\mathfrak{p}}\right)
$$

By (8.12), we get

$$
\omega\left(\nabla^{T X \oplus N, f}, g^{T X \oplus N}\right)=-2 \operatorname{ad}\left(\theta^{\mathfrak{p}}\right) .
$$

Let $R^{T X}$ be the curvature of $\nabla^{T X}$. If $a, b, c \in T X$, then

$$
R^{T X}(a, b) c=-[[a, b], c] .
$$

Note that $\theta^{\mathfrak{p}}$ can be identified with the identity section of $T^{*} X \otimes T X$. Therefore, if $U \in T X$

$$
\nabla_{U}^{T X \otimes \mathfrak{g}_{r}, u} \theta^{\mathfrak{p}}=0
$$

\subsection{The Casimir operator of $G$}

Let $C^{\mathfrak{g}} \in U \mathfrak{g}$ be the Casimir of $\mathfrak{g}$. If $e_{1}, \ldots, e_{m}$ is an orthonormal basis of $\mathfrak{p}$, and $e_{m+1}, \ldots, e_{m+m^{\prime}}$ is an orthonormal basis of $\mathfrak{k}$, then

$$
C^{\mathfrak{g}}=-\sum_{i=1}^{m} e_{i}^{2}+\sum_{i=m+1}^{m+m^{\prime}} e_{i}^{2}
$$

Set

$$
C^{\mathfrak{g}, H}=-\sum_{i=1}^{m} e_{i}^{2}, \quad C^{\mathfrak{k}}=\sum_{i=m+1}^{m+m^{\prime}} e_{i}^{2},
$$

so that

$$
C^{\mathfrak{g}}=C^{\mathfrak{g}, H}+C^{\mathfrak{k}}
$$


As the notation indicates, $C^{\mathfrak{k}}$ is the Casimir of $\mathfrak{k}$ that is associated with $\left.B\right|_{\mathfrak{k}}$. By [11, equation (2.5.7)],

$$
\left[C^{\mathfrak{g}, H}, C^{\mathfrak{k}}\right]=0 .
$$

Let $E$ be a complex vector space, and let $\rho^{E}$ be a representation of $G$ in $\operatorname{Aut}(E)$. Let $C^{\mathfrak{g}, E} \in \operatorname{End}(E)$ be the Casimir of $\mathfrak{g}$ which is associated with the representation $\rho^{E}$. Namely,

$$
C^{\mathfrak{g}, E}=\rho^{E} C^{\mathfrak{g}} .
$$

Set

$$
C^{\mathfrak{g}, H, E}=\rho^{E} C^{\mathfrak{g}, H}, \quad C^{\mathfrak{k}, E}=\rho^{E} C^{\mathfrak{k}} .
$$

Then

$$
C^{\mathfrak{g}, E}=C^{\mathfrak{g}, H, E}+C^{\mathfrak{k}, E}
$$

\subsection{The complexification of compact Lie groups}

In this subsection, we assume that $G$ is the complexification $K_{\mathbf{C}}$ of the compact connected Lie group $K$. The Lie algebra $\mathfrak{g}$ of $G$ is given by

$$
\mathfrak{g}=i \mathfrak{k} \oplus \mathfrak{k},
$$

so that, with respect to the notation in (1.1),

$$
\mathfrak{p}=i \mathfrak{k} .
$$

If \langle\rangle is a $K$-invariant scalar product on $\mathfrak{k}$, then -\langle\rangle extends to a real symmetric bilinear form $B$ on $\mathfrak{g}$ which has the properties indicated in $\S 1$.1. In what follows, we identify $i$ with the obvious complex structure of $\mathfrak{g}$, which exchanges $\mathfrak{p}$ and $\mathfrak{k}$.

Let $\kappa^{\mathfrak{k}} \in \Lambda^{3}\left(\mathfrak{k}^{*}\right)$ be such that, if $a, b, c \in \mathfrak{k}$,

$$
\kappa^{\mathfrak{k}}(a, b, c)=B([a, b], c)=-\langle[a, b], c\rangle .
$$

Then $\kappa^{\mathfrak{k}}$ is Ad-invariant. It induces a closed left- and right-invariant 3-form on $K$.

Let $\kappa^{\mathfrak{p}} \in \Lambda^{3}\left(\mathfrak{p}^{*}\right)$ be such that, if $a, b, c \in \mathfrak{p}$,

$$
\kappa^{\mathfrak{p}}(a, b, c)=-\kappa^{\mathfrak{k}}(i a, i b, i c) .
$$

By (8.25), (8.26), we get

$$
\kappa^{\mathfrak{p}}(a, b, c)=B([a, b], i c)
$$

Then $\kappa^{\mathfrak{p}}$ is $K$-invariant.

Proposition 8.1. The form $\kappa^{\mathfrak{p}}$ descends to a closed 3-form on $X$ which is parallel with respect to $\nabla^{T X}$.

Proof. Since $\kappa^{\mathfrak{k}}$ is $K$-invariant, it descends to a 3 -form on $X$ which is parallel with respect to $\nabla^{T X}$. Therefore it is also a closed form. 
Observe that $\operatorname{ad}\left(i \theta^{\mathfrak{p}}\right)$ acts as an antisymmetric endomorphism of $\mathfrak{p}$ into itself. Moreover,

$$
\operatorname{ad}\left(i \theta^{\mathfrak{p}}\right)^{2}=-\operatorname{ad}\left(\theta^{\mathfrak{p}}\right)^{2}
$$

Equation (8.14) can be written in the form

$$
R^{T X}=\operatorname{ad}\left(i \theta^{\mathfrak{p}}\right)^{2} .
$$

By (8.15), if $U \in T X$,

$$
\nabla_{U}^{T X} \operatorname{ad}\left(i \theta^{\mathfrak{p}}\right)=0
$$

Proposition 8.2. The Pontryagin forms of $\left(T X, \nabla^{T X}\right)$ are concentrated in degree 0 . Also,

$$
e\left(T X, \nabla^{T X}\right)=0 .
$$

Proof. We use equation (8.29), together with the fact that $\operatorname{ad}\left(i \theta^{\mathfrak{p}}\right)$ acts as an antisymmetric endomorphism of $T X$ into itself. By proceeding as in [18, proof of Proposition 1.3], our proposition follows.

Remark 8.3. Here is another proof of Proposition 8.2. First, we consider the Chern character form. By [11, Proposition 7.1.1],

$$
\operatorname{ch}\left(T X, \nabla^{T X}\right)+\operatorname{ch}\left(N, \nabla^{N}\right)=2 m^{\prime} .
$$

On the other hand, $i: T X \rightarrow N$ is a parallel morphism. Therefore,

$$
\operatorname{ch}\left(T X, \nabla^{T X}\right)=\operatorname{ch}\left(N, \nabla^{N}\right) .
$$

By (8.32), (8.33), we get

$$
\operatorname{ch}\left(T X, \nabla^{T X}\right)=m^{\prime}
$$

The same argument can be used for the other Pontryagin classes. Let us now consider the case of the Euler class. Recall that $\Theta^{\mathfrak{k}}$ is given by (8.4). Set

$$
\widehat{\Theta}^{\mathfrak{k}}=-\frac{1}{2} \sum_{1 \leqslant i, j \leqslant m} \widehat{e}^{i} \wedge \widehat{e}^{j} \otimes\left[e_{i}, e_{j}\right] .
$$

Then

$$
\frac{1}{2} \sum_{1 \leqslant i, j \leqslant m}\left\langle e_{i}, R^{T X} e_{j}\right| \widehat{e}^{i} \widehat{e}^{j}=-\left\langle\Theta^{\mathfrak{k}}, \widehat{\Theta}^{\mathfrak{k}}\right\rangle .
$$

In (8.36), the scalar product is taken of the $\mathfrak{k}$ components of $\Theta, \widehat{\Theta}$.

Let $\widehat{\kappa}^{\mathfrak{p}}$ be the obvious analogue of $\kappa^{\mathfrak{p}}$. By $(8.27)$, (8.36), we get

$$
\frac{1}{2} \sum_{1 \leqslant i, j \leqslant m}\left\langle e_{i}, R^{T X} e_{j}\right| \widehat{e}^{i} \wedge \widehat{e}^{j}=-i_{i \Theta^{\mathfrak{k}} \widehat{\kappa}^{\mathfrak{p}}} .
$$

By (1.44), (8.37), we get

$$
e\left(T X, \nabla^{T X}\right)=\int^{\widehat{B}} \exp \left(\frac{1}{2} i_{i \Theta^{\mathfrak{k}} \widehat{\kappa}^{\mathfrak{p}}}\right) .
$$

Since the exponential in (8.38) can never be of top degree in $\Lambda^{\cdot}\left(\widehat{T^{*} X}\right)$, we get $(8.31)$. 


\subsection{The Weitzenböck formula on $X$}

We assume again that $G$ is an arbitrary connected reductive group. Let $E$ be a complex Hermitian vector space, and let $\rho^{E}: U \rightarrow \operatorname{Aut}(E)$ be a unitary representation of $U$. As we saw in $\S 8.1, E$ descends to a vector bundle $F$ equipped with a flat connection $\nabla^{F, f}$ and with a unitary connection $\nabla^{F}$.

By (4.11), (8.15), we get

$$
\begin{aligned}
D^{X, 2}= & -\Delta^{X, u}+\frac{K^{X}}{4}-\frac{1}{8}\left\langle R^{T X}\left(e_{i}, e_{j}\right) e_{k}, e_{\ell}\right\rangle c\left(e_{i}\right) c\left(e_{j}\right) \widehat{c}\left(e_{k}\right) \widehat{c}\left(e_{\ell}\right) \\
& +\rho^{E}\left|\underline{\theta}^{\mathfrak{p}}\right|^{2}-\frac{1}{2}\left(c\left(e_{i}\right) c\left(e_{j}\right)-\widehat{c}\left(e_{i}\right) \widehat{c}\left(e_{j}\right)\right) \rho^{E} \theta^{\mathfrak{p}, 2}\left(e_{i}, e_{j}\right) .
\end{aligned}
$$

The tensors that follow $-\Delta^{X, u}$ in (8.39) are parallel with respect to $\nabla^{\Lambda^{\prime}\left(T^{*} X\right) \otimes_{\mathbf{R}} F}$.

By [11, equation (2.6.8)], or by (8.14), we get

$$
K^{X}=\operatorname{Tr}^{\mathfrak{p}}\left[C^{\mathfrak{k}, \mathfrak{p}}\right] .
$$

Set

$$
\underline{D}^{X}=c\left(e_{i}\right) \nabla_{e_{i}}^{\Lambda \cdot\left(T^{*} X\right) \otimes \mathbf{R} F} .
$$

Then $\underline{D}^{X}$ is a standard Dirac operator on $X$. By (4.10), (8.7), we get

$$
D^{X}=\underline{D}^{X}+\sum_{i=1}^{m} \widehat{c}\left(e_{i}\right) \rho^{E}\left(e_{i}\right)
$$

By (8.15), (8.42), we obtain

$$
D^{X, 2}=\underline{D}^{X, 2}+\left(\sum_{i=1}^{m} \widehat{c}\left(e_{i}\right) \rho^{E}\left(e_{i}\right)\right)^{2} .
$$

Also, the two operators in the right-hand side of (8.43) commute. Then (8.43) can be rewritten in the form

$$
D^{X, 2}=\underline{D}^{X, 2}+\sum_{i=1}^{m} \rho^{E}\left(e_{i}\right)^{2}+\frac{1}{2} \sum_{1 \leqslant i, j \leqslant m} \widehat{c}\left(e_{i}\right) \widehat{c}\left(e_{j}\right) \rho^{E}\left(\left[e_{i}, e_{j}\right]\right) .
$$

By (8.17), equation (8.44) is equivalent to

$$
D^{X, 2}=\underline{D}^{X, 2}-C^{\mathfrak{g}, H, E}+\frac{1}{2} \sum_{1 \leqslant i, j \leqslant m} \widehat{c}\left(e_{i}\right) \widehat{c}\left(e_{j}\right) \rho^{E}\left(\left[e_{i}, e_{j}\right]\right) .
$$

Let $C^{\mathfrak{g}, X}$ be the Casimir operator of $G$ acting on $C^{\infty}\left(X, \Lambda^{\cdot}\left(T^{*} X\right) \otimes_{\mathbf{R}} F\right)$. Let $B^{*}\left(\kappa^{\mathfrak{g}}, \kappa^{\mathfrak{g}}\right) \in \mathbf{R}$ be the constant defined in [11, §2.6]. Its precise value is irrelevant here. Set

$$
\mathcal{L}^{X, F}=\frac{1}{2} C^{\mathfrak{g}, X}+\frac{1}{8} B^{*}\left(\kappa^{\mathfrak{g}}, \kappa^{\mathfrak{g}}\right) .
$$

Proposition 8.4. The following identity holds:

$$
\frac{D^{X, 2}}{2}=\mathcal{L}^{X, F}-\frac{1}{2} C^{\mathfrak{g}, E}-\frac{1}{48} \operatorname{Tr}^{\mathfrak{k}}\left[C^{\mathfrak{k}, \mathfrak{k}}\right]-\frac{1}{16} \operatorname{Tr}^{\mathfrak{p}}\left[C^{\mathfrak{k}, \mathfrak{p}}\right] .
$$


Proof. Let $S^{\mathfrak{p}}$ be the Hermitian vector space of spinors associated with the Euclidean vector space $\mathfrak{p}$, and let $S^{\mathfrak{p} *}$ be its dual. If $e \in \mathfrak{k}$, set

$$
\widehat{c}\left(\left.\operatorname{ad}(e)\right|_{\mathfrak{p}}\right)=-\frac{1}{4} \sum_{1 \leqslant i, j \leqslant m}\left\langle\operatorname{ad}(e) e_{i}, e_{j}\right) \widehat{c}\left(e_{i}\right) \widehat{c}\left(e_{j}\right) .
$$

By [11, Theorem 7.2.1], using the notation in this reference, we get

$$
\frac{D^{X, 2}}{2}=\mathcal{L}^{X, F}-\frac{1}{8} B^{*}\left(\kappa^{\mathfrak{k}}, \kappa^{\mathfrak{k}}\right)-\frac{1}{2} C^{\mathfrak{k}, S^{\mathfrak{p} *} \otimes E} .
$$

By definition,

$$
C^{\mathfrak{k}, S^{\mathfrak{p} *} \otimes E}=\sum_{i=m+1}^{m+m^{\prime}}\left(\widehat{c}\left(\left.\operatorname{ad}\left(e_{i}\right)\right|_{\mathfrak{p}}\right)+\rho^{E}\left(e_{i}\right)\right)^{2}
$$

By (8.50), we get

$$
C^{\mathfrak{k}, S^{\mathfrak{p} *} \otimes E}=C^{\mathfrak{k}, S^{\mathfrak{p} *}}+C^{\mathfrak{k}, E}+2 \sum_{i=m+1}^{m+m^{\prime}} \widehat{c}\left(\left.\operatorname{ad}\left(e_{i}\right)\right|_{\mathfrak{p}}\right) \rho^{E}\left(e_{i}\right) .
$$

By [11, equations (2.6.7) and (7.8.6)],

$$
B^{*}\left(\kappa^{\mathfrak{k}}, \kappa^{\mathfrak{k}}\right)=\frac{1}{6} \operatorname{Tr}^{\mathfrak{k}}\left[C^{\mathfrak{k}, \mathfrak{k}}\right], \quad C^{\mathfrak{k}, S^{\mathfrak{p} *}}=\frac{1}{8} \operatorname{Tr}^{\mathfrak{p}}\left[C^{\mathfrak{k}, \mathfrak{p}}\right] .
$$

By (8.45), (8.49), and (8.52), we get (8.47). The proof of our proposition is completed.

\subsection{The trivial orbital integrals}

Now, we will evaluate certain trivial orbital integrals, i.e., the orbital integrals associated with the element $1 \in G$. For $t>0$, let $P_{t}\left(x, x^{\prime}\right)$ be the smooth kernel on $X$ which is associated with $\exp \left(-t D^{X, 2} / 2\right)$. By definition, if $x \in X$, the orbital integral associated with $1 \in G$ is given by

$$
\operatorname{Tr}_{\mathrm{s}}{ }^{[1]}\left[\exp \left(-t D^{X, 2} / 2\right)\right]=\operatorname{Tr}_{\mathrm{s}} \Lambda^{*}\left(T^{*} X\right) \otimes \mathbf{R} F\left[P_{t}(x, x)\right]
$$

Of course, the right-hand side of (8.53) does not depend on $x \in X$.

Let $d v_{\mathfrak{p}}$ be the volume form on $\mathfrak{p}$. If $\alpha \in \Lambda^{*}\left(\mathfrak{p}^{*}\right) \otimes o\left(\mathfrak{p}^{*}\right)$, if $\alpha^{(m)}$ is the component of $\alpha$ with top degree $m$, let $\alpha^{\max } \in \mathbf{R}$ be such that

$$
\alpha^{(m)}=\alpha^{\max } d v_{\mathfrak{p}}
$$

First, we extend [11, Theorem 7.8.2], where $E$ was assumed to be trivial. As in [11, equation (5.5.11)], for $Y_{0}^{\mathfrak{k}} \in \mathfrak{k}$, set

$$
J_{1}\left(Y_{0}^{\mathfrak{k}}\right)=\widehat{A}\left(\left.i \operatorname{ad}\left(Y_{0}^{\mathfrak{k}}\right)\right|_{\mathfrak{p}}\right) \widehat{A}^{-1}\left(\left.i \operatorname{ad}\left(Y_{0}^{\mathfrak{k}}\right)\right|_{\mathfrak{k}}\right)
$$


Theorem 8.5. For any $t>0$, the following identity holds:

$$
\begin{aligned}
\operatorname{Tr}_{\mathrm{s}}{ }^{[1]} & {\left[\exp \left(-t D^{X, 2} / 2\right)\right] } \\
= & \frac{1}{(2 \pi t)^{m / 2}} \exp \left(\frac{t}{48} \operatorname{Tr}^{\mathfrak{k}}\left[C^{\mathfrak{k}, \mathfrak{k}}\right]+\frac{t}{16} \operatorname{Tr}^{\mathfrak{p}}\left[C^{\mathfrak{k}, \mathfrak{p}}\right]\right) \\
& \times \int_{\mathfrak{k}} J_{1}\left(Y_{0}^{\mathfrak{k}}\right) \operatorname{Tr}_{\mathrm{s}}{ }^{\prime \cdot\left(\mathfrak{p}^{*}\right) \otimes E}\left[\exp \left(-i \rho^{\Lambda^{\prime}\left(\mathfrak{p}^{*}\right) \otimes E}\left(Y_{0}^{\mathfrak{k}}\right)+\frac{t}{2} C^{\mathfrak{g}, E}\right)\right] \\
& \times \exp \left(-\left|Y_{0}^{\mathfrak{k}}\right|^{2} / 2 t\right) \frac{d Y_{0}^{\mathfrak{k}}}{(2 \pi t)^{m^{\prime} / 2}}=\left[e\left(T X, \nabla^{T X}\right)\right]^{\max } \operatorname{dim} E .
\end{aligned}
$$

Proof. To simplify the notation, we will identify $E$ and $F$. By [11, Theorem 6.1.1], and by Proposition 8.4, we get the first part of (8.56). Moreover,

$$
\begin{aligned}
& \operatorname{Tr}_{\mathrm{S}} \Lambda^{\cdot\left(\mathfrak{p}^{*}\right) \otimes E}\left[\exp \left(-i \rho^{\Lambda \cdot\left(\mathfrak{p}^{*}\right) \otimes E}\left(Y_{0}^{\mathfrak{k}}\right)+\frac{t}{2} C^{\mathfrak{g}, E}\right)\right] \\
& \quad=\left.\operatorname{det}\left(1-\exp \left(i \operatorname{ad}\left(Y_{0}^{\mathfrak{k}}\right)\right)\right)\right|_{\mathfrak{p}} \operatorname{Tr}^{E}\left[\exp \left(-i \rho^{E}\left(Y_{0}^{\mathfrak{k}}\right)+\frac{t}{2} C^{\mathfrak{g}, E}\right)\right] .
\end{aligned}
$$

If $\mathfrak{p}$ is odd dimensional, then 0 is an eigenvalue of $\left.\operatorname{ad}\left(Y_{0}^{\mathfrak{k}}\right)\right|_{\mathfrak{p}}$, so that the integrand in the second line of (8.56) vanishes. So let us assume that $\mathfrak{p}$ is even dimensional. By [11, equation (7.2.15) and Theorem 7.4.1], we get

$$
\begin{aligned}
& \frac{1}{(2 \pi t)^{m / 2}} \int_{\mathfrak{k}} J_{1}\left(Y_{0}^{\mathfrak{k}}\right) \operatorname{Tr}_{\mathrm{s}} \Lambda^{\cdot\left(\mathfrak{p}^{*}\right) \otimes E}\left[\exp \left(-i \rho^{\Lambda^{\cdot}\left(\mathfrak{p}^{*}\right) \otimes E}\left(Y_{0}^{\mathfrak{k}}\right)+\frac{t}{2} C^{\mathfrak{g}, E}\right)\right] \\
& \times \exp \left(-\left|Y_{0}^{\mathfrak{k}}\right|^{2} / 2 t\right) \frac{d Y_{0}^{\mathfrak{k}}}{(2 \pi t)^{m^{\prime} / 2}}=\exp \left(-\frac{t}{48} \operatorname{Tr}^{\mathfrak{k}}\left[C^{\mathfrak{k}, \mathfrak{k}}\right]\right) \\
& \times\left[\widehat{A}\left(T X, \nabla^{T X}\right) \operatorname{Tr}_{\mathrm{s}} S^{\mathfrak{p} * \otimes E}\left[\exp \left(-\frac{R^{S^{\mathfrak{p}^{*}} \otimes E}}{2 i \pi}-\frac{t}{2} C^{\mathfrak{k}, S^{\mathfrak{p}^{*}} \otimes E}+\frac{t}{2} C^{\mathfrak{g}, E}\right)\right]\right]^{\max } .
\end{aligned}
$$

By (8.22), (8.51), and (8.52), we get

$$
C^{\mathfrak{g}, E}-C^{\mathfrak{k}, S^{\mathfrak{p} *} \otimes E}=C^{\mathfrak{g}, H, E}-\frac{1}{8} \operatorname{Tr}^{\mathfrak{p}}\left[C^{\mathfrak{k}, \mathfrak{p}}\right]-2 \sum_{i=m+1}^{m+m^{\prime}} \widehat{c}\left(\left.\operatorname{ad}\left(e_{i}\right)\right|_{\mathfrak{p}}\right) \rho^{E}\left(e_{i}\right) .
$$

Moreover,

$$
\sum_{i=m+1}^{m+m^{\prime}} \widehat{c}\left(\left.\operatorname{ad}\left(e_{i}\right)\right|_{\mathfrak{p}}\right) \rho^{E}\left(e_{i}\right)=\frac{1}{4} \sum_{1 \leqslant i, j \leqslant m} \widehat{c}\left(e_{i}\right) \widehat{c}\left(e_{j}\right) \rho^{E}\left(\left[e_{i}, e_{j}\right]\right) .
$$

As in (8.48), if $A \in \operatorname{End}(\mathfrak{p})$ is antisymmetric, set

$$
\widehat{c}(A)=-\frac{1}{4} \sum_{1 \leqslant i, j \leqslant m}\left\langle A e_{i}, e_{j}\right) \widehat{c}\left(e_{i}\right) \widehat{c}\left(e_{j}\right) .
$$

Then we have the classical identity

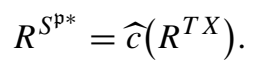


By the first identity in (8.56), and by (8.58)-(8.62), we obtain

$$
\begin{gathered}
\operatorname{Tr}_{\mathrm{s}}{ }^{[1]}\left[\exp \left(-t D^{X, 2} / 2\right)\right]=\left[\widehat { A } ( T X , \nabla ^ { T X } ) \operatorname { T r } _ { \mathrm { s } } { } ^ { S ^ { \mathrm { p } * } \otimes E } \left[\operatorname { e x p } \left(-\widehat{c}\left(\frac{R^{T X}}{2 i \pi}\right)\right.\right.\right. \\
\left.\left.\left.-\frac{R^{E}}{2 i \pi}+\frac{t}{2} C^{\mathfrak{g}, H, E}-\frac{t}{4} \sum_{1 \leqslant i, j \leqslant m} \widehat{c}\left(e_{i}\right) \widehat{c}\left(e_{j}\right) \rho^{E}\left(\left[e_{i}, e_{j}\right]\right)\right)\right]\right]^{\max } .
\end{gathered}
$$

By a computation similar to the one in Theorem 1.3, we get

$$
\begin{aligned}
\left(\nabla^{S^{\mathfrak{p}} \otimes E}+\sqrt{\frac{t}{2}} \sum_{i=1}^{m} \widehat{c}\left(e_{i}\right) \rho^{E}\left(e_{i}\right)\right)^{2}= & \widehat{c}\left(R^{T X}\right)+R^{E}-\frac{t}{2} C^{\mathfrak{g}, H, E} \\
& +\frac{t}{4} \sum_{1 \leqslant i, j \leqslant m} \widehat{c}\left(e_{i}\right) \widehat{c}\left(e_{j}\right) \rho^{E}\left(\left[e_{i}, e_{j}\right]\right) .
\end{aligned}
$$

Let $\varphi$ be the automorphism of $\Lambda^{\text {even }}\left(\mathfrak{p}^{*}\right)$ which maps $\alpha \in \Lambda^{k}\left(\mathfrak{p}^{*}\right)$ to $(2 i \pi)^{-k / 2} \alpha$. By (8.63), (8.64), we get

$$
\begin{aligned}
& \operatorname{Tr}_{\mathrm{s}}{ }^{[1]}\left[\exp \left(-t D^{X, 2} / 2\right)\right] \\
& =\left[\widehat{A}\left(T X, \nabla^{T X}\right) \varphi \operatorname{Tr}_{\mathrm{s}}^{S^{\mathrm{p} *} \otimes E}\left[\exp \left(-\left(\nabla^{S^{\mathrm{p} *} \otimes E}+\sqrt{\frac{t}{2}} \sum_{i=1}^{m} \widehat{c}\left(e_{i}\right) \rho^{E}\left(e_{i}\right)\right)^{2}\right)\right]\right]^{\max }
\end{aligned}
$$

The theory of superconnections guarantees that the cohomology class of the closed form in the right-hand side of (8.65) does not depend on $t$. Because of invariance under the left action of $G$, the forms themselves do not depend on $t$. For $t=0$, we can then proceed as in [11, Theorem 7.8.2], or make a simple computation, and we get the second identity in (8.56). The proof of our theorem is completed.

Let $T$ be a maximal torus in $K$, and let $\mathfrak{t} \subset \mathfrak{k}$ be its Lie algebra. Set

$$
\mathfrak{b}=\{f \in \mathfrak{p},[f, \mathfrak{t}]=0\}
$$

Put

$$
\mathfrak{h}=\mathfrak{b} \oplus \mathfrak{t}
$$

By [32, p. 129], we know that $\mathfrak{h}$ is a Cartan subalgebra of $\mathfrak{g}$, that dim $\mathfrak{t}$ is the complex rank of $K$, and that $\operatorname{dim} \mathfrak{h}$ is the complex rank of $G$. Also, if $m$ is odd, $\mathfrak{b}$ is of odd dimension $\geqslant 1$.

We still define $\operatorname{Tr}_{\mathrm{s}}{ }^{[1]}\left[\left(N^{\Lambda^{*}\left(T^{*} X\right)}-\frac{m}{2}\right) \exp \left(-t D^{X, 2} / 2\right)\right]$ by a formula similar to (8.53). Now, we extend part of [11, Theorem 7.9.1], where the case of a trivial $E$ was considered. 
Theorem 8.6. For any $t>0$, the following identity holds:

$$
\begin{aligned}
& \operatorname{Tr}_{\mathrm{s}}{ }^{[1]}\left[\left(N^{\Lambda^{*}\left(T^{*} X\right)}-\frac{m}{2}\right) \exp \left(-t D^{X, 2} / 2\right)\right]=\frac{1}{(2 \pi t)^{m / 2}} \exp \left(\frac{t}{48} \operatorname{Tr}^{\mathfrak{k}}\left[C^{\mathfrak{k}, \mathfrak{k}}\right]+\frac{t}{16} \operatorname{Tr}^{\mathfrak{p}}\left[C^{\mathfrak{k}, \mathfrak{p}}\right]\right) \\
& \quad \times \int_{\mathfrak{k}} J_{1}\left(Y_{0}^{\mathfrak{k}}\right) \operatorname{Tr}_{\mathrm{s}}{ }^{\Lambda^{\prime}\left(\mathfrak{p}^{*}\right) \otimes E}\left[\left(N^{\Lambda \cdot\left(\mathfrak{p}^{*}\right)}-\frac{m}{2}\right) \exp \left(-i \rho^{\Lambda \cdot\left(\mathfrak{p}^{*}\right) \otimes E}\left(Y_{0}^{\mathfrak{k}}\right)+\frac{t}{2} C^{\mathfrak{g}, E}\right)\right] \\
& \quad \times \exp \left(-\left|Y_{0}^{\mathfrak{k}}\right|^{2} / 2 t\right) \frac{d Y_{0}^{\mathfrak{k}}}{(2 \pi t)^{m^{\prime} / 2}} .
\end{aligned}
$$

If $m$ is even, or if $m$ is odd and $\operatorname{dim} \mathfrak{b} \geqslant 3$, (8.68) vanishes.

Proof. By [11, Theorem 6.1.1] and by Proposition 8.4, we get (8.68). Instead of (8.57), we get

$$
\begin{aligned}
& \operatorname{Tr}_{\mathrm{S}} \Lambda^{\cdot\left(\mathfrak{p}^{*}\right) \otimes E}\left[\left(N^{\Lambda^{*}\left(\mathfrak{p}^{*}\right)}-\frac{m}{2}\right) \exp \left(-i \rho^{\Lambda^{*}\left(\mathfrak{p}^{*}\right) \otimes E}\left(Y_{0}^{\mathfrak{k}}\right)+\frac{t}{2} C^{\mathfrak{g}, E}\right)\right] \\
& \quad=\operatorname{Tr}_{\mathrm{s}} \Lambda^{\cdot\left(\mathfrak{p}^{*}\right)}\left[\left(N^{\Lambda^{\cdot}\left(\mathfrak{p}^{*}\right)}-\frac{m}{2}\right) \exp \left(-i \operatorname{ad}\left(Y_{0}^{\mathfrak{k}}\right)\right)\right] \operatorname{Tr}^{E}\left[\exp \left(-i \rho^{E}\left(Y_{0}^{\mathfrak{k}}\right)+\frac{t}{2} C^{\mathfrak{g}, E}\right)\right] .
\end{aligned}
$$

By proceeding as in $[11$, equations (7.9.1), (7.9.2)], under the given conditions, the first term in the right-hand side of (8.69) vanishes identically. The proof of our theorem is completed.

Remark 8.7. The same arguments as in [11] also show that, under the conditions of the second part of Theorem 8.6, the orbital integrals where 1 is replaced by any semisimple $\gamma \in G$ also vanish. If $\Gamma$ is a torsion-free discrete cocompact subgroup of $G$, and if $Z=$ $\Gamma \backslash X$, this implies the vanishing of the analytic torsion of $Z$ with coefficients in the flat bundle $F$. In that respect, the conditions that were given above are exactly the ones that guarantee the vanishing of the analytic torsion with trivial $F$. As shown in $[11$, Remark 7.9.2], where the connected simple Lie groups $G$ such that $m$ is odd and $\operatorname{dim} \mathfrak{b}=1$ are listed, these are exactly the conditions found by Moscovici and Stanton [40, Corollary 2.2] for the vanishing of the ordinary analytic torsion when $F$ is unitarily flat. Also, note that the vanishing result at the end of our theorem has already been established by Bergeron and Venkatesh in [3, Proposition 5.2].

\subsection{The vanishing of the forms $d_{t}$}

We use the notation of $\S 2$, with $S$ taken to be a point.

By (2.19), (8.15), and (8.36), we get

$$
\sigma\left(\mathcal{A}_{t}^{2}\right)=-\frac{1}{2}\left\langle\theta^{\mathfrak{p}, 2}, \widehat{\theta}^{\mathfrak{p}, 2}\right\rangle-\theta^{\mathfrak{p}, 2}+t\left|\widehat{\theta}^{\mathfrak{p}}\right|^{2}+t \widehat{\theta}^{\mathfrak{p}, 2} .
$$

We take the compact manifold $N$ as in $\S 1.4$, and we define the function $R(A)$ as in (1.23). By (2.55), we get

$$
d_{t}=-\frac{1}{2}(2 i \pi)^{m / 2} \varphi \int^{\widehat{B}} \sqrt{t} \theta^{\mathfrak{p}} \wedge \widehat{\theta}^{\mathfrak{p}} \exp \left(-\sigma\left(\mathcal{A}_{t}^{2}\right)\right) R(0) .
$$


Theorem 8.8. If $m$ is even, or if $m$ is odd, and $\operatorname{dim} \mathfrak{b} \geqslant 3$, then

$$
\int^{\widehat{B}} \theta^{\mathfrak{p}} \wedge \widehat{\theta}^{\mathfrak{p}} \exp \left(\frac{1}{2}\left\langle\theta^{\mathfrak{p}, 2}, \widehat{\theta}^{\mathfrak{p}, 2}\right\rangle+\theta^{\mathfrak{p}, 2}-t \widehat{\theta}^{\mathfrak{p}, 2}\right)=0 .
$$

Under the above conditions, for $t \geqslant 0$,

$$
d_{t}=0
$$

Proof. If $m$ is even, (8.72) is trivial. Let us assume that $m$ is odd, and that $\operatorname{dim} \mathfrak{b} \geqslant 3$. As usual in Chern-Weil theory, we first replace $\frac{1}{2} \theta^{\mathfrak{p}, 2}$ by $A \in \mathfrak{k}$. We will show that

$$
\int^{\widehat{B}} \widehat{\theta}^{\mathfrak{p}} \exp \left(\left\langle A, \widehat{\theta}^{\mathfrak{p}, 2}\right\rangle-t \widehat{\theta}^{\mathfrak{p}, 2}\right)=0,
$$

from which (8.72), (8.73) will follow.

Let $e_{m+1}, \ldots, e_{m+m^{\prime}}$ be an orthonormal basis of $\mathfrak{k}$. If $e \in \mathfrak{k}$, let $\overline{\boldsymbol{e}}$ denote the corresponding constant vector field on $\mathfrak{g}$. Then

$$
\bar{e}=\sum_{i=m+1}^{m+m^{\prime}}\left\langle e, e_{i}\right\rangle \bar{e}_{i}
$$

We will rewrite (8.75) in the form

$$
\bar{e}=\left\langle e, \sum_{i=m+1}^{m+m^{\prime}} e_{i} \otimes \bar{e}_{i}\right\rangle
$$

In particular, in (8.74),

$$
\widehat{\theta}^{\mathfrak{p}, 2}=\left\langle\widehat{\theta}^{\mathfrak{p}, 2}, \sum_{i=m+1}^{m+m^{\prime}} e_{i} \otimes \bar{e}_{i}\right\rangle .
$$

By (8.77), equation (8.74) can be rewritten in the form

$$
\int^{\widehat{B}} \widehat{\theta}^{\mathfrak{p}} \exp \left(\left\langle A-t \sum_{i=m+1}^{m+m^{\prime}} e_{i} \otimes \bar{e}_{i}, \widehat{\theta}^{\mathfrak{p}, 2}\right\rangle\right)=0 .
$$

Since the $\overline{\boldsymbol{e}}_{i}$ lie in a commutative algebra, to prove (8.78), we only need to show that, if $C \in \mathfrak{k}$

$$
\int^{\widehat{B}} \widehat{\theta}^{\mathfrak{p}} \exp \left(\left\langle C, \widehat{\theta}^{\mathfrak{p}, 2}\right\rangle\right)=0 .
$$

Of course we can assume that $C \in \mathfrak{t}$. If $\operatorname{dim} \mathfrak{b} \geqslant 3$, the kernel of $\left.\operatorname{ad}(C)\right|_{\mathfrak{p}}$ is of dimension $\geqslant 3$. Therefore it is clear that the left-hand side of (8.79) vanishes. This completes the proof of our theorem. 


\subsection{The $W$-invariant for $\mathrm{SL}_{2}(\mathbf{C})$}

Now, we assume that $G=\mathrm{SL}_{2}(\mathbf{C})$. Let $\mathfrak{g}=\mathrm{sl}_{2}(\mathbf{C})$ be the Lie algebra of $\mathrm{SL}_{2}(\mathbf{C})$, i.e., the algebra of trace-free $(2,2)$ matrices. Consider the Cartan involution $\Theta: g \rightarrow g^{*-1}$ of $G$. Let $K=\mathrm{SU}(2)$. Then $K$ is the fixed point set of $\Theta$, and a maximal compact subgroup of $G$. Let $\mathfrak{k}=s u(2)$ be the Lie algebra of $K$, i.e., the algebra of skew-adjoint trace-free $(2,2)$ matrices.

Let $\mathfrak{p}$ be the vector space of self-adjoint trace-free $(2,2)$ matrices. Then

$$
\mathfrak{p}=i \mathfrak{k} .
$$

Moreover, we have the Cartan decomposition,

$$
\mathfrak{g}=\mathfrak{p} \oplus \mathfrak{k} .
$$

Clearly, $\mathrm{SL}_{2}(\mathbf{C})$ is the complexification of $\mathrm{SU}(2)$, and $\mathfrak{g}$ is the complexification of $\mathfrak{k}$.

Let $E=\mathbf{C}^{2}$, and let $\rho^{E}: \mathrm{SL}_{2}(\mathbf{C}) \rightarrow \operatorname{Aut}(E)$ be the canonical representation. We equip $\mathfrak{g}$ with the bilinear form

$$
B(a, b)=\frac{1}{2} \operatorname{Re} \operatorname{Tr}[a b] .
$$

Then $B$ is an invariant symmetric bilinear form on $\mathfrak{g}$, the splitting (8.81) is orthogonal with respect to $B$, and $B$ is negative on $\mathfrak{k}$, and positive on $\mathfrak{p}$.

Consider the Pauli matrices,

$$
\mathrm{i}=\left[\begin{array}{cc}
0 & -1 \\
1 & 0
\end{array}\right], \quad \mathrm{j}=\left[\begin{array}{ll}
0 & i \\
i & 0
\end{array}\right], \quad \mathrm{k}=\left[\begin{array}{cc}
-i & 0 \\
0 & i
\end{array}\right] .
$$

Then $\mathrm{i}^{2}=-1, \mathrm{j}^{2}=-1, \mathrm{k}^{2}=-1$, the matrices $\mathrm{i}, \mathrm{j}, \mathrm{k}$ anticommute, and $\mathrm{ij}=\mathrm{k}, \mathrm{ik}=-j$, $\mathrm{jk}=\mathrm{i}$. From the above, we get

$$
[\mathrm{i}, \mathrm{j}]=2 \mathrm{k}, \quad[\mathrm{i}, \mathrm{k}]=-2 \mathrm{j}, \quad[\mathrm{j}, \mathrm{k}]=2 \mathrm{i} .
$$

Also, $\mathrm{i}, \mathrm{j}, \mathrm{k}$ is an orthonormal basis of $\mathfrak{k}$ for the scalar product induced by $-B$. Therefore, $i \mathrm{i}, i \mathrm{j}, i \mathrm{k}$ is an orthonormal basis of $\mathfrak{p}$. We deduce that the induced metric on $X=G / K$ has constant sectional curvature -4 .

We use the notation of $\S 8.2$. By the above, it follows that

$$
\mathrm{i}^{2}+\mathrm{j}^{2}+\mathrm{k}^{2}=\Delta^{\mathfrak{k}}, \quad\left|\widehat{\theta}^{\mathfrak{p}}\right|^{2}=\Delta^{\mathfrak{p}} .
$$

Let $L$ be the differential operator on $\mathfrak{g}$,

$$
L=\mathrm{i}(i \mathrm{i})+\mathrm{j}(i \mathrm{j})+\mathrm{k}(i \mathrm{k}) .
$$

Recall that the algebra $\mathscr{A}^{U}$ was defined in $\S 1.4$. As explained in that subsection, $\Delta^{\mathfrak{p}}$ can be viewed as a nonnegative operator acting on $\mathscr{A}^{U}$. Its fractional powers are then well defined. Similarly $-L^{2}$ can be viewed as a nonnegative operator. Moreover, by the Cauchy-Schwarz inequality,

$$
-L^{2} \leqslant \Delta^{\mathfrak{p}}\left(-\Delta^{\mathfrak{k}}\right)
$$

By (8.87), the action of $\Delta^{\mathfrak{p},-3 / 2} L^{2}$ on $\mathscr{A}^{U}$ is well defined. 
Theorem 8.9. For $t \geqslant 0$, we have the identities of operators acting on $\mathscr{A}^{U}$,

$$
\begin{aligned}
& {\left[\int^{\widehat{B}} \theta^{\mathfrak{p}} \wedge \widehat{\theta}^{\mathfrak{p}} \exp \left(\frac{1}{2}\left\langle\theta^{\mathfrak{p}, 2}, \widehat{\theta}^{\mathfrak{p}, 2}\right\rangle+\theta^{\mathfrak{p}, 2}-t \widehat{\theta}^{\mathfrak{p}, 2}-t\left|\widehat{\theta}^{\mathfrak{p}}\right|^{2}\right)\right]^{\max }} \\
& =\frac{2}{\pi^{3 / 2}}\left(\Delta^{\mathfrak{p}}-2 t L^{2}\right) \exp \left(-t \Delta^{\mathfrak{p}}\right), \\
& {\left[\frac{1}{2} \int_{0}^{+\infty}\left[\int^{\widehat{B}} \sqrt{t} \theta^{\mathfrak{p}} \wedge \widehat{\theta}^{\mathfrak{p}} \exp \left(\frac{1}{2}\left\langle\theta^{\mathfrak{p}, 2}, \widehat{\theta}^{\mathfrak{p}, 2}\right\rangle+\theta^{\mathfrak{p}, 2}-t \widehat{\theta}^{\mathfrak{p}, 2}-t\left|\widehat{\theta}^{\mathfrak{p}}\right|^{2}\right)\right] \frac{d t}{t}\right]^{\max }} \\
& =\frac{1}{\pi}\left(\Delta^{\mathfrak{p}, 1 / 2}-\Delta^{\mathfrak{p},-3 / 2} L^{2}\right) .
\end{aligned}
$$

Proof. Since $\operatorname{dim} \mathfrak{p}=3$, we get

$$
\exp \left(\frac{1}{2}\left\langle\theta^{\mathfrak{p}, 2}, \widehat{\theta}^{\mathfrak{p}, 2}\right\rangle+\theta^{\mathfrak{p}, 2}-t \widehat{\theta}^{\mathfrak{p}, 2}\right)=\left(1+\frac{1}{2}\left\langle\theta^{\mathfrak{p}, 2}, \widehat{\theta}^{\mathfrak{p}, 2}\right\rangle\right)\left(1+\theta^{\mathfrak{p}, 2}\right)\left(1-t \widehat{\theta}^{\mathfrak{p}, 2}\right) .
$$

By (8.89), we obtain

$$
\begin{aligned}
& {\left[\int^{\widehat{B}} \theta^{\mathfrak{p}} \wedge \widehat{\theta}^{\mathfrak{p}} \exp \left(\frac{1}{2}\left\langle\theta^{\mathfrak{p}, 2}, \widehat{\theta}^{\mathfrak{p}, 2}\right\rangle+\theta^{\mathfrak{p}, 2}-t \widehat{\theta}^{\mathfrak{p}, 2}\right)\right]^{\max }} \\
& \quad=\left[\int^{\widehat{B}} \theta^{\mathfrak{p}} \wedge \widehat{\theta}^{\mathfrak{p}}\left(\frac{1}{2}\left\langle\theta^{\mathfrak{p}, 2}, \widehat{\theta}^{\mathfrak{p}, 2}\right\rangle-t \theta^{\mathfrak{p}, 2} \widehat{\theta}^{\mathfrak{p}, 2}\right)\right]^{\max } .
\end{aligned}
$$

Using (1.28) and the commutation relations in (8.84), we get

$$
\begin{aligned}
& {\left[\int^{\widehat{B}} \theta^{\mathfrak{p}} \wedge \widehat{\theta}^{\mathfrak{p}}\left\langle\theta^{\mathfrak{p}, 2}, \widehat{\theta}^{\mathfrak{p}, 2}\right\rangle\right]^{\max }=\frac{4}{\pi^{3 / 2}} \Delta^{\mathfrak{p}},} \\
& {\left[\int^{\widehat{B}} \theta^{\mathfrak{p}} \widehat{\theta}^{\mathfrak{p}} \theta^{\mathfrak{p}, 2} \widehat{\theta}^{\mathfrak{p}, 2}\right]^{\max }=\frac{4}{\pi^{3 / 2}} L^{2} .}
\end{aligned}
$$

By (8.85), (8.90), and (8.91), we get the first identity in (8.88). The second identity follows from the first one. The proof of our theorem is completed.

Remark 8.10. Recall that $\mathfrak{g}$ is itself a complex vector space. Let $\mathcal{H}(\mathfrak{g})$ be the algebra of holomorphic functions on $\mathfrak{g}$. By (8.86), we have the identity of operators acting on $\mathcal{H}(\mathfrak{g}) \cap \mathscr{A}^{U}$,

$$
L=i \Delta^{\mathfrak{k}}=-i \Delta^{\mathfrak{p}} .
$$

From (8.92), we deduce the equality of operators acting on $\mathcal{H}(\mathfrak{g}) \cap \mathscr{A}^{U}$,

$$
\frac{1}{\pi}\left(\Delta^{\mathfrak{p}, 1 / 2}-\Delta^{\mathfrak{p},-3 / 2} L^{2}\right)=\frac{2}{\pi} \Delta^{\mathfrak{p}, 1 / 2}=\frac{2}{\pi}\left(-\Delta^{\mathfrak{k}}\right)^{1 / 2} .
$$

A maximal torus $T=S^{1}$ in $\mathrm{SU}(2)$ is the 1-parameter group $\exp (2 \pi t k), t \in S^{1}=\mathbf{R} / \mathbf{Z}$. Then $k$ is a generator of the Lie algebra of $\mathfrak{t}$, and $2 \pi k$ is a coroot in $T$, so that $\exp (2 \pi k)=1$. Let $P$ be the lattice of weights; i.e., $P$ is the subspace of $t^{*}$ which takes 
integral values on $2 \pi k$. When identifying $\mathfrak{t}$ with $\mathfrak{t}^{*}$ by its scalar product, then $P=\mathbf{Z} / 2 \pi$. A set $P_{++}$of positive weights is just given by $\mathbf{N} / 2 \pi$.

Then $U=\mathrm{SU}(2) \times \mathrm{SU}(2)$ is a compact form of $G=\mathrm{SL}_{2}(\mathbf{C})$, in which $K=\mathrm{SU}(2)$ embeds by the diagonal embedding. Also, $G_{\mathbf{C}}=\mathrm{SL}_{2}(\mathbf{C}) \times \mathrm{SL}_{2}(\mathbf{C})$, and $G$ embeds in $G_{\mathbf{C}}$ by the embedding $g \in \mathrm{SL}_{2}(\mathbf{C}) \rightarrow(g, \Theta g) \in \mathrm{SL}_{2}(\mathbf{C}) \times \mathrm{SL}_{2}(\mathbf{C})$.

By Weyl's unitary trick [32, Proposition 5.7], since $U$ is simply connected, finite-dimensional irreducible representations of $\mathrm{SL}_{2}(\mathbf{C})$ are just the finite-dimensional irreducible representations of $\mathrm{SU}(2) \times \mathrm{SU}(2)$, which are themselves parameterized by two integers $a, b \in \mathbf{N}$ and the corresponding weights $a / 2 \pi, b / 2 \pi$. The corresponding vector space is given by $S^{a} \mathbf{C}^{2} \otimes S^{b} \overline{\mathbf{C}}^{2}$. The holomorphic representations of $\mathrm{SL}_{2}(\mathbf{C})$ correspond to the case where $b=0$, and the antiholomorphic representations to the case $a=0$. If $\rho_{a}$ denotes the representation of $\mathrm{SL}_{2}(\mathbf{C})$ on $S^{a}\left(\mathbf{C}^{2}\right)$, this representation is isomorphic to its dual. The same is true for the representation of $\mathrm{SL}_{2}(\mathbf{C})$ on $S^{b} \overline{\mathbf{C}}^{2}$.

Let $E$ be the hyperplane line bundle on $\mathbf{P}^{1}$. On $\overline{\mathbf{P}}^{1}$, the corresponding hyperplane bundle is denoted $\bar{E}$. For $a \in \mathbf{N}$, the coadjoint orbit $N_{a}$ of $a / 2 \pi \in \mathfrak{t}^{*}$ for $\mathrm{SU}(2)$ can be identified with a point for $a=0$, with $\mathbf{P}^{1}$ for $a>0$. The orbit $N_{a}$ carries a canonical line bundle $L_{a}$. For $a>0$, when identifying $N_{a}$ with $\mathbf{P}^{1}, L_{a}$ is just $E^{a}$.

For $a, b \in \mathbf{N}$, set

$$
N_{a, b}=N_{a} \times \bar{N}_{b} .
$$

Let $q_{1}, q_{2}$ be the obvious projections from $N_{a, b}$ on $N_{a}, \bar{N}_{b}$. For $a, b \in \mathbf{N}$, set

$$
L_{a, b}=q_{1}^{*} L_{a} \otimes q_{2}^{*} \bar{L}_{b} .
$$

Then $\mathrm{SL}_{2}(\mathbf{C})$ acts on $N_{a, b}$ by the map $\left(z, z^{\prime}\right) \rightarrow\left(g z,(\Theta g) z^{\prime}\right)$, and the action of $\mathrm{SL}_{2}(\mathbf{C})$ lifts to $L_{a, b}$. Clearly

$$
H^{(0,0)}\left(N_{a, b}, L_{a, b}\right)=S^{a} \mathbf{C}^{2} \otimes S^{b} \overline{\mathbf{C}}^{2} .
$$

Let $\mu_{a}: N_{a} \rightarrow \operatorname{su}(2)^{*}, \mu_{b}: N_{b} \rightarrow \operatorname{su}(2)^{*}$ be the tautological moment maps. The moment map $\mu_{a, b}$ that is associated with the action of $U$ on $N_{a, b}$ is given by

$$
\mu_{a, b}=\left(q_{1}^{*} \mu_{a}, q_{2}^{*} \bar{\mu}_{b}\right)
$$

Let $\sigma_{a, b, \widehat{\theta}^{\mathfrak{p}}}$ be the section of $\widehat{T X}^{*}$ on $N_{a, b}$ that is defined as in (1.25). We get easily

$$
\sigma_{a, b, \widehat{\theta}^{\mathfrak{p}}}=2 \pi\left\langle q_{1}^{*} \mu_{a}-q_{2}^{*} \bar{\mu}_{b}, i \widehat{\theta}^{\mathfrak{p}}\right\rangle .
$$

By (8.98), $\widehat{\theta}^{\mathfrak{p}}$ is nondegenerate if and only if $a \neq b$.

We equip the line bundle $E$ with its canonical metric. Let $\mathbf{m}: \mathbf{P}^{1} \rightarrow \mathfrak{k}^{*}$ be the corresponding moment map. When identifying $N_{1}$ with $\mathbf{P}^{1}, L_{1}$ maps to $E$, and $\mu_{1}$ to $\mathbf{m}$. Set

$$
\eta=c_{1}\left(E, g^{E}\right)
$$

For $A \in \mathfrak{g}$, set

$$
S(A)=\int_{\mathbf{P}^{1}} \exp (2 i \pi\langle\mathbf{m}, A\rangle+\eta)
$$


Note that $S$ is an even function. When $A \in \operatorname{su}(2), S(A)$ can be calculated using the localization formulae of $[5,29,30]$. If $A \in \mathfrak{t}$, we can write $A$ in the form $A=t k, t \in \mathbf{R}$. Then

$$
S(A)=\frac{\sin (t)}{t} .
$$

For $A \in \mathrm{sl}_{2}(\mathbf{C})$, let $R_{a, b}(A)$ be the function defined in (1.23) that is associated with the previous geometric data. One finds easily that

$$
\begin{aligned}
R_{a, b}(A)= & a S(a A) b S(b \Theta A) \quad \text { if } a>0, b>0, \\
& a S(a A) \quad \text { if } a>0, b=0 .
\end{aligned}
$$

If $b=0$, the function $R_{a, b}$ is holomorphic.

If $a \neq b$, let $W_{a, b}$ be the associated form on $X$.

Theorem 8.11. For $a \neq b$,

$$
W_{a, b}^{\max }=\frac{1}{\pi}\left(\Delta^{\mathfrak{p}, 1 / 2}-\Delta^{\mathfrak{p},-3 / 2} L^{2}\right) R_{a, b}(0) .
$$

For $a>0$,

$$
W_{a, 0}^{\max }=\frac{2}{\pi} a^{2}
$$

For $a>0, b>0$,

$$
\begin{aligned}
W_{a, b}^{\max } & =\frac{2}{3 \pi}\left(3 a^{2} b-b^{3}\right) \quad \text { if } a>b, \\
& =\frac{2}{3 \pi}\left(3 a b^{2}-a^{3}\right) \quad \text { if } b>a .
\end{aligned}
$$

Proof. By (2.75), (8.71), and (8.88), we get (8.103). For $a \in \mathbf{N}, S(a A)$ is an eigenfunction of $-\Delta^{\mathfrak{k}}$ with eigenvalue $a^{2}$. Moreover,

$$
S(0)=1 .
$$

By (8.93), (8.102), (8.103), and (8.106), we get (8.104). Assume now that $a, b \in \mathbf{N}^{*}, a \neq b$. Since $S(a A)$ is a holomorphic function on $\mathfrak{g}$, and $S(b \Theta A)$ is antiholomorphic, we get

$$
L R_{a, b}=i\left(b^{2}-a^{2}\right) R_{a, b} .
$$

Let $f(A), g(A)$ denote functions on $\mathfrak{g}$ that are respectively holomorphic and antiholomorphic. Then

$$
\Delta^{\mathfrak{p}}(f g)=-\left(\Delta^{\mathfrak{k}} f\right) g-f \Delta^{\mathfrak{k}} g+2\left\langle\nabla^{\mathfrak{k}} f, \nabla^{\mathfrak{k}} g\right\rangle .
$$

From (8.108), we get

$$
\Delta^{\mathfrak{p}}(f g)=\Delta^{\mathfrak{k}}(f g)-2\left(\Delta^{\mathfrak{k}} f\right) g-2 f \Delta^{\mathfrak{k}} g .
$$

Using (8.109), we obtain

$$
\Delta^{\mathfrak{p}} R_{a, b}=\left(\Delta^{\mathfrak{k}}+2\left(a^{2}+b^{2}\right)\right) R_{a, b}
$$


By (8.107), (8.110), we obtain

$$
\begin{aligned}
& \left(\Delta^{\mathfrak{p}}-2 t L^{2}\right) \exp \left(-t \Delta^{\mathfrak{p}}\right) R_{a, b} \\
& \quad=\left(-\frac{\partial}{\partial t}+2 t\left(b^{2}-a^{2}\right)^{2}\right) \exp \left(-t\left(\Delta^{\mathfrak{k}}+2\left(a^{2}+b^{2}\right)\right)\right) R_{a, b} .
\end{aligned}
$$

Since the function $R_{a, b}(A), A \in \mathfrak{k}$ is invariant by rotations, for $t>0$, we get

$$
\exp \left(t \Delta^{\mathfrak{k}}\right) R_{a, b}(0)=\frac{4 \pi}{(4 \pi t)^{3 / 2}} \int_{0}^{+\infty} \exp \left(-r^{2} / 4 t\right) R_{a, b}(r) r^{2} d r
$$

Moreover,

$$
\sin (a r) \sin (b r)=\frac{1}{2}(\cos ((a-b) r)-\cos ((a+b) r)) .
$$

By (8.101), (8.102), (8.112), and (8.113), we obtain

$$
\exp \left(t \Delta^{\mathfrak{k}}\right) R_{a, b}(0)=\frac{1}{4 t}\left(e^{-(a-b)^{2} t}-e^{-(a+b)^{2} t}\right) .
$$

Also, (8.114) is analytic in the variable $t \in \mathbf{C}$. By (8.114), for $t>0$, we get

$$
\exp \left(-t \Delta^{\mathfrak{k}}\right) R_{a, b}(0)=\frac{1}{4 t}\left(e^{(a+b)^{2} t}-e^{(a-b)^{2} t}\right) .
$$

By (8.111), (8.115), we get

$$
\begin{aligned}
& \left(\Delta^{\mathfrak{p}}-2 t L^{2}\right) \exp \left(-t \Delta^{\mathfrak{p}}\right) R_{a, b}(0) \\
& \quad=\left(-\frac{\partial}{\partial t}+2\left(b^{2}-a^{2}\right)^{2} t\right) \frac{1}{4 t}\left(e^{-(a-b)^{2} t}-e^{(a+b)^{2} t}\right) .
\end{aligned}
$$

By (8.88), (8.103), we get

$$
W_{a, b}^{\max }=\pi^{-3 / 2} \int_{0}^{+\infty} t^{-1 / 2}\left(-\frac{\partial}{\partial t}+2\left(b^{2}-a^{2}\right)^{2} t\right) \frac{1}{4 t}\left(e^{-(a-b)^{2} t}-e^{(a+b)^{2} t}\right) d t .
$$

Equivalently,

$$
\begin{aligned}
W_{a, b}^{\max }= & -\frac{1}{8} \pi^{-3 / 2} \Gamma(-3 / 2)\left(|a-b|^{3}-|a+b|^{3}\right) \\
& +\frac{1}{2 \pi^{3 / 2}} \Gamma(1 / 2)\left(b^{2}-a^{2}\right)^{2}\left(|a-b|^{-1}-|a+b|^{-1}\right) .
\end{aligned}
$$

Also, we have the identities

$$
\Gamma(1 / 2)=\sqrt{\pi}, \quad \Gamma(-3 / 2)=\frac{4}{3} \sqrt{\pi} .
$$

By (8.118), (8.119), we get (8.105). The proof of our theorem is completed.

Take $a, b \in \mathbf{N}$ with $a \neq b$. Let $\Gamma$ be a torsion-free discrete cocompact subgroup of $G=$ $\mathrm{SL}_{2}(\mathbf{C})$. Then $Z=\Gamma \backslash X$ is a compact manifold. For $p \in \mathbf{N}, F_{p}=S^{p a} \mathbf{C}^{2} \otimes S^{p b} \overline{\mathbf{C}}^{2}$ descends to a flat Hermitian vector bundle on $Z$, which we also denote $F_{p}$. Let $T_{h}\left(Z, \nabla F_{p}, g^{F_{p}}\right) \in \mathbf{R}$ denote the corresponding analytic torsion. Since $Z$ is odd dimensional, for $p \in \mathbf{N}$ large 
enough, as the notation indicates, $T_{h}\left(Z, \nabla^{F_{p}}, g^{F_{p}}\right)$ does not depend on the metric on $T Z$. By Theorem 7.1 , for $a>0, b>0, a \neq b$, as $p \rightarrow+\infty$,

$$
p^{-3} \mathcal{T}_{h}\left(Z, \nabla^{F_{p}}, g^{F_{p}}\right)=W_{a, b}^{\max } \operatorname{Vol}(Z)+\mathcal{O}\left(p^{-1}\right) .
$$

If $a>0, b=0$, instead of (8.120), we get

$$
p^{-2} \mathcal{T}_{h}\left(Z, \nabla^{F_{p}}, g^{F_{p}}\right)=W_{a, 0}^{\max } \operatorname{Vol}(Z)+\mathcal{O}\left(p^{-1}\right) .
$$

Equation (8.121) is the main result obtained by Müller in [43, Theorem 1.1]. In [43], the curvature of $X=G / K$ is -1 instead of -4 , which explains the difference in the evaluation of the right-hand side in (8.121).

\subsection{The nondegenerate representations of $U$}

We consider again the case of a general reductive group $G$. We assume that $m$ is odd, and that $\operatorname{dim} \mathfrak{b}=1$. The connected simple Lie groups for which this condition holds were described by Moscovici and Stanton [40, Corollary 2.2], [11, Remark 7.9.2].

Set

$$
\mathfrak{t}_{U}=i \mathfrak{b} \oplus \mathfrak{t}
$$

Then $\mathfrak{t}_{U}$ is the Lie algebra of a maximal torus $T_{U} \subset U$. Moreover, $\Theta$ acts on $\mathfrak{t}_{U}$ and preserves the splitting in (8.122). This action lifts to the natural action of $\Theta$ on $T_{U} \subset U$. Let $W_{U}$ be the Weyl group of $U$ associated with $T_{U}$.

Take $\lambda \in \mathfrak{t}_{U}^{*}$, and let $N_{\lambda}$ be the coadjoint orbit of $\lambda$ in $\mathfrak{u}^{*}$. Then

$$
N_{\lambda} \cap \mathfrak{t}_{U}^{*}=W_{U} \lambda
$$

Moreover, if $U_{\lambda} \subset U$ denotes the stabilizer of $\lambda$, then

$$
N_{\lambda}=U / U_{\lambda}
$$

Then $N_{\lambda}$ is a compact Kähler $U$-manifold. It is equipped with a closed symplectic (1,1)-form $\eta_{\lambda}$, and $v \in N_{\lambda} \rightarrow v \in \mathfrak{u}^{*}$ is a moment map for the action of $U$ on $N_{\lambda}$. The associated function $R_{\lambda}(A), A \in \mathfrak{u}$ in (1.23) is given by

$$
R_{\lambda}(A)=\int_{N_{\lambda}} \exp \left(2 i \pi\langle v, A\rangle+\eta_{\lambda}\right) .
$$

The function $R_{\lambda}$ can be computed using the formulae of $[5,29,30]$. Also, $R_{\lambda}$ is an eigenfunction of $-\Delta^{\mathfrak{u}}$ with eigenvalue $4 \pi^{2}|\lambda|^{2}$.

Proposition 8.12. The form $\widehat{\theta}^{\mathfrak{p}}$ is nondegenerate with respect to $N_{\lambda}$ if and only if $W_{U} \lambda \cap$ $\mathfrak{t}^{*}=\varnothing$.

Proof. Observe that $\widehat{\theta}^{\mathfrak{p}}$ is degenerate if and only if there is $v \in N_{\lambda}$ such that $v$ is orthogonal to $i \mathfrak{p}$, i.e., $v \in \mathfrak{k}^{*}$. By making $K$ act on $v$, we may as well assume that $v \in \mathfrak{t}^{*}$. Therefore, there exists $w \in W_{U}$ such that $w \lambda=v$. The proof of our proposition is completed.

Remark 8.13. One verifies easily that, if $G=\mathrm{SL}_{2}(\mathbf{C})$, the condition in Proposition 8.12 is exactly the one stated after equation (8.98). 


\subsection{The asymptotics as $p \rightarrow+\infty$ of the orbital integrals}

We still assume that $m$ is odd, and that $\operatorname{dim} \mathfrak{b}=1$. Let $R_{U,+} \subset \mathfrak{t}_{U}^{*}$ be a positive root system for $U$. Let $\mathcal{C} \subset \mathfrak{t}_{U}$ be the positive Weyl chamber, and let $P_{++} \subset \mathfrak{t}_{U}^{*}$ denote the set of positive weights.

If $\lambda \in P_{++}$, let $\rho_{\lambda}$ be the irreducible unitary representation of $U$ of highest weight $\lambda$ on the vector space $E_{\lambda}$. We still denote by $\rho_{\lambda}$ the corresponding representation of $G$ or $G_{\mathbf{C}}$.

Here, we take $N=N_{\lambda}$. Then $N_{\lambda}$ is a compact Kähler manifold of complex dimension $n$. Moreover, $N_{\lambda}$ is equipped with the canonical holomorphic Hermitian line bundle $L$, and, for $p \in \mathbf{N}$,

$$
E_{p \lambda}=H^{(0,0)}\left(N, L^{p}\right) .
$$

Let $F_{p}$ denote the associated flat Hermitian bundle on $X$ that is associated with $E_{p \lambda}$. Let $D_{p}^{X}$ denote the Dirac operator acting on $\Omega^{\cdot}\left(X, F_{p}\right)$ that was defined in (4.7). Recall that, for $t \geqslant 0$, the form $e_{t}$ on $X$ was defined in Definition 2.9.

Theorem 8.14. For $t>0$, as $p \rightarrow+\infty$,

$$
\begin{aligned}
& p^{-n-1} \operatorname{Tr}_{\mathrm{s}}{ }^{[1]}\left[\left(\frac{N^{\Lambda^{*}\left(T^{*} X\right)}}{2}-\frac{m}{4}\right) \exp \left(-t D_{p}^{X, 2} / 2 p^{2}\right)\right]=\left[e_{t / 2}\right]^{\max }+\mathcal{O}\left(p^{-1}\right), \\
& p^{-n-1} \operatorname{Tr}_{\mathrm{s}}{ }^{[1]}\left[\left(\frac{N^{\Lambda^{*}\left(T^{*} X\right)}}{2}-\frac{m}{4}\right)\left(1-t D_{p}^{X, 2} / p^{2}\right) \exp \left(-t D_{p}^{X, 2} / 2 p^{2}\right)\right] \\
& =\left[d_{t / 2}\right]^{\max }+\mathcal{O}\left(p^{-1}\right) .
\end{aligned}
$$

Proof. By (8.68), (8.69), for $p \in \mathbf{N}^{*}$, we get

$$
\begin{aligned}
\operatorname{Tr}_{\mathrm{s}}{ }^{[1]} & {\left[\left(N^{\Lambda^{*}\left(T^{*} X\right)}-\frac{m}{2}\right) \exp \left(-t D_{p}^{X, 2} / 2 p^{2}\right)\right] } \\
= & \frac{p^{m}}{(2 \pi t)^{m / 2}} \exp \left(\frac{t}{48 p^{2}} \operatorname{Tr}^{\mathfrak{k}}\left[C^{\mathfrak{k}, \mathfrak{k}}\right]+\frac{t}{16 p^{2}} \operatorname{Tr}^{\mathfrak{p}}\left[C^{\mathfrak{k}, \mathfrak{p}}\right]\right) \\
& \times \int_{\mathfrak{k}} J_{1}\left(Y_{0}^{\mathfrak{k}} / p\right) \operatorname{Tr}_{\mathrm{s}}{ }^{\Lambda^{\prime}\left(\mathfrak{p}^{*}\right)}\left[\left(N^{\Lambda^{*}\left(\mathfrak{p}^{*}\right)}-\frac{m}{2}\right) \exp \left(-i \operatorname{ad}\left(Y_{0}^{\mathfrak{k}}\right) / p\right)\right] \\
& \times \operatorname{Tr}^{E_{p \lambda}}\left[\exp \left(-i \rho^{E_{p \lambda}}\left(Y_{0}^{\mathfrak{k}}\right) / p+\frac{t}{2 p^{2}} C^{\mathfrak{g}, E_{p \lambda}}\right)\right] \exp \left(-\left|Y_{0}^{\mathfrak{k}}\right|^{2} / 2 t\right) \frac{d Y_{0}^{\mathfrak{k}}}{(2 \pi t)^{m^{\prime} / 2}} .
\end{aligned}
$$

Let $C^{\mathfrak{u}}$ denote the Casimir operator for the Lie algebra $\mathfrak{u}$ equipped with its canonical scalar product, and let $C^{\mathfrak{u}, E_{p \lambda}}$ denote its action on $E_{p \lambda}$. Clearly,

$$
C^{\mathfrak{g}}=-C^{\mathfrak{u}} \quad \text { in } U \mathfrak{g}_{\mathbf{C}} .
$$

Let $\rho_{U}$ be the half sum of the positive roots of $U$. Classically,

$$
C^{\mathfrak{u}, E_{p \lambda}}=4 \pi^{2}\left(\left|p \lambda+\rho_{U}\right|^{2}-\left|\rho_{U}\right|^{2}\right) .
$$

By $(8.129),(8.130)$, as $p \rightarrow+\infty$,

$$
\frac{C^{\mathfrak{g}, E_{p \lambda}}}{p^{2}} \rightarrow-4 \pi^{2}|\lambda|^{2}
$$


Also, by equation (3.35) in Theorem 3.6, as $p \rightarrow+\infty$,

$$
p^{-n} \operatorname{Tr}^{E_{p \lambda}}\left[\exp \left(-i \rho^{E_{p \lambda}}\left(Y_{0}^{\mathfrak{k}}\right) / p\right)\right]=R_{\lambda}\left(-i Y_{0}^{\mathfrak{k}}\right)+\mathcal{O}\left(p^{-1}\right) .
$$

Let $\mathfrak{b}^{\perp} \subset \mathfrak{p}$ be the space orthogonal to the one-dimensional line $\mathfrak{b}$. Take $Y_{0}^{\mathfrak{k}} \in \mathfrak{t}$. Since $m$ is odd, and $\operatorname{ad}\left(Y_{0}^{\mathfrak{k}}\right)$ vanishes on $\mathfrak{b}$, by the elementary [11, equation (7.9.1)], we get

$$
\operatorname{Tr}_{\mathrm{s}} \Lambda^{\cdot\left(\mathfrak{p}^{*}\right)}\left[\left(N^{\Lambda^{*}\left(\mathfrak{p}^{*}\right)}-\frac{m}{2}\right) \exp \left(-i \operatorname{ad}\left(Y_{0}^{\mathfrak{k}}\right) / p\right)\right]=-\operatorname{det}^{\mathfrak{b}^{\perp}}\left[1-\exp \left(i \operatorname{ad}\left(Y_{0}^{\mathfrak{k}}\right) / p\right)\right] .
$$

By (8.133), since $\operatorname{dim} \mathfrak{b}^{\perp}$ is even, as $p \rightarrow+\infty$,

$$
\begin{aligned}
& p^{(m-1)} \operatorname{Tr}_{\mathrm{s}}{ }^{\Lambda^{\cdot}\left(\mathfrak{p}^{*}\right)}\left[\left(N^{\Lambda^{\cdot}\left(\mathfrak{p}^{*}\right)}-\frac{m}{2}\right) \exp \left(-i \operatorname{ad}\left(Y_{0}^{\mathfrak{k}}\right) / p\right)\right] \\
& =-\operatorname{det}\left[\left.i \operatorname{ad}\left(Y_{0}^{\mathfrak{k}}\right)\right|_{\mathfrak{b}^{\perp}}\right]+\mathcal{O}\left(p^{-1}\right) .
\end{aligned}
$$

By $(1.29),(8.4)$, the 2 -form $\omega_{\left.\operatorname{ad}\left(Y_{0}^{\mathfrak{k}}\right)\right|_{\mathfrak{p}}}$ on $\mathfrak{p}$ that is associated with $\left.\operatorname{ad}\left(Y_{0}^{\mathfrak{k}}\right)\right|_{\mathfrak{p}}$ is given by

$$
\omega_{\left.\operatorname{ad}\left(Y_{0}^{\mathfrak{k}}\right)\right|_{\mathfrak{p}}}=-\left\langle Y_{0}^{\mathfrak{k}}, \Theta^{\mathfrak{k}}\right\rangle .
$$

Let $\left|\Theta^{\mathfrak{p}}\right|^{2} \in \Lambda^{4}\left(\mathfrak{p}^{*}\right)$ be obtained from $\Theta^{\mathfrak{k}} \in \Lambda^{2}\left(\mathfrak{p}^{*}\right) \otimes \mathfrak{k}$ in (8.4) by taking the square of its norm in $\mathfrak{k}$. By an easy computation in [11, equation (7.5.19)],

$$
\left|\Theta^{\mathfrak{p}}\right|^{2}=0 \text {. }
$$

Of course, this also implies that

$$
\left|\widehat{\Theta}^{p}\right|^{2}=0
$$

If $Y_{0}^{\mathfrak{k}} \in \mathfrak{t}$,

$$
\operatorname{det}\left[\left.i \operatorname{ad}\left(Y_{0}^{\mathfrak{k}}\right)\right|_{\mathfrak{b}^{\perp}}\right]=\operatorname{Pf}\left[\left.i \operatorname{ad}\left(Y_{0}^{\mathfrak{k}}\right)\right|_{\mathfrak{b}^{\perp}}\right]^{2}
$$

As in (2.54), set

$$
L=\sum_{i=1}^{m} e^{i} \wedge \widehat{e}^{i}
$$

By (1.28), (1.31), (8.135), and (8.138), since $m$ is odd, we get

$$
\pi^{-m / 2} \operatorname{det}\left[\left.i \operatorname{ad}\left(Y_{0}^{\mathfrak{k}}\right)\right|_{\mathfrak{b}^{\perp}}\right]=-\left[\int^{\widehat{B}} L \exp \left(\left\langle Y_{0}^{\mathfrak{k}}, \Theta^{\mathfrak{k}}+\widehat{\Theta}^{\mathfrak{k}}\right\rangle\right)\right]^{\max } .
$$

By (8.134), (8.140), for $Y_{0}^{\mathfrak{k}} \in \mathfrak{t}$,

$$
\begin{gathered}
\pi^{-m / 2} p^{(m-1)} \operatorname{Tr}_{\mathrm{s}}{ }^{\Lambda^{*}\left(\mathfrak{p}^{*}\right)}\left[\left(N^{\Lambda^{*}\left(\mathfrak{p}^{*}\right)}-\frac{m}{2}\right) \exp \left(-i \operatorname{ad}\left(Y_{0}^{\mathfrak{k}}\right) / p\right)\right] \\
\rightarrow\left[\int^{\widehat{B}} L \exp \left(\left\langle Y_{0}^{\mathfrak{k}}, \Theta^{\mathfrak{k}}+\widehat{\Theta}^{\mathfrak{k}}\right\rangle\right)\right]^{\max } .
\end{gathered}
$$

Of course, equation (8.141) extends to $Y_{0}^{\mathfrak{k}} \in \mathfrak{k}$. 
By (8.128), (8.131), (8.132), and (8.141), as $p \rightarrow+\infty$,

$$
\begin{aligned}
& p^{-n-1} \operatorname{Tr}_{\mathrm{s}}{ }^{[1]}\left[\left(N^{\Lambda^{*}\left(T^{*} X\right)}-\frac{m}{2}\right) \exp \left(-t D_{p}^{X, 2} / 2 p^{2}\right)\right] \\
& =\frac{\exp \left(-2 \pi^{2} t|\lambda|^{2}\right)}{(2 t)^{m / 2}} \int_{\mathfrak{k}}\left[\int^{\widehat{B}} L \exp \left(\left\langle Y_{0}^{\mathfrak{k}}, \Theta^{\mathfrak{k}}+\widehat{\Theta}^{\mathfrak{k}}\right\rangle\right)\right]^{\max } \\
& \times R_{\lambda}\left(-i Y_{0}^{\mathfrak{k}}\right) \exp \left(-\left|Y_{0}^{\mathfrak{k}}\right|^{2} / 2 t\right) \frac{d Y_{0}^{\mathfrak{k}}}{(2 \pi t)^{m^{\prime} / 2}}+\mathcal{O}\left(p^{-1}\right) .
\end{aligned}
$$

By (8.136), (8.137), if $C$ is the leading term in the right-hand side of (8.142), then

$$
\begin{aligned}
C= & {\left[\int^{\widehat{B}} \frac{L}{\sqrt{2 t}} \exp \left(\frac{1}{2}\left\langle\Theta^{\mathfrak{k}}, \widehat{\Theta}^{\mathfrak{k}}\right\rangle\right)\right.} \\
& \left.\times \exp \left(-2 \pi^{2} t|\lambda|^{2}+\frac{t}{2} \Delta^{\mathfrak{k}}\right) R_{\lambda}(-i A)\right]^{\max }\left(\Theta^{\mathfrak{k}}+\frac{t}{2} \widehat{\Theta}^{\mathfrak{k}}\right) .
\end{aligned}
$$

Since $R(A)$ is a holomorphic function of $A$, by (8.143), we get

$$
\begin{aligned}
C= & {\left[\int^{\widehat{B}} \frac{L}{\sqrt{2 t}} \exp \left(\frac{1}{2}\left\langle\Theta^{\mathfrak{k}}, \widehat{\Theta}^{\mathfrak{k}}\right\rangle-\Theta^{\mathfrak{k}}+\frac{t}{2} \widehat{\Theta}^{\mathfrak{k}}\right)\right.} \\
& \left.\times \exp \left(-2 \pi^{2} t|\lambda|^{2}-\frac{t}{2} \Delta^{\mathfrak{k}}\right) R_{\lambda}\right]^{\max }(0) .
\end{aligned}
$$

Let $\Delta^{\mathfrak{u}}, \Delta^{\mathfrak{k}}, \Delta^{i \mathfrak{p}}$ denote the Laplacians of the Euclidean vector spaces $\mathfrak{u}, \mathfrak{k}, i \mathfrak{p}$. Then

$$
\Delta^{\mathfrak{u}}=\Delta^{\mathfrak{k}}+\Delta^{i \mathfrak{p}} \text {. }
$$

Since

$$
\Delta^{\mathfrak{u}} R_{\lambda}=-4 \pi^{2}|\lambda|^{2} R_{\lambda}
$$

we deduce from (8.145) that

$$
\left(4 \pi^{2}|\lambda|^{2}+\Delta^{\mathfrak{k}}\right) R_{\lambda}=-\Delta^{i \mathfrak{p}} R_{\lambda} .
$$

Since $R$ is analytic,

$$
\Delta^{i \mathfrak{p}} R_{\lambda}=-\Delta^{\mathfrak{p}} R_{\lambda}
$$

By (8.147), (8.148), we get

$$
\exp \left(-2 \pi^{2} t|\lambda|^{2}-\frac{t}{2} \Delta^{\mathfrak{k}}\right) R_{\lambda}=\exp \left(-\frac{t}{2} \Delta^{\mathfrak{p}}\right) R_{\lambda} .
$$

As in (8.85), we get

$$
\Delta^{\mathfrak{p}}=\left|\widehat{\theta}^{\mathfrak{p}}\right|^{2}
$$

By (8.149)-(8.150), we get

$$
\exp \left(-2 \pi^{2} t|\lambda|^{2}-\frac{t}{2} \Delta^{\mathfrak{k}}\right) R_{\lambda}=\exp \left(-\frac{t}{2}\left|\widehat{\theta}^{\mathfrak{p}}\right|^{2}\right) R_{\lambda} .
$$


By (8.3),

$$
\Theta^{\mathfrak{k}}=-\theta^{\mathfrak{p}, 2}, \quad \widehat{\Theta}^{\mathfrak{k}}=-\widehat{\theta}^{\mathfrak{p}, 2} .
$$

By (8.144), (8.151), and (8.152), we obtain

$$
C=\left[\int^{\widehat{B}} \frac{L}{\sqrt{2 t}} \exp \left(\frac{1}{2}\left\langle\theta^{\mathfrak{p}, 2}, \widehat{\theta}^{\mathfrak{p}, 2}\right\rangle+\theta^{\mathfrak{p}, 2}-\frac{t}{2} \widehat{\theta}^{\mathfrak{p}, 2}-\frac{t}{2}\left|\widehat{\theta}^{\mathfrak{p}}\right|^{2}\right) R_{\lambda}\right]^{\max } \text { (0). }
$$

By (2.55), (8.70), we can rewrite (8.153) in the form

$$
C=2\left[e_{t / 2}\right]^{\max } \text {. }
$$

By combining (8.142) and (8.154), we get the first equation in (8.127). Applying the operator $1+2 t \frac{\partial}{\partial t}$ to both sides of this first identity and using equation (2.58) in Theorem 2.10, we get the second identity. The proof of our theorem is completed.

Remark 8.15. The second identity in (8.127) can also be viewed as a consequence of the analogue of Theorem 6.5 in the case of universal covers described in $\S 6.6$. In the present context, when $\lambda$ is such that $\widehat{\theta}^{\mathfrak{p}}$ is nondegenerate, Theorem 7.7 is a consequence of Theorem 8.14, and of the uniform decay estimates as $t \rightarrow+\infty$ that were established in the proof of Theorems 7.1 and 7.7. These estimates are not obvious if one starts from (8.128), because the presence of $J_{1}\left(Y_{0}^{\mathfrak{k}} / p\right)$ in the right-hand side of (8.128) does not allow us to replace $Y_{0}^{\mathfrak{k}}$ by $i Y_{0}^{\mathfrak{k}}$. This point can be seen from another point of view in equations (8.144)-(8.151), where the analyticity of $R_{\lambda}$ is used when $J_{1}\left(Y_{0}^{\mathfrak{k}}\right)$ has disappeared. It is somewhat paradoxical that, from the Weitzenböck equation (8.39), we get a better proof of the required estimates than by the evaluation of the orbital integral in (8.128).

If $\gamma \in G$ is semisimple and nonelliptic, if $\operatorname{Tr}_{\mathrm{s}}{ }^{[\gamma]}$ denotes the corresponding orbital integral, by [11, Theorem 6.1.1], there exist $C>0, c>0$ such that, for $t \in] 0,1], p \in \mathbf{N}^{*}$,

$$
\left|\operatorname{Tr}_{\mathrm{s}}^{[\gamma]}\left[\left(\frac{N^{\Lambda^{*}\left(T^{*} X\right)}}{2}-\frac{m}{4}\right) \exp \left(-t D_{p}^{X, 2} / 2 p^{2}\right)\right]\right| \leqslant C \exp \left(-c p^{2} / t\right) .
$$

Let $\Gamma \subset G$ be a torsion-free discrete cocompact subgroup, let $Z=\Gamma \backslash X$, and assume that $\widehat{\theta}^{\mathfrak{p}}$ is nondegenerate. By Theorems 7.1 and $7.7, \mathcal{T}_{h}\left(g^{T Z}, \nabla^{F_{p}}, g^{F_{p}}\right)$ and $\mathcal{T}_{h}^{\Gamma}\left(g^{T Z}, \nabla^{F_{p}}, g^{F_{p}}\right)$ have the same asymptotics, up to the factor $\operatorname{Vol}(Z)$. Equations $(8.127)$ and (8.155) explain why this is the case. Equation (8.155) is of no use for large $t$. However, by Remark 4.5, for $p \in \mathbf{N}$ large enough, and $t \geqslant 1$, one obtains an extra uniform factor $\exp \left(-c^{\prime} t\right)$ with $c^{\prime}>0$, from which the Selberg trace formula can be properly controlled, so as to explain the identity of the two asymptotics above in this very special case.

By Theorem 4.4, the condition of nondegeneracy of $\widehat{\theta}^{\mathfrak{p}}$ given in Proposition 8.12 implies that, for $p \in \mathbf{N}$ large enough, if $Z=\Gamma \backslash X$ is a compact quotient of $X$, then $H^{\cdot}\left(Z, F_{p}\right)=0$. The same vanishing condition also appears in a different context in Borel and Wallach's work [24, Proposition 2.6.12].

\section{The case of general Lie groups}

The purpose of this section is to extend the results of the previous sections to the case where the underlying Lie group $G$ is arbitrary. In this case, choosing a metric on the line 
bundle $L$ is a fibrewise infinite-dimensional problem. The asymptotics of the analytic torsion cannot be formally reduced to a problem of analysis on the group $G$ as in the previous sections. Of course, the results of this section are more general than the ones that were obtained before.

On the differential geometric side, the construction of the form $\pi_{*} W$-invariant now uses explicitly the properties of the Poisson algebra of the fibres $N$ over $M$. The asymptotics of the analytic torsion forms are obtained by a much more detailed analysis of corresponding Toeplitz operators that act along the fibres $N$. Contrary to what happened in the previous sections, the problem cannot be reduced to a finite-dimensional Lie algebra of such operators. The asymptotics ultimately use the degeneration of the algebra of fibrewise Toeplitz operators to the Poisson algebra, in the same way as, in the previous sections, the bundle of Lie groups $G_{r}$ was degenerating to the bundle of Lie algebras $\mathfrak{g}_{r}$.

This section is organized as follows. In $\S 9.1$, we introduce the geometric setting, which includes in particular the choice of a Hermitian metric $g^{L}$ on the line bundle $L$ on $\mathcal{N}$.

In $\S 9.2$, we elaborate on the differential geometry of the projection $q: \mathcal{N} \rightarrow M$, by considerations very similar to the ones we made in $\S \S 2.1$ and 2.2 on the projection $\pi: M \rightarrow S$.

In $\S 9.3$, we give various properties on the bundle of Poisson algebras $\mathcal{A}$ over $M$. In particular, we construct a connection $\nabla^{\mathcal{A}, u}$ on the bundle $\mathcal{A}$, and we calculate its curvature.

In $\S 9.4$, we extend the construction of the forms $a_{t}, b_{t}$ in $\S 1.12$ to this more general setting. Properties of the Poisson algebra are used here instead of the properties of the Lie algebra $\mathfrak{g}$ in $\S 1.12$.

In $\S 9.5$, we extend the construction of the forms $c_{t}, d_{t}$ in $\S 2.5$.

In $\S 9.6$, which extends $\S 2.6$, we construct the $W$-invariant under a natural assumption of nondegeneracy.

In $\S 9.7$, the unitary connection $\nabla^{F, u}$ is explicitly calculated, and its action on a family of Toeplitz operators is obtained.

In $\S 9.8$, we recall various properties of the algebra of Toeplitz operators in the sense of Ma and Marinescu [34, 35]. Also, we show that $\omega\left(\nabla^{F}, g^{F_{p}}\right)$ and its covariant derivatives can be expressed as Toeplitz operators.

In $\S 9.9$, we extend the results of $\S 6.1$; i.e., we compute the asymptotics of the forms $h\left(\nabla^{F_{p}}, g^{F_{p}}\right)$.

In $\S 9.10$, we show that, under our nondegeneracy assumption, we still have a spectral gap as in $\S 4.3$.

In $\S 9.11$, we give a formula for the operator $L_{t}^{F}$.

In $\S 9.12$, we extend the results of $\S 6.5$; i.e., we obtain the asymptotics of the forms $h\left(A^{\prime}, g_{t}^{\Omega^{\prime}\left(X, F_{p} \mid X\right)}\right)$.

In $\S 9.13$, under a nondegeneracy assumption, we obtain the asymptotics of the analytic torsion forms; i.e., we extend the results of $\S 7$.

Finally, in $\S 9.14$, we verify explicitly that the forms obtained in $\S 2$ are special cases of the forms that are considered here. 


\subsection{The case of a general Lie group $G$}

Let $G$ be a Lie group, which is no longer assumed to be reductive or connected. Let $N$ be a compact complex manifold as in $\S 1.4$. We assume that $G$ acts holomorphically on $N$, and that this action lifts to a holomorphic action on the line bundle $L$.

We take the manifold $M$ as in $\S 2.1$. Let $p: P_{G} \rightarrow M$ be a principal $G$-bundle, which is equipped with a flat connection. We define the manifold $\mathcal{N}$ by the obvious analogue of (1.82); i.e.,

$$
\mathcal{N}=P_{G} \times_{G} N
$$

The manifold $\mathcal{N}$ is equipped with a line bundle that is still denoted $L$. We still denote by $q$ the projection $\mathcal{N} \rightarrow M$ with fibre $N$. To this projection $q$, we can apply the formalism of $\S 2.1$. In particular, the horizontal bundle $T_{0}^{H} \mathcal{N} \subset T \mathcal{N}$ is determined by the flat connection of $P_{G}$. Let $T N$ be the holomorphic relative tangent bundle, and let $T_{\mathbf{R}} N=T \mathcal{N} / M$ denote the corresponding real bundle. Let $J^{N}$ denote the complex structure of $T_{\mathbf{R}} N$.

Using the horizontal vector bundle $T_{0}^{H} \mathcal{N}=q^{*} T M$, we get the isomorphism of vector bundles on $\mathcal{N}$,

$$
T \mathcal{N}=q^{*} T M \oplus T_{\mathbf{R}} N
$$

If $U \in T M$, let $U_{0}^{H} \in T_{0}^{H} \mathcal{N}$ denote the horizontal lift of $U$.

By (9.2), we get the isomorphism

$$
\Lambda^{*}\left(T^{*} \mathcal{N}\right)=q^{*} \Lambda^{*}\left(T^{*} M\right) \widehat{\otimes} \Lambda\left(T_{\mathbf{R}}^{*} N\right) .
$$

Forms in the first factor in the right-hand side of (9.3) will be called horizontal, and those in the second factor vertical. Forms in $q^{*} \Lambda^{i}\left(T^{*} M\right) \widehat{\otimes} \Lambda^{j}\left(T_{\mathbf{R}}^{*} N\right)$ will be said to be of type $(i, j)$.

Let $g^{T N}$ be a smooth Hermitian metric on $T N$, and let $d v_{N}$ be the corresponding

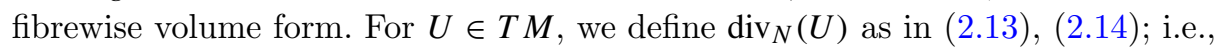

$$
L_{U_{0}^{H}} d v_{N}=\operatorname{div}_{N}(U) d v_{N} .
$$

Let $g^{L}$ be a smooth Hermitian metric on the line bundle $L$ over $\mathcal{N}$. Let $\nabla^{L}$ be the fibrewise holomorphic Hermitian connection on $L$. We can extend $\nabla^{L}$ to a connection $\nabla^{L}$ on $L$ by using the flat connection on $P_{G}$. This connection is in general nonunitary. For $U \in T M$, set

For $U \in T M$, put

$$
\omega\left(L, g^{L}\right)(U)=\left(g^{L}\right)^{-1} \nabla_{U_{0}^{H}}^{L} g^{L}
$$

$$
\nabla_{U_{0}^{H}}^{L, u}=\nabla_{U_{0}^{H}}^{L}+\frac{1}{2} \omega\left(L, g^{L}\right)(U)
$$

By combining $\nabla^{L}$ along the fibres $N$ with $\nabla^{L, u}$ in (9.6), we obtain a unitary connection on $L$, which is still denoted $\nabla^{L, u}$. Let $r^{L}, r^{L, u}$ be the curvatures of $\nabla^{L}, \nabla^{L, u}$.

Let $\bar{\partial}^{N}, \partial^{N}$ be the Dolbeault operators along the fibres $N$, let $d^{N}$ denote the corresponding fibrewise de Rham operator, and let $d^{M}$ denote the de Rham operator on $M$. Then $d^{M}$ lifts to a horizontal operator on $\mathcal{N}$. Moreover, $r^{L}$ does not have components of type $(2,0)$, and

$$
r^{L,(1,1)}=-\partial^{N} \omega\left(L, g^{L}\right) .
$$


Moreover,

$$
r^{L, u}=r^{L}+\frac{1}{2} d^{N} \omega\left(L, g^{L}\right)
$$

By the above, we get

$$
r^{L, u,(2,0)}=0, \quad r^{L, u,(1,1)}=\frac{1}{2}\left(\bar{\partial}^{N}-\partial^{N}\right) \omega\left(L, g^{L}\right) .
$$

Let $c_{1}\left(L, g^{L}\right)$ be the first Chern form associated with the connection $\nabla^{L, u}$, i.e.,

$$
c_{1}\left(L, g^{L}\right)=-\frac{r^{L, u}}{2 i \pi} .
$$

Put

$$
\xi=-2 \pi c_{1}\left(L, g^{L}\right) .
$$

By (9.9), if $U \in T M, V \in T_{\mathbf{R}} N$, then

$$
\xi\left(U_{0}^{H}, V\right)=-\frac{1}{2} \nabla_{J^{N} V} \omega\left(L, g^{L}\right)(U) .
$$

\subsection{Metric properties of the projection $q$}

Let $c_{1}^{V}\left(L, g^{L}\right)$ be the restriction of $c_{1}\left(L, g^{L}\right)$ to the fibre $N$. In what follows, we assume that $c_{1}^{V}\left(L, g^{L}\right)$ is a fibrewise positive form.

Now, we proceed as in $[14, \S 1(\mathrm{c})]$. Let $T^{H} \mathcal{N} \subset T \mathcal{N}$ denote the orthogonal bundle to $T_{\mathbf{R}} N$ with respect to the form $\xi$. Then

$$
T \mathcal{N}=T^{H} \mathcal{N} \oplus T_{\mathbf{R}} N
$$

Also,

$$
T^{H} \mathcal{N}=q^{*} T M
$$

By (9.14), we deduce that

$$
\Lambda^{*}\left(T^{*} \mathcal{N}\right) \simeq q^{*} \Lambda^{*}\left(T^{*} M\right) \widehat{\otimes} \Lambda^{*}\left(T_{\mathbf{R}}^{*} N\right) .
$$

The isomorphism in (9.15) is in general distinct from the one in (9.3).

Let $\xi^{H}, \xi^{V}$ denote the restriction of $\xi$ to $T^{H} \mathcal{N}, T_{\mathbf{R}} N$. By $(9.15), \xi^{H}, \xi^{V}$ can be considered as sections of $\Lambda^{*}\left(T^{*} \mathcal{N}\right)$, and, moreover,

$$
\xi=\xi^{H}+\xi^{V} \text {. }
$$

Until further notice, we equip $T N$ with the metric $g^{T N}$ associated with the Kähler form $\xi^{V}$. Namely, if $A, A^{\prime} \in T_{\mathbf{R}} N$, then

$$
\left\langle A, A^{\prime}\right\rangle=\xi\left(J^{N} A, A^{\prime}\right) .
$$

If $\mathcal{H} \in C^{\infty}(N, \mathbf{R})$, let $\nabla \mathcal{H}$ denote the fibrewise gradient vector field of $\mathcal{H}$ with respect to the metric $g^{T N}$.

We apply the formalism of $\S \S 2.1$ and 2.2 to the triple $\left(\mathcal{N}, T^{H} \mathcal{N}, g^{T_{\mathbf{R}} N}\right)$. Let $\nabla^{T_{\mathbf{R}} N}$ be the connection on $T_{\mathbf{R}} N$ constructed as in $\S 2.2$.

If $U \in T M$, we denote by $U^{H}$ the lift of $U$ in $T^{H} \mathcal{N}$ with respect to the splitting (9.13). There is a map $s: T M \rightarrow T_{\mathbf{R}} N$ such that, if $U \in T M$,

$$
U^{H}=U_{0}^{H}+s(U) \text {. }
$$


Then $s(U)$ is determined by the fact that, if $V \in T_{\mathbf{R}} N$,

$$
\xi(s(U), V)+\xi\left(U_{0}^{H}, V\right)=0 .
$$

By (9.12), (9.17), and (9.19), we get

$$
s(U)=\frac{1}{2} \nabla \omega\left(L, g^{L}\right)(U) .
$$

By the first identity in (9.9), and by (9.19), we deduce that

$$
\xi\left(U^{H}, V^{H}\right)=-\xi(s(U), s(V))=\xi\left(U_{0}^{H}, s(V)\right)=-\xi\left(V_{0}^{H}, s(U)\right) .
$$

Equation (9.21) determines $\xi^{H}$.

We denote by $\mathcal{S}, \mathcal{T}$ the tensors considered in the above subsections that are associated with the present situation.

Let $g^{T M}$ be a metric on $T M$, and let $\nabla^{T M}$ be the Levi-Civita connection on $\left(T M, g^{T M}\right)$. Let $\nabla^{T \mathcal{N}}=q^{*} \nabla^{T M} \oplus \nabla^{T_{\mathbf{R}} N}$ be the analogue of the connection $\nabla^{T M}$ in (2.6). Then $\mathcal{T}$ is the torsion of the connection $\nabla^{T \mathcal{N}}$.

Let $\nabla^{\Lambda^{*}\left(T^{*} \mathcal{N}\right)}$ be the connection induced by $\nabla^{T \mathcal{N}}$ on $\Lambda^{*}\left(T^{*} \mathcal{N}\right)$. Classically,

$$
d^{\mathcal{N}}=\nabla^{\Lambda \cdot\left(T^{*} \mathcal{N}\right)}+i_{\mathcal{T}}
$$

Since $\xi$ is closed, we get

$$
\left(\nabla^{\Lambda^{*}\left(T^{*} \mathcal{N}\right)}+i_{\mathcal{T}}\right) \xi=0
$$

By splitting (9.23) according to their type with respect to the factorization in (9.15), as in [14, Theorem 1.7], we get

$$
\begin{aligned}
& \left(\nabla^{\Lambda^{\cdot}\left(T_{\mathbf{R}}^{*} N\right)}+i_{\mathcal{T}}\right) \xi^{V}=0 \quad \text { on } T^{H} \mathcal{N} \times T_{\mathbf{R}} N \times T_{\mathbf{R}} N, \\
& \nabla^{\Lambda^{\cdot}\left(T^{*} M\right)} \xi^{H}=0 \quad \text { on } T^{H} \mathcal{N} \times T^{H} \mathcal{N} \times T^{H} \mathcal{N}, \\
& \nabla^{\Lambda^{\cdot}\left(T^{*} M\right)} \xi^{H}+i_{\mathcal{T}} \xi^{V}=0 \text { on } T^{H} \mathcal{N} \times T^{H} \mathcal{N} \times T_{\mathbf{R}} N
\end{aligned}
$$

The main difference with [14] lies in the first identity in (9.24). In this reference, the connection $\nabla^{T_{\mathbf{R}} N}$ preserves the complex structure of $T_{\mathbf{R}} N$, so that $\xi^{V}$ is parallel with respect to $\nabla^{\Lambda^{\cdot}\left(T_{\mathbf{R}}^{*}{ }^{N)}\right.}$, and the first identity in (9.24) splits.

Let $d^{N}$ denote the fibrewise de Rham operator along the fibres $N$.

Definition 9.1. If $\mathcal{H} \in C^{\infty}(N, \mathbf{R})$, let $X_{\mathcal{H}}$ be the fibrewise Hamiltonian vector field, so that

$$
d^{N} \mathcal{H}+i_{X_{\mathcal{H}}} \xi^{V}=0
$$

Equation (9.25) is equivalent to

$$
\left(d^{N}+i_{X_{\mathcal{H}}}\right)\left(\mathcal{H}+\xi^{V}\right)=0
$$

Recall that, if $U \in T M$, the Lie derivative operator $L_{U^{H}}$ acts naturally on smooth sections of $\Lambda^{\cdot}\left(T_{\mathbf{R}}^{*} N\right)$. Now, we prove a version of [14, Remark 1.8]. 
Proposition 9.2. If $U \in T M$, then

$$
L_{U^{H}} \xi^{V}=0 .
$$

If $U, V \in T M$, the fibrewise vector field $\mathcal{T}\left(U^{H}, V^{H}\right)$ is the Hamiltonian vector field associated with the Hamiltonian $\xi^{H}(U, V)$.

Proof. Since $i_{U^{H}} \xi^{V}=0$, we get

$$
L_{U^{H}} \xi^{V}=i_{U^{H}} d^{\mathcal{N}} \xi^{V} .
$$

By (9.22), and by the first identity in (9.24), we get (9.27). The second part of our proposition is just a reformulation of the third identity in (9.24).

By (9.19), (9.25), if $U \in T M$,

$$
\nabla_{s(U)} \mathcal{H}=\xi\left(X_{\mathcal{H}}, U_{0}^{H}\right) .
$$

\subsection{The bundle of Poisson algebras}

If $\mathcal{H}, \mathcal{H}^{\prime} \in C^{\infty}(N, \mathbf{R})$, we define the Poisson bracket $\left\{\mathcal{H}, \mathcal{H}^{\prime}\right\} \in C^{\infty}(N, \mathbf{R})$ by the formula

$$
\left\{\mathcal{H}, \mathcal{H}^{\prime}\right\}=\xi^{V}\left(X_{\mathcal{H}}, X_{\mathcal{H}^{\prime}}\right)=X_{\mathcal{H}} \mathcal{H}^{\prime}=-X_{\mathcal{H}^{\prime}} \mathcal{H} .
$$

Then \{\} is a Lie bracket. Let $\mathcal{A}$ be the bundle of Lie algebras $C^{\infty}(N, \mathbf{R})$ equipped with this Lie bracket. Let $\mathcal{V}$ be the Lie algebra of smooth vector fields along the fibre $N$. Then the map $\mathcal{H} \in \mathcal{A} \rightarrow X_{\mathcal{H}} \in \mathcal{V}$ is a morphism of bundles of Lie algebras. If $\mathcal{H} \in \mathcal{A}$, we denote by $\operatorname{ad}(\mathcal{H})$ the endomorphism of $\mathcal{A}$ that is given by $\mathcal{H}^{\prime} \rightarrow\left\{\mathcal{H}, \mathcal{H}^{\prime}\right\}$.

Note that $\mathcal{A}$ is a commutative algebra for the multiplication of functions. This structure of $\mathcal{A}$ as a commutative algebra will be called the standard structure.

Theorem 9.3. If $U, V \in T M$,

$$
\xi\left(U^{H}, V^{H}\right)=-\frac{1}{4}\left\{\omega\left(L, g^{L}\right)(U), \omega\left(L, g^{L}\right)(V)\right\} .
$$

Proof. By (9.21),

$$
\xi\left(U^{H}, V^{H}\right)=-\xi\left(J^{N} s(U), J^{N} s(V)\right) .
$$

By (9.20), (9.30), and (9.32), we get (9.31).

Remark 9.4. Let $U \mathcal{A}$ denote the enveloping algebra of $\mathcal{A}$. We denote by $*$ the product in $U \mathcal{A}$. In what follows, we will often write (9.31) in the form

$$
\xi^{H}=-\frac{1}{4} \omega\left(L, g^{L}\right)^{* 2} .
$$

Here, the star $*$ indicates that the underlying Lie bracket is the Poisson bracket.

Set

$$
\vartheta=-\frac{1}{2} \omega\left(L, g^{L}\right)
$$


Then $\vartheta$ is a section of $T^{*} M \otimes \mathcal{A}$. By (9.20), if $U \in T M$,

$$
s(U)=-\nabla \vartheta(U) .
$$

We can rewrite (9.33) in the form

$$
\xi^{H}=-\vartheta^{* 2} .
$$

From now on, $d v_{N}$ denotes the volume form with respect to the metric $g^{T N}$ associated with $\xi^{V}$.

Definition 9.5. For $\mathcal{H} \in \mathcal{A}$, put

$$
\operatorname{Tr}[\mathcal{H}]=(2 \pi)^{-n} \int_{N} \mathcal{H} d v_{N}=\int_{N} \mathcal{H} \exp \left(c_{1}^{V}\left(L, g^{L}\right)\right) .
$$

We have the trivial identity

$$
\int_{N}\left\{\mathcal{H}, \mathcal{H}^{\prime}\right\} d v_{N}=0
$$

Equation (9.38) says that $\operatorname{Tr}$ is indeed a trace on $\mathcal{A}$; i.e., it vanishes on the Poisson brackets.

Let $\operatorname{Vol}(N)$ be the symplectic volume of the fibres $N$ with respect to the symplectic form $c_{1}^{V}\left(L, g^{L}\right)$. Then $\operatorname{Vol}(N)$ is a constant. Moreover, we have the identity

$$
\operatorname{Tr}[1]=\operatorname{Vol}(N) \text {. }
$$

Definition 9.6. If $\mathcal{H}$ is a smooth section of $\mathcal{A}$, if $U \in T M$, set

$$
\nabla_{U}^{\mathcal{A}} \mathcal{H}=L_{U_{0}^{H}} \mathcal{H}, \quad \nabla_{U}^{\mathcal{A}, u} \mathcal{H}=L_{U^{H}} \mathcal{H}
$$

Then $\nabla^{\mathcal{A}}, \nabla^{\mathcal{A}, u}$ are connections on the vector bundle $\mathcal{A}$.

Theorem 9.7. If $U \in T M$, if $\mathcal{H}$ is a smooth section of $\mathcal{A}$, then

$$
\nabla_{U}^{\mathcal{A}, u} \mathcal{H}=\nabla_{U}^{\mathcal{A}} \mathcal{H}+\xi\left(X_{\mathcal{H}}, U_{0}^{H}\right) .
$$

The connection $\nabla^{\mathcal{A}, u}$ is a connection on the bundle of algebras $\mathcal{A}$ with respect to the standard structure and the Poisson structure. Namely if $\mathcal{H}, \mathcal{H}^{\prime}$ are smooth sections of $\mathcal{A}$, then

$$
\begin{aligned}
& \nabla_{U}^{\mathcal{A}, u}\left(\mathcal{H} \mathcal{H}^{\prime}\right)=\nabla_{U}^{\mathcal{A}, u} \mathcal{H} \mathcal{H}^{\prime}+\mathcal{H} \nabla_{U}^{\mathcal{A}, u} \mathcal{H}^{\prime}, \\
& \nabla_{U}^{\mathcal{A}, u}\left\{\mathcal{H}, \mathcal{H}^{\prime}\right\}=\left\{\nabla_{U}^{\mathcal{A}, u} \mathcal{H}, \mathcal{H}^{\prime}\right\}+\left\{\mathcal{H}, \nabla_{U}^{\mathcal{A}, u} \mathcal{H}^{\prime}\right\} .
\end{aligned}
$$

Moreover, $\operatorname{Tr}$ is parallel with respect to $\nabla^{\mathcal{A}, u}$, i.e.

$$
\nabla_{U} \operatorname{Tr}[\mathcal{H}]=\operatorname{Tr}\left[\nabla_{U}^{\mathcal{A}, u} \mathcal{H}\right]
$$

The connection $\nabla^{\mathcal{A}}$ is flat. The curvature $\nabla^{\mathcal{A}, u, 2}$ of $\nabla^{\mathcal{A}, u}$ is given by

$$
\nabla^{\mathcal{A}, u, 2}=\operatorname{ad}\left(\vartheta^{* 2}\right)
$$

Finally,

$$
\nabla^{\mathcal{A}} \vartheta=0, \quad \nabla^{\mathcal{A}, u} \vartheta=0
$$


Proof. Clearly,

$$
\nabla_{U}^{\mathcal{A}, u} \mathcal{H}=\nabla_{U}^{\mathcal{A}} \mathcal{H}+\nabla_{s(U)} \mathcal{H}
$$

By (9.29), (9.46), we get (9.41).

The first equation in (9.42) is trivial. By (9.25), (9.27), we get

$$
d^{N} L_{U^{H}} \mathcal{H}+i_{L_{U} X_{\mathcal{H}}} \xi^{V}=0 .
$$

By (9.30), (9.40), and (9.47), we get the second equation in (9.42). Equation (9.43) follows from similar arguments.

Clearly, the curvature of $\nabla^{\mathcal{A}}$ vanishes. Moreover, an easy computation shows that if $U, V \in T M$,

$$
\nabla^{\mathcal{A}, u, 2}(U, V) \mathcal{H}=-\nabla_{\mathcal{T}\left(U^{H}, V^{H}\right)} \mathcal{H}
$$

By Proposition 9.2, by (9.30), and (9.48), we get

$$
\nabla^{\mathcal{A}, u, 2}=-\operatorname{ad}\left(\xi^{H}\right) .
$$

By (9.36), (9.49), we get (9.44).

By (9.5), we get the first equation in (9.45). By (9.35), if $U, V \in T M$,

$$
\nabla_{s(U)} \vartheta(V)=-\langle s(U), s(V)\rangle .
$$

By (9.50), we get

$$
\nabla_{s(U)} \vartheta(V)-\nabla_{s(V)} \vartheta(U)=0 .
$$

The second equation in (9.45) follows from (9.51). The proof of our theorem is completed.

Remark 9.8. When replacing $\theta^{\mathfrak{p}}$ by $\vartheta$, (9.44) is an analogue of (4.5), and the second equation in (9.45) is an analogue of (1.41).

We have the obvious Bianchi identity

$$
\left\{\vartheta, \vartheta^{* 2}\right\}=0
$$

Definition 9.9. Set $\mathcal{A}_{\mathbf{C}}=\mathcal{A} \otimes_{\mathbf{R}} \mathbf{C}$. Let $\nabla^{\mathcal{A}_{\mathbf{C}}, u}$ be the connection induced by $\nabla^{\mathcal{A}, u}$ on $\mathcal{A}_{\mathbf{C}}$. Let $\nabla^{\mathcal{A}_{\mathbf{C}}{ }^{\prime}}$ be the connection on $\mathcal{A}_{\mathbf{C}}$ that is given by

$$
\nabla^{\mathcal{A}_{\mathbf{C}}{ }^{\prime}}=\nabla^{\mathcal{A}_{\mathbf{C}}, u}+i \operatorname{ad}(\vartheta) .
$$

Proposition 9.10. The connection $\nabla^{\mathcal{A}_{\mathbf{C}}{ }^{\prime}}$ is flat.

Proof. This is a trivial consequence of equations (9.44) and (9.45) in Theorem 9.7.

Now, we prove a counterpart to Proposition 1.12 .

Proposition 9.11. The integral along the fibre $q_{*}\left[\exp \left(c_{1}\left(L, g^{L}\right)\right)\right]$ is a constant. More precisely,

$$
q_{*}\left[\exp \left(c_{1}\left(L, g^{L}\right)\right)\right]=\operatorname{Vol}(N) .
$$


Proof. To prove our proposition, we may as well replace $c_{1}\left(L, g^{L}\right)$ by $-\xi$. Clearly,

$$
q_{*}\left[\exp \left(c_{1}\left(L, g^{L}\right)\right)\right]=\operatorname{Tr}\left[\exp \left(-\xi^{H} / 2 \pi\right)\right] .
$$

By (9.36), we can rewrite (9.55) in the form

$$
q_{*}\left[\exp \left(c_{1}\left(L, g^{L}\right)\right)\right]=\operatorname{Tr}\left[\exp \left(\vartheta^{* 2} / 2 \pi\right)\right]
$$

Using the Bianchi identity in (9.52) and the fact that $\operatorname{Tr}$ is a trace, we get

$$
\frac{\partial}{\partial s} \operatorname{Tr}\left[\exp \left(s \vartheta^{* 2}\right)\right]=\operatorname{Tr}\left[\vartheta^{* 2} \exp \left(s \vartheta^{* 2}\right)\right]=\frac{1}{2} \operatorname{Tr}\left[\left\{\vartheta, \vartheta \exp \left(s \vartheta^{* 2}\right)\right\}\right]=0 .
$$

By (9.57), we find that

$$
\operatorname{Tr}\left[\exp \left(\vartheta^{* 2} / 2 \pi\right)\right]=\operatorname{Tr}[1] .
$$

By (9.39), (9.56), and (9.58), we get (9.54).

Remark 9.12. The proof of Proposition 9.11 closely resembles part of the standard proof of the index theorem [10, proof of Theorem 3.1]. Using (9.9) and the fact that $\xi^{V}$ is $\bar{\partial}^{N}$ and $\partial^{N}$ closed, one can also give another proof of Proposition 9.11. The fundamental fact about the proof of Proposition 9.11 is that it involves a subtle extra structure on the fibre $N$.

\subsection{The forms $a_{t}, b_{t}$}

We introduce a Euclidean vector bundle with connection $\widehat{E}$ on $M$ as in $\S 1.7$. Let $\beta$ be a smooth section of $\widehat{E}^{*} \otimes \mathcal{A}$. We use otherwise exactly the same notation as in $\S 1.7$, simply replacing $\mathfrak{g}_{r}$ by $\mathcal{A}$.

In what follows, the product in $\mathcal{A}$ will be the standard product of smooth functions in $C^{\infty}(N, \mathbf{R})$. Here, we define $\sigma\left(\mathcal{A}_{t}^{2}\right)$ by the formula

$$
\sigma\left(\mathcal{A}_{t}^{2}\right)=\frac{1}{4}\left|\widehat{e}_{i}, R^{\widehat{E}} \widehat{e}_{j}\right| \widehat{e}^{i} \widehat{e}^{j}-\vartheta^{* 2}+\sqrt{t} \nabla_{.}^{\widehat{E} \otimes \mathcal{A}, u} \beta+t|\beta|^{2}-t \beta^{* 2} .
$$

Then $\sigma\left(\mathcal{A}_{t}^{2}\right)$ is a smooth section of $\Lambda^{*}\left(T^{*} M\right) \widehat{\otimes} \Lambda^{*}\left(\widehat{E}^{*}\right) \otimes \mathcal{A}$.

Set

$$
\mathcal{L}_{t}=\nabla^{\widehat{E} \otimes \mathcal{A}, u}+2 i_{\sqrt{t} \beta}-\operatorname{ad}(\sqrt{t} \beta) .
$$

We claim that the obvious analogue of Theorem 1.6 holds, so that, in particular,

$$
\mathcal{L}_{t} \sigma\left(\mathcal{A}_{t}^{2}\right)=0, \quad \frac{\partial}{\partial t} \sigma\left(\mathcal{A}_{t}^{2}\right)=\mathcal{L}_{t} \frac{\beta}{2 \sqrt{t}} .
$$

Indeed, equation (9.44) simply replaces (1.39) in the proof of Theorem 1.6.

Note that $\exp \left(-\sigma\left(\mathcal{A}_{t}^{2}\right)\right)$ is a section of the algebra $\Lambda^{\cdot}\left(T^{*} M\right) \widehat{\otimes} \Lambda^{\cdot}\left(\widehat{E}^{*}\right) \otimes \mathcal{A}$. Then $\operatorname{Tr} \int^{\widehat{B}}$ maps this algebra into $\Lambda^{*}\left(T^{*} M\right)$.

When $\beta$ is viewed as a smooth section on $\mathcal{N}$ of $q^{*} \Lambda^{\cdot}\left(\widehat{E}^{*}\right)$, we will use the notation $\sigma_{\beta}$ instead of $\beta$. In particular, while $\nabla^{\widehat{E} \otimes \mathcal{A}, u} \beta$ is a section on $M$ of $T^{*} M \otimes \widehat{E}^{*} \otimes \mathcal{A}, \nabla^{\widehat{E}} \sigma_{\beta}$ is a section on $\mathcal{N}$ of $\Lambda^{*}\left(T^{*} \mathcal{N}\right) \otimes q^{*} \widehat{E}^{*}$. 
Definition 9.13. We will say that $\beta$ is nondegenerate if $\sigma_{\beta}$ does not vanish on $\mathcal{N}$.

Let $\varphi$ be the endomorphism of $\Lambda^{*}\left(T^{*} M\right)$ mapping $\alpha \in \Lambda^{k}\left(T^{*} M\right)$ into $(2 \pi)^{-k / 2} \alpha$. Now, we imitate Definition 1.14.

Definition 9.14. For $t \geqslant 0$, set

$$
\begin{aligned}
& a_{t}=(2 \pi)^{m / 2} \underline{\varphi} \operatorname{Tr}\left[\int^{\widehat{B}} \exp \left(-\sigma\left(\mathcal{A}_{t}^{2}\right)\right)\right], \\
& b_{t}=(2 \pi)^{(m-1) / 2} \underline{\varphi} \operatorname{Tr}\left[\int^{\widehat{B}} \frac{\beta}{2 \sqrt{t}} \exp \left(-\sigma\left(\mathcal{A}_{t}^{2}\right)\right)\right] .
\end{aligned}
$$

Then $a_{t}, b_{t}$ are smooth real forms on $M$.

Recall that the forms $\underline{a}_{t}, \underline{b}_{t}$ on $\widehat{\mathcal{E}}$ were defined in Definition 1.8. Also, we identify $\widehat{E}$ and $\widehat{E}^{*}$ by the metric $g \widehat{E}$. By (1.74), as in (1.86), we get

$$
\begin{aligned}
\sigma_{\beta}^{*} \underline{a}_{t} & =\int^{\widehat{B}} \exp \left(-\frac{1}{4}\left\langle\widehat{e}_{i}, R^{\widehat{E}} \widehat{e}_{j}\right) \widehat{e}^{i} \widehat{e}^{j}-\nabla^{\widehat{E}} \sqrt{t} \sigma_{\beta}-t\left|\sigma_{\beta}\right|^{2}\right), \\
\sigma_{\beta}^{*} \underline{b}_{t} & =\int^{\widehat{B}} \frac{\sigma_{\beta}}{2 \sqrt{t}} \exp \left(-\frac{1}{4}\left\langle\widehat{e}_{i},\left.R^{E^{E}} \widehat{e}_{j}\left|\widehat{e}^{i} \widehat{e}^{j}-\nabla^{\widehat{E}} \sqrt{t} \sigma_{\beta}-t\right| \sigma_{\beta}\right|^{2}\right) .\right.
\end{aligned}
$$

Recall that the current $\psi$ on $\widehat{\mathcal{E}}$ was defined in Definition 1.9. We establish an extension of Theorem 1.15.

Theorem 9.15. For any $t \geqslant 0$, the form $a_{t}$ is closed, and its cohomology class does not depend on $t \geqslant 0$. We have the identity

$$
\frac{\partial}{\partial t} a_{t}=-d b_{t}
$$

Moreover,

$$
a_{t}=q_{*}\left[\sigma_{\beta}^{*} \underline{a}_{t} \exp \left(c_{1}\left(L, g^{L}\right)\right)\right], \quad b_{t}=q_{*}\left[\sigma_{\beta}^{*} \underline{b}_{t} \exp \left(c_{1}\left(L, g^{L}\right)\right)\right] .
$$

Also,

$$
a_{0}=e\left(\widehat{E}, \nabla^{\widehat{E}}\right) q_{*}\left[\exp \left(c_{1}\left(L, g^{L}\right)\right)\right]=e\left(\widehat{E}, \nabla^{\widehat{E}}\right) q_{*}\left[\exp \left(c_{1}^{V}\left(L, g^{L}\right)\right)\right] .
$$

If $\beta$ is nondegenerate, for any compact set $K \subset M$, there exists $c_{K}>0$ such that, on $K$, as $t \rightarrow+\infty$,

$$
a_{t}=\mathcal{O}\left(e^{-c_{K} t}\right), \quad b_{t}=\mathcal{O}\left(e^{-c_{K} t}\right) .
$$

If $\beta$ is nondegenerate, the Euler class $e(\widehat{E})$ vanishes. Also, $\sigma_{\beta}^{*} \psi$ is a smooth $(m-1)$-form on $\mathcal{N}$ with values in $o(\widehat{E})$ such that

$$
d \sigma_{\beta}^{*} \psi=q^{*} e\left(\widehat{E}, \nabla^{\widehat{E}}\right) .
$$


Proof. The proof of the first part of our theorem is formally the same as the proof of Theorem 1.15. Instead of using the fact that $R$ is $G$-invariant, so that its derivative vanishes when it is evaluated on commutators, we use instead the fact that $\mathrm{Tr}$ is a trace on the algebra $\mathcal{A}$, so that (9.38) holds. When combined with (9.61), we get the first part of our theorem.

Now, we establish (9.65). We denote with subscripts $H, V$ differentiation on $T^{H} \mathcal{N}, T_{\mathbf{R}} N$. Then

$$
\nabla^{\widehat{E}} \sigma_{\beta}=\nabla_{H}^{\widehat{E}} \sigma_{\beta}+\nabla_{V}^{\widehat{E}} \sigma_{\beta}
$$

The term $\nabla_{H}^{\widehat{E}} \sigma_{\beta}$ is responsible for the appearance of $\nabla^{\widehat{E} \otimes \mathcal{A}, u} \beta$ when evaluating the right-hand side of (9.65). So we concentrate on $\nabla_{V}^{\widehat{E}} \sigma_{\beta}$. Set

$$
i_{X_{\sigma_{\beta}}}=\widehat{e}^{i} i_{X_{\beta}\left(\widehat{e}_{i}\right)}
$$

By (9.25), we get

$$
\nabla_{V}^{\widehat{E}} \sigma_{\beta}=i_{X_{\sigma_{\beta}}} \xi^{V}
$$

Now, we proceed exactly as in (1.99)-(1.101), and we get (9.65).

The first identity in (9.66) is obvious, and the second identity follows from Proposition 9.11. Equation (9.67) is trivial. Equation (9.68) follows from (1.78). The proof of our theorem is completed.

\subsection{The forms $c_{t}, d_{t}$ on $M$}

We make the same assumptions as in $\S \S 2.1$ and 2.2 in the context of $\S 9.4$. Also, we take $\widehat{E}$ to be the Euclidean vector bundle $\widehat{T X}$ as in $\S 2.3$. We denote by $\widehat{\vartheta}$ the section of $\widehat{T^{*} X} \otimes \mathcal{A}$ that is obtained by restriction of $\vartheta$ to $T X \simeq \widehat{T X}$. In what follows, we take $\beta=\widehat{\vartheta}$. By (9.59), (9.60), we get

$$
\begin{aligned}
& \sigma\left(\mathcal{A}_{t}^{2}\right)=\frac{1}{4}\left\langle e_{i},\left.R^{T X} e_{j}\left|\widehat{e}^{i} \widehat{e}^{j}-\vartheta * 2+\sqrt{t} \nabla^{\widehat{T X} \otimes \mathcal{A}, u} \widehat{\vartheta}+t\right| \widehat{\vartheta}\right|^{2}-t \widehat{\vartheta}^{* 2},\right. \\
& \mathcal{L}_{t}=\nabla^{T X} \otimes \mathcal{A}, u+2 i \sqrt{t \vartheta}-\operatorname{ad}(\sqrt{t} \widehat{\vartheta}) .
\end{aligned}
$$

By (9.61), we get

$$
\mathcal{L}_{t} \sigma\left(\mathcal{A}_{t}^{2}\right)=0, \quad \frac{\partial}{\partial t} \sigma\left(\mathcal{A}_{t}^{2}\right)=\mathcal{L}_{t} \frac{\widehat{\vartheta}}{2 \sqrt{t}} .
$$

Let $\vartheta^{X}$ be the section of $T^{*} X \otimes \mathcal{A}$ obtained by restriction of $\vartheta$ to $T X$, and let $\vartheta^{H}$ be the section of $\pi^{*} T^{*} S \otimes \mathcal{A}$ obtained by restriction of $\vartheta$ to $T^{H} M$, so that

$$
\vartheta=\vartheta^{X}+\vartheta^{H}
$$

Recall that $T^{0}$ was defined in (2.22). Then $T^{0}$ is a section of $\pi^{*} \Lambda^{*}\left(T^{*} S\right) \widehat{\otimes} \Lambda^{*}\left(\widehat{T^{*} X}\right)$ with values in $T X$. The properties of $i_{T^{0}}$ were briefly described after equation (2.22). Also, the operator $\mathcal{N}^{d}$ was defined in (2.27).

Definition 9.16. Set

$$
\widehat{\mathcal{L}}_{t}=\widehat{\nabla}^{T X \otimes \mathcal{A}, u}+2 i_{-\sqrt{t} \vartheta} \vartheta^{\frac{1}{2}} T^{0}+\frac{\operatorname{ad}(\vartheta)}{\sqrt{t}} .
$$


Now, we establish an analogue of Theorem 2.4.

Theorem 9.17. The following identity holds:

$$
\begin{aligned}
\sigma\left(\mathcal{A}_{t}^{2}\right)= & \frac{1}{4}\left\langle e_{i}, \widehat{R}^{T X} e_{j}\right) e^{i} e^{j}+\widehat{\nabla}^{T X \otimes \mathcal{A}, u}\left(-\sqrt{t} \vartheta^{X}+\frac{1}{2} T^{0}\right) \\
& +\left|-\sqrt{t} \vartheta^{X}+\frac{1}{2} T^{0}\right|^{2}-t \widehat{\vartheta}^{* 2}-\vartheta^{* 2}-\widehat{\nabla}^{T X \otimes \mathcal{A}, u} \sqrt{t} \vartheta^{H}-\frac{1}{4} \widehat{d}^{X} \widehat{T}^{H} .
\end{aligned}
$$

Moreover, for $t>0$,

$$
\begin{gathered}
\widehat{\mathcal{L}}_{t} \sigma\left(\mathcal{A}_{t}^{2}\right)=0, \\
\frac{\partial}{\partial t} \sigma\left(\mathcal{A}_{t}^{2}\right)=-\widehat{\mathcal{L}}_{t} \frac{\vartheta}{2 \sqrt{t}}-\widehat{\vartheta}^{* 2}+\frac{\vartheta^{* 2}}{t}, \\
\frac{\partial}{\partial t} \sigma\left(\mathcal{A}_{t}^{2}\right)+\frac{1}{2 t}\left[\mathcal{N}^{d}, \sigma\left(\mathcal{A}_{t}^{2}\right)\right]=-\widehat{\mathcal{L}}_{t} \frac{\vartheta}{2 \sqrt{t}} .
\end{gathered}
$$

Finally,

$$
\begin{aligned}
& -\mathcal{L}_{t} \vartheta=t \widehat{\mathcal{L}_{t}} \widehat{\vartheta}=\sqrt{t}\{\widehat{\vartheta}, \vartheta\} \\
& \mathcal{L}_{t} \widehat{\vartheta}+\widehat{\mathcal{L}}_{t} \vartheta=2\left(-\sqrt{t} \widehat{\vartheta}^{* 2}+\frac{\vartheta^{* 2}}{\sqrt{t}}\right)
\end{aligned}
$$

Proof. By (9.45), instead of (2.33), we get

$$
\left(\nabla^{\Lambda^{\cdot}\left(T^{*} M\right) \otimes \mathcal{A}, u}+i_{T}\right) \vartheta=0 .
$$

By (9.74), (9.79), instead of (2.34), we get

$$
\nabla^{\widehat{T X} \otimes \mathcal{A}, u} \widehat{\vartheta}=-\left(\widehat{\nabla}^{T X \otimes \mathcal{A}, u}+i_{T^{0}}\right) \vartheta^{X}-\widehat{\nabla}^{T X \otimes \mathcal{A}, u} \vartheta^{H} .
$$

By (2.26), (9.72), and (9.80), we get (9.76).

Now, we establish (9.77). By (1.73), (9.44), instead of (2.35), we get

$$
\begin{aligned}
& \left(\widehat{\nabla}^{T X \otimes \mathcal{A}, u}+2 i_{-\sqrt{t} \vartheta^{X}+\frac{1}{2} T^{0}}\right)\left(\frac{1}{4}\left\langle e_{i}, \widehat{R}^{T X} e_{j}\right) e^{i} e^{j}\right. \\
& \left.\quad+\widehat{\nabla}^{T X \otimes \mathcal{A}, u}\left(-\sqrt{t} \vartheta^{X}+\frac{1}{2} T^{0}\right)+\left|-\sqrt{t} \vartheta^{X}+\frac{1}{2} T^{0}\right|^{2}\right)=-\left\{\widehat{\vartheta}^{* 2}, \sqrt{t} \vartheta^{X}\right\} .
\end{aligned}
$$

By (9.45),

$$
\widehat{\nabla}^{T X \otimes \mathcal{A}, u} \widehat{\vartheta}=0 .
$$

By Theorem 9.7, instead of (2.37), we obtain

$$
\widehat{\nabla}^{T X \otimes \mathcal{A}, u} \vartheta^{* 2}=\left\{\widehat{\nabla}^{T X \otimes \mathcal{A}, u} \vartheta, \vartheta\right\} .
$$

Instead of (2.38), we have the identity

$$
-2 i_{-\sqrt{t} \vartheta^{X}+\frac{1}{2} T_{0}} \vartheta^{* 2}+\left\{\frac{\vartheta}{\sqrt{t}},\left|-\sqrt{t} \vartheta^{X}+\frac{1}{2} T^{0}\right|^{2}\right\}=0 .
$$

By (9.76), and by (9.80)-(9.84), we get the first identity in (9.77). 
By (9.76), we obtain

$$
\frac{\partial}{\partial t} \sigma\left(\mathcal{A}_{t}^{2}\right)=-\frac{1}{2 \sqrt{t}} \widehat{\nabla}^{T X \otimes \mathcal{A}, u} \vartheta-\frac{1}{\sqrt{t}} i_{-\sqrt{t} \vartheta^{X}+\frac{1}{2} T^{0} \vartheta-\widehat{\vartheta}^{* 2},}
$$

which is equivalent to the second identity in (9.77). The third identity in (9.77) follows from (9.72) and from the second identity in (9.77).

By (9.45), (9.72), we get one identity in the first line of (9.78). By (9.75), (9.82), we obtain the other identity. By (9.73), (9.77), we get the last identity in (9.78).

Now, we have the analogue of Theorem 2.5.

Theorem 9.18. For any $t \geqslant 0$, the following identities hold:

$$
\pi_{*} a_{t}=\chi(X) \operatorname{Vol}(N), \quad \pi_{*} b_{t}=0 .
$$

Proof. Using Theorem 9.17, the proof is identical to the proof of Theorem 2.5.

Now, we prove a counterpart to Proposition 2.7. Let $\gamma$ be the odd form on $M$,

$$
\gamma=-\sqrt{2 \pi} \underline{\varphi} \operatorname{Tr}\left[\vartheta \exp \left(\vartheta^{* 2}\right)\right]
$$

Proposition 9.19. The form $\gamma$ is closed. Moreover,

$$
\gamma=-q_{*}\left[\vartheta \exp \left(c_{1}\left(L, g^{L}\right)\right)\right]
$$

Proof. By (9.43) and (9.45), the form $\gamma$ is closed. By (9.11), (9.36), and (9.87), we get (9.88).

Recall that $L$ was defined in (2.54). Now, we imitate Definition 2.9.

Definition 9.20. Let $c_{t}, d_{t}, e_{t}$ be the forms on $M$,

$$
\begin{gathered}
c_{t}=-(2 \pi)^{(m+1) / 2} \underline{\varphi} \operatorname{Tr}\left[\int^{\widehat{B}} \vartheta \exp \left(-\sigma\left(\mathcal{A}_{t}^{2}\right)\right)\right], \\
d_{t}=-(2 \pi)^{m / 2} \underline{\varphi} \operatorname{Tr}\left[\int^{\widehat{B}} \sqrt{t} \frac{\vartheta \wedge \widehat{\vartheta}}{2} \exp \left(-\sigma\left(\mathcal{A}_{t}^{2}\right)\right)\right], \\
e_{t}=(2 \pi)^{m / 2} \underline{\varphi} \operatorname{Tr}\left[\int^{\widehat{B}} \frac{L}{4 \sqrt{t}} \exp \left(-\sigma\left(\mathcal{A}_{t}^{2}\right)\right)\right] .
\end{gathered}
$$

Recall that $\widehat{\mathcal{X}}$ is the total space of $\widehat{T X}$. Then $\underline{a}_{t}, \underline{b}_{t}$ are forms on $\widehat{\mathcal{X}}$.

We have the following analogue of Theorem 2.10 .

Theorem 9.21. The forms $\pi_{*} c_{t}$ are odd, they are closed, and their cohomology class does not depend on $t \geqslant 0$. The forms $\pi_{*} d_{t}, \pi_{*} e_{t}$ are even. Moreover,

$$
\pi_{*} c_{0}=\pi_{*}\left[e\left(T X, \nabla^{T X}\right) \gamma\right] .
$$


Also,

$$
\frac{\partial}{\partial t} \pi_{*} c_{t}=\frac{1}{t} d \pi_{*} d_{t}, \quad\left(1+2 t \frac{\partial}{\partial t}\right) \psi_{\sqrt{t}} \pi_{*} e_{t}=\psi_{\sqrt{t}} \pi_{*} d_{t}
$$

Moreover,

$$
c_{t}=-q_{*}\left[\vartheta \sigma_{\widehat{\vartheta}}^{*} \underline{a}_{t} \exp \left(c_{1}\left(L, g^{L}\right)\right)\right], \quad d_{t}=-t q_{*}\left[\vartheta \sigma_{\widehat{\vartheta}}^{*} \underline{b}_{t} \exp \left(c_{1}\left(L, g^{L}\right)\right)\right] .
$$

If $\widehat{\vartheta}$ is nondegenerate, if $K$ is a compact subset of $M$, there exists $c_{K}>0$, such that, on $K$, as $t \rightarrow+\infty$,

$$
c_{t}=\mathcal{O}\left(e^{-c_{K} t}\right), \quad d_{t}=\mathcal{O}\left(e^{-c_{K} t}\right), \quad e_{t}=\mathcal{O}\left(e^{-c_{K} t}\right) .
$$

Proof. Using Theorem 9.17, and in particular (9.78), the proof of the first part of our theorem proceeds exactly as the proof of Theorem 2.10. The proof of (9.92) is the same as the proof of (9.65). Equation (9.93) is trivial. The proof of our theorem is completed.

\subsection{The $W$-invariant}

We assume $\widehat{\vartheta}$ to be nondegenerate. We identify $\widehat{T X}$ and $\widehat{T^{*} X}$ by the metric $g^{\widehat{T X}}$. By equation (9.68) in Theorem 9.15,

$$
d \sigma_{\widehat{\vartheta}}^{*} \psi=q^{*} e\left(T X, \nabla^{T X}\right) .
$$

By (1.77), by equation (9.65) in Theorem 9.15, and by Theorem 9.18, we get

$$
(\pi q)_{*}\left[\sigma_{\widehat{\vartheta}}^{*} \psi \exp \left(c_{1}\left(L, g^{L}\right)\right)\right]=0 .
$$

Definition 9.22. Set

$$
W=-\int_{0}^{+\infty} d_{t} \frac{d t}{t} .
$$

Theorem 9.23. The following identity holds:

$$
W=q_{*}\left[\vartheta \sigma_{\widehat{\vartheta}}^{*} \psi \exp \left(c_{1}\left(L, g^{L}\right)\right)\right] .
$$

The even form $\pi_{*} W$ on $S$ is such that

$$
d \pi_{*} W=\pi_{*}\left[e\left(T X, \nabla^{T X}\right) \gamma\right] .
$$

If $\operatorname{dim} X$ is odd, the form $\pi_{*} W$ is closed, and its cohomology class $\left[\pi_{*} W\right] \in H^{\cdot}(S, \mathbf{R})$ does not depend on $\left(T^{H} M, g^{T X}\right)$ or on infinitesimal variations of the metric $g^{L}$.

Proof. The first part of our theorem follows from (1.77), from Theorem 9.21, and from (9.96). When $\operatorname{dim} X$ is odd, the right-hand side of (9.98) vanishes. The second part of the theorem is now obvious.

Remark 9.24. Equation (9.98) for $d \pi_{*} W$ cannot be easily derived from equation (9.68) for $d \sigma_{\widehat{\theta}}^{*} \psi$ and from the explicit formula (9.97) for $W$. The results of $\S \S 2.7$ and 2.8 extend to this more general situation, with similar proofs. 


\subsection{The curvature of the unitary connection on $F$}

Recall that $G$ acts on $C^{\infty}(N, L)$. Set

$$
\mathcal{F}=P_{G} \times_{G} C^{\infty}(N, L), \quad F=P_{G} \times_{G} H^{(0,0)}(N, L) .
$$

Then $\mathcal{F}, F$ are complex vector bundles on $M$, which are equipped with flat connections $\nabla^{\mathcal{F}}, \nabla^{F}$. Moreover, $F$ is a flat subbundle of $\mathcal{F}$. If $s$ is a smooth section of $\mathcal{F}$, if $U \in T M$, then

$$
\nabla_{U}^{\mathcal{F}} s=\nabla_{U_{0}^{H}}^{L} s
$$

Moreover, the flat connection $\nabla^{\mathcal{F}}$ induces a corresponding flat connection $\nabla^{\mathcal{F} / F}$ on $\mathcal{F} / F$.

We assume here that $g^{T N}$ is an arbitrary Hermitian metric on $T N$. We equip the fibres $\mathcal{F}$ with the Hermitian metric $g^{\mathcal{F}}$ associated with $g^{T N}, g^{L}$ as in (3.3). Let $g^{F}$ be the corresponding Hermitian metric on $F$. Let $P$ denote the fibrewise orthogonal projection $\mathcal{F} \rightarrow F$.

If $U \in T M$, we define $\operatorname{div}_{N}(U)$ as in (9.4), so that $\operatorname{div}_{N}(U)$ depends on the choice of $g^{T N}$. In what follows, we will use the notation $d v_{N}$ for the volume form along the fibres $N$ that is associated with the symplectic form $\xi^{V}$. Ultimately, our results do not depend on the choice of $g^{T N}$, so that the reader may assume, if he/she wishes, that $g^{T N}$ is the Hermitian metric associated with $\xi^{V}$.

Let $F^{\perp}$ be the fibrewise orthogonal bundle to $F$ in $\mathcal{F}$. By identifying $F^{\perp}$ to $\mathcal{F} / F, F^{\perp}$ inherits a flat connection $\nabla^{F^{\perp}}$. One verifies easily that $\nabla^{F^{\perp}}$ is the orthogonal projection of $\nabla^{\mathcal{F}}$ on $F^{\perp}$. With respect to the splitting $\mathcal{F}=F \oplus F^{\perp}$, we can write $\nabla^{\mathcal{F}}$ in the form

$$
\nabla^{\mathcal{F}}=\left[\begin{array}{cc}
\nabla^{F} & \alpha \\
0 & \nabla^{F^{\perp}}
\end{array}\right] .
$$

We define $\omega\left(\nabla^{\mathcal{F}}, g^{\mathcal{F}}\right)$ as in (4.4); i.e.,

$$
\omega\left(\nabla^{\mathcal{F}}, g^{\mathcal{F}}\right)=\left(g^{\mathcal{F}}\right)^{-1} \nabla^{\mathcal{F}} g^{\mathcal{F}} .
$$

Let $\nabla^{\mathcal{F}, u}$ denote the unitary connection on $\mathcal{F}$ that is defined as in (4.2); i.e.,

$$
\nabla^{\mathcal{F}, u}=\nabla^{\mathcal{F}}+\frac{1}{2} \omega\left(\nabla^{\mathcal{F}}, g^{\mathcal{F}}\right)
$$

We use a similar notation for the vector bundle $F$. One finds easily that

$$
\omega\left(\nabla^{F}, g^{F}\right)=P \omega\left(\nabla^{\mathcal{F}}, g^{\mathcal{F}}\right) P, \quad \nabla^{F, u}=P \nabla^{\mathcal{F}, u} P .
$$

In what follows, []$_{+}$denotes an anticommutator.

Theorem 9.25. If $U \in T M$, then

$$
\omega\left(\nabla^{\mathcal{F}}, g^{\mathcal{F}}\right)(U)=\omega\left(L, g^{L}\right)(U)+\operatorname{div}_{N}(U) .
$$

The curvature $\nabla^{\mathcal{F}, u, 2}$ of $\nabla^{\mathcal{F}, u}$ vanishes.

Moreover,

$$
\nabla^{F, u, 2}=-\frac{1}{4}\left[P\left(\omega\left(\nabla^{\mathcal{F}}, g^{\mathcal{F}}\right)\right) P\right]^{2} .
$$

If $\mathcal{H}$ is a smooth section of $\mathcal{A}$, then

$$
\nabla^{F, u} P \mathcal{H} P=P\left(\nabla^{\mathcal{A}} \mathcal{H}\right) P+P \omega\left(\nabla^{\mathcal{F}}, g^{\mathcal{F}}\right) \mathcal{H} P-\left[P \frac{1}{2} \omega\left(\nabla^{\mathcal{F}}, g^{\mathcal{F}}\right) P, P \mathcal{H} P\right]_{+} .
$$


Proof. By (3.3), (9.4), (9.5), and (9.100), we get (9.105). By (4.5), (9.4), (9.5), and (9.105), we deduce the vanishing of $\nabla^{\mathcal{F}, u, 2}$. Equation (9.106) follows from (4.5), (9.104).

The unitary connection $\nabla^{\mathcal{F}, u}$ on $\mathcal{F}$ is characterized by the fact that $\nabla^{\mathcal{F}, u}-\nabla^{\mathcal{F}}$ is a 1-form on $M$ with values in self-adjoint endomorphisms of $\mathcal{F}$. By (9.101), we deduce that

$$
\nabla^{\mathcal{F}, u}=\left[\begin{array}{cc}
\nabla^{F, u} & \alpha / 2 \\
-\alpha^{*} / 2 & \nabla^{F^{\perp}, u}
\end{array}\right] .
$$

By (9.103), (9.108), we conclude that

$$
\alpha=-P \omega\left(\nabla^{\mathcal{F}}, g^{\mathcal{F}}\right) P^{\perp} .
$$

Now, we establish (9.107). By (9.40), (9.105), we get

$$
\nabla^{\mathcal{F}, u} \mathcal{H}=\nabla^{\mathcal{A}} \mathcal{H}
$$

By (9.108), (9.110), we deduce that

$$
P\left(\nabla^{\mathcal{A}} \mathcal{H}\right) P=\nabla^{F, u} P \mathcal{H} P+\frac{1}{2} P \alpha P^{\perp} \mathcal{H} P+\frac{1}{2} P \mathcal{H} P^{\perp} \alpha^{*} P .
$$

By (9.109), (9.111), we get (9.107). The proof of our theorem is completed.

\subsection{The algebra of Toeplitz operators}

Put $\mathcal{V}_{\mathbf{C}}=\mathcal{V} \otimes_{\mathbf{R}} \mathbf{C}$. The morphism of Lie algebras $\mathcal{H} \in \mathcal{A} \rightarrow X_{\mathcal{H}} \in \mathcal{V}$ extends to a morphism of Lie algebras from $\mathcal{A}_{\mathbf{C}}$ into $\mathcal{V}_{\mathbf{C}}$. If $Y \in \mathcal{V}_{\mathbf{C}}$, we denote by $Y^{(1,0)}, Y^{(0,1)}$ the components of $Y$ in $T N, \overline{T N}$.

For $p \in \mathbf{N}$, when replacing $L$ by $L^{p}$, we denote with a subscript $p$ the objects that were considered before.

Given $p \in \mathbf{N}$, if $A \in \operatorname{End}\left(L_{2}\left(N, L^{p}\right)\right)$, let $\|A\|$ be the norm of $A$ with respect to the Hilbert norm on $L_{2}\left(N, L^{p}\right)$. If $A$ is trace class, we denote by $\|A\|_{1}$ the norm of $A$ in the vector space of trace class operators.

Assume that $A=P_{p} A P_{p}$. Then $A$ is trace class. Moreover,

$$
\|A\|_{1} \leqslant\|A\| \operatorname{dim} F_{p} .
$$

By the formula of Riemann-Roch-Hirzebruch, there is $C>0$ such that, for $p \in \mathbf{N}$,

$$
\operatorname{dim} F_{p} \leqslant C p^{n}
$$

By (9.112), (9.113), we get

$$
\|A\|_{1} \leqslant C\|A\| p^{n}
$$

If $\mathcal{H} \in \mathcal{A}$, set

$$
\|\mathcal{H}\|_{\infty}=\sup _{z \in N}|\mathcal{H}(z)| .
$$

Then \|\|$_{\infty}$ is a norm on the bundle $\mathcal{A}$.

First, we describe the formalism discovered by Berezin [2] and Boutet de Monvel and Guillemin [25] on the definition of Toeplitz operators, and further pursued by Bordemann, 
Meinrenken, and Schlichenmaier [23], Schlichenmaier [45], and Ma and Marinescu [34, 35]. In particular, if $\mathcal{H} \in \mathcal{A}_{\mathbf{C}}, T_{\mathcal{H}, p}$ denotes the Berezin-Toeplitz quantization of $\mathcal{H}$, which is given by

$$
T_{\mathcal{H}, p}=P_{p} \mathcal{H} P_{p}
$$

Note that

$$
\left\|T_{\mathcal{H}, p}\right\| \leqslant\|\mathcal{H}\|_{\infty} .
$$

In [34, Definition 7.2.1], Ma and Marinescu defined a vector space $\mathcal{T}$ of Toeplitz operators. A Toeplitz operator is a family of bounded operators $T_{p} \in \operatorname{End}\left(L_{2}\left(N, L^{p}\right)\right), p \in$ $\mathbf{N}$ such that $T_{p}=P_{p} T_{p} P_{p}$, and that there exists a family $\mathcal{H}_{\ell} \in \mathcal{A}, \ell \in \mathbf{N}$ for which, for any $k \in \mathbf{N}$, there exists $C_{k} \in \mathbf{N}$ such that, for any $p \in \mathbf{N}^{*}$,

$$
\left\|T_{p}-\sum_{\ell=0}^{k} T_{\mathcal{H}_{\ell}, p} p^{-\ell}\right\| \leqslant C_{k} p^{-k-1} .
$$

As in [34], we use the notation

$$
T_{p}=\sum_{\ell=0}^{+\infty} T_{\mathcal{H}_{\ell}, p} p^{-\ell}+\mathcal{O}\left(p^{-\infty}\right)
$$

If we only specify the first $k$ coefficients, the sum $\sum_{\ell=0}^{+\infty}$ is replaced by $\sum_{\ell=0}^{k}$.

One result that follows from the above references is that $\mathcal{T}$ is an algebra. More precisely, Ma and Marinescu [34, Theorem 7.4.1] showed that the coefficients of the product of Toeplitz operators can be computed locally in terms of differential operators acting on the coefficients $\mathcal{H}_{\ell}$ defining the Toeplitz operators.

In [36, Theorem 0.3 and Remark 0.5], Ma and Marinescu showed that, if $\mathcal{H}, \mathcal{H}^{\prime} \in \mathcal{A}_{\mathbf{C}}$, then

$$
T_{\mathcal{H}, p} T_{\mathcal{H}^{\prime}, p}=T_{\mathcal{H} \mathcal{H}^{\prime}, p}+T_{i \xi^{V}\left(X_{\mathcal{H}}^{(0,1)}, X_{\mathcal{H}^{\prime}}^{(1,0)}\right)} p^{-1}+\mathcal{O}\left(p^{-2}\right)
$$

Let \langle\rangle denote the scalar product on $T_{\mathbf{R}} N$ that is associated with the Kähler form $\xi^{V}$. By (9.120), we deduce that

$$
\begin{gathered}
T_{\mathcal{H}, p} T_{\mathcal{H}^{\prime}, p}=T_{\mathcal{H} \mathcal{H}^{\prime}, p}+\mathcal{O}\left(p^{-1}\right), \\
{\left[T_{\mathcal{H}, p}, T_{\mathcal{H}^{\prime}, p}\right]=i T_{\left\{\mathcal{H}, \mathcal{H}^{\prime}\right\}, p} p^{-1}+\mathcal{O}\left(p^{-2}\right),} \\
{\left[T_{\mathcal{H}, p}, T_{\mathcal{H}^{\prime}, p}\right]_{+}=2 T_{\mathcal{H} \mathcal{H}^{\prime}, p}-T_{\left\langle\nabla \mathcal{H}, \nabla \mathcal{H}^{\prime}\right\rangle, p} p^{-1}+\mathcal{O}\left(p^{-2}\right) .}
\end{gathered}
$$

When the fibrewise metric $g^{T N}$ is associated with the Kähler form $\xi^{V}$, the first two equations in (9.121) were first proved by Bordemann, Meinrenken, and Schlichenmaier [23] using results by Boutet de Monvel and Sjöstrand [26] and Boutet de Monvel and Guillemin [25].

Moreover, by [34, equation (4.1.84), Lemma 7.2.4], as $p \rightarrow+\infty$,

$$
\operatorname{Tr}\left[T_{\mathcal{H}, p}\right]=p^{n} \operatorname{Tr}[\mathcal{H}]+\mathcal{O}\left(p^{n-1}\right) .
$$

Observe that the leading term in (9.122) does not depend on the choice of the metric $g^{T N}$ on $T N$. 
Remark 9.26. Let $\mathcal{T}_{r}$ be the bundle of algebras of Toeplitz operators along the fibres $N$. Over the manifold $M$, this is a bundle of infinite-dimensional algebras. There is no associated underlying bundle of Lie groups. This is why we will have to handle the bundle $\mathcal{T}_{r}$ with some care.

Recall that $\widehat{T X} \simeq T X$. We denote by $\widehat{\omega}\left(\nabla^{F}, g^{F}\right), \widehat{\operatorname{div}}_{N}$ the restrictions of $\omega\left(\nabla^{F}, g^{F}\right), \operatorname{div}_{N}$ to $\widehat{T X}$. If $\widehat{e}_{1}, \ldots, \widehat{e}_{m}$ is an orthonormal basis of $\widehat{T X}$, set

$$
\begin{aligned}
\left|\widehat{\omega}\left(\nabla^{F_{p}}, g^{F_{p}}\right)\right|^{2} & =\sum_{i=1}^{m}\left(\omega\left(\nabla^{F_{p}}, g^{F}\right)\left(\widehat{e}_{i}\right)\right)^{2}, \\
\mid T_{\widehat{\vartheta}}-\widehat{\operatorname{div}}_{N} / 2 p, p & \left.\right|^{2}=\sum_{i=1}^{m} T_{\left.\widehat{\vartheta}-\widehat{\operatorname{div}}_{N} / 2 p\right)\left(\widehat{e}_{i}\right), p}^{2} .
\end{aligned}
$$

Theorem 9.27. The following identities hold:

$$
\begin{aligned}
& \omega\left(\nabla^{F_{p}}, g^{F_{p}}\right) / 2 p=T_{-\vartheta+\operatorname{div}_{N} / 2 p, p}, \\
& \omega\left(\nabla F_{p}, g^{F_{p}}\right)^{2} / 4 p=p T_{\vartheta-\operatorname{div}_{N} / 2 p, p}^{2}, \\
& \frac{1}{4 p^{2}}\left|\widehat{\omega}\left(\nabla^{F_{p}}, g^{F_{p}}\right)\right|^{2}=\left|T_{\widehat{\vartheta}}-\widehat{\operatorname{div}}_{N} / 2 p, p\right|^{2} \text {. }
\end{aligned}
$$

If $\mathcal{H}$ is a smooth section of $\mathcal{A}$, then

$$
\nabla^{F_{p}, u} T_{\mathcal{H}, p}=T_{\left(\nabla \mathcal{A}-2 p \vartheta+\operatorname{div}_{N}\right) \mathcal{H}, p}-\left[T_{-p \vartheta+\operatorname{div}_{N} / 2, p}, T_{\mathcal{H}, p}\right]_{+} .
$$

As $p \rightarrow+\infty$,

$$
\begin{aligned}
& \omega\left(\nabla^{F_{p}}, g^{F_{p}}\right) / 2 p=-T_{\vartheta, p}+\mathcal{O}\left(p^{-1}\right), \\
& \omega\left(\nabla^{F_{p}}, g^{F_{p}}\right)^{2} / 4 p=i T_{\vartheta * 2, p}+\mathcal{O}\left(p^{-1}\right), \\
& \frac{1}{4 p^{2}}\left|\widehat{\omega}\left(\nabla^{F_{p}}, g^{F_{p}}\right)\right|^{2}=T_{|\widehat{\vartheta}|^{2}, p}+\mathcal{O}\left(p^{-1}\right), \\
& \nabla^{F_{p}, u} T_{\mathcal{H}, p}=T_{\nabla \mathcal{A}, u \mathcal{H}, p}+\mathcal{O}\left(p^{-1}\right), \\
& \nabla^{F_{p}} T_{\mathcal{H}, p}=T_{\nabla} \mathcal{A}_{\mathbf{C}^{\prime} \mathcal{H}, p}+\mathcal{O}\left(p^{-1}\right) .
\end{aligned}
$$

Proof. By (9.34), (9.104), and by Theorem 9.25, we get (9.124), (9.125). By (9.18), (9.35), (9.40), (9.121), (9.124), and (9.125), we get the first four equations in (9.126). By (9.104), (9.105), we get

$$
\nabla^{F_{p}} T_{\mathcal{H}, p}=\nabla^{F_{p}, u} T_{\mathcal{H}, p}-p\left[T_{-\vartheta+\operatorname{div}_{N} / 2 p, p}, T_{\mathcal{H}, p}\right] .
$$

By (9.121), we find that

$$
-p\left[T_{-\vartheta+\operatorname{div}_{N} / 2 p, p}, T_{\mathcal{H}, p}\right]=T_{i\{\vartheta, \mathcal{H}\}}+\mathcal{O}\left(p^{-1}\right) .
$$

By (9.53), by the fourth identity in (9.126), by (9.127), and by (9.128), we get the last identity in (9.126). The proof of our theorem is completed. 
Remark 9.28. Note that the leading terms in the right-hand side of (9.126) do not depend on the choice of the metric $g^{T N}$. By (5.3), and by Theorem 9.27, we recover equation (9.44) for $\nabla^{\mathcal{A}, u, 2}$, and the flatness of $\nabla^{\mathcal{A}_{\mathbf{C}},{ }^{\prime}}$.

In the general case of nonflat fibrations, Ma and Zhang [37, Theorem 2.1 and Remark 3.1] have announced that the curvature of the natural unitary connection on $F_{p}$ is a Toeplitz operator in the sense of [34, Chapter 7].

\subsection{The asymptotics of the forms $h\left(\nabla^{F_{p}}, g^{F_{p}}\right)$}

First, we prove an extension of Proposition 6.1.

Proposition 9.29. As $p \rightarrow+\infty$,

$$
p^{-n} \frac{1}{\sqrt{p}} \psi_{1 / \sqrt{p}} h\left(\nabla^{F_{p}}, g^{F_{p}}\right)=\gamma+\mathcal{O}\left(p^{-1}\right) .
$$

Proof. By (5.6), (5.8), we get

$$
\frac{1}{\sqrt{p}} \psi_{1 / \sqrt{p}} h\left(\nabla^{F_{p}}, g^{F_{p}}\right)=(2 i \pi)^{1 / 2} \varphi \operatorname{Tr}\left[\frac{\omega\left(\nabla^{F_{p}}, g^{F_{p}}\right)}{2 p} \exp \left(\omega\left(\nabla^{F_{p}}, g^{F_{p}}\right)^{2} / 4 p\right)\right] \text {. }
$$

Now, we use (9.87), (9.114)-(9.117), (9.121), (9.122), (9.126), and (9.130), and we get $(9.129)$.

\subsection{The spectral gap}

We use the same notation as in $\S 4.2$. By [22, Theorem 4.13], instead of (4.11), we get

$$
\begin{aligned}
D^{X, 2}= & -\Delta^{X, u}+\frac{K^{X}}{4}-\frac{1}{8}\left\langle R^{T X}\left(e_{i}, e_{j}\right) e_{k}, e_{\ell}\right\rangle c\left(e_{i}\right) c\left(e_{j}\right) \widehat{c}\left(e_{k}\right) \widehat{c}\left(e_{\ell}\right) \\
& +\frac{1}{4}\left|\widehat{\omega}\left(\nabla^{F}, g^{F}\right)\right|^{2}-\frac{1}{8}\left(c\left(e_{i}\right) c\left(e_{j}\right)-\widehat{c}\left(e_{i}\right) \widehat{c}\left(e_{j}\right)\right) \omega\left(\nabla^{F}, g^{F}\right)^{2}\left(e_{i}, e_{j}\right) \\
& -\frac{1}{2} c\left(e_{i}\right) \widehat{c}\left(e_{j}\right) \nabla_{e_{i}}^{T X \otimes F, u} \omega\left(\nabla^{F}, g^{F}\right)\left(e_{j}\right) .
\end{aligned}
$$

In (9.131), we replace $F$ by $F_{p}$. Using Theorem 9.27 , and in particular the third identity in (9.126), we deduce from (9.131) that, if $\widehat{\vartheta}$ is nondegenerate, the obvious analogue of Theorem 4.4 still holds.

\subsection{A formula for $L_{t}^{F}$}

Put

$$
\mathcal{R}^{F}=\frac{1}{4}\left\langle e_{i}, R^{T X} e_{j}\right) \widehat{c}\left(e_{i}\right) \widehat{c}\left(e_{j}\right)-\frac{1}{4} \omega\left(\nabla^{F}, g^{F}\right)^{2} .
$$

Set

$$
\begin{aligned}
\Lambda_{t}^{F}= & t \frac{K^{X}}{16}+\frac{t}{8} c\left(e_{i}\right) c\left(e_{j}\right) \mathcal{R}^{F}\left(e_{i}, e_{j}\right)+\frac{1}{2} f^{\alpha} f^{\beta} \mathcal{R}^{F}\left(f_{\alpha}^{H}, f_{\beta}^{H}\right) \\
& +\frac{\sqrt{t}}{2} c\left(e_{i}\right) f^{\alpha} \mathcal{R}^{F}\left(e_{i}, f_{\alpha}^{H}\right)+\frac{t}{16}\left|\widehat{\omega}\left(\nabla^{F}, g^{F}\right)\right|^{2}-\frac{\sqrt{t}}{4} f^{\alpha} \widehat{c}\left(e_{i}\right) \nabla_{f_{\alpha}^{H}}^{T X \otimes_{\mathbf{R}} F, u} \omega\left(\nabla^{F}, g^{F}\right)\left(e_{i}\right)
\end{aligned}
$$




$$
\begin{aligned}
& +\frac{t}{32} \widehat{c}\left(e_{i}\right) \widehat{c}\left(e_{j}\right) \omega\left(\nabla^{F}, g^{F}\right)^{2}\left(e_{i}, e_{j}\right)-\frac{t}{8} c\left(e_{i}\right) \widehat{c}\left(e_{j}\right) \nabla_{e_{i}}^{T X \otimes_{\mathbf{R}} F, u} \omega\left(\nabla^{F}, g^{F}\right)\left(e_{j}\right) \\
& -\frac{1}{4} z \sqrt{t} c\left(e_{i}\right) \omega\left(\nabla^{F}, g^{F}\right)\left(e_{i}\right)-\frac{1}{2} z f^{\alpha} \omega\left(\nabla^{F}, g^{F}\right)\left(f_{\alpha}^{H}\right) .
\end{aligned}
$$

By [18, Theorem 3.11], [15, Theorem 3.19], instead of (5.67), we get

$$
L_{t}^{F}=-\frac{t}{4}\left({ }^{1} \nabla_{t / 2, e_{i}}^{\pi^{*} \Lambda^{\cdot}\left(T^{*} S\right) \widehat{\otimes} \Lambda^{\cdot}\left(T^{*} X\right) \otimes_{\mathbf{R}} F, u}-\frac{z}{\sqrt{t}} \widehat{c}\left(e_{i}\right)\right)^{2}+\Lambda_{t}^{F} .
$$

\subsection{An extension of Theorem 6.5}

Now, we extend Theorem 6.5.

Theorem 9.30. As $p \rightarrow+\infty$,

$$
\begin{aligned}
& \frac{1}{\sqrt{p}} \psi_{1 / \sqrt{p}} h\left(A^{\prime}, g_{t / p^{2}}^{\Omega^{2}\left(X,\left.F_{p}\right|_{X}\right)}\right)=\pi_{*} c_{t / 4} p^{n}+\mathcal{O}\left(p^{n-1}\right), \\
& \psi_{1 / \sqrt{p}} \frac{1}{\sqrt{t}} h^{\wedge}\left(A^{\prime}, g_{t / p^{2}}^{\Omega^{\cdot}\left(X, F_{p} \mid X\right)}\right)=\pi_{*} \frac{1}{\sqrt{t}} d_{t / 4} p^{n+1}+\mathcal{O}\left(p^{n}\right) .
\end{aligned}
$$

Moreover, the obvious analogue of (6.24) still holds. If $\widehat{\vartheta}$ is nondegenerate, the analogue of (6.25) also holds.

Proof. As in the proof of Theorem 6.5, we may and we will assume $M$ to be compact.

Let $\mathcal{H}$ be a smooth section of $\mathcal{A}$. By (9.117), (9.126), there exists $C>0$ such that, for $p \in \mathbf{N}$,

$$
\left\|\nabla^{F_{p}, u} T_{\mathcal{H}, p}\right\| \leqslant C .
$$

We use the same notation as in $\S 6.4$. As in (6.17), we get

$$
h\left(A^{\prime}, g_{t}^{\Omega \cdot\left(X,\left.F\right|_{X}\right)}\right)=(2 i \pi)^{1 / 2} \varphi \int_{X} \operatorname{Tr}_{\mathrm{s}}\left[P_{t}^{F}(x, x)\right]^{z} d v_{X}(x) .
$$

Put

$$
\Lambda_{p, t}=\theta_{1 / \sqrt{p}} \Lambda_{4 t / p^{2}}^{F_{p}} \theta_{\sqrt{p}} .
$$

By (9.134), as in (6.30), we get

$$
\theta_{1 / \sqrt{p}} L_{4 t / p^{2}}^{F_{p}} \theta_{\sqrt{p}}=-\frac{t}{p^{2}}\left({ }^{1} \nabla_{2 t / p, e_{i}}^{\pi^{*} \Lambda^{\cdot}\left(T^{*} S\right) \widehat{\otimes} \Lambda^{\cdot}\left(T^{*} X\right) \otimes_{\mathbf{R}} F_{p}, u}-\frac{z}{2 \sqrt{t}} \sqrt{p} \widehat{c}\left(e_{i}\right)\right)^{2}+\Lambda_{p, t} .
$$

Let $x \in M$, and let $X$ be the fibre of $\pi$ containing $x$. We use the same coordinate system near $x$ as in the proof of Theorem 6.5, and we use the same trivialization of $\mathbf{R}[z] \widehat{\otimes} \Lambda^{*}\left(T^{*} S\right) \widehat{\otimes} \Lambda^{*}\left(T^{*} X\right)$. For $p \in \mathbf{N}$, we trivialize the vector bundle $F_{p}$ on $B(x, \epsilon)$ using parallel transport for the unitary connection $\nabla^{F_{p}, u}$ along the geodesics centred at $x$, so that the identification preserves the Hermitian metric of $F_{p}$.

For $Y \in T_{x} X,|Y| \leqslant \epsilon$, let $\mathcal{H}_{Y} \in \mathcal{A}_{Y}$ be the restriction of $\mathcal{H}$ to the fibre $N_{Y}$. Then $T_{\mathcal{H}_{Y}, p}$ acts on $F_{p, Y} \simeq F_{p, x}$. By the crucial equation (9.136), for $Y \in T_{x} X,|Y| \leqslant \epsilon$, we get

$$
\left\|T_{\mathcal{H}_{Y}, p}-T_{\mathcal{H}_{0}, p}\right\| \leqslant C|Y| .
$$


If $f \in C^{\infty}\left(T_{x} X \times N_{x}, L^{p}\right)$, set

$$
K_{s}(f(Y, z))=f(s Y, z)
$$

Put

$$
M_{p, t}^{F_{p}}=K_{\sqrt{t} / p} \theta_{1 / \sqrt{p}} L_{4 t / p^{2}}^{F_{p}} \theta_{\sqrt{p}} K_{p / \sqrt{t}} .
$$

We make the same Getzler rescaling on the Clifford variables $c(e), \widehat{c}(e)$ as in the proof of Theorem 6.5. Let $N_{p, t}^{F_{p}}$ be obtained from $M_{p, t}^{F_{p}}$ by this rescaling.

Since the vector spaces $C^{\infty}\left(N_{x}, L^{p}\right)$ depend on $p$, as $p \rightarrow+\infty$, it is not possible to give an asymptotic expansion for the operators $N_{p, t}^{F_{p}}$ in a naive sense. However, since the coefficients of these operators lie in the Toeplitz algebras $\mathcal{T}_{\sqrt{t} Y / p}$, the asymptotics will be ultimately described in terms of the Toeplitz algebra $\mathcal{T}_{x}$.

Let $\mathrm{Op}_{x}$ be the algebra of scalar differential operators on $T_{x} X$. Let $\mathcal{B}_{x}$ be the algebra

$$
\mathcal{B}_{x}=\mathrm{Op}_{x} \otimes \mathbf{R}[z] \widehat{\otimes} \Lambda^{*}\left(T^{*} S\right) \widehat{\otimes} \operatorname{End}\left(\Lambda^{*}\left(T^{*} X\right)\right) \widehat{\otimes} \operatorname{End}\left(\Lambda^{\cdot}\left(\widehat{T^{*} X}\right)\right) \otimes \mathcal{T}_{x} .
$$

Our computations will ultimately take place in the algebra $\mathcal{B}_{x}$.

Let $\mathcal{R}_{p, t}^{F_{p}}, \Lambda_{p, t}^{F_{p}}$ be obtained from $\mathcal{R}^{F_{p}}, \Lambda_{4 t / p^{2}}^{F_{p}}$ by the above conjugations, trivializations, and Getzler rescalings. By (9.132), we get

$$
\mathcal{R}_{p, t}^{F_{p}}=\frac{1}{4}\left\langle e_{i}, R_{\sqrt{t} Y / p}^{T X} e_{j}\right| \widehat{c}_{1 / p}\left(e_{i}\right) \widehat{c}_{1 / p}\left(e_{j}\right)-\frac{1}{4} \omega\left(\nabla^{F_{p}}, g^{F_{p}}\right)_{\sqrt{t} Y / p}^{2} .
$$

By (9.133), when evaluating the tensors at $\sqrt{t} Y / p$, we get

$$
\begin{aligned}
\Lambda_{p, t}^{F_{p}}= & 4 t \frac{K^{X}}{16 p^{2}}+\frac{t}{2 p^{2}} c_{t / p}\left(e_{i}\right) c_{t / p}\left(e_{j}\right) \mathcal{R}_{p, t}^{F_{p}}\left(e_{i}, e_{j}\right)+\frac{1}{2 p} f^{\alpha} f^{\beta} \mathcal{R}_{p, t}^{F_{p}}\left(f_{\alpha}^{H}, f_{\beta}^{H}\right) \\
& +\frac{\sqrt{t}}{p^{3 / 2}} c_{t / p}\left(e_{i}\right) f^{\alpha} \mathcal{R}_{p, t}^{F_{p}}\left(e_{i}, f_{\alpha}^{H}\right)+\frac{t}{4 p^{2}}\left|\widehat{\omega}\left(\nabla^{F_{p}}, g^{F_{p}}\right)\right|^{2} \\
& -\frac{\sqrt{t}}{2 p^{3 / 2}} f^{\alpha} \widehat{c}_{1 / p}\left(e_{i}\right) \nabla_{f_{\alpha}^{H}}^{T X \otimes_{\mathbf{R}} F_{p}, u} \omega\left(\nabla^{F_{p}}, g^{F_{p}}\right)\left(e_{i}\right) \\
& +\frac{t}{8 p^{2}} \widehat{c}_{1 / p}\left(e_{i}\right) \widehat{c}_{1 / p}\left(e_{j}\right) \omega\left(\nabla^{F}, g^{F}\right)^{2}\left(e_{i}, e_{j}\right) \\
& -\frac{t}{2 p^{2}} c_{t / p}\left(e_{i}\right) \widehat{c}_{1 / p}\left(e_{j}\right) \nabla_{e_{i}}^{T X \otimes F_{p}, u} \omega\left(\nabla^{F_{p}}, g^{F_{p}}\right)\left(e_{j}\right) \\
& -\frac{1}{2 p^{3 / 2}} z \sqrt{t} c_{t / p}\left(e_{i}\right) \omega\left(\nabla^{F_{p}}, g^{F_{p}}\right)\left(e_{i}\right)-\frac{1}{2 p} z f^{\alpha} \omega\left(\nabla^{F_{p}}, g^{F_{p}}\right)\left(f_{\alpha}^{H}\right) .
\end{aligned}
$$

In what follows, the tensors will be evaluated at $x$. We use the same conventions as in $\S 9.8$. By (9.34), by Theorem 9.27, and by (9.140)-(9.145), as $p \rightarrow+\infty$,

$$
\begin{aligned}
\Lambda_{p, t}^{F_{p}}= & \frac{1}{4}\left\langle e_{i},\left.R^{T X} e_{j}\left|\widehat{e}^{i} \widehat{e}^{j}+T_{-i \vartheta * 2}+\sqrt{t} \nabla \widehat{T X} \otimes \mathcal{A}, u \widehat{\vartheta}+t\right| \widehat{\vartheta}\right|^{2}+i t \widehat{\vartheta}{ }^{* 2}+z \vartheta, p\right. \\
& +(1+\sqrt{t}|Y|) \mathcal{O}\left(p^{-1}\right) .
\end{aligned}
$$

Incidentally, observe that, by using higher-order versions of the fourth equation in (9.126) and taking a Taylor expansion, one verifies easily that $\Lambda_{p, t}^{F_{p}}$ lies in the Toeplitz algebra $\mathcal{T}_{x}$, so that (9.146) gives its expansion to order 0. 
Let $E_{p, t}^{F_{p}}$ be obtained from the first term in the right-hand side of (9.139) by conjugation by $K_{\sqrt{t} / p}$ and by doing the above Getzler rescalings. We claim that, as $p \rightarrow+\infty$,

$$
E_{p, t}^{F_{p}}=-\Delta^{T X}+|Y| \mathcal{O}\left(p^{-1}\right),
$$

the last term in the right-hand side of (9.147) containing differentiation up to order 2 on $T_{x} X$, the uniform bound $\mathcal{O}\left(p^{-1}\right)$ only referring to the part acting on $F_{p, x}$. To obtain (9.147), we use in particular (5.3) and the second identity in (9.126).

Set

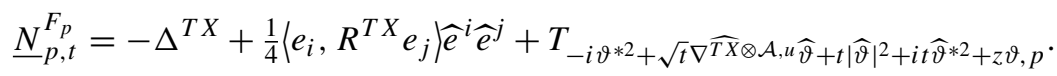

By (9.139), (9.146), and (9.147), as $p \rightarrow+\infty$,

$$
N_{p, t}^{F_{p}}=\underline{N}_{p, t}^{F_{p}}+(1+\sqrt{t}|Y|) \mathcal{O}\left(p^{-1}\right) .
$$

Equation (9.149) can be viewed as the expansion of $N_{p, t}^{F_{p}} \in \mathcal{B}_{x}$, with the conventions of Ma and Marinescu in [34, Chapter 7].

Set

$$
O_{p, t}^{F_{p}}=i^{-\mathcal{N}^{d} / 2} \underline{N}_{p, t}^{F_{p}} i^{\mathcal{N}^{d} / 2}
$$

By (9.72), (9.148), we get

$$
O_{p, t}^{F_{p}}=-\Delta^{T X}+T_{\sigma\left(\mathcal{A}_{t}^{2}\right)+\frac{z}{\sqrt{i}} \theta, p} .
$$

Also,

$$
\exp \left(-O_{p, t}^{F_{p}}\right)=\exp \left(\Delta^{T X}\right) \exp \left(-T_{\sigma\left(\mathcal{A}_{t}^{2}\right)+\frac{z}{\sqrt{i}} \theta, p}\right) .
$$

Let $\exp \left(-O_{p, t}^{F_{p}}\right)\left(Y, Y^{\prime}\right)$ be the smooth kernel of the operator $\exp \left(-O_{p, t}^{F_{p}}\right)$ with respect to the volume form $d Y^{\prime}$. By (9.152), we deduce that

$$
\operatorname{Tr}^{F_{p}}\left[\int^{\widehat{B}} \exp \left(-O_{p, t}^{F_{p}}\right)(0,0)\right]=(4 \pi)^{-m / 2} \operatorname{Tr}^{F_{p}}\left[\int^{\widehat{B}} \exp \left(-T_{\sigma\left(\mathcal{A}_{t}^{2}\right)+\frac{z}{\sqrt{i}} \theta, p}\right)\right] .
$$

Given $p \in \mathbf{N}$, we have the identity of operators acting on $F_{p, x}$,

$$
\exp \left(-T_{\sigma\left(\mathcal{A}_{t}^{2}\right)+\frac{z}{\sqrt{i}} \vartheta, p}\right)=\sum_{k=0}^{+\infty} \frac{\left(-T_{\sigma\left(\mathcal{A}_{t}^{2}\right)+\frac{z}{\sqrt{i}} \vartheta, p}\right)^{k}}{k !}
$$

By (9.117), (9.120), there exists $C>0$ such that, for $k \in \mathbf{N}, p \in \mathbf{N}^{*}$,

$$
\left\|\left(-T_{\sigma\left(\mathcal{A}_{t}^{2}\right)+\frac{z}{\sqrt{i}} \vartheta, p}\right)^{k}-T_{\left(-\sigma\left(\mathcal{A}_{t}^{2}\right)-\frac{z}{\sqrt{i}} \vartheta\right)^{k}, p}\right\| \leqslant C \frac{k}{p} .
$$

By (9.154), (9.155), we find that

$$
\left\|\exp \left(-T_{\sigma\left(\mathcal{A}_{t}^{2}\right)+\frac{z}{\sqrt{i}} \vartheta, p}\right)-T_{\exp \left(-\sigma\left(\mathcal{A}_{t}^{2}\right)-\frac{z}{\sqrt{i}} \vartheta\right), p}\right\| \leqslant \frac{C}{p} .
$$


By (9.114), (9.156), we get

$$
p^{-n}\left\|\exp \left(-T_{\sigma\left(\mathcal{A}_{t}^{2}\right)+\frac{z}{\sqrt{i}} \vartheta, p}\right)-T_{\exp \left(-\sigma\left(\mathcal{A}_{t}^{2}\right)-\frac{z}{\sqrt{i}} \vartheta\right), p}\right\|_{1} \leqslant \frac{C}{p} .
$$

Using (9.122), we get

$$
\begin{aligned}
& p^{-n} \operatorname{Tr}^{F_{p}}\left[\int^{\widehat{B}} T_{\left.\exp \left(-\sigma\left(\mathcal{A}_{t}^{2}\right)-\frac{z}{\sqrt{i}} \vartheta\right), p\right]}\right. \\
& \quad=\operatorname{Tr}\left[\int^{\widehat{B}} \exp \left(-\sigma\left(\mathcal{A}_{t}^{2}\right)-\frac{z}{\sqrt{i}} \vartheta\right)\right]+\mathcal{O}\left(p^{-1}\right) .
\end{aligned}
$$

By (6.56), (9.89), (9.149), (9.153), and (9.158), as long as the proper localization and limit arguments are established, we get the first identity in (9.135). In what follows, we concentrate on the proof of these arguments.

First, we prove that the asymptotics of the integral in the right-hand side of (9.137) can be localized near any $x \in X$. By (9.120), by Theorem 9.27, and by (9.132), (9.133), given $t>0$, there is $C>0$ such that, for $p \in \mathbf{N}^{*}$,

$$
\left\|\theta_{1 / \sqrt{p}} \Lambda_{4 t / p^{2}}^{F_{p}} \theta_{\sqrt{p}}\right\| \leqslant C .
$$

Let $p_{t}\left(x, x^{\prime}\right)$ be the smooth kernel associated with the scalar heat operator $\exp \left(t \Delta^{X}\right)$. Using Kato's domination principle and (9.159), there exists $k \in \mathbf{N}$ such that, for $p \in \mathbf{N}^{*}$,

$$
\left\|P_{4 t / p^{2}}^{F_{p}}\left(x, x^{\prime}\right)\right\| \leqslant C p_{4 t / p^{2}}\left(x, x^{\prime}\right) p^{k} .
$$

By (9.114), (9.160), we get

$$
\left\|P_{4 t / p^{2}}^{F_{p}}\left(x, x^{\prime}\right)\right\|_{1} \leqslant C p_{4 t / p^{2}}\left(x, x^{\prime}\right) p^{n+k} .
$$

Also, there are constants $c>0, C>0$ such that, for $0<t \leqslant 1, p \in \mathbf{N}$,

$$
p_{t / 4 p^{2}}\left(x, x^{\prime}\right) \leqslant C\left(p^{2} / t\right)^{m / 2} \exp \left(-c p^{2} d^{2}\left(x, x^{\prime}\right) / t\right) .
$$

By (9.160)-(9.162), we get

$$
\left\|P_{4 t / p^{2}}^{F_{p}}\left(x, x^{\prime}\right)\right\|_{1} \leqslant C p^{m+n+k} t^{-m / 2} \exp \left(-c p^{2} d^{2}\left(x, x^{\prime}\right) / t\right) .
$$

By (9.163), we deduce easily that, given $x \in X$, the integral in (9.137) can be localized near $x \in X$. As in the proof of Theorem 6.5, this means that, to compute the asymptotics as $p \rightarrow+\infty$ of (9.137), we may as well fix $x \in X$, and replace near $x$ the fibre $X$ by $T_{X} X$. Similarly, we may as well assume the projection $q:\left.\mathcal{N}\right|_{X} \rightarrow X$ maps to $T_{x} X$. We will still denote by $P_{t}^{F_{p}}\left(Y, Y^{\prime}\right), Y, Y^{\prime} \in T_{x} X$ the heat kernel associated with the operator $\exp \left(-L_{t}^{F_{p}}\right)$. We make exactly the same change of coordinates as in (9.142) and the same rescalings as before. The major difference is that the coordinates are now taken in $T_{x} X$, so that they are globally defined on $T_{x} X$. 
For $s>0$, let $Q_{p, t, s}^{F_{p}}\left(Y, Y^{\prime}\right), \underline{Q}_{p, t, s}^{F_{p}}\left(Y, Y^{\prime}\right), Y, Y^{\prime} \in T_{x} X$ be the smooth kernels associated with the operators $\exp \left(-s N_{p, t}^{F_{p}}\right), \exp \left(-s \underline{N}_{p, t}^{F_{p}}\right)$ with respect to the volume $d v_{T X}\left(Y^{\prime}\right)$. We claim that, for $s>0$, as $p \rightarrow+\infty$,

$$
\left\|\left(Q_{p, t, s}^{F_{p}}-\underline{Q}_{p, t, s}^{F_{p}}\right)\left(Y, Y^{\prime}\right)\right\|=\mathcal{O}\left(p^{-1}\right),
$$

and that, in (9.164), $\mathcal{O}\left(p^{-1}\right)$ is uniform for bounded $Y, Y^{\prime}$. If $F_{p}$ was not depending on $p$, so that the kernels in (9.164) act on a fixed vector space $F_{x},(9.164)$ would just follow from simple uniform estimates on the kernels, from (9.149), and from Duhamel's formula. The main difficulty here is that $F_{p}$ changes with $p$. However, the estimates in (9.146), (9.147) that are uniform in $p \in \mathbf{N}$ are enough to establish (9.164).

By (9.164), we get

$$
\left\|\left(Q_{p, t, s}^{F_{p}}-\underline{Q}_{p, t, s}^{F_{p}}\right)(0,0)\right\|=\mathcal{O}\left(p^{-1}\right) .
$$

Recall that the functional $\widehat{\operatorname{Tr}}_{\mathrm{s}}$ was defined after (6.56). We extend $\widehat{\operatorname{Tr}}_{\mathrm{s}}$ to a map $\widehat{\operatorname{Tr}}_{\mathrm{s}} F_{p}=$ $\widehat{\operatorname{Tr}}_{\mathrm{s}} \otimes \operatorname{Tr}^{F}$. By (9.114), (9.165), we find that

$$
p^{-n} \widehat{\operatorname{Tr}}_{\mathrm{s}}^{F_{p}}\left[\left(Q_{p, t, s}^{F_{p}}-\underline{Q}_{p, t, s}^{F_{p}}\right)(0,0)\right]=\mathcal{O}\left(p^{-1}\right) .
$$

By proceeding as in the proof of Theorem 6.5, and using (9.89) and the above arguments, we get the first identity in (9.135). As in the proof of Theorem 6.5, the second identity in (9.135) is a consequence of the first one.

The arguments that are needed to establish the analogue of equations (6.24) and (6.25) are essentially the same as in the proof of Theorem 6.5, once the above convergence results are taken into account. The proof of our theorem is completed.

Remark 9.31. Let $\widehat{\pi}: \widehat{X} \rightarrow X$ be a Galois cover of $X$ as in $\S 6.6$. Then the obvious analogue of the results of that subsection still hold. The arguments needed in the proof are the ones that are used in $\S 6.6$ as well as in the proof of Theorem 9.30.

\subsection{The asymptotics of the analytic torsion forms}

We still assume $\widehat{\vartheta}$ to be nondegenerate. Now, we establish the obvious extension of Theorem 7.1.

Theorem 9.32. As $p \rightarrow+\infty$,

$$
p^{-n-1} \psi_{1 / \sqrt{p}} \mathcal{T}_{h}\left(T^{H} M, g^{T X}, \nabla^{F_{p}}, g^{F_{p}}\right)=\pi_{*} W+\mathcal{O}\left(p^{-1}\right) .
$$

Proof. Using Theorem 9.30 instead of Theorem 6.5, the proof of our theorem is essentially the same as the proof of Theorem 7.1. Let us now give some extra details.

We still have to establish the two identities in equation (7.4). The proof of the first identity is exactly the same as in $\S 7.2$, using the analogue of Theorem 4.4 that was established in $\S 9.10$. As to the proof of the second identity, we still write equation (7.13). By Theorem 9.30, equation (7.14) still holds. Once the analogue of (7.15) is established, by Theorem 9.30 , we get the analogue of (7.16), which concludes the proof of our theorem. 
The estimate (7.15) can be proved exactly as in $\S 7.3$. This completes the proof of our theorem.

Remark 9.33. The extensions of the results obtained in Remark 7.3 and in $\S \S 7.5$ and 7.6 still hold. Indeed, by Ma and Marinescu [36, Theorem 0.3 and Remark 0.5], when $L^{\prime}$ is a holomorphic vector bundle on $N$, when replacing $L^{p}$ by $L^{p} \otimes L^{\prime}$, the obvious analogue of equation (9.120) holds. Moreover, by [34, equation (4.1.84), Lemma 7.2.4], equation (9.122) still holds, when multiplying the right-hand side by $\operatorname{dim} L^{\prime}$.

\subsection{The case of reductive groups}

The case that was considered in $\S \S 2,6$, and 7 is a special case of what was done in the present section. Indeed, under the assumptions of the above subsections, the line bundle $L$ on $\mathcal{N}$ is canonically equipped with a metric $g^{L}$. Then, if $c_{1}\left(L, g^{L}\right)$ is the corresponding first Chern form of $L$ that is defined as in (9.10), using the notation of $\S \S 1$ and 2 , we get

$$
\kappa=c_{1}\left(L, g^{L}\right) .
$$

If $A \in \mathfrak{u}$, the vector field $A^{N}$ on $N$ is the Hamiltonian vector field associated with the Hamiltonian $2 \pi\langle\mu, A\rangle$ in the sense of Definition 9.1. By (1.21), (9.30), if $A, B \in \mathfrak{u}$,

$$
\{\langle 2 \pi \mu, A\rangle, 2 \pi\langle\mu, B\rangle\}=-2 \pi\langle\mu,[A, B]\rangle .
$$

The - sign in (9.169) is related to the fact that $A \rightarrow-A^{N}$ is a morphism of Lie algebras.

We have the identity

$$
\vartheta=2 \pi\left\langle\mu, i \theta^{\mathfrak{p}}\right\rangle
$$

By (9.169), (9.170), we get

$$
\vartheta^{* 2}=2 \pi\left\langle\mu, \theta^{\mathfrak{p}, 2}\right\rangle
$$

By (9.170), we obtain

$$
\widehat{\vartheta}=2 \pi\left\langle\mu, i \widehat{\theta}^{\mathfrak{p}}\right\rangle
$$

By (1.25), (9.172), we find that

$$
\sigma_{\widehat{\theta} \mathfrak{p}}=\sigma_{\widehat{\vartheta}} .
$$

From (9.173), we deduce that $\widehat{\theta}^{\mathfrak{p}}$ is nondegenerate in the sense of Definition 1.1 if and only if $\widehat{\vartheta}$ is nondegenerate in the sense of Definition 9.13.

By comparing Theorems 2.10 and 9.21, and also 2.12 and 9.23, and using (9.170), it is clear that the forms that were obtained in $\S 2$ are special cases of the forms obtained in the present section.

\section{Index}

$\|A\|_{1}, 334$

$\|A\|, 334$

$\mathcal{A}, 324$

$\mathscr{A}, 234$
$\mathcal{A}_{\mathbf{C}}, 326$

$A^{N}, 234$

analytic torsion forms, 275

$A_{t}, 241$ 
$\mathcal{A}_{t}, 238$

$\underline{a}_{t}, 241,253,328,331$

$a_{t}, 243,328$

$\mathscr{A}^{U}, 234$

$B, 231$

$\mathfrak{b}, 307$

Berezin integral, 235

$\underline{b}_{t}, 241,253,328,331$

$b_{t}, 243,328$

$c_{1}^{V}\left(L, g^{L}\right), 322$

$\widehat{c}(\widehat{E}), 232$

$\widehat{c}(\widehat{e}), 233$

$c(\widehat{E}), 232$

$c(\widehat{e}), 233$

$\chi(X, F), 273$

$C^{\mathfrak{g}}, 301$

$C^{\mathfrak{g}, E}, 302$

$C^{\mathfrak{g}, H}, 301$

$C^{\mathfrak{g}, H, E}, 302$

$C^{\mathfrak{g}, X}, 304$

$C^{\mathfrak{k}}, 301$

$C^{\mathfrak{k}, E}, 302$

$c_{1}\left(L, g^{L}\right), 261,322$

$\chi_{p}, 264$

$\widehat{c}_{s}(\widehat{e}), 239$

$\widehat{c}_{s}(e), 285$

$c_{s}(e), 285$

$c_{t}, 253,283,331$

$C_{t}^{\mathscr{F}}, 280$

$\chi(X), 251$

$\delta_{a}, 239$

$d^{G_{\mathbf{C}}}, 287$

$\operatorname{div}_{N}(U), 321$ $d_{t}, 253,283,331$

$D_{t}^{\mathscr{F}}, 280$

$d v_{N}, 325$

$d v_{X}, 248,267$

$D^{X}, 267$

$\underline{D}^{X}, 304$

$d^{X}, 267$

$\Delta^{X, u}, 267$

$e\left(\widehat{E}, \nabla^{\widehat{E}}\right), 237$

$e_{t}, 253,331$

$\eta, 234,260$

$F, 266,333$

F, 280

$\mathcal{F}, 333$

$\mathscr{F}, 279$

$\varphi, 242,273,307$

$\underline{\varphi}, 328$

$F_{p}, 268$

G, 231

$\gamma, 252,331$

$\mathfrak{g}, 231$

$G_{\mathbf{C}}, 232$

$\mathfrak{g C}_{\mathbf{C}}, 232$

$\widetilde{\gamma}\left(h, h^{\prime}\right), 253$

$G_{r}, 236$

$\mathfrak{g}_{r}, 236$

$g_{t}^{T X}, 272$

$\|\mathcal{H}\|_{\infty}, 334$

$\mathfrak{h}, 307$

$h\left(A^{\prime}, g_{t}^{\Omega \cdot(X, F \mid X)}\right), 273$

$\mathscr{H}\left(G_{\mathbf{C}}\right), 265,279$

$\left\{\mathcal{H}, \mathcal{H}^{\prime}\right\}, 324$

$\mathscr{H}(N), 234$ 
$h^{\wedge}\left(A^{\prime}, g_{t}^{\Omega \cdot\left(X,\left.F\right|_{X}\right)}\right), 274$

$H \cdot\left(X,\left.F\right|_{X}\right), 267$

$\int^{\widehat{B}}, 235$

$J_{1}\left(Y_{0}^{\mathfrak{k}}\right), 305$

K, 231

$\kappa, 243,343$

$\mathfrak{k}, 231$

$\kappa^{\mathfrak{k}}, 302$

$\kappa^{\mathfrak{p}}, 302$

$K_{s, a}, 285$

$K^{X}, 267,277$

L, 253, 310, 331

$\Lambda, 278$

$\mathrm{L}_{A}^{L}, 261$

$\Lambda_{t}^{F}, 337$

$L^{F_{p}}, 293$

$\Lambda_{p, t}, 284$

$L_{t}, 277$

$\mathcal{L}_{t}, 240,248,327,329$

$\widehat{\mathcal{L}}_{t}, 250,329$

$L_{t}^{\mathscr{F}}, 280$

$L_{t}^{F}, 278$

$L_{U^{H}}, 247$

$\mathcal{L}^{X, F}, 304$

m, 246, 300

$m^{\prime}, 300$

$M_{p, t}^{\mathscr{F}}, 285$

$\mu, 234,260$

$\mathcal{N}, 243,321$

$\nabla^{\mathcal{A}_{\mathbf{C}},{ }^{\prime}, 326}$

$\nabla^{\mathcal{A}_{\mathbf{C}}, u}, 326$
$\nabla^{\mathcal{A}}, 325$

$\nabla^{T X}, 247$

$\nabla^{\mathcal{A}, u}, 325$

$\mathcal{N}^{d}, 250,329$

$\nabla^{F}, 333$

$\nabla^{\mathcal{F}, u}, 333$

$\nabla^{\mathcal{F}}, 333$

$\nabla^{\mathscr{F}}, 280$

$\nabla^{F, f}, 300$

$N_{p, t}^{\mathscr{F}}, 285$

$N_{\infty, t}^{\mathscr{F}}, 285$

$\nabla^{\mathbf{F}, u}, 280$

$\nabla^{\mathscr{F}}, u, 280$

$\nabla^{\mathfrak{g}_{r}, u}, 237$

$N^{\Lambda \cdot\left(T^{*} M\right)}, 250$

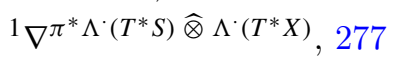

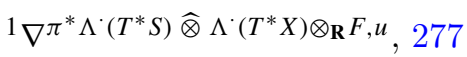

${ }^{1} \nabla_{t}^{\pi^{*} \Lambda^{\cdot}\left(T^{*} S\right) \widehat{\otimes} \Lambda^{\cdot}\left(T^{*} X\right) \otimes_{\mathbf{R}} F, u}, 277$

$N^{\Lambda \cdot\left(\widehat{T^{*} X}\right)}, 250$

$\nabla^{\Lambda \cdot\left(T^{*} X\right) \widehat{\otimes}_{\mathbf{R}} F, u}, 271$

$\nabla^{L, u}, 321$

$\nabla^{\Omega \cdot\left(X,\left.F\right|_{X}\right)}, 271$

$\omega_{A}, 235$

$o(\widehat{E}), 235,241$

$\mathcal{O}\left(e^{-c_{K} t}\right), 244$

$\omega\left(\nabla^{F}, g^{F}\right), 267$

$\omega\left(\nabla^{\mathcal{F}}, g^{\mathcal{F}}\right), 333$

$\omega\left(L, g^{L}\right), 321$

$\mathcal{O}\left(p^{k}\right), 279$

Op, 280

$o(T X), 255$

$\Omega \cdot\left(X,\left.F\right|_{X}\right), 267$

P, 262

$\mathfrak{p}, 231$ 
Pf, 236

$P_{G}^{\prime}, 280$

$\mathcal{P}_{G_{\mathbf{C}}}, 279$

$\psi, 242,328$

$\psi_{a}, 239,272$

$P^{T X}, 246$

$P_{t}\left(x, x^{\prime}\right), 305$

Q, 280

$\mathcal{R}, 277$

$\mathcal{R}^{F}, 337$

$\underline{\mathcal{R}}, 284$

$R(A), 234$

$R_{a, b}(A), 313$

$R^{F, u}, 267$

$R^{\mathscr{F}}, u, 280$

$r^{L}, 261,321$

$r^{L, u}, 321$

$\rho_{p}, 263$

$R^{T X}, 248$

S, 247

$\mathcal{S}, 236,323$

$\mathcal{S}^{\prime}, 248$

$\sigma, 232,233$

$s, 322$

$\sigma\left(\mathcal{A}_{t}^{2}\right), 238,327,329$

$\sigma_{\beta}, 235,243,327$

$S \mathfrak{g}, 232$

$\sigma_{\widehat{\theta}} \mathfrak{p}, 253$

$T, 247,307$

$\Theta, 268$

$\mathcal{T}, 323$

t, 307

$T^{0}, 249,329$

$\theta_{a}, 277$

$\theta^{\mathfrak{g}}, 237,300$
$T^{H}, 246$

$\widehat{T}^{H}, 249$

$\mathcal{T}_{h}\left(T^{H} M, g^{T X}, \nabla^{F}, g^{F}\right), 275$

$\vartheta^{H}, 329$

$\Theta, 231$

$\mathcal{T}_{h}^{\Gamma}\left(g^{T X}, \nabla^{F_{p}}, g^{F_{p}}\right), 290$

$\Theta^{\mathfrak{k}}, 237$

$T^{H} M, 246$

$T^{H} \mathcal{N}, 322$

$T_{0}^{H} \mathcal{N}, 321$

$T_{\mathcal{H}, p}, 335$

$\Theta^{\mathfrak{k}}, 300$

$\theta^{\mathfrak{k}}, 237,300$

$\widehat{\theta}^{\mathfrak{p}}, 248$

$\theta^{\mathfrak{p}}, 237,300$

$\mathcal{T}_{r}, 336$

$\operatorname{Tr}^{\Gamma}[Q], 290$

$\operatorname{Tr}[\mathcal{H}], 325$

$\operatorname{Tr}_{\mathrm{s}}, 230$

$\operatorname{Tr}_{\mathrm{s}}{ }^{[1]}\left[\exp \left(-t D^{X, 2} / 2\right)\right], 305$

$\widehat{\operatorname{Tr}}_{\mathrm{s}}, 288,342$

$\vartheta(s), 276$

$\vartheta^{X}, 329$

$U, 232$

$\mathfrak{u}, 232$

$U \mathfrak{g}, 232$

$U^{H}, 246,322$

$U_{\mathbf{R}} \mathfrak{g}_{\mathbf{C}}, 280$

V, 324

$\operatorname{Vol}(N), 325$

W, 255, 332

$W_{a, b}, 313$

$\widehat{\mathcal{X}}, 253,331$

$X_{\mathcal{H}}, 323$

$\xi, 322$ 


\section{References}

1. M. F. Атіуан, Elliptic operators, discrete groups and von Neumann algebras, in Colloque 'Analyse et Topologie' en l'Honneur de Henri Cartan (Orsay, 1974), Astérisque, 32-33, pp. 43-72 (Soc. Math. France, Paris, 1976), MR 0420729 (54 \#8741).

2. F. A. Berezin, Quantization, Izv. Akad. Nauk SSSR Ser. Mat. 38 (1974), 1116-1175, MR 0395610 (52 \#16404).

3. N. Bergeron and A. Venkatesh, The asymptotic growth of torsion homology for arithmetic groups, J. Inst. Math. Jussieu 12(2) (2013), 391-447, MR 3028790.

4. N. Berline, E. Getzler and M. Vergne, Heat kernels and Dirac operators, in Grundl. Math. Wiss. Band 298 (Springer, Berlin, 1992), MR 94e:58130.

5. N. Berline And M. Vergne, Zéros d'un champ de vecteurs et classes caractéristiques équivariantes, Duke Math. J. 50(2) (1983), 539-549, MR 84i:58114.

6. A. Berthomieu And J.-M. Bismut, Quillen metrics and higher analytic torsion forms, J. Reine Angew. Math. 457 (1994), 85-184, MR 96d:32036.

7. J.-M. Bismut, The Atiyah-Singer theorems: a probabilistic approach. I. The index theorem, J. Funct. Anal. 57(1) (1984), 56-99, MR 86g:58128a.

8. J.-M. Bismut, The Atiyah-Singer index theorem for families of Dirac operators: two heat equation proofs, Invent. Math. 83(1) (1986), 91-151, MR 87g:58117.

9. J.-M. Bismut, Holomorphic families of immersions and higher analytic torsion forms, Astérisque 1997(244). viii+275, MR 2000b:58057.

10. J.-M. Bismut, Duistermaat-Heckman formulas and index theory, in Geometric Aspects of Analysis and Mechanics (ed. Johan A. C. Kolk and Erik P. van den Ban), Progress in Mathematics, Volume 292, pp. 1-55 (Birkhäuser/Springer, New York, 2011), MR 2809 466.

11. J.-M. Bismut, Hypoelliptic Laplacian and orbital integrals, in Annals of Mathematics Studies, Volume 177 (Princeton University Press, Princeton, NJ, 2011), MR 2828080.

12. J.-M. Bismut, Hypoelliptic Laplacian and Bott-Chern cohomology, in Progress in Mathematics, Volume 305 (Birkhäuser/Springer, Cham, 2013). A theorem of Riemann-Roch-Grothendieck in complex geometry, MR 3099098.

13. J.-M. Bismut And D. S. Freed, The analysis of elliptic families. I. Metrics and connections on determinant bundles, Comm. Math. Phys. 106(1) (1986), 159-176, MR 88h:58110a.

14. J.-M. Bismut, H. Gillet And C. Soulé, Analytic torsion and holomorphic determinant bundles. II. Direct images and Bott-Chern forms, Comm. Math. Phys. 115(1) (1988), 79-126, MR 89g:58192b.

15. J.-M. Bismut And S. Goette, Families torsion and Morse functions, Astérisque (275) (2001), x+293, MR 2002h:58059.

16. J.-M. Bismut And S. Goette, Equivariant de Rham torsions, Ann. of Math. (2) 159(1) (2004), 53-216, MR 2051391 (2005f:58059).

17. J.-M. Bismut and G. Lebeau, Complex immersions and Quillen metrics, Publ. Math. Inst. Hautes Études Sci. 1991(74) (1992), ii+298 pp., MR 94a:58205.

18. J.-M. Bismut And J. LotT, Flat vector bundles, direct images and higher real analytic torsion, J. Amer. Math. Soc. 8(2) (1995), 291-363, MR 96g:58202.

19. J.-M. Bismut, X. MA And W. Zhang, Opérateurs de Toeplitz et torsion analytique asymptotique, C. R. Math. Acad. Sci. Paris 349(17-18) (2011), 977-981.

20. J.-M. Bismut And É. VASSERot, The asymptotics of the Ray-Singer analytic torsion associated with high powers of a positive line bundle, Comm. Math. Phys. 125(2) (1989), 355-367, MR 91c:58141. 
21. J.-M. Bismut And É. VAsSerot, The asymptotics of the Ray-Singer analytic torsion of the symmetric powers of a positive vector bundle, Ann. Inst. Fourier (Grenoble) 40(4) (1990), 835-848. 1991, MR 92b:58237.

22. J.-M. Bismut And W. Zhang, An extension of a theorem by Cheeger and Müller, Astérisque (205) (1992), 235. With an appendix by François Laudenbach, MR 93j:58138.

23. M. Bordemann, E. Meinrenken and M. Schlichenmaier, Toeplitz quantization of Kähler manifolds and $\mathrm{gl}(N), N \rightarrow \infty$ limits, Comm. Math. Phys. 165(2) (1994), 281-296, MR 1301849 (96f:58067).

24. A. Borel AND N. Wallach, Continuous Cohomology, Discrete Subgroups, and Representations of Reductive Groups, 2nd ed., Mathematical Surveys and Monographs, Volume 67 (American Mathematical Society, Providence, RI, 2000), MR 1721403 (2000j:22015).

25. L. Boutet de Monvel and V. Guillemin, The spectral theory of Toeplitz operators, in Annals of Mathematics Studies, Volume 99 (Princeton University Press, Princeton, NJ, 1981), MR 620794 (85j:58141).

26. L. Boutet de Monvel and J. Sjöstrand, Sur la singularité des noyaux de Bergman et de Szegö, in Journées: Équations aux Dérivées Partielles de Rennes (1975), Astérisque, No. 34-35, pp. 123-164 (Soc. Math. France, Paris, 1976), MR 0590106 (58 \#28684).

27. J. Chazarain AND A. Piriou, Introduction à la théorie des équations aux dérivées partielles linéaires (Gauthier-Villars, Paris, 1981), MR 82i:35001.

28. J. Cheeger, Analytic torsion and the heat equation, Ann. of Math. (2) 109(2) (1979), 259-322, MR 80j:58065a.

29. J. J. Duistermatit and G. J. Heckman, On the variation in the cohomology of the symplectic form of the reduced phase space, Invent. Math. 69(2) (1982), 259-268, MR 84h:58051a.

30. J. J. Duistermant and G. J. Heckman, Addendum to: 'On the variation in the cohomology of the symplectic form of the reduced phase space', Invent. Math. 72(1) (1983), 153-158, MR 84h:58051b.

31. E. Getzler, A short proof of the local Atiyah-Singer index theorem, Topology 25(1) (1986), 111-117, MR 87h:58207.

32. A. W. KnApp, Representation Theory of Semisimple Groups, Princeton Mathematical Series, Volume 36 (Princeton University Press, Princeton, NJ, 1986). An overview based on examples, MR 855239 (87j:22022).

33. X. MA, Functoriality of real analytic torsion forms, Israel J. Math. 131 (2002), 1-50, MR 2003i:58063.

34. X. MA AND G. MARInescu, Holomorphic Morse Inequalities and Bergman Kernels, Progress in Mathematics, Volume 254 (Birkhäuser, Basel, 2007), MR 2339952 (2008g:32030).

35. X. Ma And G. Marinescu, Toeplitz operators on symplectic manifolds, J. Geom. Anal. 18(2) (2008), 565-611, MR 2393271.

36. X. Ma And G. Marinescu, Berezin-Toeplitz quantization on Kähler manifolds, J. Reine Angew. Math. 662 (2012), 1-56, MR 2876259.

37. X. MA AND W. Zhang, Superconnection and family Bergman kernels, C. R. Math. Acad. Sci. Paris 344(1) (2007), 41-44, MR 2286586 (2007k:32023).

38. S. Marshall and W. Müller, On the torsion in the cohomology of arithmetic hyperbolic 3-manifolds, Duke Math. J. 162(5) (2013), 863-888, MR 3047468.

39. V. Mathai And D. Quillen, Superconnections, Thom classes, and equivariant differential forms, Topology 25(1) (1986), 85-110, MR 87k:58006.

40. H. Moscovici and R. J. Stanton, $R$-torsion and zeta functions for locally symmetric manifolds, Invent. Math. 105(1) (1991), 185-216, MR 92i:58199. 
41. W. MüLler, Analytic torsion and R-torsion of Riemannian manifolds, Adv. Math. 28(3) (1978), 233-305, MR 80j:58065b.

42. W. MüLlER, Analytic torsion and $R$-torsion for unimodular representations, J. Amer. Math. Soc. 6(3) (1993), 721-753, MR 93m:58119.

43. W. MÜLLER, The asymptotics of the Ray-Singer analytic torsion of hyperbolic 3-manifolds, in Metric and Differential Geometry, Progress in Mathematics, Volume 297, pp. 317-352 (Birkhäuser/Springer, Basel, 2012), MR 3220447.

44. D. B. RAY AND I. M. Singer, $R$-torsion and the Laplacian on Riemannian manifolds, Adv. Math. 7 (1971), 145-210, MR 45 \#4447.

45. M. Schlichenmaier, Deformation quantization of compact Kähler manifolds by Berezin-Toeplitz quantization, in Conférence Moshé Flato 1999, Vol. II (Dijon), Math. Phys. Stud., Volume 22, pp. 289-306 (Kluwer Academic Publishers, Dordrecht, 2000), MR 1805922 (2001k:53177).

46. M. E. TAYlor, Pseudodifferential Operators, Princeton Mathematical Series, Volume 34 (Princeton University Press, Princeton, NJ, 1981), MR 82i:35172. 